\title{
Multiphase coexistence with sequence fractionation in random block copolymers
}

\author{
Dissertation \\ zur Erlangung des Doktorgrades \\ der Mathematisch-Naturwissenschaftlichen Fakultäten \\ der Georg-August-Universität zu Göttingen
}

vorgelegt von

Alice von der Heydt

aus Regensburg

Göttingen 2011 


\section{7}

Referentin: Prof. Dr. Annette Zippelius

Korreferent: Prof. Dr. Peter Müller

Tag der mündlichen Prüfung: 23.9.2011 


\section{Contents}

$\begin{array}{ll}\text { Abstract } & 1\end{array}$

\begin{tabular}{lll}
\hline & Introduction & 3
\end{tabular}

1.1 Introduction: Constrained self-assembly . . . . . . . . . . . . . . . . . 3

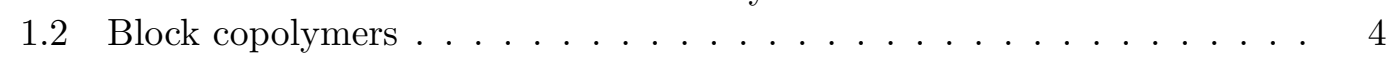

1.3 Random block copolymers . . . . . . . . . . . . . . . . . . . 5

1.4 Experiments . . . . . . . . . . . . . . . . . . . . 6

1.5 Theoretical studies: an overview $\ldots \ldots \ldots \ldots$. . . . . . . . . . 7

1.5.1 Multi-component theory: macroscopic phase separation . . . . . 7

1.5.2 Microstructured phases $\ldots \ldots \ldots \ldots$. . . . . . . . 8

1.5 .3 Influence of fluctuations on the ordered microphase $\ldots . . . .99$

1.5.4 Routes from macroscopic to microscopic phase separation: Phase coexistence? . . . . . . . . . . . . . 10

1.6 Outline of this work $\ldots \ldots \ldots \ldots \ldots \ldots$

$\begin{array}{lll}2 & \text { Model } & 15\end{array}$

2.1 Random $A$ - $B$ sequences $\ldots \ldots \ldots \ldots \ldots$. . . . . . . . . . . . 15

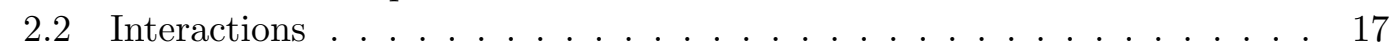

2.2 .1 Polymer chain connectivity . . . . . . . . . . . . . 17

2.2 .2 Excluded volume . . . . . . . . . . . . . . . . . . . . . . 21

2.2 .3 Incompatibility . . . . . . . . . . . . . . . . . . . . 23

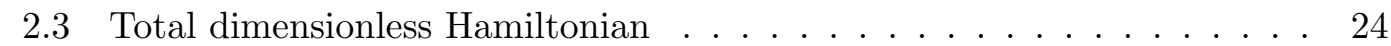

2.3 .1 Note on the number $M$ of segments per block . . . . . . . . . 25

$2.4 \quad$ Field representation of the potentials $\ldots \ldots \ldots . \ldots 26$

\begin{tabular}{|lll}
\hline 3 & Free energy and global equilibrium states & 29
\end{tabular}

3.1 Free-energy functional $\ldots \ldots \ldots \ldots$. . . . . . . . . . . . . . 30

3.1 .1 Partition function . . . . . . . . . . . . . . . 30

3.1 .2 Hubbard-Stratonovich transformation . . . . . . . . . . . . . 31

$3.1 .3 \quad$ Saddle-point approximation and effective Hamiltonian . . . . . . 33

3.1.4 Generalized density field modulations and asymmetric distributions 38

3.2 Instability of the disordered state $\ldots \ldots \ldots 38$

3.3 Global structure function and multicritical point . . . . . . . . . . . . . 40

$3.3 .1 \quad$ Multicriticality for symmetric Markovian sequence distributions. 40

3.3 .2 Multicriticality for triblock copolymers . . . . . . . . . . . . 41

3.3.3 Structure functions for individual triblock sequences . . . . . . . 42

3.4 Lamellar phase separation . . . . . . . . . . . . . . . . . . . . 43

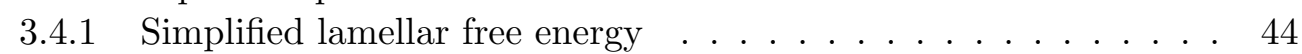

3.4 .2 Free energy with wave-number dependence of fourth order. . . . 45 
iv

$3.5 \quad$ Macroscopic phase separation . . . . . . . . . . . . . . . . 46

3.5 .1 Multiple homogeneous phases . . . . . . . . . . . . . . . 48

3.5 .2 Two symmetric homogeneous phases . . . . . . . . . . . . . . 49

3.5 .3 Two symmetric homogeneous phases in random triblock copolymers 50

\begin{tabular}{|lll}
\hline & Fractionated three-phase coexistence & $\mathbf{5 1}$
\end{tabular}

4.1 General fractionation ansatz . . . . . . . . . . . . . . . . . . . . 52

4.2 Symmetry considerations . . . . . . . . . . . . . . . . . 52

4.3 Constraints at fixed global sequence distribution . . . . . . . . . . . 53

4.4 Three-phase coexistence conditions . . . . . . . . . . . . . 53

4.4 .1 Transition lines to the three-phase region . . . . . . . . . . 55

4.4 .2 Random triblock copolymers $\ldots \ldots \ldots \ldots$. . . . . . . 56

$4.4 .3 \quad$ Solution algorithm $\ldots \ldots \ldots \ldots$. . . . . . . . . . . . . . 57

$\begin{array}{lll}5 & \text { Phase behavior with fractionation } & 59\end{array}$

5.1 Triblocks with a small number of segments per block . . . . . . . . . 59

5.1.1 Scaling of the order-parameter amplitude at the multicritical point 62

5.2 Triblocks with more segments per block . . . . . . . . . . . . . 64

5.2 .1 Scaling of the order-parameter amplitude at the multicritical point 68

5.3 Sequence fractionation for triblocks $\ldots \ldots \ldots \ldots$. . . . . . 69

5.4 Three-phase coexistence for random symmetric diblocks . . . . . . . . 71

5.5 Continuous-chain triblocks analyzed with SCFT. . . . . . . . . . . . 73

$5.5 .1 \quad$ Three-phase coexistence lines . . . . . . . . . . . . . 73

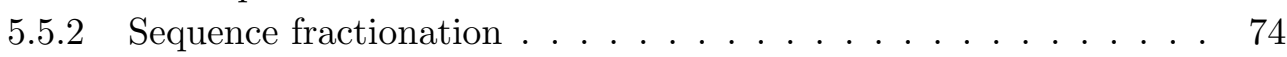

5.5 .3 Discussion $\ldots \ldots \ldots \ldots \ldots \ldots \ldots \ldots$

\begin{tabular}{lll}
\hline 6 & Summary and Discussion & $\mathbf{7 7}$
\end{tabular}

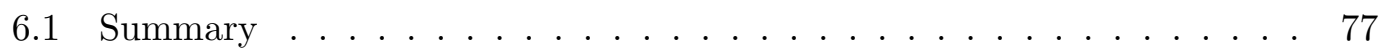

6.2 Discussion . . . . . . . . . . . . . . . . . . . . . . 81

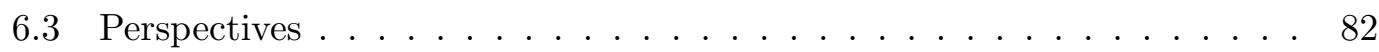

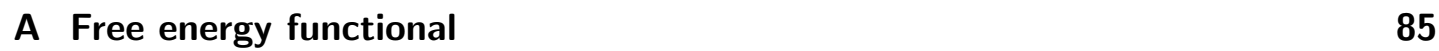

A.1 Hubbard-Stratonovich transformation . . . . . . . . . . . . . . . 85

A.1.1 Saddle points and thermodynamic limit . . . . . . . . . . . 85

A.1.2 $\quad$ Application to our partition function . . . . . . . . . . . . . . . 86

A.2 Quadratic theory for arbitrary sequence distributions . . . . . . . . . . 87

A.2.1 Relation to random phase approximation . . . . . . . . . 87

A.2.2 Response function within RPA $\ldots \ldots \ldots \ldots$. . . . . . 88

A.3 Relation between second-order vertices in $\hat{\sigma}$ and in $\sigma$. . . . . . . . . . . 89

A.3.1 Averages and covariances of $\sigma$ and its conjugate $\hat{\sigma}$. . . . . . 89

A.3.2 Comparison with Fredrickson et al. . . . . . . . . . . . . . . 90

B Composition and sequence distribution of random $Q$-block copolymers 93

B.1 Number and classes of $Q$-block sequences . . . . . . . . . . . . . . 93

B.2 Transition matrix and block-type correlation of Markovian sequences . . 94 
B.3 Moments of composition distribution . . . . . . . . . . . . . . . . . . 94

B.3.1 Second moment . . . . . . . . . . . . . . . . . . . . 96

B.3.2 Fourth moment . . . . . . . . . . . . . . . . . . . . . . . . . . . 97

\begin{tabular}{lr}
\hline C Structure functions & 99
\end{tabular}

C.1 Gaussian-chain conformational averages . . . . . . . . . . . . . . . . 99

C.2 Debye function . . . . . . . . . . . . . . . . . . . 100

C.2.1 Discrete segments . . . . . . . . . . . . . . . . . 100

C.2.2 Continuous-chain limit . . . . . . . . . . . . . . . . . . . . . . . 101

C.3 Second-order structure function . . . . . . . . . . . . . . . . . . . . . . 101

C.4 Three-point and fourth-order structure functions . . . . . . . . . . . . 102

C.5 Structure functions in a symmetric Markovian sequence distribution . . 102

C.5.1 Second-order structure function . . . . . . . . . . . . . . 102

C.5.2 Three-point and fourth-order structure functions . . . . . . . . 103

C.6 Sequence-specific structure functions . . . . . . . . . . . . . . . . 108

C.6.1 $s_{\nu}^{(\alpha)}$ for individual sequences . . . . . . . . . . . . . . . . . 109

C.6.2 $s_{\nu}^{(\beta)}$ for individual sequences . . . . . . . . . . . . . . . . 109

C.6.3 $s_{\nu}^{(\gamma)}$ for individual sequences . . . . . . . . . . . . . 110

\begin{tabular}{|ll}
\hline D Three-phase equilibrium conditions for random triblock copolymers & 111
\end{tabular}

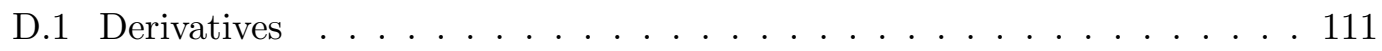

D.2 Constraints . . . . . . . . . . . . . . . . . . . . . 111

\begin{tabular}{ll}
\hline E Macroscopic phase separation & 113
\end{tabular}

E.1 Two and three homogenous phases in random diblocks . . . . . . . . . 113

\begin{tabular}{ll}
\hline List of symbols & 115
\end{tabular}

\begin{tabular}{ll}
\hline Bibliography & 117
\end{tabular}

\begin{tabular}{ll}
\hline Acknowledgments & 127
\end{tabular}

\begin{tabular}{lr}
\hline Curriculum vitae & 129
\end{tabular} 



\section{Abstract}

Random binary block copolymers emerge from linking permanently and at random prepolymer blocks of two different chemical species $A$ and $B$. The competitive interplay of conformational entropy, connectivity within one polymer, temperature-dependent incompatibility between $A$ and $B$, and incompressibility gives rise to a complex phase behavior with a variety of possible morphologies of $A$ - and $B$-rich domains. Technical applications of the self-organized structures in block copolymers include nanoscale templates and medical drug delivery via copolymer micelles.

For random $Q$-block copolymers, this work addresses theoretically the conjectured coexistence of macroscopic phase separation and a structured phase of microscopic $A$ - and $B$-rich domains. Sequence fractionation according to the copolymers' internal structure promotes the coexistence of phases with different morphologies in equilibrium, as is revealed by a theory with explicit account for the exchange of individual sequences. In our semi-microscopic model, one block comprises $M$ identical segments. The Markovian block-type sequence distribution is characterized by the type correlation $\lambda$ of adjacent blocks and the global $A$ content. Our focus is on block copolymer distributions with $A \leftrightharpoons B$ exchange symmetry, for which phase transitions from the disordered state are continuous within mean-field theory. Upon increasing the incompatibility $\chi$ (by decreasing temperature) in the disordered state, we observe the formation of the known global, ordered phases: for $\lambda>\lambda_{c}$, two coexisting macroscopic $A$ - and $B$-rich phases, and for $\lambda<\lambda_{c}$, a microstructured (lamellar) phase with nonzero wave number, $k(\lambda)$. In addition, we encounter a fourth region in the $\lambda$ - $\chi$ plane where these three phases coexist with different, for $Q \geq 3$ non-Markovian, sequence distributions. The three-phase region is reached, either from the macroscopic phases via a third lamellar phase that is rich in alternating sequences, or starting from the lamellar state, via two additional homogeneous, homopolymer-enriched phases; in both cases, the incipient phases have zero volume fraction. The four regions of the phase diagram meet at a multicritical point $\left(\lambda_{c}, \chi_{c}\right)$, at which $A-B$ segregation vanishes. Since our analytical method assumes weak segregation for the lamellar phase, it proves reliable particularly in the vicinity of $\left(\lambda_{c}, \chi_{c}\right)$. For random triblock copolymers, $Q=3$, we find that both the character of this point and the critical exponent of the segregation amplitude change substantially with the number $M$ of segments per block: The lamellar wave number vanishes continuously on approach to $\left(\lambda_{c}, \chi_{c}\right)$ only for $M<7$. The results for $Q=3$ in the continuous-chain limit $M \rightarrow \infty$ are compared to numerical self-consistent field theory (SCFT), which is accurate at larger segregation. 



\section{Introduction}

\subsection{Introduction: Constrained self-assembly}

Segregation phenomena are ubiquitous in everyday situations: oil and vinegar strive to demix in salad dressings, only after considerable stirring do they compromise to form an emulsion. Complete segregation, however, can be hampered by linkage of different
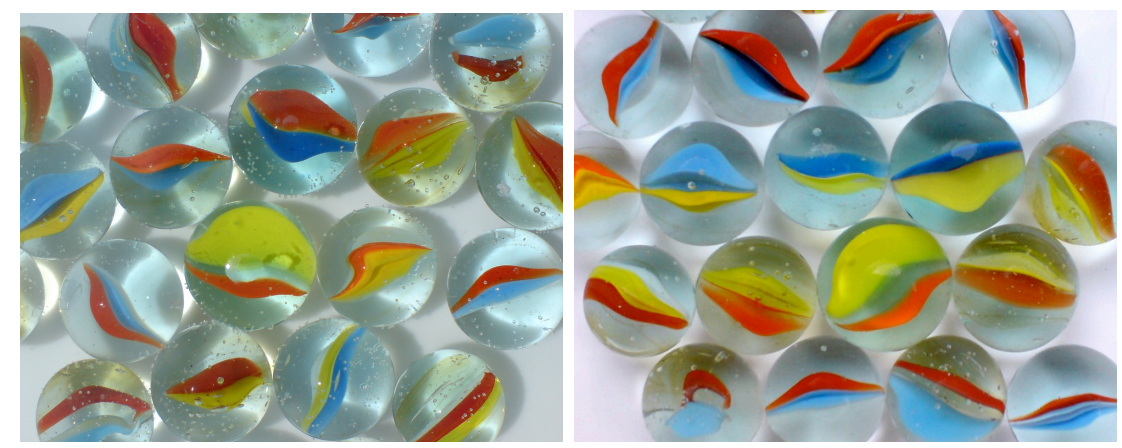

Figure 1.1: Marbles: "disordered" (left) and with colors arranged into "microdomains" (right).

components into one object. Each marble in fig. 1.1, for example, combines multiple colors in its interior. An associated problem you may have experienced when sorting the heap of reprints on your desk is that one article often matches two or more of the named folders you neatly prepared in your register.

Interesting phenomena arise from this kind of frustrated ordering in block copolymers, which are composed of permanently linked prepolymers, called blocks, of chemically different, inmiscible (incompatible) monomer types (cf. fig. 1.2). The effective repulsion between different monomer types increases with increasing Flory incompatibility parameter $\chi$ [37], which is usually inversely proportional to temperature, or with growing degree of polymerization of the blocks. Increasing the incompatibility causes a sponta-

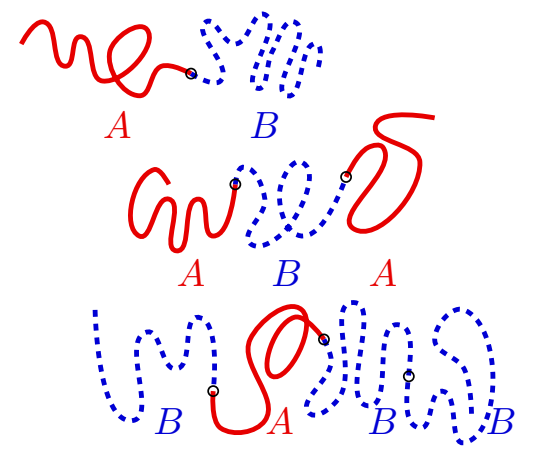

Figure 1.2: Examples of linear, binary $(A B)$ diblock, triblock and quadblock copolymers. 
neous symmetry breaking in an isotropic and homogeneous melt of block copolymers. Macroscopic phase separation is impossible if all copolymers have the same internal sequence of connected, different blocks. Instead, determined by the block length, a periodic pattern of domains enriched in either of the monomer types can be formed, with periods of approximately $10-100 \mathrm{~nm}$ [54], see, e.g., fig. 1.5 below. Due to these domain sizes, which are larger than the average diameter of a single chemical unit, but small relative to the system size, structured phases are often termed microphases; for an overview see, e.g., refs. [11, 12]. The morphology of the domain pattern, e.g. lamellae, spherical micelles, cylinders on a hexagonal lattice, a bicontinuous phase, to name a few, depends on the average composition, the block-length distribution, and the incompatibility, cf. the phase diagram for diblock copolymers in ref. [80].

Both in their mixed state and in their self-organized structures, block copolymers have widespread applications ranging from everyday plastics and adhesives to microelectronics and medical drug delivery by copolymer micelles [12, 93. Block copolymer lithography makes use of the self-assembly of block copolymers, directed by structured surfaces, in order to create specific nanoscale patterns or to improve the order of a given chemically patterned substrate [29, 126, 115]. In genetic engineering, synthesis of protein-based block copolymers with peptide blocks promises precisely tunable structures enabled by the inherent ordering abilities of proteins [96].

\subsection{Block copolymers}

Some properties of block copolymers can be attributed to the parent condensed matter category 'soft matter', where order is attained on a meso- or macroscopic rather than on a molecular scale [71]. In all polymer blends, the rapid decay of the entropy of mixing with increasing degree of polymerization favors phase separation already at moderate incompatibilities [98, particularly macroscopic phase separation in a blend of incompatible homopolymers (polymers composed of one monomer type only). Separation into a periodic structure of microscopic domains, however, is a distinctive feature of block copolymers and stems from the conflict between configurational entropy, connectivity of the inmiscible blocks within one polymer, incompatibility, and in many cases incompressibility. Arranging the connected blocks into the domains costs configurational entropy; therefore, microphase separation requires relatively large blocks compared to homopolymer lengths at which macroscopic phase separation occurs 83 .

The synthesis of block copolymers emerged with the discovery of 'living' polymerization, an anionic, termination-free method initiated by electron transfer to monomers which can be attached sequentially to reactive ends [121]. This technique allows synthesis of, amongst others, monodisperse polymers with equal molecular weight and pure block copolymers of the type $A B A, A B$, and $A B C$, without their respective homopolymers. An example of the first, triblock sequence, is the thermoreversible elastomer poly(styrene)-poly(butadiene)-poly(styrene) (SBS), one representative of the high-impact, rubbery copolymers commercially named Kraton [77]. In order to tailor properties of copolymers, e.g., to achieve toughness and high elasticity combined with bending rigidity as in the example just mentioned, prepolymers with the desired specifications are covalently linked [117, 124, 71]. Some of the tuned properties of a block 


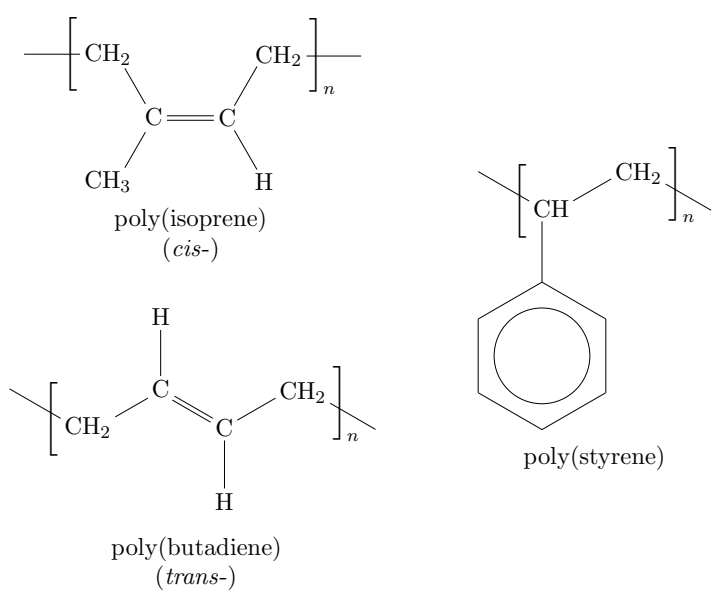

Figure 1.3: Chemical structures of some common block types; see, e.g., 54 .

copolymer in its homogeneously mixed state are bound to be lost in an inhomogeneous state with $A$ - and $B$-rich domains, intriguing new properties may arise. Thus, knowledge about the phase behavior of block copolymers is essential for an advantageous application of their self-assembly.

\subsection{Random block copolymers}

Heterogeneous block copolymers with a random distribution of compositions and block sequences (fig. 1.4 shows possible triblock copolymer sequences) exhibit a greater variety in morphology of the possible phases. The heterogeneity in chemical composition

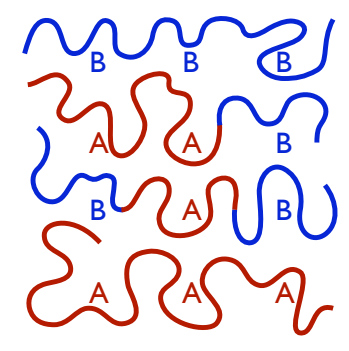

Figure 1.4: Random $A B$ triblock copolymers.

and sequence can arise both from the instantaneous statistical nature of the copolymerization process (instantaneous heterogeneity), and from a steady change in the ratio of concentrations of the different monomer types in the reaction bath (conversion heterogeneity) [114, 113]. Inevitable in some synthesis methods of block copolymers, chemical heterogeneity poses challenges as well as opportunities in technical applications. Given a variance in composition (content of one monomer type) among the copolymers, the system's free-energy optimization cannot only result in several microstructured single-phase morphologies, but also in coexistence of various macroscopic, homogeneous phases with different compositions. From a theoretical point of view, random $A$ - $B$ block copolymer melts are multi-component systems with inherent disorder and competing interactions: entropy and the connectivity of blocks of different types within one copoly- 
mer favor mixing, whereas the incompatibility interaction tends to separate $A$ - from $B$-blocks [11, 42]. While the entropic contributions to the free energy of a copolymer melt dominate at high temperatures, the mainly energetic incompatibility becomes more important at low temperatures. Throughout this work the focus is on melts of random binary $(A-B)$ multiblock copolymers, the chains being linear and monodisperse with respect to their length for the sake of tractability.

\subsection{Experiments}

Transmission electron microscopy (TEM) micrographs gave the first information on patterns in block copolymer systems [56, 122, 23]. With experiments on single-component poly(styrene)-poly(isoprene) (PS-PI) diblocks, each experiment with another composition of the diblocks, Khandpur et al. [72] mapped out a phase diagram that covers various morphologies. Besides the classical ordered microdomain structures (lamellae, spheres on a bcc lattice, hexagonally arranged cylinders), experiments revealed interconnected minimal surface morphologies such as the ordered bicontinuous double diamond (OBDD) [2, 122] and the gyroid phase [53] (the latter structure, hard to distinguish from the former in scattering images, has been confirmed by theory to be a stable equilibrium phase [80]). Extensive studies with small angle X-ray and neutron
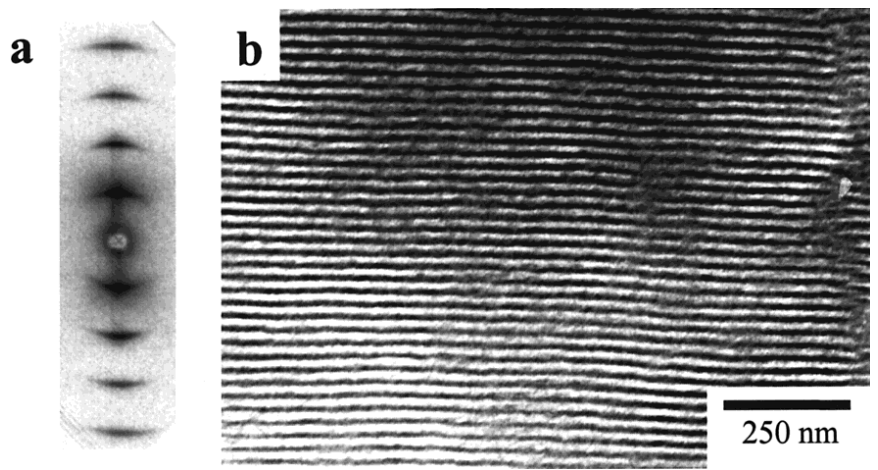

Figure 1.5: Lamellar state in a film of poly(styrene-butadiene-styrene) (SBS); lamellar period $27 \mathrm{~nm}$ : a) small angle X-ray scattering (SAXS) pattern (intensity on logarithmic scale), b) transmission electron microscopy (TEM). Reprinted with permission from [23]. Copyright 2000 American Chemical Society.

scattering (SAXS/SANS) techniques [56, 51, 52], cf. fig. 1.5, provided more insight into the width of the domain interfaces and into the location of single copolymers particularly in microphase-separated states. For example, Hashimoto et al. found that the end blocks of alternating sequences accumulate in the domain interface regions [55]. Another observation concerned the chain conformations in microphase-separated states: block copolymer chains are usually elongated in the direction normal to the interfaces and shortened parallel to the interface [123]. In a series of fractionation and scanning electron microscopy studies, Mirabella et al. [85, 86] characterized the compositional heterogeneity of linear low-density poly-ethylenes (with short-chain branches only) and identified a phase coexistence as responsible for mechanical properties completely 
different from those of the constituents: an extraordinary high fracture toughness arises from the formation of a dispersed soft phase in a hard semi-crystalline matrix.

\subsection{Theoretical studies: an overview}

The first theoretical studies on polymers date back as far as to the 1930s. Kuhn, originally trying to explain the scaling of the viscosity with molecular weight in polymer melts, pioneered the mapping of coarse-grained polymer conformations onto random walks [74. A feature of polymer melts appealing to theorists is that the ideal random walk statistics applies better to the conformations of polymer chains in a dense melt than to those of diluted polymers, cf., e.g., [71, 117]. Therefore, polymer melts will be addressed in the calculations of the present work.

The fact that the entropy of mixing per monomer in solutions of high molecular weight polymers ('high polymers') is drastically reduced, compared to the solution of the same volume fraction of single monomers, motivated Flory's and Huggins's works on the thermodynamics of high polymer solutions [34, 35, 66, 68. Spinodal decomposition of a single-liquid phase due to the low entropy of mixing of high polymers in solution was predicted by Huggins [67]. The resulting, prominent Flory-Huggins theory of demixing in polymer blends (see also [37]) incorporates this entropy reduction in a phenomenological expression for the free energy, motivated by a mean-field, incompressible lattice description similar to the lattice model for a binary liquid. Since the theory assumes uncorrelated placement of monomers onto the lattice, it disregards any other characteristics of polymers, particularly the connectivity. For an incompressible blend of $A$ and $B$ homopolymers, which occupy fractions $v_{A}, v_{B}$ of the system and have degrees of polymerization $N_{A}, N_{B}$, respectively, the proposed free energy per monomer is

$$
\frac{f_{\mathrm{FH}}}{k_{\mathrm{B}} T}=\chi_{\mathrm{FH}} v_{A} v_{B}+\frac{v_{A}}{N_{A}} \ln v_{A}+\frac{v_{B}}{N_{B}} \ln v_{B} .
$$

In this expression, the scalar incompatibility parameter $\chi_{\mathrm{FH}}$ quantifies the interaction between $A$ and $B$ monomers. For the usual case of repulsion between $A$ and $B, \chi_{\mathrm{FH}}>0$, the first term in eq. (1.1) drives $A B$ segregation, whereas the last two terms, resembling the (reduced) entropy of mixing per monomer, favor mixing.

Scott was the first to address phase separation in copolymer mixtures, taking into account the chemical heterogeneity of copolymers [102, 103]. Based on modified FloryHuggins equations for polymer mixtures [36], he calculated the limit of thermodynamic stability (spinodal) of the mixed, single-phase state (see, e.g., [20]) against macroscopic phase separation as a function of the variance in chemical composition (fraction of one segment type in a copolymer).

\subsubsection{Multi-component theory: macroscopic phase separation}

Bauer [13] extended Flory-Huggins theory and Scott's coarse-grained description, determining not only the spinodal of the mixed state, but also the equilibrium compositions of multiple coexisting, homogeneous phases created by phase separation. This method was refined and applied to random $A B$ copolymers by Nesarikar et al. [91, who computed phase diagrams for various average compositions and degrees of polymerization. Within 
this multi-component picture, copolymers are distinguished solely according to their type- $A$ content, while the spatial extension, comprising the particular sequence and the conformation, is disregarded. Increasing incompatibility results in successive transitions to a growing number of macroscopic, homogeneous phases differing in composition. Also based on Flory-Huggins theory, phase coexistence with tricritical points has been analyzed for a mixture of one random $A B$ copolymer with $A$ and $B$ homopolymers [18].

\subsubsection{Microstructured phases}

Taking into account both the internal chain structure and possibly the random distribution of block sequences, however, is crucial for the description of phase separation on microscopic scales [76, 108, 18, 41, 42], e.g., for a lamellar variation of the local density of $A$ blocks, as sketched in fig. 1.6. Identifying this collective density as an

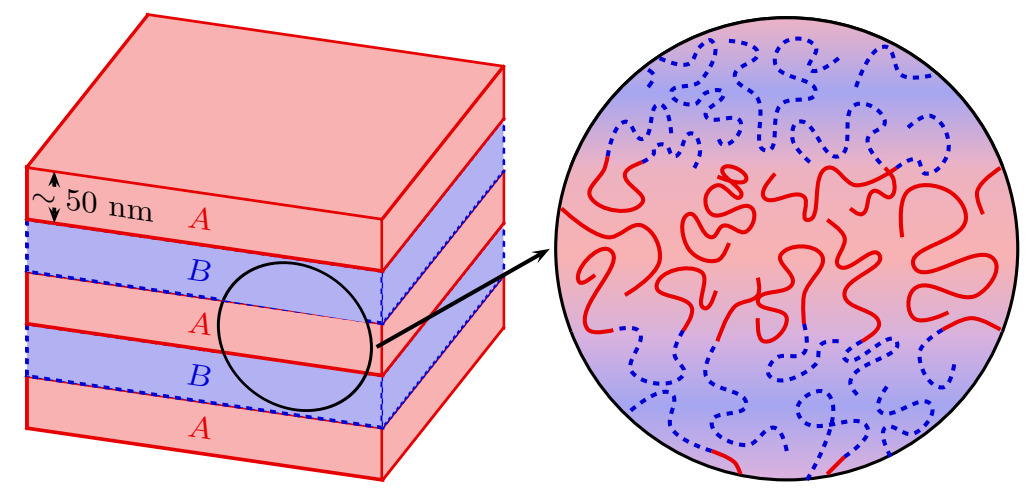

Figure 1.6: Lamellar phase separation. In the blow-up, the self-assembly into $A$ - and $B$-rich domains is exemplified for random triblock copolymers.

order parameter in a Landau approach to phase transitions [75, Fredrickson, Milner, and Leibler [41, 42] started out from a semi-microscopic model to derive the free energy cost associated with spatial composition variations in a random block copolymer melt. They introduced a model of block sequence generation according to a Markov process, resulting in a random distribution with only two parameters to be specified: the global $A$ content, and a correlation parameter $\lambda$ that quantifies the preference for continuing a sequence with a block of the same type as the reactive end. Fredrickson et al. [42] obtained a truncated free-energy functional by treating the model in the limit of $Q \gg 1$ blocks per chain and with some approximations regarding the block-type correlations.

For equal global $A$ and $B$ contents, the phase diagram shows a line of instabilities of the mixed, disordered state, divided into two parts by an isotropic Lifshitz point [61, 60, [22, p. 184] at a critical correlation $\lambda_{c}$ (cf. fig. 1.7). Upon crossing the line by increasing incompatibility, two different ordered (phase) states are possible, depending on $\lambda$. For $\lambda>\lambda_{c}$, a pair of macroscopic, homogeneous phases emerges, one with an excess of $A$, the complement with an excess of $B$ blocks. For $\lambda<\lambda_{c}$, one spatially structured phase with finite wave number, often called microphase, appears. Within mean-field theory, ordered phases emerge from the disordered state via continuous phase transitions in random block copolymers with $A \leftrightharpoons B$ exchange symmetry. 


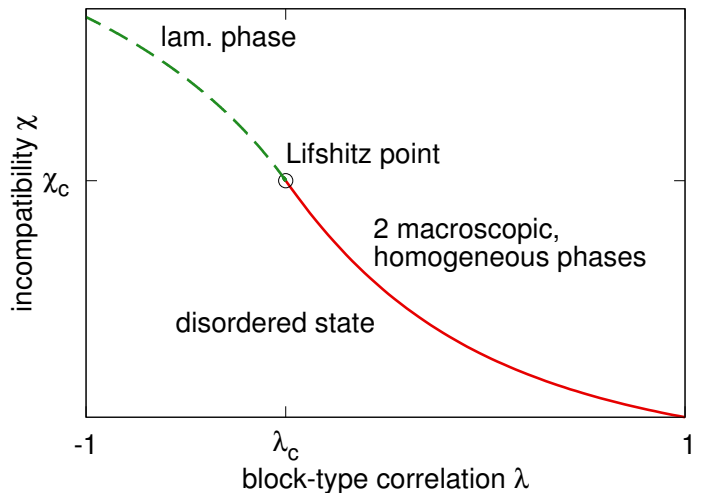

Figure 1.7: Qualitative mean-field phase diagram with the spinodal lines of the disordered state of a Markovian, symmetric block copolymer melt. Microphase separation into an ordered state of $A$ - and $B$-rich domains with finite wave number (here lamellae) occurs to the left of the Lifshitz point.

Although equilibrium states with an ordered structure of $A$ - and $B$-rich domains in random block copolymers are in the focus of our predominantly analytical study, nonequilibrium patterns can obviously arise in copolymers, too. Without claiming completeness, we mention some related studies on the dynamics in the vicinity of a phase transition. For a blend of very long copolymers, each composed of nearly one monomer type only, a glassy state with frozen-in dynamics has been shown to impede the segregation on increasing the incompatibility [15]. In biopolymers such as proteins, freezing may occur due to the restricted number of thermodynamically accessible conformations. Sfatos et al. find that for flexible polymers the frozen state shows a microdomain structure, whereas for stiff polymers freezing takes place first and suppresses microphase formation [106]. Finally, analyzing instead of a random block copolymer melt that undergoes microphase separation the related model system of the constituent, unconnected $A$ and $B$ monomers [27], one finds that macroscopic phase separation is preferred. The transition from the disordered state is discontinuous, however, and Cahn-Hilliard composition waves [21] with period-doubling precede this macroscopic phase separation. Different from the structured, equilibrium phase in the random block copolymer melt (where the basic length scale is set by the size of a block), these waves exist only as a dynamic, transient solution to the set of partial differential equations that describe this kind of phase separation. Yet, the dependence of the wavelength on the distance to the spinodal is comparable to that of the equilibrium state in the copolymer system [27].

\subsubsection{Influence of fluctuations on the ordered microphase}

The nature of the phase transition to an ordered, nonuniform state with finite wave number in block copolymer melts has been a matter of active and controversial discussion. The continuous microphase transition or order-disorder transition (ODT) established in mean-field theory for symmetric block copolymers has been predicted to be affected by order-parameter fluctuations [16, 40].

Some authors first deduced a complete stability of the disordered, homogeneous state against microphase separation [107, 27], but later found that the Landau free energy must contain a local term neglected earlier [108, 42], which restores the microphase separation as a weak first-order transition [50]. Several attempts to improve on the mean- 
field calculations [40, 26, 107, 27] by considering fluctuations of the small-amplitude order parameter employ the weak crystallization theory for the liquid-solid transition developed by Brazovskiı et al. [16, 17]. This theory predicts fluctuation-induced firstorder transitions for isotropic or nearly isotropic systems with an infinite-component order parameter, each component of which is associated with a finite wave number (in the copolymer system, a spatially varying composition field). Since for these systems ordering is degenerate with respect to several directions of the wavevector, fluctuations must be considered not around an isolated point, but in all directions perpendicular to a hypersphere in reciprocal space [120. The Brazovskii model has been applied amongst others to the nematic-smectic $\mathrm{C}$ phase transition in liquid crystals [87], slightly anisotropic antiferromagnets with large-wavelength superstructure [16, and to the Rayleigh-Bénard convective instability in a fluid layer between infinite horizontal plates [119.

Fredrickson and Helfand [40] placed also the effective Hamiltonian of a symmetric, single-component diblock copolymer melt into the class analyzed by Brazovskil and performed a Hartree approximation for this system. Though the free-energy functional does not feature a third-order term, the calculation yields a first-order transition [40, 25] at a higher incompatibility than the continuous microphase transition obtained from the mean-field analysis by Leibler [76]. Apart from the nature of the phase transition, the amplitude and the wave number of the microstructured phase were not altered significantly compared to the mean-field predictions.

Specifically for diblocks, the width of the temperature region dominated by fluctuations has been shown to decay with an inverse power of the degree of polymerization [40, 89]. For the polydisperse system of random block copolymers, a more complicated dependence ensues: fluctuations become less important with increasing block length, but also with decreasing number of blocks per chain [62].

In conclusion, a mean-field description should be adequate for random block copolymers with a small number of blocks addressed in this work, particularly in the case of a large number of segments per block.

\subsubsection{Routes from macroscopic to microscopic phase separation: Phase coexistence?}

Macroscopic phase separation in random block copolymers is found to be restricted to certain ranges of incompatibility $\chi$, block-type correlation $\lambda$, and number $Q$ of blocks per polymer. On the one hand, the mean-field calculations for large $Q$ of Fredrickson et al. 42 showed that at a given correlation $\lambda>\lambda_{c}$, the range of incompatibilities, within which macroscopic, homogeneous phases coexist, shrinks inversely proportional to $Q$. On the other hand, the critical correlation $\lambda_{c}$ (larger values of $\lambda$ increase the preference for homopolymers), above which macroscopic phase separation occurs at all, increases with $Q$ : Upon increasing in a random block copolymer melt the number $Q$ of blocks per polymer and thereby the number of different $A$ - $B$-sequences, the relative variance in $A$ content (composition) decreases as $1 / \sqrt{Q}$. Accordingly, the energy reduction of two $A$-rich and $B$-rich, homogeneous phases relative to the disordered state diminishes, such that the limit $Q \rightarrow \infty$ eventually thwarts macroscopic phase separation, except for a homopolymer blend [104, 118, 62]. 
Monte Carlo simulations for symmetric random block copolymer melts with different $Q$ were performed by Houdayer and Müller [62, 63. They observed macroscopic phase separation for small $Q$ only (deviating from the mean-field results [42]), in a $\lambda$-range shrinking with increasing $Q$.

Additionally, in those simulated systems with macroscopic phase separation, further increase of the incompatibility in the two coexisting homogeneous phases produced a remixed state [63]. This observation lent support to the hypothesis of a coexistence of two homogeneous phases with a third microstructured phase of symmetric composition.

A simulation result for phase coexistence in a binary blend of $A$ homopolymers and $A B A$ triblock copolymers [109] is shown in fig. 1.8 .

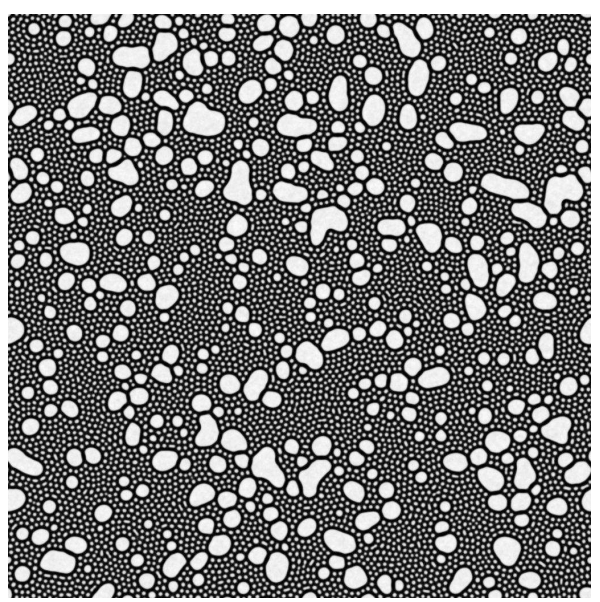

Figure 1.8: $A$-segment density graph of a field-based simulation of a molten blend of $A$-homopolymer with $A B A$-triblocks. Light regions: $A$-rich macroscopic phase, dotted regions: microphase (hexagonally arranged $B$ rich cylinders) of $A B A$. Simulation cell size $256 R_{G} \times 256 R_{G}, R_{G}$ copolymer radius of gyration. Reprinted from [109], Copyright 2003, with permission from Elsevier.

For random diblock copolymers $(Q=2)$, phase coexistence has been predicted by simulation [88], and within mean-field by solutions of the self-consistent field theory (SCFT) equations [70, 90]. The latter calculations, however, obtained contradicting results for the phase states: a coexistence of different, asymmetric lamellae or a one-phase region of so-called nonperiodic lamellae.

Still, as yet, the details of the route from macroscopic to microstructured phase separation, possibly proceeding via a three-phase coexistence, remained largely unclear.

\subsection{Outline of this work}

In an attempt to bridge this gap, this work aims at an analytical picture of threephase coexistence in random block copolymers, enabled by sequence exchange between subsystems with different morphologies, called sequence fractionation.

Starting from a microscopic model that captures the essential interactions, we first derive an effective Hamiltonian in a field coupled to the local difference of $A$ and $B$ segment densities. This field corresponds to the order parameter for $A-B$ separation mentioned above [76, 42].

In order to study spatially structured, ordered phases analytically, we assume small field amplitudes (weak-segregation limit) and consequently expand in the field, resulting in a free-energy functional. Whereas the random sequence model follows Fredrickson and co-workers [42], our derivation of the free-energy functional for structured phases 
follows a different approach and is valid for another system class. Approximations and restricting assumptions made in ref. [42] (partially pointed out in [125]) are avoided, particularly that of a large number $Q$ of blocks per copolymer, which led to dropping free-energy terms subdominant in $Q$. Contrastingly, we specifically address random block copolymers with small $Q$, allowing us to calculate explicitly the contributions of individual sequences to the global structure functions in the free-energy functional. (With this focus, we also have to abstain from a coarse-graining of adjacent blocks' correlations adopted in [125].)

For macroscopic phase separation, a closed free-energy expression can be derived within the framework of multicomponent theory without the need to resort to an expansion in the order-parameter amplitude.

With a superposition of the free energies of lamellae and homogeneous phases, we arrive at an ansatz for a multi-phase coexistence, parameterized by sequence concentrations and volume fractions. Via sequence fractionation, this coexistence of phases with different morphologies can attain a lower free energy than a single global phase: Each sequence class prefers that phase - homogeneous or spatially structured - which matches best its (non)uniform internal structure, characterized amongst other features by the number of bonded $A-B$ contacts. As a result, the homogeneous phases and the coexisting microstructured phase display different equilibrium concentrations of the same sequence class. Our phase diagrams are calculated for global sequence distributions with $A \leftrightharpoons B$ exchange symmetry, which for Markovian sequence distributions is equivalent to equal $A$ and $B$ content. This symmetry causes the sequence distributions of two $A$-rich and $B$-rich, homogeneous phases to map onto each other by permutation of $A$ and $B$. The individual distributions of these two phases, though different in composition, are not called fractionated, since they maintain the global concentration of a sequence class, e.g., of $A A B / B B A$ in random symmetric triblock copolymers. The $A$-rich phase in this system successively substitutes $B B A$ chains with $A A B$ chains, inversely the $B$-rich phase. In contrast, we define sequence fractionation to alter the sequence class concentrations in parts of the system such that microstructured phase separation is favored in one part, while macroscopic phase separation occurs in another part.

The analytical fractionation approach is explored in most detail for the symmetric triblock copolymer system [58, 59]. In the control-parameter plane spanned by block-type correlation $\lambda$ and incompatibility $\chi$ (cf. fig. 1.7), we can delineate a three-phase coexistence region of two homogeneous, $A$ - and $B$-rich phases and one lamellar (micro)phase, flanked by a region of global macroscopic phase separation on one side, and by one of global lamellar phase separation on the other side. An increase of the incompatibility in a macroscopically phase-separated state results in a third, lamellar phase with zero volume fraction (called shadow, following the nomenclature of [110]), with finite amplitude, and, compared to the global sequence distribution, enriched in alternating sequences. Conversely, if the first instability is towards lamellar phase separation, upon increasing incompatibility two additional $A$ - and $B$-rich homogeneous phases appear as shadows with finite amplitude. In effect, four states, viz. the aforementioned, and the disordered, homogeneous state, coincide at a multicritical point.

The character of this point is found to depend subtly on the model block copolymers' internal structure, namely the number of segments per block. For triblock copolymers with a small number $(M<7)$ of discrete segments per block, the wave number of the 
incipient lamellar phase vanishes continuously on approach to the multicritical point (the latter being therefore of the Lifshitz type), and the order-parameter amplitude vanishes linearly. For triblock copolymers with higher numbers of segments per block, particularly including the limit of continuous chains, the wave number of the ordered state attains a finite value discontinuously when reaching the multicritical point along the line of macroscopic phase separations. In the latter case, for correlation parameters at which the free energy's global minimum indicates lamellar phase separation, a macroscopically phase-separated state corresponding to a local minimum of free energy still persists as a metastable state, and vice versa. In these cases, the critical exponent $\psi$ for the order-parameter amplitude is found to be $\psi=0.5$.

Detailed sequence-concentration diagrams of the coexisting phases show the sequence partitioning according to their morphologies. Except at the multicritical point itself, the shadow phases emerge with a finite deviation from the global, $\lambda$-defined sequence distribution.

The theory for the three-phase coexistence is seen to apply also to 'random' symmetric diblock copolymers.

The structure of this work is as follows:

- In chapter 2 we introduce the model system, the interactions, and the field-based description.

- The partition function is set up in chapter 3, and the saddle-point evaluation yields a free-energy functional that allows for the study of equilibrium phases. In section 3.3, we analyze the behavior of the distribution-averaged structure function which determines the wave number of the instability of the disordered state. For Markovian sequence distributions, we define the critical block-type correlation $\lambda_{c}$ for the wave number to change from zero to a finite value and classify the multicritical points for random triblock copolymer melts. Before exploring the possibilities of three-phase coexistence, free energies are derived for the global ordered states in random block copolymers with $A \leftrightharpoons B$ exchange symmetry, viz. lamellae and two homogeneous $A$ - and $B$-rich phases.

- Decomposing the structure functions explicitly into the individual sequences' contributions allows us to formulate and analyze a three-phase coexistence ansatz with sequence-selective exchange, in chapter 4. This free-energy superposition is optimized for the parameters, viz. volume fraction and sequence concentrations, of the coexisting phases, and we compute the phase transition lines between global phase-separated states and three-phase coexistence.

- Our main results, the phase diagrams as a function of block-type correlation $\lambda$ and incompatibility $\chi$, are presented in chapter 5, as well as additional information on the volume fractions, the wave numbers, the sequence distributions of the coexisting states with fractionation, and the scaling at the multicritical point. We briefly discuss results of a numerical self-consistent field theory (SCFT) study for random continuous triblock copolymers, which complements our analytical method [59]. SCFT is not part of this thesis.

- Chap.6 summarizes the work, concluding with some perspectives. 



\section{Model}

This chapter introduces the basic ingredients of the microscopic model underlying the theoretical description. First, we specify the block copolymer system, multidisperse with regard to composition and sequence, and the random distribution resulting from a Markovian growth process. Next, three interactions are identified as the most important contributions to the Hamiltonian: the bonded potential between monomers or segments adjacent on one chain, the excluded volume interaction and the incompatibility between all segments in the system. Finally, we define the collective density field suitable for the detection of inhomogeneous type distribution in the case of $A$-B-segregation.

\subsection{Random $A-B$ sequences}

Our model system is a melt of $N$ linear, random $A B$ block copolymers in a volume $\tilde{V}$. All copolymers have degree of polymerization $L=Q M$, and are divided into $Q$ blocks, each of which comprises $M$ identical segments (either of type $A$ or of type B),

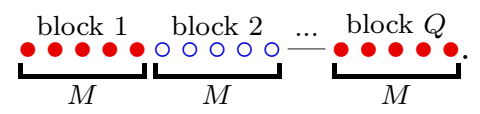

The statistical character and the effective length of these model segments will be explained in the context of the intra-chain bond potential in section 2.2.1. Both types of segments are assumed to have the same molecular weight and statistical length.

In order to formulate the effectively repulsive interaction between segments of different types (see section 2.2.3), we introduce a binary variable $q_{j}(s)$ for the type of segment $s$ on copolymer $j$,

$$
q_{j}(s)= \begin{cases}+1, & s \text { of type } A, \\ -1, & s \text { of type } B\end{cases}
$$

which is equal for segments within the same block $\beta$ (the block number $\beta(s)$ is defined in eq. (B.9) in the appendix). By definition, the mean $q_{j}(s)$ of various blocks, e.g., of one block copolymer, quantifies the $A$ excess of that entity. The average $A$ excess of a block copolymer sequence in a random distribution is denoted as $q$, related to the global $A$ concentration or probability, $p$, via $q=2 p-1$.

A random sequence distribution has to be specified by at least one further parameter for the variance in $A$ content and the degree of blockiness. In our minimal model, block sequences of fixed, finite length are generated by a random copolymerization process with Markovian statistics [42]: the type of a block attached to a reactive end of a growing copolymer is correlated solely with the type of the end block. The vector $\left(p_{A}(\beta), 1-p_{A}(\beta)\right)^{T}$ of probabilities to find $A$, respectively $B$, at block $\beta$ transforms to 
$\left(p_{A}(\beta \pm 1), 1-p_{A}(\beta \pm 1)\right)^{T}$ via the transition matrix $\hat{M}$

$$
\hat{M}=\left(\begin{array}{cc}
1-p_{B A} & p_{A B} \\
p_{B A} & 1-p_{A B}
\end{array}\right),
$$

where $p_{J K}, J, K \in\{A, B\}$, is the conditional probability that a block of type $J$ is attached to one of type $K$. The $p_{\mathrm{JK}}$ are proportional to the corresponding reaction rates $r_{J K}$ and to the concentrations $c_{A}, c_{B}$ of reactive $A$, respectively $B$, blocks in the reaction bath: $p_{\mathrm{JK}} \propto c_{\mathrm{J}} r_{\mathrm{JK}}$. Stationary values shall be guaranteed by steady feeds of both (prepolymer) block types to the reaction bath and rule out a conversion heterogeneity such as described in [113]. Moreover, the rates at which a reactive block of a given type starts a new copolymer or binds to a growing copolymer shall be equal. Thereby, the parameters of the distribution become homogeneous and apply at any position or block number within a copolymer. The first parameter of the sequence distribution is the mentioned, global $A$ probability $p$ and appears in the stationary probability vector associated with the eigenvalue 1 of $\hat{M}$,

$$
p:=\frac{p_{A B}}{p_{A B}+p_{B A}} .
$$

A second parameter drawn from $\hat{M}$ accounts for the sequence polydispersity and measures the type correlation of blocks adjacent on a chain or the average length of subsequences of one type: 1

$$
\lambda:=\left(1-p_{A B}-p_{B A}\right) \in[-1,+1] .
$$

The block-type correlation $\lambda$ proves to be the second eigenvalue of the transition matrix $\hat{M}$ and belongs to the non-stationary part of the process (aside from the limiting cases $\lambda= \pm 1$, the process is ergodic with detailed balance, such that the stationary vector is unique [105]). Positive values of $\lambda$ signal a preference for homopolymers, $\lambda=0$ describes ideal, i.e., uncorrelated random block sequences with a binomial composition distribution, and for negative values of $\lambda$ alternating sequences prevail:

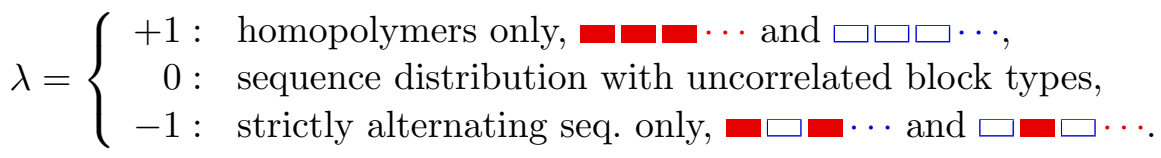

The Markovian synthesis process amounts to creating the simplest nontrivial sequence distribution with two parameters, $p$ and $\lambda$, only. Elements of the transition matrix expressed by $p$ and $\lambda$, for general and for symmetric $(p=1 / 2$ or $q=0)$, Markovian block copolymers, can be found in appendix B.2. A diagonal representation of $\hat{M}$ serves to compute the probabilities of individual sequences in the global $\lambda$-distribution and the moments of $A$ excess in this distribution. Note that Markovian block copolymer distributions with $q=0$ display $A \leftrightharpoons B$ exchange symmetry, which we will use in chap. 3 below. Solely the property $q=0$ of an otherwise arbitrary sequence or sequence

\footnotetext{
${ }^{1}$ Other models introduce instead of $\lambda$ the normalized length variance of subsequences of one type as a parameter for the polydispersity of the block copolymers, see, e.g., 25].
} 
distribution does not imply $A \leftrightharpoons B$ exchange symmetry; consider for example the quadblock sequence $B A A B$.

An individual block copolymer in the distribution can contain 0 to $Q$ blocks of type $A$, which defines $Q+1$ components distinguished by the $A$ content. This component classification in the 'crushed polymer approximation' (see, e.g., [13], 91]) is sufficient to study separation into homogeneous phases, cf. section 3.5 below. However, it neglects differences in the block-type sequence (for example, $A A B$ - and $A B A$-chains both have $A$ content $2 / 3$ ), and usually also in the conformations, details of the internal copolymer structure which are crucial for ordering at finite wavelengths. The number of possible different sequences (without directionality) increases exponentially with the number of blocks, $Q$, and is given by

$$
\mathcal{N}(Q)=2^{Q-1}+2^{\left\lceil\frac{Q}{2}\right\rceil-1}
$$

(see the derivation and the definition of sequence classes in appendix B.1).

Once generated, the block-type sequences remain fixed, i.e., thermal averages affect only the copolymers' conformations and center-of-mass positions. For a finite number of different sequences, a concentration for each sequence is well-defined in the thermodynamic limit. Hence, for finite $Q$, the quenched randomness [19] due to the fixed block types can be effectively translated to a multi-component system of compound particles with internal interactions.

\subsection{Interactions}

\subsubsection{Polymer chain connectivity}

Most of the unusual statistical behavior of polymers in general can be traced back to the linking of many chemically identical repeat units or monomers into a macromolecule. The entropy per monomer related to the positioning of polymers in a given volume is much smaller than that for the mixture of the constituting single monomers ${ }^{2}$ The phenomenological Flory-Huggins theory [65, 34] accounts for this reduced entropy simply with a prefactor of the inverse degree of polymerization, cf. eq. (1.1), but neglects the actual connected structure of polymers: An assumption implicit in the Flory-Huggins mean-field treatment is that monomers of each type can be placed onto the lattice sites without correlations between linked monomers. Here instead, we choose to consider the connectivity via a potential, which imposes further constraints, but which also affords the chains with additional conformational entropy. This potential is categorized as bonded since it acts between nearest neighbors along the chain only. Explicitly, we will focus on linear polymer chains formed by covalent bifunctional bonds. Since the typical energy of a covalent bond $(\sim 5 \mathrm{eV})$ is by orders of magnitudes larger than that of a contact between non-adjacent segments $(\sim 0.1 \mathrm{eV})$ or the thermal energy at moderate temperatures, the linear sequence can be considered as fixed on the time scale of experiments [49]. For the connectivity we will not distinguish between $A$ and $B$ monomers, assuming that the weights for chain configurations of a copolymer are equal

\footnotetext{
${ }^{2}$ To grasp this fact intuitively, start to count the number of ways to arrange a collection of single LEGO ${ }^{\circledR}$ blocks in a cylindrical container. Then, try to fit some pillars build of the same blocks into the container and repeat the counting.
} 
to those of a polymer made up of identical units, i.e., a homopolymer. The motivation to approximate connectivity by an idealized, coarse-grained potential is both mathematical tractability and the expectation that atomistic details of the constituting molecules will not severely influence mesoscopic observables as the average coil size [99, Ch. 2]. In the fluid state, polymers can switch between a large number of conformations whose weights are determined by the Boltzmann statistics. The fact that in the vast number of isomeric states of a polymer, stretched conformations have by far less realizations than coiled ones, is reflected in the Gaussian-chain model [28, 117], whose derivation will be sketched here.

Assuming a given length $\ell$ for each bond along a polymer backbone, an individual steric conformation can be described by the angles between consecutive bonds. These are the bond angle $\vartheta$ between two adjacent bonds and the torsion angle $\varphi$ formed by a bond with the plane of its two preceding bonds (cf. fig. 2.1). Usually, the angles take some preferred values depending on the potentials for bending and torsion. For carbon-carbon bonds in polyethylene, for instance, the bond rotation potential has three minima with respect to the torsion angle $\varphi$, corresponding to staggered relative positions or maximal separation of the $\mathrm{H}$ atoms of the methyl groups [38, Ch. 2], [82]. However, these bond angles will be subjected to fluctuations, and the angle correlation

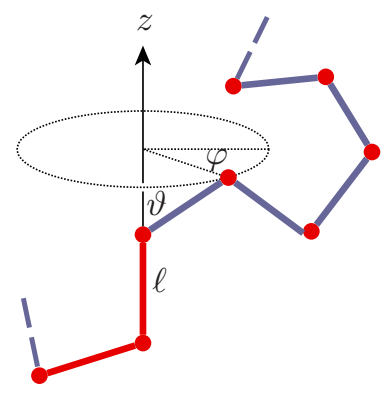

Figure 2.1: Bond angles and loss of directional correlations with chemical distance.

between a given chain section (parallel to $z$ in fig. 2.1) and another section will die off with increasing number of in-between monomers, i.e., with chemical distance. The associated degree of flexibility of a polymer can be expressed via a length scale: On an intermediate level of resolution $(\sim \mathrm{nm})$, the polymer chain appears as a smooth curve, along which one can introduce an arc-length parameter $l \in\left[0, l_{c}\right], l_{c}$ the total contour length, and a tangential vector field $\boldsymbol{n}(l)$ which indicates the local chain direction. A normalized function $K_{\text {or }}(\Delta l)$ measures the directional correlation depending on the distance $\Delta l$ along the chain,

$$
K_{\mathrm{or}}(\Delta l)=\langle\boldsymbol{n}(l) \cdot \boldsymbol{n}(l+\Delta l)\rangle_{\mathrm{or}},
$$

and decays to zero for $\Delta l \rightarrow \infty$. This correlation in turn defines the persistence length

$$
l_{p}:=\int_{0}^{\infty} \mathrm{d} \Delta l K_{\mathrm{or}}(\Delta l),
$$

a typical chain section over which the direction is memorized. Now, resolving the chain only down to a minimal length scale larger than $l_{p}$, the following reduction of the configurational variables is suitable in order to obtain statistical information on the 


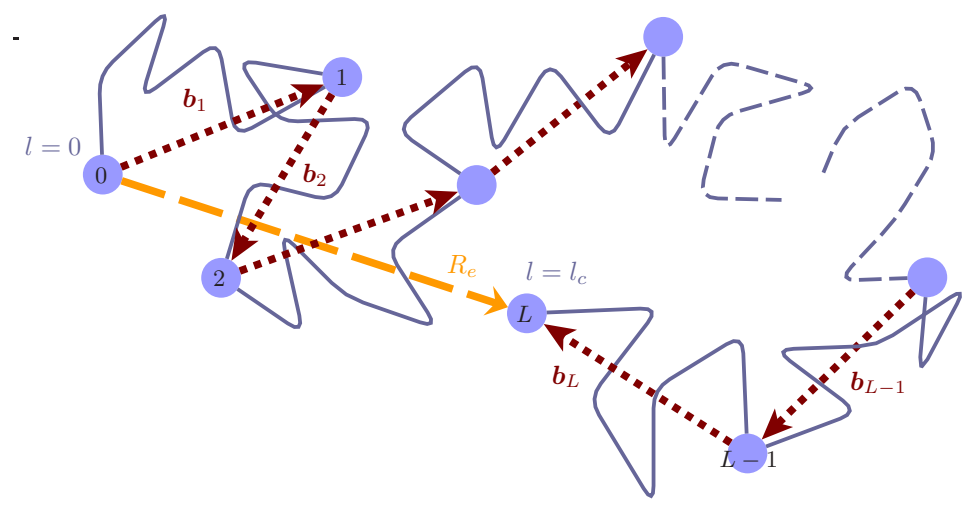

Figure 2.2: Construction of an ideal-chain representation with effective segments $\boldsymbol{b}_{s}$ replacing equal-sized portions of the original polymer with total contour length $l_{c}$. The end-to-end vector $\boldsymbol{R}_{e}$ is indicated by a dashed arrow.

polymer shape, such as, e.g., the distribution of the end-to-end vector: Linear segments (vectors $\boldsymbol{b}_{s}, s=1, \ldots, L$, in fig. 2.2 each replace a chain portion with an arc-length longer than $l_{p}$ and a corresponding number of chemical units, such that the directions of subsequent segment vectors are uncorrelated $3^{3}$ The end-to-end vector is

$$
\boldsymbol{R}_{e}=\sum_{s=1}^{L} \boldsymbol{b}_{s}
$$

and the independent angle degrees of freedom are concentrated at the junction points with coordinates $\boldsymbol{R}_{s}, s=0,1, \ldots, L$. With equal average sizes $\left\langle\boldsymbol{b}_{s} \cdot \boldsymbol{b}_{s}\right\rangle=b^{2}$ of the segments $(\langle\cdot\rangle$ the average over all chain conformations), the mean squared end-to-end distance of this freely jointed segment chain is

$$
\left\langle R_{e}^{2}\right\rangle:=\left\langle\boldsymbol{R}_{e} \cdot \boldsymbol{R}_{e}\right\rangle=\sum_{s, s^{\prime}=1}^{L}\left\langle\boldsymbol{b}_{s} \cdot \boldsymbol{b}_{s^{\prime}}\right\rangle=\sum_{s, s^{\prime}=1}^{L}\left\langle\boldsymbol{b}_{s} \cdot \boldsymbol{b}_{s^{\prime}}\right\rangle \delta_{s, s^{\prime}}=L b^{2} .
$$

and must coincide with the average squared diameter of the real coil. By construction, this chain representation resembles a random walk [74], with the well-known probability distribution particularly for the end-to-end vector $\boldsymbol{R}_{e}$ (cf. fig. 2.2 ). For a chain consisting of a large number of such independently, identically distributed segment vectors, the central limit theorem states that the probability distribution of the resulting end-to-end vector is Gaussian,

$$
\mathbb{P}\left(\boldsymbol{R}_{e}\right)=\left(\frac{d}{2 \pi\left\langle R_{e}^{2}\right\rangle}\right)^{d / 2} \exp \left\{-\frac{d R_{e}^{2}}{2\left\langle R_{e}^{2}\right\rangle}\right\}
$$

with $d$ the spatial dimension. Even for a few segments, the exact distribution can be shown to be approximated extremely well by a Gaussian [117, Ch. 2], Therefore, the random-walk statistics can be assumed to hold also for the vectors $\boldsymbol{R}_{s s^{\prime}}$ connecting any two (non-adjacent) junction points $s, s^{\prime}$ :

$$
\left\langle R_{s s^{\prime}}^{2}\right\rangle=\left|s-s^{\prime}\right| b^{2} .
$$

\footnotetext{
${ }^{3}$ The additional constraint that segments may not overlap will be addressed in the next section.
} 
This implies self-similarity of the Gaussian chain, hence the possibility to rescale eq. 2.10 with a new number $L_{r}$ and a new average size $b_{r}$ of the model segments according to $L_{r} b_{r}^{2}=L b^{2}$. Consequently, the decrease of the average segment density $\varrho_{p}$ inside the polymer's typical volume with increasing degree of polymerization is approximately described by the fractal relation

$$
\varrho_{p} \approx \frac{L}{R_{e}^{3}} \propto L^{-1 / 2} .
$$

The universal dependence eq. 2.10) of the mean squared end-to-end distance on the segment number itself does not fix the length $b$. This ambiguity can be removed if the dimensions of a polymer both in the maximally stretched and in the coiled state are accessible by measurement. Then, the so-called Kuhn length $b$ [117] is determined in accordance with the following criteria:

- Number $L$ and length $b$ of the model segments are chosen such that the ideal chain law $R_{e}^{2}=L b^{2}$ gives the observed squared end-to-end distance $\left\langle R^{2}\right\rangle=R_{e}^{2}$. Instead of the end-to-end distance, scattering experiments often measure the radius of gyration, which is the r.m.s. distance either of all monomers from the center of mass or between all pairs of monomers (if all monomers have the same mass),

$$
R_{g}^{2}=\frac{1}{L} \sum_{s=0}^{L}\left\langle\left(\boldsymbol{R}_{s}-\frac{1}{L} \sum_{s^{\prime}=0}^{L} \boldsymbol{R}_{s^{\prime}}\right)^{2}\right\rangle=\frac{1}{2 L^{2}} \sum_{s, s^{\prime}=0}^{L}\left\langle\left(\boldsymbol{R}_{s}-\boldsymbol{R}_{s^{\prime}}\right)^{2}\right\rangle .
$$

For an ideal chain, using eqs. 2.10 and 2.12 , one finds $R_{g}^{2}=\frac{L b^{2}}{6}\left(1+\frac{3}{L}+\frac{2}{L^{2}}\right)$, thus $R_{g}$ is related to the end-to-end distance via $R_{g}^{2}=R_{e}^{2} / 6$ to leading order in $L$.

- The added average lengths of the segments reproduce the maximal chain length (to be determined experimentally), called the total contour length $l_{c}, L b=l_{c}$, which combined with the first condition $L b^{2}=R_{e}^{2}$ gives $b=R_{e}^{2} / l_{c}$.

- The Kuhn length $b$ must be larger than the persistence length $l_{p}$. Assuming for the directional correlation eq. (2.7) an exponential with decay constant $l_{p}$ and in the limit $l_{p} \ll l_{c}$, the Kuhn length fulfills $b=2 l_{p}$ [117, Ch. 2].

Lower and upper length bounds for the scaling eqs. 2.12) and (2.13) are, respectively, the persistence length $l_{p}$ and the contour length $l_{c}$ of the real polymer, i.e., $l_{p}<b<R_{e}<l_{c} 4^{4}$

In terms of the model segments $\boldsymbol{b}_{s}:=\boldsymbol{R}_{s}-\boldsymbol{R}_{s-1}, s=1, \ldots, L$, the single-chain connectivity potential that yields the Boltzmann weight eq. (2.11) is

$$
\mathcal{H}_{\mathrm{C}, \text { s.c. }}=\mathrm{k}_{\mathrm{B}} T \frac{d}{2 b^{2}} \sum_{s=1}^{L} \boldsymbol{b}_{s} \cdot \boldsymbol{b}_{s}=\mathrm{k}_{\mathrm{B}} T \frac{d}{2 b^{2}} \sum_{s=1}^{L}\left(\boldsymbol{R}_{s}-\boldsymbol{R}_{s-1}\right)^{2},
$$

i.e., a chain of harmonic springs with constants $d \mathrm{k}_{\mathrm{B}} T / b^{2}$. This connectivity potential is based on the number of available configurations of a polymer, therefore entropic in nature and proportional to $\mathrm{k}_{\mathrm{B}} T$.

${ }^{4}$ Some studies, including more recent ones [1], question the ability of the Kuhn length to correctly describe the configurational statistics especially in polymer dynamics and small-scale structures. 
The ideal-chain scaling can be formally extended to infinitesimally small length scales in the limit

$$
L \rightarrow \infty, b \rightarrow 0 \text { with } L b^{2}=\text { const. }=R_{e}^{2}
$$

referred to as the continuous Gaussian or Brownian chain (with finite coil diameter). This limit process results in a rather artificial object with $l_{c}=R_{e}^{2} / b \rightarrow \infty$, according to the above mentioned procedure. However, the continuous-chain model proves to describe well the properties of certain real polymers, in a range of resolutions between their persistence length and real contour length $\tilde{l}$. Determined by one single parameter, viz. $R_{e}$, the continuous-chain version is suited for numerical treatment of polymer models, see e.g. [39]. Therefore, including the limit of continuous polymer blocks later will allow us to compare with numerical results for the phase diagrams of random copolymer melts. For a continuous Gaussian chain with dimensionless arclength parameter $s \in[0,1]$, the connectivity becomes

$$
\mathcal{H}_{\mathrm{C}, \text { s.c. }}=\mathrm{k}_{\mathrm{B}} T \frac{d}{2 R_{e}^{2}} \int_{0}^{1} \mathrm{~d} s\left|\frac{\mathrm{d} \boldsymbol{R}(s)}{\mathrm{d} s}\right|^{2} \text {. }
$$

Here and in the following, we write $\boldsymbol{R}(s)$ instead of $\boldsymbol{R}_{s}$ for the dependence on the arc-length parameter, respectively the segment index.

\subsubsection{Excluded volume}

The ideal Gaussian (or 'phantom') chain model described in the last section tacitly ignores the finite volume occupied by one segment, i.e., the constraint that segments may not overlap. In general, this constraint induces also intra-chain correlations between segments distant along the chain, but frequently close in space, which repel each other at small distances. For polymer solutions, the global result for the chain dimension is found to be a swelling as compared to the ideal-chain scaling eq. 2.10), for example

$$
R_{e}^{2} \propto L^{6 / 5}
$$

according to Flory's mean-field treatment for polymers in a good solvent and to Edwards' self-consistent field approach [37, 30]. Determination or estimation of the effective intermolecular pair potential $U$ for a given polymer (solution), necessary to assess the excluded volume parameter $v_{e}$, for spherical monomers

$$
v_{e}:=\int \mathrm{d}^{d} R\left(1-\mathrm{e}^{-U(R) /\left(\mathrm{k}_{\mathrm{B}} T\right)}\right)
$$

is a theory in itself which may be appreciated in standard polymer textbooks [28, 98] as well as in more specialized treatises [99.

Remarkably, the excluded volume effect is less pronounced in dense polymer melts (the systems we focus on) than in most polymer solutions [28, 117], a fact which may become plausible given the following argument, cf., e.g., ref. [28]: The excluded volume interaction penalizes contacts between any two segments and hence is roughly proportional to $c^{2}$, with $c$ the segment concentration. The corresponding repulsive forces are then proportional to $c \cdot \nabla c$ and are exerted not only between segments on the same 


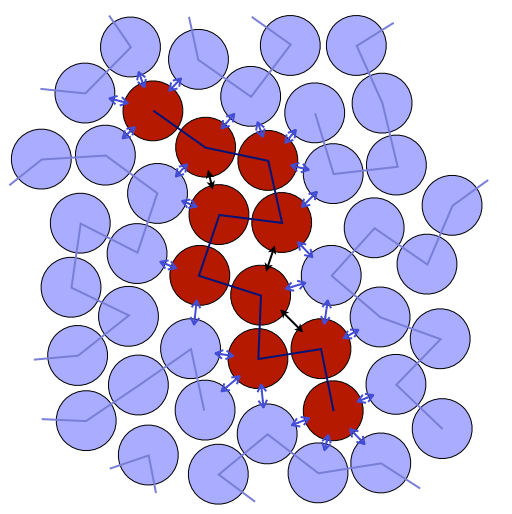

Figure 2.3: Repulsive forces acting on a polymer segment in a dense melt due to both non-adjacent segment on its own chain and segments on foreign chains.

chain, but also between segments residing on different chains. At high concentrations, the chains in the melt are entangled, the segment concentration $c$ becomes almost uniform, and thus the gradients $|\nabla c|$ small. Because of the strong interdigitation, a single segment is on average surrounded by equal amounts of segments of its own chain and of foreign chains (except perhaps for the terminal segment; see fig. (2.3)). Intra-chain repulsion acts in favor of chain swelling, opposed by the forces experienced by segments of other chains, such that the intra- and interchain parts of the excluded volume force approximately cancel. Without further interactions, polymers in a dense melt thus display the ideal-chain behavior, a phenomenon first explained by Flory [37, ch. 12], theoretically founded by Edwards [31], and corroborated by neutron scattering [73]. (Effective depletion of the excluded volume forces may occur also in polymer solutions with a poor solvent at the $\theta$-temperature, where the swelling is hindered by the polymer-solvent repulsion [38].)

To summarize, our restriction to copolymer melts allows us to keep the ideal-chain conformational statistics as a reasonable approximation. However, due to the form of the incompatibility interaction which effectively creates an attraction between segments of the same type (cf. sec. 2.2.3 below), we have to introduce a short-ranged repulsion between any two segments irrespective of their type. In the following calculations, it will turn out essential to start with a finite compressibility, in order to later impose incompressibility, a condition approximately realized in many dense polymer melts [98]. For our purposes, it suffices to model the excluded volume interaction as

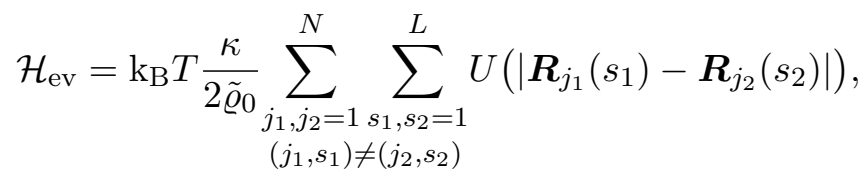

with $\tilde{\varrho}_{0}$ the average segment density, $\kappa$ a nonnegative strength parameter and $U(R)$ a nonnegative, short-ranged and normalized shape function with units of a density. The subscript $j$ of a segment position vector $\boldsymbol{R}_{j}(s)$ indexes the chain, the argument $s$ the segment number within the chain. Since segments that occupy the same site reduce translational entropy, the excluded volume repulsion has a chiefly entropic origin, such as the connectivity, reflected in the prefactor of $\mathrm{k}_{\mathrm{B}} T$ in eq. 2.19 . 


\subsubsection{Incompatibility}

The interactions of polymer chains with identical repeat units considered so far can be completed by one further potential in order to model co- instead of homopolymer chains, provided that prepolymers $A$ and $B$ do not differ in their Kuhn lengths (a non-vital assumption to keep the model tractable) and, more importantly, provided that single-chain conformations remain mostly unaltered on (de)mixing $A$ and $B$.

On intermediate length scales, uncharged molecules attract each other via van der Waals interactions. If the attraction between segments of different types is smaller than between equal ones, which is more frequent than the opposite behavior [98, Ch. 4.2], there is a net repulsion between $A$ and $B$. This effective repulsion is experienced for example by a segment of type $A$, which is translated from a surrounding of pure $A$ to an average surrounding of equal amounts of $A$ and $B$ segments. By expressing contact probabilities of all pairs of segment types in terms of global $A$ and $B$ concentrations, the energy change on mixing $A$ and $B$ can be estimated in a mean-field fashion [117, Ch. 4.1],[98, Ch. 4.2]. Though this conventional definition applies strictly only to the original lattice model by Flory and Huggins [37], we shall allude to it as a motivation for the strength parameter of our incompatibility interaction: The incompatibility in a binary system is defined as a local, scalar quantity via the difference in contact energies (see fig. 2.4)

$$
\chi:=\frac{\bar{z}}{2} \frac{2 u_{A B}-u_{A A}-u_{B B}}{\mathrm{k}_{\mathrm{B}} T},
$$

where $\bar{z}$ is the coordination number of a monomer, and, for continuous positions, corresponds to the average number of nearest neighbors.

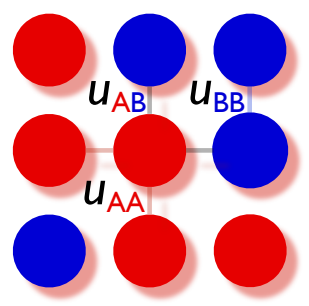

Figure 2.4: Mean-field surrounding of a segment in a binary copolymer melt (lattice version) and pair potentials that define the incompatibility.

Defined as an energy ratio per lattice site, $\chi$ depends on the lattice cell volume, and thus in a continuous-space description must be supplemented by specifying the average monomer density $\tilde{\varrho}_{0}$. With contributions from the pair interaction potentials, but also based on an averaging process over the surrounding of a segment, $\chi$ contains an enthalpic (E) and an entropic (F) part, and, according to the mean-field definition, is of the form $\chi=\frac{\chi_{\mathrm{E}}}{T}+\chi_{\mathrm{F}}$. Since usually $\chi_{\mathrm{F}} \ll \chi_{\mathrm{E}}$, the main contribution to $\chi$ is enthalpic, such that approximately, $\chi$ is inversely proportional to temperature, and increasing incompatibility is equivalent to cooling. Values of the dimensionless incompatibility parameter for various polymer blends can be found in [8, Ch. 19]. A more detailed analysis of the thermodynamics of polymer blends, especially of the correlations in the liquid, relating them to microscopic features using the Ornstein-Zernike integral equation, can be found in refs. [100, 101]. These studies reveal, supported by results from scattering experiments, that in general, the effective $\chi$ can be a nonlocal, wavenumber-dependent quantity due to long-ranged correlations and can depend in a more complicated manner 
on temperature and average density. Helfand [57] argued that the thermodynamical meaning of the dimensionless parameter $\chi$ was unclear and proposed a different, but less common definition of incompatibility. For our present minimal, mesoscopic model, however, in which separate potentials account for polymer connectivity and excluded volume, we can resort to a scalar strength parameter $\chi$ with the motivation introduced above. Accordingly, we formulate the incompatibility potential as

$$
\mathcal{H}_{\chi}=-\mathrm{k}_{\mathrm{B}} T \frac{\chi}{2 \tilde{\varrho}_{0}} \sum_{\substack{j_{1}, j_{2}=1 \\\left(j_{1}, s_{1}\right) \neq\left(j_{2}, s_{2}\right)}}^{N} \sum_{s_{1}, s_{2}=1}^{L}\left(q_{j_{1}}\left(s_{1}\right)-q\right)\left(q_{j_{2}}\left(s_{2}\right)-q\right) W\left(\left|\boldsymbol{R}_{j_{1}}\left(s_{1}\right)-\boldsymbol{R}_{j_{2}}\left(s_{2}\right)\right|\right),
$$

with a nonnegative, normalized, and short-ranged shape function $W(R)$. The type variables $q_{j}(s)$ (see eq. (2.2)) and a positive value of $\chi$ guarantee that contacts of different segment types are unfavorable, whereas segments of equal types attract each other. Subtracting $q$ from each type variable merely adjusts the zero of the incompatibility potential for the case of a global excess of one type.

\subsection{Total dimensionless Hamiltonian}

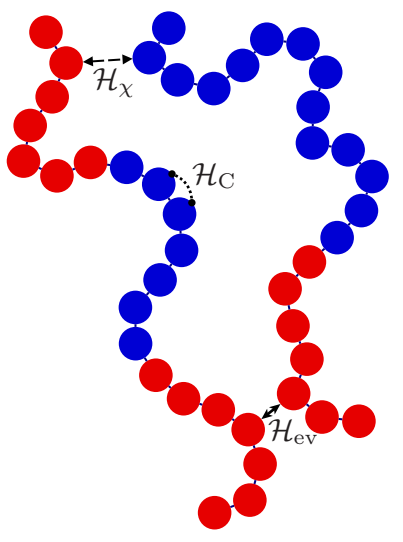

Figure 2.5: Parts of the total Hamiltonian: $A$ - $B$ incompatibility $\mathcal{H}_{\chi}$, polymer connectivity $\mathcal{H}_{\mathrm{C}}$, excluded volume interaction $\mathcal{H}_{\mathrm{ev}}$.

After enumerating the basic intra- and interchain interactions on a mesoscopic level, viz. the harmonic connectivity of segments within an ideal Gaussian chain, the excluded volume and the incompatibility interaction between all segments in the system (cf. fig. 2.5, we can assemble the total Hamiltonian $\mathcal{H}$,

$$
\mathcal{H}:=\mathcal{H}_{\mathrm{C}}+\mathcal{H}_{\mathrm{ev}}+\mathcal{H}_{\chi} .
$$

For convenience, and for consistency with standard notation, we rescale spatial coordinates $R_{\alpha}$ with the Kuhn segment length $b$ and the spatial dimension $d$ as

$$
r_{\alpha}:=\frac{\sqrt{2 d}}{b} R_{\alpha}, \quad \alpha=1,2, \ldots, d .
$$

In effect, we obtain dimensionless monomer positions $\boldsymbol{r}_{j}(s)$, segment vectors $\boldsymbol{b}_{j}(s):=$ $\boldsymbol{r}_{j}(s)-\boldsymbol{r}_{j}(s-1)$, and a dimensionless system volume $V$. The corresponding dimensionless 
average segment density, in the incompressible case constant throughout the system, is

$$
\varrho_{0}:=\frac{N L}{V}=\left(\frac{b}{\sqrt{2 d}}\right)^{d} \tilde{\varrho}_{0} .
$$

The potential shape functions $U$ and $W$ are rescaled accordingly as

$$
\left(\frac{b}{\sqrt{2 d}}\right)^{d} U(R)=: U(r), \quad\left(\frac{b}{\sqrt{2 d}}\right)^{d} W(R)=: W(r)
$$

(keeping, for simplicity, the symbols $U$ and $W$ ), and the dimensionless strength parameters $\kappa$ and $\chi$ are multiplied with a factor two just for the sake of uniform representation. In terms of the rescaled quantities and in units of $\mathrm{k}_{\mathrm{B}} T$, the three parts of $\mathcal{H}$ are

$$
\begin{aligned}
\mathcal{H}_{\mathrm{C}} & =\frac{1}{4} \sum_{j=1}^{N} \sum_{s=1}^{L}\left(\boldsymbol{r}_{j}(s)-\boldsymbol{r}_{j}(s-1)\right)^{2}=\frac{1}{4} \sum_{j=1}^{N} \sum_{s=1}^{L} \boldsymbol{b}_{j}(s) \cdot \boldsymbol{b}_{j}(s), \\
\mathcal{H}_{\mathrm{ev}} & =\frac{\kappa}{4 \varrho_{0}} \sum_{j_{1}, j_{2}=1}^{N} \sum_{s_{1}, s_{2}=1}^{L} U\left(\left|\boldsymbol{r}_{j_{1}}\left(s_{1}\right)-\boldsymbol{r}_{j_{2}}\left(s_{2}\right)\right|\right), \\
\mathcal{H}_{\chi} & =-\frac{\chi}{4 \varrho_{0}} \sum_{j_{1}, j_{2}} \sum_{s_{1}, s_{2}}\left(q_{j_{1}}\left(s_{1}\right)-q\right)\left(q_{j_{2}}\left(s_{2}\right)-q\right) W\left(\left|\boldsymbol{r}_{j_{1}}\left(s_{1}\right)-\boldsymbol{r}_{j_{2}}\left(s_{2}\right)\right|\right) .
\end{aligned}
$$

Anticipating the thermodynamic limit $N \rightarrow \infty$, we have already omitted the restrictions on the sums in the excluded volume and incompatibility interaction eqs. (2.19), (2.21), which subtracted each segment's self-contribution.

Recall that the Gaussian chain connectivity and excluded volume interactions conceptually are effective potentials, which are obtained after integrating-out microscopic degrees of freedom. Due to their chiefly entropic origin, these parts of the Hamiltonian are originally proportional to $\mathrm{k}_{\mathrm{B}} T$, cf. eqs. (2.15) and (2.19). In contrast, the incompatibility is assumed to be dominantly energetic and therefore, in the normalized eq. (2.26c), appears with a temperature-dependent strength parameter $\chi \propto T^{-1}$. In the following, the energy scale is chosen such that $\mathrm{k}_{\mathrm{B}} T$ is unity.

\subsubsection{Note on the number $M$ of segments per block}

As to satisfy statistical independence of adjacent model segment vectors, one segment in the idealized Gaussian-chain connectivity (cf. section 2.2.1) usually represents a large number of chemical repeat units. One interpretation of $M$ is, of course, the length or degree of polymerization of one block. Another picture arises, if we restrict ourselves to blocks with a constant r.m.s. end-to-end distance (which must be much larger than the persistence length $l_{p}$ ): a small number $M$ of segments per block, i.e., a large number of chemical units per segment, indicates that orientational correlations decay slowly along the polymer's contour. Blocks with larger $M$, in the limit $M \rightarrow \infty$ continuous blocks, resemble a fast decay of the orientational correlations. Hence, within the Gaussian-chain approximation for the connectivity, $M$ can also be interpreted as a measure of flexibility of the polymeric filament. There are models for intra-chain potentials tailored specifically to incorporate bending stiffness of a polymer, e.g., the 
wormlike- or persistent-chain model [98, 39, 117]. Nonetheless, it seems worthwhile to first analyze block copolymers with Gaussian-chain connectivity and to study the influence of the parameter $M$ on the phase behavior.

\subsection{Field representation of the potentials}

Preparing for the calculations in the next chapter, we aim at a field-based representation of our model Hamiltonian. In the non-bonded interactions, we can transform from the set of all segment positions $\left\{\boldsymbol{r}_{j}(s)\right\}$ and types $\left\{q_{j}(s)\right\}$ to two density fields:

- The excluded volume interaction, eq. 2.26b can be rewritten as

$$
\begin{aligned}
\mathcal{H}_{\mathrm{ev}} & =\frac{\kappa}{4 \varrho_{0}} \int \mathrm{d}^{d} r \int \mathrm{d}^{d} r^{\prime} \sum_{j_{1}, j_{2}=1}^{N} \sum_{s_{1}, s_{2}=1}^{L} \delta\left(\boldsymbol{r}-\boldsymbol{r}_{j_{1}}\left(s_{1}\right)\right) U\left(\left|\boldsymbol{r}-\boldsymbol{r}^{\prime}\right|\right) \delta\left(\boldsymbol{r}^{\prime}-\boldsymbol{r}_{j_{2}}\left(s_{2}\right)\right) \\
& =\frac{\kappa V^{2}}{4 \varrho_{0}} \int \mathrm{d}^{d} r \int \mathrm{d}^{d} r^{\prime} \varrho(\boldsymbol{r}) U\left(\left|\boldsymbol{r}-\boldsymbol{r}^{\prime}\right|\right) \varrho\left(\boldsymbol{r}^{\prime}\right)
\end{aligned}
$$

with a first field, the local, total segment density 5

$$
\varrho(\boldsymbol{r}):=\frac{1}{V} \sum_{j=1}^{N} \sum_{s=1}^{L} \delta\left(\boldsymbol{r}-\boldsymbol{r}_{j}(s)\right)=\varrho_{A}(\boldsymbol{r})+\varrho_{B}(\boldsymbol{r}),
$$

i.e., the sum of type- $A$ and type- $B$ segment densities $\varrho_{A}$ and $\varrho_{B}$. Given that $U \geq 0$, the excluded volume interaction is seen to suppress spatial variations of the total segment density, eq. 2.27.

- The incompatibility interaction, eq. $(2.26 \mathrm{c})$, can be reformulated in analogy to eq. (2.27) as

$$
\begin{aligned}
\mathcal{H}_{\chi} & =-\frac{\chi V^{2}}{\varrho_{0}} \int \mathrm{d}^{d} r \int \mathrm{d}^{d} r^{\prime}\left[\varrho_{A}(\boldsymbol{r})-\frac{1+q}{2} \varrho(\boldsymbol{r})\right] W\left(\left|\boldsymbol{r}-\boldsymbol{r}^{\prime}\right|\right)\left[\frac{1-q}{2} \varrho\left(\boldsymbol{r}^{\prime}\right)-\varrho_{B}\left(\boldsymbol{r}^{\prime}\right)\right] \\
& =:-\frac{\chi V^{2}}{4 \varrho_{0}} \int \mathrm{d}^{d} r \int \mathrm{d}^{d} r^{\prime} \sigma(\boldsymbol{r}) W\left(\left|\boldsymbol{r}-\boldsymbol{r}^{\prime}\right|\right) \sigma\left(\boldsymbol{r}^{\prime}\right),
\end{aligned}
$$

with a second field that is sensitive to the fixed types of the segments, the local excess of $A$ segment: 6 ,

$$
\sigma(\boldsymbol{r}):=\frac{1}{V} \sum_{j=1}^{N} \sum_{s=1}^{L}\left(q_{j}(s)-q\right) \delta\left(\boldsymbol{r}-\boldsymbol{r}_{j}(s)\right)=(1-q) \varrho_{A}(\boldsymbol{r})-(1+q) \varrho_{B}(\boldsymbol{r}),
$$

or the weighted difference of segment densities due to $A$ and $B$. The ensemble average of this density field detects phase separation into $A$ - and $B$-rich domains and has been introduced as an order parameter in refs. [76, 42]. Note that in

\footnotetext{
${ }^{5}$ A differentiable, coarse-grained density field would consist of, e.g., Gaussians instead of $\delta$ peaks.

${ }^{6}$ An alternative approach distinguishes $A$ segment densities produced by different sequences [32, 7,6 ,
} 
our model the incompatibility potential eq. (2.21), respectively eq. (2.29), is zero for the state of uniform densities, in which $\varrho_{A}$ and $\varrho_{B}$ have the constant values $(1+q) / 2$ and $(1-q) / 2$ equal to the global $A$ and $B$ fractions. Since $W \geq 0$, the incompatibility promotes spatial inhomogeneities of $\sigma$.

Via the interplay of annealed positions and frozen-in types of the copolymer segments, mean-field modes of $\varrho$ and $\sigma$ will turn out to be coupled for nearly incompressible melts. 



\section{Free energy and global equilibrium states}

In this chapter, we analyze A-B phase separation in random, binary block copolymer melts starting from the canonical partition function. First, we derive a single-chain, effective Hamiltonian in terms of collective densities and conjugated interaction fields. The evaluation at the saddle point in the incompressible limit results in a free-energy functional of an order-parameter field indicating A-B separation. An expansion of the functional will show that a second-order structure function is decisive for the wave number and thus the morphology of a global phase-separated state which appears when increasing the incompatibility $\chi$ in the disordered state. This structure function is related to the mean-field scattering function of the homogeneous melt. In a symmetric, Markovian sequence distribution, either macroscopic or structured, lamellar phase separation takes place (cf. fig. 3.1), depending on the block-type correlation $\lambda$. The multicritical point $\left(\lambda_{c}, \chi_{c}\right)$, which separates macroscopic and lamellar phase separation, is found to change qualitatively with $M$, the number of segments per block, for Markovian triblock copolymers. In preparation for the analysis of fractionation in the next chapter, we calculate the free-energy densities of the ordered states and specify the dependence on the sequence probabilities in an $A \leftrightharpoons B$ exchange-symmetric, but otherwise arbitrary distribution. For random sequences with a small number $Q$ of blocks per chain, i.e., for melts with a small degree of sequence polydispersity, we can compute explicitly the contributions of individual sequences. With these expressions at hand, we will go on in chapter 4 to identify in addition to the global, ordered states a new state, viz. the coexistence of three phases, an A-rich one, a B-rich one, both homogeneous, and a lamellar phase. This coexistence will be shown to be enabled by sequence fractionation, i.e., the occurrence of subsystems with sequence distributions different from the global ( $\lambda$-defined) distribution.

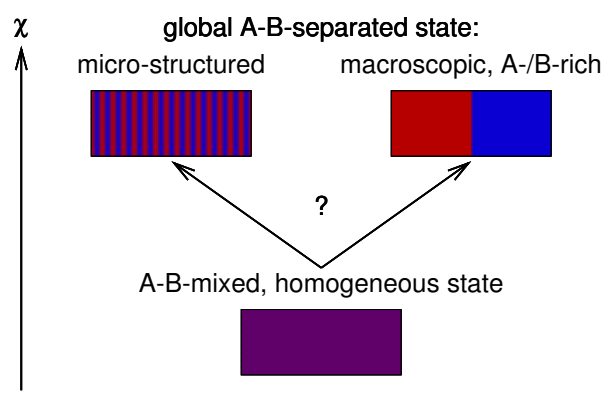

Figure 3.1: First instability of the mixed state towards a global $A$ - $B$-separated state with microor macroscopic $A$ - and $B$-rich domains. 


\subsection{Free-energy functional}

\subsubsection{Partition function}

Our derivation of the free-energy functional is presented in some detail in this section, in order to create a sound basis for the sequence fractionation ansatz which follows. With the contributions eqs. (2.26) to the total Hamiltonian $\mathcal{H}$, we set up the normalized canonical partition function

$$
\mathcal{Z}=\frac{\frac{1}{\prod_{\nu} N_{\nu} !} \int \mathcal{D}\left[\boldsymbol{r}_{j}(s)\right] \mathrm{e}^{-\mathcal{H}\left(\left\{q_{j}(s)\right\},\left\{\boldsymbol{r}_{j}(s)\right\}\right)}}{\frac{1}{N !} \int \mathcal{D}\left[\boldsymbol{r}_{j}(s)\right] \mathrm{e}^{-\mathcal{H}_{\mathrm{C}}\left(\left\{\boldsymbol{r}_{j}(s)\right\}\right)}} .
$$

The symbol $\mathcal{D}\left[\boldsymbol{r}_{j}(s)\right]$ denotes the measure of the $N L d$-fold integration over the segment coordinates (in the case of continuous-chain polymers, the functional integrations over the contours $\left.\boldsymbol{r}_{j}(s), s \in[0,1]\right)$. The system referred to in the numerator consists of $N=\sum_{\nu} N_{\nu}$ interacting block copolymers, where $N_{\nu}$ is the number of copolymers of block-type sequence $\nu$. A sequence $\nu$ is characterized by the set of (blockwise equal) segment types $\left\{q_{\nu}(s)\right\}_{s=1}^{L}$. Assuming copolymers of identical block-type sequence to be indistinguishable (although a polymer is a particle with internal interactions), the combinatorial prefactor $1 / \prod_{\nu}\left(N_{\nu}\right)$ ! reflects the usual over-counting correction in the partition function [46]. The partition function in the denominator is a product of singlechain partition functions of $N$ identical, non-interacting Gaussian chains (cf. eq. 2.26a)), the factor $1 / N$ ! again accounting for indistinguishability. The normalization allows us to calculate from $\mathcal{Z}$ the free energy of the block copolymer melt relative to that of a disordered, single-component polymer melt with uniform densities and with no interactions but connectivity. In the following,

$$
\Omega:=\frac{1}{N !} \prod_{\nu}\left(N_{\nu}\right) !
$$

will be used as a shorthand for the combinatorial prefactors. The contributions due to eq. (3.2) will become important for a coexistence of phases in subsystems with different sequence distributions, which we will analyze in chap. 4 .

In the thermodynamic limit, a set of probabilities $\left\{p_{\nu}:=N_{\nu} / N\right\}$ defines the distribution over the different sequences realized in a random, binary $Q$-block copolymer (see eq. (2.6) for the actual number $\mathcal{N}(Q)$ of different sequences). For a Markovian distribution with $A \leftrightharpoons B$ exchange symmetry, $p=1 / 2$ or $q=0$, the $p_{\nu}$ will be defined solely by the block-type correlation parameter $\lambda$. Owing to our focus on a copolymer distribution with a finite number $\mathcal{N}$ of sequences, we can avoid to employ the replica method, which is often used to address a system with quenched disorder [125]. A brief note on the self-averaging properties of this system may be in order here. The model of random block-type sequences with the incompatibility interaction eq. (2.26c), in which the interaction between a pair of segments depends on their types, corresponds to site disorder rather than to, e.g., bond randomness in the Sherrington-Kirkpatrick model of spin glasses [84, 92]. With short-ranged non-bonded potentials, self-averaging of the free energy can be expected [78, 112]. 
In order to detect an instability toward a spatially structured, ordered phase, a Fourier representation of the fields that determine the free-energy functional proves to be convenient. The Fourier modes $\sigma_{\boldsymbol{k}}$ and $\varrho_{\boldsymbol{k}}$ of the collective $A$-excess density and the total segment density (cf. eqs. (2.30) and $(2.28)$ ) are, respectively,

$$
\sigma_{\boldsymbol{k}}=\frac{1}{V} \sum_{j=1}^{N} \sum_{s=1}^{L}\left(q_{j}(s)-q\right) \mathrm{e}^{i \boldsymbol{k} \cdot \boldsymbol{r}_{j}(s)} \text { and } \varrho_{\boldsymbol{k}}=\frac{1}{V} \sum_{j=1}^{N} \sum_{s=1}^{L} \mathrm{e}^{i \boldsymbol{k} \cdot \boldsymbol{r}_{j}(s)}
$$

and, following eqs. (2.27) and (2.29), the non-bonded parts of the Hamiltonian read

$$
\mathcal{H}_{\chi}+\mathcal{H}_{\mathrm{ev}}=-\frac{N L \chi}{4 \varrho_{0}^{2}} \sum_{\boldsymbol{k} \neq \mathbf{0}} \sigma_{\boldsymbol{k}} W_{\boldsymbol{k}} \sigma_{-\boldsymbol{k}}+\frac{N L \kappa}{4 \varrho_{0}^{2}} \sum_{\boldsymbol{k} \neq \mathbf{0}} \varrho_{\boldsymbol{k}} U_{\boldsymbol{k}} \varrho_{-\boldsymbol{k}},
$$

where $W_{k}, U_{k}$ are the Fourier-transformed shape functions of incompatibility and excluded volume interaction, respectively. The average segment density is $\varrho_{0}=N L / V$. By definition of the densities and potential shapes, $\sigma_{-\boldsymbol{k}}$ is the complex conjugate, $\sigma_{\boldsymbol{k}}^{*}$, of $\sigma_{\boldsymbol{k}}$, analogously for $\varrho_{\boldsymbol{k}}, W_{\boldsymbol{k}}$ and $U_{\boldsymbol{k}}$. Since the imaginary parts thus cancel in eq. (3.4), we can assume $W_{\boldsymbol{k}}, U_{\boldsymbol{k}}$ to be real-valued. For a finite total volume $V=L_{V}^{d}$, the allowed wavevectors have components $k_{\alpha} \in \frac{2 \pi}{L_{V}} \mathbb{Z}, \alpha=1, \ldots, d$. Fourier transforms of smooth densities and potentials drop to zero at large wave numbers $k:=|\boldsymbol{k}|$, such that the sum over $\boldsymbol{k}$ is actually bounded 11 Then, the partition function eq. (3.1) can be expressed as

$$
\begin{aligned}
\mathcal{Z} & =\frac{1}{\Omega}\left\langle\exp \left\{\frac{N L}{4 \varrho_{0}^{2}} \sum_{\boldsymbol{k} \neq \mathbf{0}}\left(\chi W_{\boldsymbol{k}}\left|\sigma_{\boldsymbol{k}}\right|^{2}-\kappa U_{\boldsymbol{k}}\left|\varrho_{\boldsymbol{k}}\right|^{2}\right)\right\}\right\rangle_{\mathcal{H}_{\mathrm{C}}} \\
& =\frac{1}{\Omega}\left\langle\exp \left\{\frac{N L}{4 \varrho_{0}^{2}} \sum_{\boldsymbol{k} \neq \mathbf{0}}\left(\chi W_{\boldsymbol{k}}\left[\left(\operatorname{Re} \sigma_{\boldsymbol{k}}\right)^{2}+\left(\operatorname{Im} \sigma_{\boldsymbol{k}}\right)^{2}\right]-\kappa U_{\boldsymbol{k}}\left[\left(\operatorname{Re} \varrho_{\boldsymbol{k}}\right)^{2}+\left(\operatorname{Im} \varrho_{\boldsymbol{k}}\right)^{2}\right]\right)\right\}\right\rangle_{\mathcal{H}_{\mathrm{C}}}
\end{aligned}
$$

with the connectivity average for $N$ Gaussian chains,

$$
\langle\cdot\rangle_{\mathcal{H}_{\mathrm{C}}}:=\frac{\int \mathcal{D}\left[\boldsymbol{r}_{j}(s)\right](\cdot) \exp \left\{-\frac{1}{4} \sum_{j=1}^{N} \sum_{s=1}^{L}\left(\boldsymbol{r}_{j}(s)-\boldsymbol{r}_{j}(s-1)\right)^{2}\right\}}{\int \mathcal{D}\left[\boldsymbol{r}_{j}(s)\right] \exp \left\{-\frac{1}{4} \sum_{j=1}^{N} \sum_{s=1}^{L}\left(\boldsymbol{r}_{j}(s)-\boldsymbol{r}_{j}(s-1)\right)^{2}\right\}},
$$

called conformational average in the following.

\subsubsection{Hubbard-Stratonovich transformation}

In order to linearize the exponent in (3.5) in $\varrho_{\boldsymbol{k}}$ and $\sigma_{\boldsymbol{k}}$ and thus to arrive at a product of single-chain partition functions, we make use of the Hubbard-Stratonovich (HS) transformation [116, 64] (see appendix A.1). This method couples the collective density modes of mutually non-interacting chains to the modes of conjugated fields $\hat{\sigma}$ and $\hat{\varrho}$, called interaction fields. In mean-field theory, they will be determined self-consistently

\footnotetext{
${ }^{1}$ Consistently, the field theory applied here, which rests on smooth functions, cannot make predictions on molecular scales.
} 
by the collective densities due to all chains. Due to the symmetry of the Fouriertransformed densities and potential shapes, interaction fields must strictly be introduced only for the set of $\boldsymbol{k}$-vectors in a half-space, $\boldsymbol{k} \cdot \boldsymbol{e}>0$, with an arbitrary unit vector $\boldsymbol{e}$. Since we are free to define interaction fields for all $\boldsymbol{k}$, we introduce real-valued integration variables $\hat{\sigma}_{\boldsymbol{k}}^{\prime}, \hat{\sigma}_{\boldsymbol{k}}^{\prime \prime}, \varrho_{\boldsymbol{k}}^{\prime}, \hat{\varrho}_{\boldsymbol{k}}^{\prime \prime}$, to be the components of complex fields $\hat{\varrho}, \hat{\sigma}$ (cf. appendix A.1) as follows:

$$
\hat{\sigma}_{\boldsymbol{k}}:=\left\{\begin{array}{ll}
\hat{\sigma}_{\boldsymbol{k}}^{\prime}+i \hat{\sigma}_{\boldsymbol{k}}^{\prime \prime}, & \boldsymbol{k} \cdot \boldsymbol{e}>0, \\
\hat{\sigma}_{\boldsymbol{k}}^{\prime}-i \hat{\sigma}_{\boldsymbol{k}}^{\prime \prime}, & \boldsymbol{k} \cdot \boldsymbol{e}<0,
\end{array} \quad \hat{\varrho}_{\boldsymbol{k}}:= \begin{cases}\hat{\varrho}_{\boldsymbol{k}}^{\prime}+i \hat{\varrho}_{\boldsymbol{k}}^{\prime \prime}, & \boldsymbol{k} \cdot \boldsymbol{e}>0 \\
\hat{\varrho}_{\boldsymbol{k}}^{\prime}-i \hat{\varrho}_{\boldsymbol{k}}^{\prime \prime}, & \boldsymbol{k} \cdot \boldsymbol{e}<0 .\end{cases}\right.
$$

For convenience in the following transformation and without loss of generality, we restrict the discussion to potential shapes with $W_{\boldsymbol{k}} \geq 0, U_{\boldsymbol{k}} \geq 0$ for all $\boldsymbol{k}$ (e.g., Gaussians). In the HS-transformed partition function, the terms originally quadratic in the collective densities are reflected in Gaussian probability distributions of the interaction fields:

$$
\begin{aligned}
\mathcal{Z}=\frac{1}{\Omega} & \left\langle\int \mathcal{D}[\hat{\sigma}] \int \mathcal{D}[\hat{\varrho}] \exp \left\{-\frac{N L}{4 \varrho_{0}^{2}} \sum_{\boldsymbol{k} \neq \mathbf{0}}\left(\chi W_{\boldsymbol{k}} \hat{\sigma}_{\boldsymbol{k}} \hat{\sigma}_{-\boldsymbol{k}}+\kappa U_{\boldsymbol{k}} \hat{\varrho}_{\boldsymbol{k}} \hat{\varrho}_{-\boldsymbol{k}}\right)\right\}\right. \\
& \left.\times \exp \left\{\frac{N L}{2 \varrho_{0}^{2}} \sum_{\boldsymbol{k} \neq \mathbf{0}}\left[\chi W_{\boldsymbol{k}} \operatorname{Re}\left(\hat{\sigma}_{\boldsymbol{k}} \sigma_{\boldsymbol{k}}^{*}\right)+i \kappa U_{\boldsymbol{k}} \operatorname{Re}\left(\hat{\varrho}_{\boldsymbol{k}} \varrho_{\boldsymbol{k}}^{*}\right)\right]\right\}\right\rangle_{\mathcal{H}_{\mathrm{C}}},
\end{aligned}
$$

with the shorthands for the integrations along the real axis $\mathbb{R}$,

$$
\int \mathcal{D}[\hat{\sigma}]:=\prod_{\boldsymbol{k}} \frac{N L \chi W_{\boldsymbol{k}}}{2 \pi \varrho_{0}^{2}} \int_{\mathbb{R}} \mathrm{d} \hat{\sigma}_{\boldsymbol{k}}^{\prime} \int_{\mathbb{R}} \mathrm{d} \hat{\sigma}_{\boldsymbol{k}}^{\prime \prime} \text { and } \int \mathcal{D}[\hat{\varrho}]:=\prod_{\boldsymbol{k}} \frac{N L \kappa U_{\boldsymbol{k}}}{2 \pi \varrho_{0}^{2}} \int_{\mathbb{R}} \mathrm{d} \hat{\varrho}_{\boldsymbol{k}}^{\prime} \int_{\mathbb{R}} \mathrm{d} \hat{\varrho}_{\boldsymbol{k}}^{\prime \prime} .
$$

(The real part operations in eq. (3.8) are strictly redundant due to the symmetries of the densities and the interaction fields.) Given that the interaction fields do not contain any chain-specific variables, integrations over the segment positions $\left\{\boldsymbol{r}_{j_{\nu}}(s)\right\}$ are seen to affect the second line of eq. (3.8) only and hence can be interchanged with the integrations over the interaction fields. By virtue of the HS transformations, the Fourier modes of the $A$ excess $\sigma$ and the total segment density $\varrho$, eq. (3.3), now appear linear in the exponent of eq. (3.8) and can be conveniently decomposed into single-chain contributions, grouped according to the block-type sequence $\nu$ :

$$
\sigma_{\boldsymbol{k}}=\frac{1}{V} \sum_{\nu} \sum_{j_{\nu}=1}^{N_{\nu}} \sum_{s=1}^{L}\left(q_{\nu}(s)-q\right) \mathrm{e}^{i \boldsymbol{k} \cdot \boldsymbol{r}_{j_{\nu}}(s)} \text { and } \varrho_{\boldsymbol{k}}=\frac{1}{V} \sum_{\nu} \sum_{j_{\nu}=1}^{N_{\nu}} \sum_{s=1}^{L} \mathrm{e}^{i \boldsymbol{k} \cdot \boldsymbol{r}_{j_{\nu}}(s)} .
$$

This allows us to exploit the fact that the conformational average eq. (3.6) is a product of single-chain weights, whereby the second line of eq. (3.8) factorizes. Consequently, in

$$
\mathcal{Z}=\frac{1}{\Omega} \int \mathcal{D}[\hat{\sigma}] \int \mathcal{D}[\varrho] \exp \left\{-\frac{N L}{4 \varrho_{0}^{2}} \sum_{\boldsymbol{k} \neq \mathbf{0}}\left(\chi W_{\boldsymbol{k}} \hat{\sigma}_{\boldsymbol{k}} \hat{\sigma}_{-\boldsymbol{k}}+\kappa U_{\boldsymbol{k}} \hat{\varrho}_{\boldsymbol{k}} \hat{\varrho}_{-\boldsymbol{k}}\right)\right\} \prod_{\nu} \hat{z}_{\nu}^{N_{\nu}}
$$

all copolymers with sequence $\nu$ contribute the same single-chain partition function $\hat{z}_{\nu}$,

$$
\hat{z}_{\nu}[\hat{\sigma}, \hat{\varrho}]=\left\langle\exp \left\{\sum_{\boldsymbol{k} \neq \mathbf{0}} \sum_{s=1}^{L} \frac{1}{2 \varrho_{0}}\left(\chi W_{\boldsymbol{k}} \hat{\sigma}_{\boldsymbol{k}}\left(q_{\nu}(s)-q\right)+i \kappa U_{\boldsymbol{k}} \hat{\varrho}_{\boldsymbol{k}}\right) \mathrm{e}^{-i \boldsymbol{k} \cdot \boldsymbol{r}(s)}\right\}\right\rangle,
$$


where $\langle\cdot\rangle$ denotes the conformational average for a single Gaussian chain,

$$
\langle\cdot\rangle:=\lim _{V \rightarrow \infty} \frac{1}{V}(4 \pi)^{-L d / 2} \int \mathcal{D}[\boldsymbol{r}(s)](\cdot) \exp \left\{-\frac{1}{4} \sum_{s=1}^{L}(\boldsymbol{r}(s)-\boldsymbol{r}(s-1))^{2}\right\} .
$$

Dropping the subscript $j_{\nu}$ of a copolymer's coordinates in eq. 3.12 is possible, since our model assumes the same conformational statistics for all copolymers irrespective of their block-type sequence. With eqs. (3.11) and (3.12), the partition function $\mathcal{Z}$ can be cast into the form

$$
\mathcal{Z}=\int \mathcal{D}[\hat{\sigma}] \int \mathcal{D}[\hat{\varrho}] \exp \{-N \hat{h}[\hat{\sigma}, \hat{\varrho}]\}
$$

with the effective Hamiltonian $\hat{h}$ per polymer,

$$
\hat{h}[\hat{\sigma}, \hat{\varrho}]=\frac{L}{4 \varrho_{0}^{2}} \sum_{\boldsymbol{k} \neq \mathbf{0}}\left(\chi W_{\boldsymbol{k}} \hat{\sigma}_{\boldsymbol{k}} \hat{\sigma}_{-\boldsymbol{k}}+\kappa U_{\boldsymbol{k}} \hat{\varrho}_{\boldsymbol{k}} \hat{\varrho}_{-\boldsymbol{k}}\right)-\sum_{\nu} p_{\nu} \ln \frac{\hat{z}_{\nu}[\hat{\sigma}, \hat{\varrho}]}{p_{\nu}} .
$$

The combinatorial prefactor $\Omega$, eq. (3.2), has been evaluated by the usual Stirling approximation using $p_{\nu}=N_{\nu} / N$. Thereby, we have arrived at and will continue to analyze an expression in the interaction fields, not in the collective densities, contrasting with the procedere in, e.g., refs. 76, 42. We note that the exponent in the partition function eq. (3.14) is extensive, with the effective Hamiltonian eq. (3.15) being an analytic function of the interaction fields, which justifies the following saddle-point evaluation.

\subsubsection{Saddle-point approximation and effective Hamiltonian}

In comparison to the original expression eq. (3.5), the partition function in eqs. (3.11), (3.14) has not yet been approximated, but merely reformulated with interaction fields $\hat{\sigma}$ and $\hat{\varrho}$ that act equally on each copolymer, but may be nonuniform in space. In general, it is neither feasible to perform the integrations in eq. (3.14) nor to solve the set of implicit, coupled saddle-point equations for the interaction fields, which follow from the conditions $0=\left.\left(\partial \hat{h} / \partial \hat{\sigma}_{\boldsymbol{k}}\right)\right|_{\hat{\sigma}=\hat{\tau}}$ and $0=\left.\left(\partial \hat{h} / \partial \hat{\varrho}_{\boldsymbol{k}}\right)\right|_{\hat{\varrho}=\hat{\omega}}$, cf. eqs. 3.15) and 3.12 :

$$
\begin{aligned}
& \frac{\hat{\tau}_{\boldsymbol{k}}}{\varrho_{0}}=\sum_{\nu} p_{\nu}\left\langle\frac{1}{L} \sum_{s=1}^{L}\left(q_{\nu}(s)-q\right) \mathrm{e}^{i \boldsymbol{k} \cdot \boldsymbol{r}(s)}\right\rangle_{\hat{\mathcal{H}}_{\nu}}, \\
& \frac{\hat{\omega}_{\boldsymbol{k}}}{\varrho_{0}}=\sum_{\nu} p_{\nu}\left\langle\frac{1}{L} \sum_{s=1}^{L} i \mathrm{e}^{i \boldsymbol{k} \cdot \boldsymbol{r}(s)}\right\rangle_{\hat{\mathcal{H}}_{\nu}}
\end{aligned}
$$

with modified single-chain averages $\langle\cdot\rangle_{\hat{\mathcal{H}}_{\nu}}$ according to

$$
\hat{\mathcal{H}}_{\nu}[\hat{\tau}, \hat{\omega}]:=\mathcal{H}_{\mathrm{C}, \text { s.c. }}-\frac{1}{2 \varrho_{0}} \sum_{\boldsymbol{k} \neq \mathbf{0}} \sum_{s}\left(\chi W_{\boldsymbol{k}}\left(q_{\nu}(s)-q\right) \hat{\tau}_{\boldsymbol{k}}+i \kappa U_{\boldsymbol{k}} \hat{\omega}_{\boldsymbol{k}}\right) \mathrm{e}^{-i \boldsymbol{k} \cdot \boldsymbol{r}(s)} .
$$

Here and in the following, $\hat{\tau}$ and $\hat{\omega}$ denote the saddle-point values of the interaction fields $\hat{\sigma}$ and $\hat{\varrho}$. At the instability of the disordered homogeneous $(A$ - $B$-mixed) state, in which the collective densities are uniform, $\sigma_{\boldsymbol{k}}=0, \varrho_{\boldsymbol{k}}=0$ for $\boldsymbol{k} \neq \mathbf{0}$, the saddle-point values 
$\hat{\tau}_{\boldsymbol{k}}$ and $\hat{\omega}_{\boldsymbol{k}}$ must vanish, to ${ }^{2}$. This follows, since by inspection of eqs. (3.5) and (3.11), averages of the interaction fields and of the collective densities are proportional to each other, as we show for the averages of $\hat{\sigma}$ and $\sigma$ (which are identical) in appendix A.3.1 Therefore, in the vicinity of the saddle-point values near the instability, we may expand the effective Hamiltonian eq. 3.15 in powers of the fields $\hat{\sigma}$ and $\hat{\varrho}$ and truncate the resulting series ${ }^{3}$.

In doing so, we specialize to systems for which the phase transition to an $A-B$ separated state is continuous. Within mean-field theory, continuous phase transitions can be expected if the expansion contains only vertices of even order in $\hat{\sigma}$ (see, e.g., [76, 9, 48]). For our expansion, this can be shown for block-type sequence distributions, which are globally invariant under $A \leftrightharpoons B$ exchange, such that a sequence $\nu$ and its complement $\bar{\nu}$, cf. the definition in appendix B.1, have equal concentrations. The restriction to Markovian sequences is not necessary at this point. Subsystems with altered sequence distributions, created by fractionation, which we will analyze later, must display the $A \leftrightharpoons B$ symmetry of the global distribution, too.

In the following, the short-ranged shape functions $W(r)$ and $U(r)$ of the non-bonded interactions will be approximated by $\delta$-functions, $U_{\boldsymbol{k}}=W_{\boldsymbol{k}}=1$, since our focus is on $A$-density modulations with wavelengths large compared to the typical range of segment interactions. Moreover, this limit eliminates any interaction radius and therefore seems to be consistent with the mean-field approximation.

Aiming at the saddle-point of the density interaction field $\hat{\omega}$ near the instability, we write out the expansion of eq. (3.15) first with consideration only for the lowest-order terms in $\hat{\varrho}$, i.e., up to second order in $\hat{\varrho}$ and up to combined third order in $\hat{\varrho}, \hat{\sigma}$ :

$$
\begin{aligned}
\hat{h}[\hat{\sigma}, \hat{\varrho}]= & \sum_{\nu} p_{\nu} \ln p_{\nu}+\frac{1}{8 \varrho_{0}^{2}} \sum_{\boldsymbol{k} \neq \mathbf{0}}\left(2 L \chi-\chi^{2} \sum_{\nu} p_{\nu} S_{\nu}\left(k^{2}\right)\right) \hat{\sigma}_{\boldsymbol{k}} \hat{\sigma}_{-\boldsymbol{k}} \\
& +\frac{\kappa^{2}}{8 \varrho_{0}^{2}} \sum_{\boldsymbol{k} \neq \mathbf{0}}\left(\frac{2 L}{\kappa}+D\left(L, k^{2}\right)\right) \hat{\varrho}_{\boldsymbol{k}} \hat{\varrho}_{-\boldsymbol{k}} \\
& -i \frac{\chi^{2} \kappa}{16 \varrho_{0}^{3}} \sum_{\substack{\boldsymbol{k}_{1}, \boldsymbol{k}_{2} \neq \mathbf{0}, \boldsymbol{k}_{2} \neq \boldsymbol{k}_{1}}} \sum_{\nu} p_{\nu} S_{\nu}^{(\alpha)}\left(\boldsymbol{k}_{1}, \boldsymbol{k}_{2}-\boldsymbol{k}_{1}\right) \hat{\sigma}_{\boldsymbol{k}_{1}} \hat{\sigma}_{\boldsymbol{k}_{2}-\boldsymbol{k}_{1}} \hat{\varrho}_{-\boldsymbol{k}_{2}} \\
& +\mathcal{O}\left(\hat{\varrho}_{\boldsymbol{k}}^{3}, \hat{\varrho}_{\boldsymbol{k}}^{2} \hat{\sigma}_{\boldsymbol{k}}^{2}, \hat{\sigma}_{\boldsymbol{k}}^{4}, \ldots\right),
\end{aligned}
$$

In eq. (3.18), we have introduced symbols for structure or vertex functions, which are based on Gaussian-chain averages of exponentials (cf. appendix C.1),

$$
\left\langle\exp \left\{-i \sum_{\mathrm{r}=1}^{n} \boldsymbol{k}_{\mathrm{r}} \cdot \boldsymbol{r}\left(s_{\mathrm{r}}\right)\right\}\right\rangle=\delta_{\sum_{\mathrm{r}} \boldsymbol{k}_{\mathrm{r}}, \mathbf{0}} \exp \left\{\sum_{\mathrm{r}<\mathrm{r} \prime}\left|s_{\mathrm{r}}-s_{\mathrm{r} /}\right| \boldsymbol{k}_{\mathrm{r}} \cdot \boldsymbol{k}_{\mathrm{r} \prime}\right\},
$$

and in principle can be computed (the effort depending on the sequence distribution):

\footnotetext{
${ }^{2}$ The trivial solution to eqs. $\sqrt{3.16 \mathrm{a})}$ is $\hat{\tau}_{\boldsymbol{k}}=0, \hat{\omega}_{\boldsymbol{k}} \propto \delta_{\boldsymbol{k}, \mathbf{0}}$.

${ }^{3}$ The limit of small field amplitudes and gradients is often termed the 'weak inhomogeneities' or 'weak segregation' limit [76, 40, 5, 39.
} 
The symbol $S_{\nu}\left(k^{2}\right)$ denotes the second-order structure function for sequence $\nu$,

$$
\begin{aligned}
S_{\nu}\left(k^{2}\right) & :=\sum_{s_{1}, s_{2}=1}^{L} q_{\nu}\left(s_{1}\right) q_{\nu}\left(s_{2}\right)\left\langle\mathrm{e}^{-i\left(\boldsymbol{k}_{1} \cdot \boldsymbol{r}\left(s_{1}\right)+\boldsymbol{k}_{2} \cdot \boldsymbol{r}\left(s_{2}\right)\right)}\right\rangle \\
& =\sum_{s_{1}, s_{2}=1}^{L} q_{\nu}\left(s_{1}\right) q_{\nu}\left(s_{2}\right) \mathrm{e}^{\left|s_{2}-s_{1}\right| \boldsymbol{k}_{1} \cdot \boldsymbol{k}_{2}} \delta_{-\boldsymbol{k}_{2}, \boldsymbol{k}_{1}=: \boldsymbol{k}} \\
& =\sum_{s_{1}, s_{2}=1}^{L} q_{\nu}\left(s_{1}\right) q_{\nu}\left(s_{2}\right) \mathrm{e}^{-\left|s_{2}-s_{1}\right| k^{2}}
\end{aligned}
$$

Particularly for homopolymers (hp), for which $q_{\mathrm{hp}}(s)$ has the same value for all $s$, $S_{\mathrm{hp}}\left(k^{2}\right)$ is denoted as

$$
D\left(L, k^{2}\right):=\sum_{s_{1}, s_{2}=1}^{L} \mathrm{e}^{-\left|s_{2}-s_{1}\right| k^{2}} .
$$

since at least the continuous-chain version is known as the Debye function [24. The three-point structure function $S_{\nu}^{(\alpha)}$ is defined as

$$
S_{\nu}^{(\alpha)}\left(\boldsymbol{k}_{1}, \boldsymbol{k}_{2}\right):=\sum_{s_{1}, s_{2}, s_{3}=1}^{L} q_{\nu}\left(s_{1}\right) q_{\nu}\left(s_{2}\right)\left\langle\mathrm{e}^{-i\left(\boldsymbol{k}_{1} \cdot \boldsymbol{r}\left(s_{1}\right)+\boldsymbol{k}_{2} \cdot \boldsymbol{r}\left(s_{2}\right)+\boldsymbol{k}_{3} \cdot \boldsymbol{r}\left(s_{3}\right)\right)}\right\rangle .
$$

Explicit calculations of the structure functions will be relegated to sec. 3.3 .3 and appendices C.2 to C.6. In the following, symbols $S$ without subscript $\nu$ denote averages of the respective structure functions over the given sequence distribution $\left\{p_{\nu}\right\}$, such as for the second-order structure function,

$$
S\left(k^{2}\right):=\sum_{\nu} p_{\nu} S_{\nu}\left(k^{2}\right)=\sum_{\nu} p_{\nu} \sum_{s_{1}, s_{2}=1}^{L} q_{\nu}\left(s_{1}\right) q_{\nu}\left(s_{2}\right) \mathrm{e}^{-\left|s_{2}-s_{1}\right| k^{2}} .
$$

For Markovian ( $\lambda$-defined) sequences, the averages $\sum_{\nu} p_{\nu}(\lambda) q_{\nu}\left(s_{1}\right) q_{\nu}\left(s_{2}\right) \cdots q_{\nu}\left(s_{n}\right)$ can be computed in closed form as a function of $\lambda$ and the set of segment distances in blocks, $\left\{\Delta \beta\left(s_{i}, s_{j}\right)\right\}$, cf. appendix B.3. which allows for the calculation of the global structure function without specifying the contribution of each sequence. In order to allow for the coexistence of arbitrary (symmetric) distributions in subsystems, however, we will have to compute the functions for individual sequences. The physical meaning of $S\left(k^{2}\right)$ and its rôle for the phase behavior, especially for Markovian block copolymers, will be analyzed in detail in section 3.3 .

Returning to the set of saddle-point equations for the density interaction amplitudes, $0=\left.\left(\partial \hat{h} / \partial \hat{\varrho}_{\boldsymbol{k}}\right)\right|_{\hat{\varrho}=\hat{\omega}}$, derived from the expansion eq. 3.18 , we obtain

$$
\kappa \hat{\omega}_{\boldsymbol{k}}=i \frac{\chi^{2}}{4 \varrho_{0}} \sum_{\boldsymbol{k}_{1} \notin\{\mathbf{0}, \boldsymbol{k}\}} \frac{S^{(\alpha)}\left(\boldsymbol{k}_{1}, \boldsymbol{k}-\boldsymbol{k}_{1}\right)}{\frac{2 L}{\kappa}+D\left(L, k^{2}\right)} \hat{\tau}_{\boldsymbol{k}_{1}} \hat{\tau}_{\boldsymbol{k}-\boldsymbol{k}_{1}}+\mathcal{O}\left(\hat{\tau}_{\boldsymbol{k}}^{4}\right), \boldsymbol{k} \neq \mathbf{0},
$$

i.e., to lowest order, a quadratic dependence on the (as yet undetermined) saddlepoint amplitudes $\hat{\tau}_{\boldsymbol{k}}$ of the $A$ excess interaction field. The excluded volume interaction 
with strength $\kappa$, eq. (2.19), had been introduced in our model merely to prevent unbounded spatial variations of the total density, which might arise from energetically favored compression of $A$ - or $B$-rich regions (see fig. 3.2 and the discussion in sec. 3.1 .4 below). Now, insertion of eq. (3.24) into the expansion eq. (3.18) eliminates the density interaction modes at the saddle point, $\kappa \hat{\omega}_{\boldsymbol{k}}$, in favor of $\hat{\tau}_{\boldsymbol{k}}$ and produces positive terms of fourth order in $\hat{\tau}$, essential for the positive definiteness of the complete fourth-order terms. Consequently, the density interaction modes do not influence the instability of the disordered, homogenous state (cf. sec. 3.2), but assure the stability of an $A-B$ phase-separated state. Even the incompressible limit, $\kappa \rightarrow \infty$, which enforces uniform total segment density and thus $\hat{\omega}_{\boldsymbol{k}} \rightarrow 0, \boldsymbol{k} \neq \mathbf{0}$, does not qualitatively change the situation in that the product $\kappa \hat{\omega}_{\boldsymbol{k}}$ gives a finite contribution. The remaining terms $\propto \hat{\tau}_{\boldsymbol{k}}^{4}$ have to be extracted from eq. 3.15 to arrive at a consistent expansion in $\hat{\tau}_{\boldsymbol{k}}$ up to fourth order. This yields, in the incompressible limit $\kappa \rightarrow \infty$, the free-energy functional per chain

$$
\begin{aligned}
& f[\hat{\tau}]=\sum_{\nu} p_{\nu} \ln p_{\nu}+\frac{(L \chi)^{2}}{4 \varrho_{0}^{2}} \sum_{\boldsymbol{k}}^{\prime}\left(\frac{1}{L \chi}-\frac{S\left(k^{2}\right)}{2 L^{2}}\right) \hat{\tau}_{\boldsymbol{k}} \hat{\tau}_{-\boldsymbol{k}} \\
& +\frac{(L \chi)^{4}}{3 \cdot 2^{7} \varrho_{0}^{4}}\left\{3 \sum_{\boldsymbol{k}_{1}, \boldsymbol{k}_{2} \neq-\boldsymbol{k}_{1}, \boldsymbol{k}_{3}}^{\prime} \frac{S^{(\alpha)}\left(\boldsymbol{k}_{1}, \boldsymbol{k}_{2}\right) S^{(\alpha)}\left(\boldsymbol{k}_{3},-\boldsymbol{k}_{1}-\boldsymbol{k}_{2}-\boldsymbol{k}_{3}\right)}{L^{4} D\left(L,\left(\boldsymbol{k}_{1}+\boldsymbol{k}_{2}\right)^{2}\right)}\right. \\
& \left.-\sum_{\boldsymbol{k}_{1}, \boldsymbol{k}_{2}, \boldsymbol{k}_{3}}^{\prime} \frac{S^{(\beta)}\left(\boldsymbol{k}_{1}, \boldsymbol{k}_{2}, \boldsymbol{k}_{3}\right)}{L^{4}}\right\} \hat{\tau}_{\boldsymbol{k}_{1}} \hat{\tau}_{\boldsymbol{k}_{2}} \hat{\tau}_{\boldsymbol{k}_{3}} \hat{\tau}_{-\boldsymbol{k}_{1}-\boldsymbol{k}_{2}-\boldsymbol{k}_{3}} \\
& +\frac{(L \chi)^{4}}{2^{7} \varrho_{0}^{4}} \sum_{\boldsymbol{k}_{1}, \boldsymbol{k}_{2} \neq 0} \frac{S^{(\gamma)}\left(k_{1}^{2}, k_{2}^{2}\right)}{L^{4}} \hat{\tau}_{\boldsymbol{k}_{1}} \hat{\tau}_{-\boldsymbol{k}_{1}} \hat{\tau}_{\boldsymbol{k}_{2}} \hat{\tau}_{-\boldsymbol{k}_{2}}+\mathcal{O}\left(\hat{\tau}_{\boldsymbol{k}}^{6}\right) \text {, }
\end{aligned}
$$

with the fourth-order structure functions

$$
\begin{aligned}
S^{(\beta)}\left(\boldsymbol{k}_{1}, \boldsymbol{k}_{2}, \boldsymbol{k}_{3}\right) & :=\sum_{\nu} p_{\nu} \sum_{s_{1}, s_{2}, s_{3}, s_{4}=1}^{L} q_{\nu}\left(s_{1}\right) q_{\nu}\left(s_{2}\right) q_{\nu}\left(s_{3}\right) q_{\nu}\left(s_{4}\right)\left\langle\mathrm{e}^{-i \sum_{\mathrm{r}=1}^{4} \boldsymbol{k}_{\mathrm{r}} \cdot \boldsymbol{r}\left(s_{\mathrm{r}}\right)}\right\rangle, \\
S^{(\gamma)}\left(k_{1}^{2}, k_{2}^{2}\right) & :=\sum_{\nu} p_{\nu} \sum_{s_{1}, s_{2}, s_{3}, s_{4}=1}^{L} q_{\nu}\left(s_{1}\right) q_{\nu}\left(s_{2}\right) q_{\nu}\left(s_{3}\right) q_{\nu}\left(s_{4}\right) \mathrm{e}^{-k_{1}^{2}\left|s_{2}-s_{1}\right|-k_{2}^{2}\left|s_{4}-s_{3}\right|},
\end{aligned}
$$

and the restricted sum

$$
\sum_{\boldsymbol{k}_{1}, \ldots, \boldsymbol{k}_{n}}^{\prime}():=\sum_{\substack{\boldsymbol{k}_{1}, \ldots, \boldsymbol{k}_{n} \neq \mathbf{0} \\ \sum_{\mathrm{r}=1}^{n} \boldsymbol{k}_{\mathrm{r}} \neq \mathbf{0}}}() .
$$

The functional eq. (3.25) still contains the amplitudes of $\hat{\tau}$, the saddle-point of the $A$ excess interaction field, which remain to be determined in order to extract the free-energy density of an $A-B$ phase-separated state. Consequently, at the saddle-point level, to which we adhere, we assign $\hat{\tau}$ the rôle of the order parameter, since the average of $\hat{\sigma}$ (which we can identify with the saddle-point value $\hat{\tau}$ ) and the average of $\sigma$ are identical, as mentioned earlier. However, the correlations of a collective density are not simply 
proportional to those of its interaction field. Particularly for the covariances of $\hat{\sigma}$ and $\sigma$, we have the relations

$$
\begin{aligned}
& \left\langle\sigma_{\boldsymbol{k}_{1}} \sigma_{-\boldsymbol{k}_{2}}\right\rangle_{\mathcal{H}}-\left\langle\sigma_{\boldsymbol{k}_{1}}\right\rangle_{\mathcal{H}}\left\langle\sigma_{-\boldsymbol{k}_{2}}\right\rangle_{\mathcal{H}} \\
= & \left\langle\hat{\sigma}_{\boldsymbol{k}_{1}} \hat{\sigma}_{-\boldsymbol{k}_{2}}\right\rangle_{N \hat{h}}-\left\langle\hat{\sigma}_{\boldsymbol{k}_{1}}\right\rangle_{N \hat{h}}\left\langle\hat{\sigma}_{-\boldsymbol{k}_{2}}\right\rangle_{N \hat{h}}-\frac{2 \varrho_{0}^{2}}{N L \chi} \delta_{\boldsymbol{k}_{1},-\boldsymbol{k}_{2}},
\end{aligned}
$$

where $\langle\cdot\rangle_{\mathcal{H}}$ is the canonical average, and $\langle\cdot\rangle_{N \hat{h}}$ is the average with the effective Hamiltonian eq. (3.15) from eq. (3.11); for details see appendix A.3.1. Therefore the vertices in eq. (3.25) differ from the vertices of an expansion in the A excess density $\sigma$, derived in refs. [76, 42] (apart from differences due to restrictions, e.g., to continuous chains with many blocks, which we do not impose), cf. appendix A.3.2.

Since at this stage we are interested in the instability of the disordered, homogeneously mixed state and its close vicinity, we can first neglect the wave-vector dependence in the coefficients of the terms $\propto \hat{\tau}_{k}^{4}$ in the functional eq. (3.25). This is in the spirit of a Landau free energy [48, chap. 5] and amounts to ignoring spatial variations of fourth-order correlations (in section 3.4.2, we will relax this approximation). Evaluation of the higher-order structure functions in the limit $\boldsymbol{k}_{\mathrm{r}} \rightarrow \mathbf{0}$ gives moments of composition ( $A$ excess) of the given sequence distribution $\left\{p_{\nu}\right\}$ (cf. appendix B.3). For arbitrary order $n$, these moments are defined as

$$
m_{n}:=\frac{1}{L^{n}} \sum_{\nu} p_{\nu} \sum_{s_{1}, \ldots, s_{n}=1}^{L} q_{\nu}\left(s_{1}\right) \ldots q_{\nu}\left(s_{n}\right), n \in \mathbb{N} .
$$

Particularly, $\left|m_{n}\right| \leq 1$ per definition of the $q(s)$, cf. eq. (2.2). The simplified quartic terms of eq. 3.25) feature the squared second and the fourth moment via the relationships

$$
\begin{aligned}
& m_{2}=S\left(k^{2}=0\right) / L^{2}=S^{(\alpha)}\left(\boldsymbol{k}_{1}=\mathbf{0}, \boldsymbol{k}_{2}=\mathbf{0}\right) / L^{3}, \\
& m_{4}=S^{(\beta)}\left(\boldsymbol{k}_{1}=\mathbf{0}, \boldsymbol{k}_{2}=\mathbf{0}, \boldsymbol{k}_{3}=\mathbf{0}\right) / L^{4}=S^{(\gamma)}\left(k_{1}^{2}=0, k_{2}^{2}=0\right) / L^{4}
\end{aligned}
$$

(in appendices B.3.1 and B.3.2, $m_{2}$ and $m_{4}$ are explicitly computed for Markovian sequence distributions). Observing the different restrictions on the sums in eq. (3.25), we obtain the simplified free-energy functional

$$
\begin{aligned}
& f_{0}\left[\hat{\tau}_{\boldsymbol{k}}\right]=\sum_{\nu} p_{\nu} \ln p_{\nu}+\frac{(L \chi)^{2}}{4 \varrho_{0}^{2}} \sum_{\boldsymbol{k}}^{\prime}\left(\frac{1}{L \chi}-\frac{S\left(k^{2}\right)}{2 L^{2}}\right) \hat{\tau}_{\boldsymbol{k}} \hat{\tau}_{-\boldsymbol{k}} \\
&+\frac{(L \chi)^{4}}{3 \cdot 2^{7} \varrho_{0}^{4}}\left\{\left(3 m_{2}^{2}-m_{4}\right) \sum_{\boldsymbol{k}_{1}, \boldsymbol{k}_{2} \neq-\boldsymbol{k}_{1}, \boldsymbol{k}_{3}}^{\prime} \hat{\tau}_{\boldsymbol{k}_{1}} \hat{\tau}_{\boldsymbol{k}_{2}} \hat{\tau}_{\boldsymbol{k}_{3}} \hat{\tau}_{-\boldsymbol{k}_{1}-\boldsymbol{k}_{2}-\boldsymbol{k}_{3}}\right. \\
&\left.+2 m_{4} \sum_{\boldsymbol{k}_{1}, \boldsymbol{k}_{2} \neq \mathbf{0}} \hat{\tau}_{\boldsymbol{k}_{1}} \hat{\tau}_{-\boldsymbol{k}_{1}} \hat{\tau}_{\boldsymbol{k}_{2}} \hat{\tau}_{-\boldsymbol{k}_{2}}\right\}+\mathcal{O}\left(\hat{\tau}_{\boldsymbol{k}}^{6}\right) .
\end{aligned}
$$

Again, we note that the term with coefficient $\propto m_{2}^{2}$ in eq. 3.32 , essential for the stability of the functional's expansion up to fourth order, stems from contributions of the density interaction field (hence, omitting from the start the density field in our description would have led to erroneous results even in the incompressible case). 


\subsubsection{Generalized density field modulations and asymmetric distributions}

The contributions eq. (3.24) of the density interaction field can be interpreted as the result of a reorganization process which necessarily involves both the order-parameter field and the total density interaction field: Incompatibility between $A$ and $B$ does not only favor domains where segments of one type accumulate, but also an increased total density in these $A$ - or $B$-rich domains, and a reduced density in the in-between $A$ - $B$-mixed regions. Given for instance a sinusoidal $A$ excess modulation about zero,

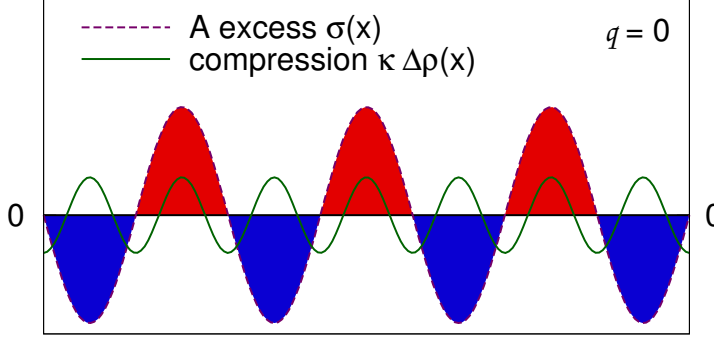

spatial coordinate $x$

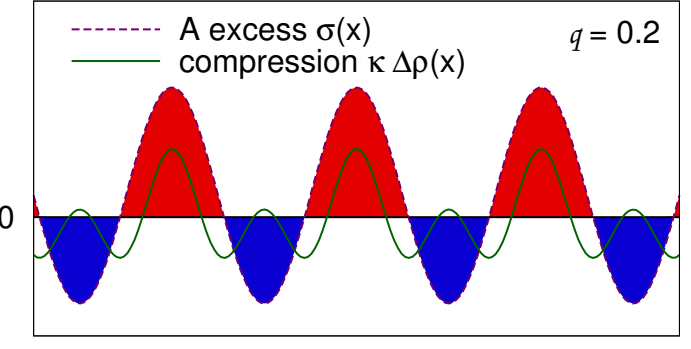

spatial coordinate $x$

Figure 3.2: Coupling of $A$ excess and generalized density/compression modes, for symmetric (left panel) and asymmetric (right panel) distributions of block copolymers.

such as in the left panel of fig. 3.2, the mode with half the wavelength of the $A$ excess remains for the generalized density fluctuations according to eq. (3.24). The $A \leftrightharpoons B$ exchange-symmetric distributions considered here constitute a 'critical plane' in the parameter space of global $A$ excess, block-type correlation, and incompatibility, $\{q, \lambda, \chi\}$. For asymmetric sequence distributions, modulations of $A$ excess and total segment density have equal periodicities (cf. the right panel of fig. 3.2), since domains with a large excess of only one segment type are separated by regions with a smaller excess. In this case, the lowest-order term in the equivalent of eq. 3.24 is linear in $\hat{\tau}_{\boldsymbol{k}}$, cf. also [125]. Accordingly, asymmetric distributions undergo first-order transitions to $A$ - $B$-separated states, as indicated also by mean-field theory [76, 18, 125]. A generalization of the second-order vertex of eq. $(3.25)$ and of $S\left(k^{2}\right)$, eq. (3.23), to arbitrary sequences or sequence distributions can be found in appendix A.2.

\subsection{Instability of the disordered state}

As mentioned above, we still have to optimize the free-energy functional eq. (3.32), respectively eq. (3.25), with respect to the order-parameter amplitudes $\hat{\tau}_{\boldsymbol{k}}$, which due to $\hat{\tau}=\langle\sigma\rangle_{\mathcal{H}}$ are those of the anticipated $A-B$ modulation. Granted that all coefficients of the free-energy functional are positive at sufficiently small incompatibilities $L \chi$, and that the fourth-order coefficients remain positive, a nonzero mode $\hat{\tau}_{\boldsymbol{k}}$ can arise only if the sign of the second-order coefficient of this mode changes to negative. Upon increasing incompatibility $L \chi$ (lowering temperature), the disordered, homogeneously mixed state is destroyed by that mode whose coefficient changes sign first (see the model free-energy function in fig. 3.3). By inspection of the second-order coefficients in eqs. (3.25) and (3.32), the global second-order structure function, eq. (3.23), determines 

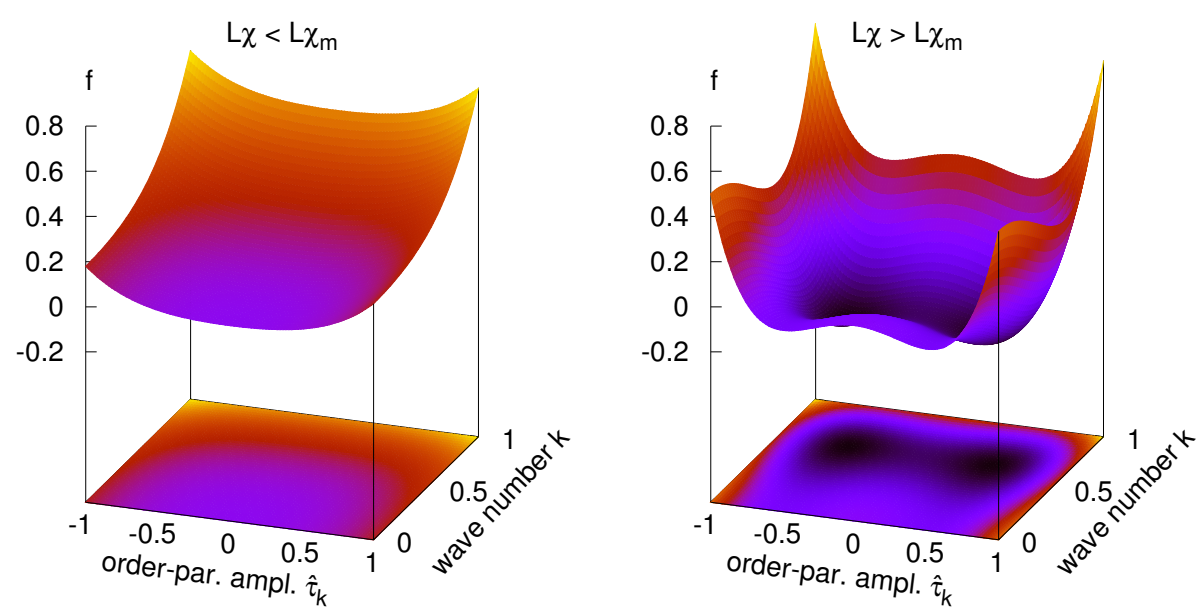

Figure 3.3: Single-harmonic contributions to a sample free-energy function $f$, pointing to a disordered state at low incompatibilities $L \chi$ (left panel), and to a microstructured morphology as the first instability on increasing $L \chi$ (right panel), indicated by minima at nonzero orderparameter amplitudes and finite wave number.

via its maximum, $\max _{k} S\left(k^{2}\right)$, the critical wave number $k_{0}$ of the instability, viz.

$$
k_{0}=\underset{k}{\operatorname{argmax}} S\left(k^{2}\right) .
$$

Accordingly, the instability occurs at the critical incompatibility

$$
L \chi_{0}=\frac{2 L^{2}}{S\left(k_{0}^{2}\right)}
$$

Given the critical wave number $k_{0}$, we have to locate the minima of the free-energy functional on the axis $\hat{\tau}_{k_{\mathrm{m}}}$, viz., the order-parameter amplitude of the coexisting $A$ and $B$-rich domains (or macroscopic phases, if $k_{0}=0$ ), as sketched in fig. 3.3. This minimization will be performed in sections 3.4 and 3.5 .

The form of the free-energy functional, eq. 3.25 or eq. 3.32 , suggests that $L \chi$, the product of incompatibility $\chi$ and degree of polymerization $L$, is the main control parameter to determine phase separation. This is seen most easily for the symmetric mixture of $A$ and $B$ homopolymers, in which the moment $m_{2}=S(0) / L^{2}$ equals one for any $L$ and macroscopic phase separation occurs at a fixed value $L \chi_{0}=2 / m_{2}$. For a random distribution of $Q$-block copolymers in turn, the function $S\left(k^{2}\right) / L^{2}$ is characteristic of the average type correlation on a chain, $S\left(k^{2}\right) / L^{2} \leq 1$ for any $L$. Especially, given the sequence distribution and the structure of the instability, there is still a scaling of the critical incompatibility with $L$ or with $M$, the number of statistical segments per block. Varying the sequence distribution, $M$ is found to be decisive for the critical wave number of the instability as a function of block-type correlation, as detailed below. 


\subsection{Global structure function and multicritical point}

As we have seen in sec. 3.2, the second-order coefficient of the functional eq. (3.25) or eq. (3.32) sets the limit of stability of the disordered, homogeneously mixed state of a symmetric sequence distribution. More precisely, the maximum of the second-order structure function $S\left(k^{2}\right)$ determines the incompatibility at which a phase separation takes place, and the maximum position indicates the wave number of the $A$ - $B$ modulation to emerge. In view of the significance of $S\left(k^{2}\right)$ for the instability, we briefly digress to establish its connection with a measurable observable of the disordered melt. According to eqs. (3.23) and (C.8), the function $S\left(k^{2}\right)$ is a Fourier-transformed, intra-chain densitydensity correlation sensitive to segment types, averaged over all copolymer sequences in the melt. Within saddle-point approximation, the second-order coefficient of our functional defines the $A$ excess density-density correlation of the entire, homogeneous melt with (weakly) incompatible $A$ and $B$ segments as well. The $A$ excess density-density correlation in turn is proportional to the scattering intensity, accessible in experiments in which one segment type can be tagged [43], or generally, if $A$ and $B$ segments have different scattering amplitudes.

From the quadratic term of our effective Hamiltonian, cf. eq. 3.18 ) or eq. (3.25), we obtain the variance of the interaction field $\hat{\sigma}$ at the saddle-point level as

$$
\left\langle\hat{\sigma}_{\boldsymbol{k}} \hat{\sigma}_{-\boldsymbol{k}}\right\rangle_{\mathrm{cov}}:=\left\langle\hat{\sigma}_{\boldsymbol{k}} \hat{\sigma}_{-\boldsymbol{k}}\right\rangle_{N \hat{h}}-\left\langle\hat{\sigma}_{\boldsymbol{k}}\right\rangle_{N \hat{h}}\left\langle\hat{\sigma}_{-\boldsymbol{k}}\right\rangle_{N \hat{h}}=\frac{2 \varrho_{0}^{2}}{N L \chi}\left(1-L \chi \frac{S\left(k^{2}\right)}{2 L^{2}}\right)^{-1} .
$$

By observing the relation eq. (3.29), we can derive the variance of the collective A excess density at wave vector $\boldsymbol{k}$, and hence the scattering function $\Sigma\left(k^{2}\right)$. In effect, we have

$$
\Sigma\left(k^{2}\right) \propto\left\langle\sigma_{\boldsymbol{k}} \sigma_{-\boldsymbol{k}}\right\rangle_{\mathrm{cov}} \propto\left(\frac{1}{S\left(k^{2}\right)}-\frac{N L \chi}{\varrho_{0}^{2}}\right)^{-1} .
$$

An equivalent expression has been derived in random phase approximation (RPA), in the linear response regime [44, 76, 18, see appendices A.2.2 and A.3. Fitting of experimental scattering data to a theoretical curve of the form of eq. (3.36) may be used to obtain parameters of a block copolymer melt, e.g., the effective incompatibility [54].

\subsubsection{Multicriticality for symmetric Markovian sequence distributions}

For Markovian sequence distributions with zero global $A$ excess, solely the block-type correlation parameter $\lambda$ decides whether the maximum position of $S\left(k^{2}\right)$ is located at zero or at a finite wave number. In the former case, the disordered state becomes unstable with respect to macroscopic phase separation, in the latter case to microphase separation [76, 41, 42]. Upon decreasing $\lambda$, the maximum position of $S\left(k^{2}\right)$ attains a nonzero value at a critical correlation $\lambda_{c}(M)$, depending on the number $M$ of segments per block. The corresponding point in the $\lambda$ - $\chi$ plane, at which the lines of macroscopic, respectively lamellar phase separations meet (recall the sketch in fig. 1.7), is termed a multicritical point, since the parameter regions of the disordered state, macroscopic phase separation, lamellae, and of the conjectured three-phase coexistence must meet at this point, too. 
For a $\lambda$-distribution of $Q$-block copolymers with $M$ segments per block, $S\left(k^{2}\right)$ can be calculated from the probabilities of all type combinations of two segments with a given intra-chain distance (in blocks) using the transition matrix $\hat{M}$ (cf. eqs. B.13) and (C.14) in the appendix):

$$
S\left(k^{2}\right)=Q D\left(M, k^{2}\right)+\frac{2 \lambda \mathrm{e}^{-M k^{2}} \sinh ^{2}\left(\frac{M k^{2}}{2}\right)}{\left(1-\lambda \mathrm{e}^{-M k^{2}}\right) \sinh ^{2}\left(\frac{k^{2}}{2}\right)}\left\{Q-\frac{1-\left(\lambda \mathrm{e}^{-M k^{2}}\right)^{Q}}{1-\lambda \mathrm{e}^{-M k^{2}}}\right\}
$$

with the dimensionless wave number $k^{2}:=b^{2} \tilde{k}^{2} /(2 d)$ and $\tilde{k}$ the physical wave number. The discrete Debye function $D\left(L, k^{2}\right)$ is given in eq. (C.6). In order to assign in the subsequent fractionation scheme to each $\lambda$ the morphology of the disordered state's global instability, we distinguish two parts of the line of instabilities:

- For $\lambda>\lambda_{c}$ (e.g., $\lambda_{c}=-1 / 3$ for $Q=M=3$ ), the maximum of $S\left(k^{2}\right)$ is at $k_{0}=0$, so, according to eq. (3.33), the instability of the disordered state in a $\lambda$-distribution with $\lambda>\lambda_{c}$ is toward two macroscopic, homogeneous, $A$ - and $B$-rich phases. This macroscopic phase separation sets in at (see eqs. (3.34) and (3.31)

$$
L \chi_{0}(\lambda)=\frac{2}{m_{2}\left(\left\{p_{\nu}(\lambda)\right\}\right.} .
$$

- For $\lambda<\lambda_{c}$, the maximum of $S\left(k^{2}\right)$ at $k_{0}>0$ indicates that the instability of the disordered state is with respect to a lamellar phase.

For continuous Gaussian block copolymers (parameterized by an arc-length instead of an integer segment index) with unaltered chain radius of gyration, the structure functions are computed in the combined limit $M \rightarrow \infty, b^{2} \rightarrow 0, M b^{2}=$ const., abbreviated as $\lim _{M \rightarrow \infty}$, preserving the given finite number of blocks $(Q=3$ for our sample system of random triblocks) and the rms end-to-end distance $R_{\text {block }}=\sqrt{M} b$. In this case, the wave number is conveniently rescaled with $R_{\text {block}}$. For a $\lambda$-distribution of continuous $Q$-block copolymers, the global second-order structure function is

$$
\begin{aligned}
s\left(k^{2}\right) & :=\lim _{M \rightarrow \infty} S\left(k^{2} / M\right) / M^{2} \\
& =Q d\left(1, k^{2}\right)+\frac{2 \lambda \mathrm{e}^{-k^{2}} \sinh ^{2}\left(\frac{k^{2}}{2}\right)}{\left(1-\lambda \mathrm{e}^{-k^{2}}\right) k^{4} / 4}\left\{Q-\frac{1-\left(\lambda \mathrm{e}^{-k^{2}}\right)^{Q}}{1-\lambda \mathrm{e}^{-k^{2}}}\right\},
\end{aligned}
$$

now with $k^{2}:=R_{\text {block }}^{2} \tilde{k}^{2} /(2 d)$, and the continuous Debye function (cf. eq. C.7)

$$
d\left(\ell, k^{2}\right):=\frac{\mathrm{e}^{-\ell k^{2}}-1+\ell k^{2}}{k^{4} / 2}
$$

\subsubsection{Multicriticality for triblock copolymers}

Now, we discuss the line of instabilities of the disordered state toward a global, ordered state specifically for the case of Markovian, symmetric triblock copolymers, $Q=3$. In 
this system, the change of the peak position of the global structure function at the critical correlation $\lambda_{c}(M)$ induces the following transition from macroscopic to lamellar phase separation:

a) For $M \leq 6$, the maximum of $S\left(k^{2}\right)$ is located at $k_{0}=0$ for all $\lambda \geq \lambda_{c}(M)$ and grows continuously from $k_{0}=0$ when $\lambda$ falls below $\lambda_{c}(M)$. Thus, the critical value of the correlation $\lambda_{c}(M)$ is reached when the second derivative of $S\left(k^{2}\right)$ at $k=0$ changes sign:

$$
\lambda_{c}(M)=-\frac{1}{2}\left(1-\frac{1}{M}\right), \quad M \leq 6 .
$$

b) For $M>6$, however, a second maximum of $S\left(k^{2}\right)$ at $k>0$ evolves already for $\lambda>\lambda_{c}(M)$. Now, the critical value $\lambda_{c}$ of $\lambda$ is the one at which the second maximum (pointing to a metastable lamellar phase) attains a higher value than the one at $k=0$, and thus, $\lambda_{c}$ is accessible numerically only.

Continuous triblocks realize case $b$ ), consistent with the case of triblocks with $M>6$ discrete segments. The wave number of the global ordered (lamellar) state, $k_{0}(\lambda)$, jumps discontinuously to zero as $\lambda$ approaches $\lambda_{c}=-0.464$ from below. The lamellar phase persists as a metastable state for $\lambda>\lambda_{c}$, as well as macroscopic phase separation (two homogenous phases) for $\lambda<\lambda_{c}$. Remarkably, we find this discontinuity of the global wave number precisely for the broader class of triblock copolymers with $M>6$ segments per block, whereas the literature on copolymer mixtures seems to report only the behavior a) (see, e.g., [18, 70]), associated with a Lifshitz point [61].

\subsubsection{Structure functions for individual triblock sequences}

In order to address subsystems with sequence distributions different from the global ( $\lambda$-defined $)$ distribution in the next chapter, we will have to decompose the global second-order structure function eq. 3.23$)$ into the contributions $S_{\nu}\left(k^{2}\right)$ of individual sequences. Here, we provide our results for the sequence-specific $S_{\nu}\left(k^{2}\right)$ of random symmetric triblock copolymers, $Q=3$, the sample system throughout this work. This system features six different sequences, which fall into only three distinct sequence classes defined by the topology and number of unfavorable intra-chain $A$ - $B$ contacts (cf. appendix B.1), and which we index as follows:

1: JJJ; homopolymers; all block-types equal,

$2: J K K$; two adjacent blocks of equal type,

$3: J K J$; strictly alternating block-types,

$J, K \in\{A, B\}, J \neq K$. One class comprises a pair of sequences, such as $A A B$ and $B B A$, related by permutation of $A$ and $B$, for which the structure functions and moments appearing in the functional eq. (3.25), respectively (3.32), relevant for the $A \leftrightharpoons B$ exchange-symmetric case, are identical. For a $\lambda$-distribution of random triblocks, the probabilities of these sequence classes are:

$$
p_{1}(\lambda)=\frac{(1+\lambda)^{2}}{4}, \quad p_{2}(\lambda)=\frac{1-\lambda^{2}}{2}, \text { and } p_{3}(\lambda)=\frac{(1-\lambda)^{2}}{4} .
$$


The individual structure functions eq. 3.20 for each of the sequence classes defined in eq. (3.42) are

$$
\begin{aligned}
& S_{1}\left(k^{2}\right)=D\left(3 M, k^{2}\right)=\frac{3 M\left(1+\mathrm{e}^{-k^{2}}\right)}{1-\mathrm{e}^{-k^{2}}}-\frac{2 \mathrm{e}^{-k^{2}}\left(1-\mathrm{e}^{-3 M k^{2}}\right)}{\left(1-\mathrm{e}^{-k^{2}}\right)^{2}}, \\
& S_{2}\left(k^{2}\right)=-D\left(3 M, k^{2}\right)+2 D\left(2 M, k^{2}\right)+2 D\left(M, k^{2}\right) \\
& S_{3}\left(k^{2}\right)=D\left(3 M, k^{2}\right)-4 D\left(2 M, k^{2}\right)+8 D\left(M, k^{2}\right)
\end{aligned}
$$

(as an example, $S_{2}\left(k^{2}\right)$ is derived in appendix C.3). While the maximum of $S_{1}\left(k^{2}\right)$ is located at $k=0$, the maxima of $S_{2}\left(k^{2}\right)$ and $S_{3}\left(k^{2}\right)$ are at $k>0$, cf. fig. 3.4 , due to the characteristic correlation length of identical segments within copolymers of the sequence classes 2 and 3 .
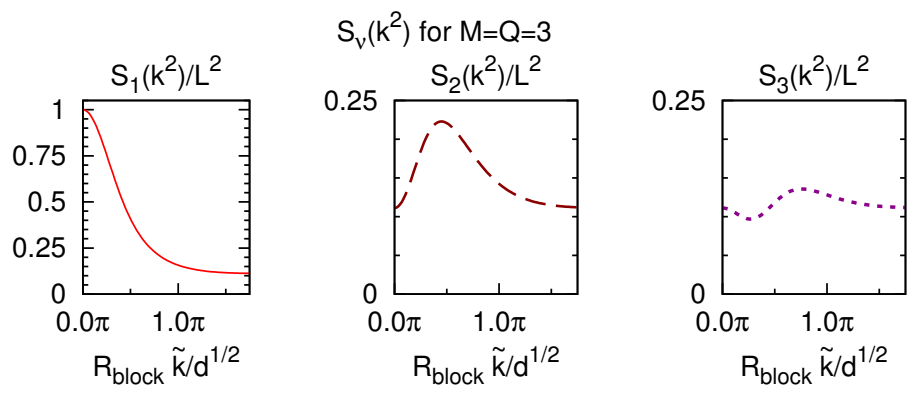

Figure 3.4: Structure functions $S_{\nu}\left(k^{2}\right)$ for the triblock sequence classes defined in eq. 3.42 , for $M=3$ segments per block.

The continuous-chain versions can be constructed in analogy with the continuous Debye functions, e.g., the structure function eq. (3.44b) for sequence class 2 is

$$
s_{2}\left(k^{2}\right):=\lim _{M \rightarrow \infty} S_{2}\left(k^{2} / M\right) / M^{2}=-d\left(3, k^{2}\right)+2 d\left(2, k^{2}\right)+2 d\left(1, k^{2}\right),
$$

again with $k^{2}:=R_{\text {block }}^{2} \tilde{k}^{2} /(2 d)$. In the following, $S_{\nu}\left(k^{2}\right)$ or $S\left(k^{2}\right)$ refer to the structure functions for chains with discrete segments; the number $M$ of segments per block is usually not listed as an argument separately. The continuous-chain versions are denoted with $s_{\nu}\left(k^{2}\right), s\left(k^{2}\right)$, etc. In order to obtain the continuous-chain limit of expressions that involve second-order structure functions, the discrete versions, $S_{\nu}\left(k^{2}\right)$, are to be replaced by $M^{2} s_{\nu}\left(M k^{2}\right)$. For a quantitative comparison of wave numbers, note the different scales, viz. segment, respectively block size, for discrete, respectively continuous chains, eqs. 3.37) and 3.39).

For practical reasons, the explicit calculation of sequence-specific structure functions is limited to distributions with a comparatively small number of different sequences, i.e., to a small number $Q$ of blocks, since the number of sequences grows exponentially with $Q$, cf. eq. (2.6). Instead of six sequences in the case of random triblock copolymers, already 10 different sequences are present in a random quadblock copolymer melt, and 20 in a random pentablock copolymer melt.

\subsection{Lamellar phase separation}

A spatially periodic structure of $A$ - and $B$-rich domains destabilizes the disordered state, if the critical wavenumber $k_{0}$, cf. eq. (3.33), is nonzero. This nonzero wave number of the 
instability is called $k_{\mathrm{m}}$. In a Markovian ( $\lambda$-defined) sequence distribution, $p_{\nu}=p_{\nu}(\lambda)$, this is the case only for block-type correlations $\lambda<\lambda_{c}$, see section 3.3.1. In a subsystem with a different set of sequence probabilities, structured phase separation may occur also for $\lambda>\lambda_{c}$. For $A \leftrightharpoons B$ exchange-symmetric block copolymer melts, the structured phase consistent with the symmetry has a single wave-vector direction only, since plane interfaces between $A$ - and $B$-rich domains, i.e., lamellae, yield the lowest free energy [76, 94, 125]. Moreover, the emerging lamellae can be assumed to be dominated by a single wave number also on depart from the transition (but not too far), i.e., for $L \chi>L \chi_{0}$. Consequently, the simplest parameterization for the order-parameter field $\hat{\tau}$ is a single mode with an arbitrary, but fixed unit normal $\boldsymbol{n}$ of the domain interfaces. The parameters are the wave number $k_{\mathrm{m}}=k_{0}>0$, the wave vector being $\boldsymbol{k}=k_{\mathrm{m}} \boldsymbol{n}$, and the amplitude $\hat{\tau}_{k_{\mathrm{m}}}$ :

$$
\hat{\tau}_{\boldsymbol{k}}=\hat{\tau}_{k_{\mathrm{m}}}\left(\delta_{\boldsymbol{k}, k_{\mathrm{m}} \boldsymbol{n}}+\delta_{\boldsymbol{k},-k_{\mathrm{m}} \boldsymbol{n}}\right)
$$

Evaluating the free-energy functional eq. (3.25) or eq. (3.32) for a structured phase rests on small order-parameter amplitudes, i.e., weak $A-B$ separation, which is reasonable close to the phase transition (often called order-disorder transition in the case of a structured phase).

\subsubsection{Simplified lamellar free energy}

Inserting eq. (3.46) into the simplified free-energy functional eq. (3.32) yields

$$
f\left(k_{\mathrm{m}}, \hat{\tau}_{k_{\mathrm{m}}}\right)=\sum_{\nu} p_{\nu} \ln p_{\nu}+\frac{(L \chi)^{2}}{2 \varrho_{0}^{2}}\left(\frac{1}{L \chi}-\frac{S\left(k_{\mathrm{m}}^{2}\right)}{2 L^{2}}\right) \hat{\tau}_{k_{\mathrm{m}}}^{2}+\frac{(L \chi)^{4}}{64 \varrho_{0}^{4}}\left(m_{2}^{2}+m_{4}\right) \hat{\tau}_{k_{\mathrm{m}}}^{4},
$$

an expression valid only for incompatibilities $L \chi$ above the critical $L \chi_{0}$ for the lamellar state, cf. eq. (3.34). The stability of eq. (3.47) is guaranteed by the fourth-order coefficient, which is nonnegative for an arbitrary sequence distribution. Since the fourthorder coefficient does not depend on $k$ in the simplified description, the lamellar profile continues to display the initial wave number $k_{\mathrm{m}}$ also at $L \chi>L \chi_{0}$. The type correlation length of the sequence (distribution) is encoded solely in the second-order structure function $S\left(k^{2}\right)$. Subsequent minimization of the free-energy functional eq. 3.47) with respect to the amplitude $\hat{\tau}_{k_{\mathrm{m}}}$ gives the optimal amplitude $\hat{\tau}_{\mathrm{m}}$,

$$
\frac{\hat{\tau}_{\mathrm{m}}^{2}}{\varrho_{0}^{2}}=\frac{16}{(L \chi)^{2}} \frac{\frac{S\left(k_{\mathrm{m}}^{2}\right)}{2 L^{2}}-\frac{1}{L \chi}}{m_{2}^{2}+m_{4}} \text { with } k_{\mathrm{m}}:=\underset{k>0}{\operatorname{argmax}} S\left(k^{2}\right) .
$$

With this amplitude, the free-energy density of the lamellar phase is

$$
f_{\mathrm{m}}=\sum_{\nu} p_{\nu} \ln p_{\nu}-\frac{\left(\frac{S\left(k_{\mathrm{m}}^{2}\right)}{L^{2}}-\frac{2}{L \chi}\right)^{2}}{m_{2}^{2}+m_{4}}, L \chi \geq L \chi_{0}:=\frac{2 L^{2}}{S\left(k_{\mathrm{m}}^{2}\right)} .
$$

In preparation for the analysis of fractionation, which allows for arbitrary symmetric sequence distributions, we specify the dependence on the sequence probabilities and 
rewrite $f_{\mathrm{m}}$ from eq. $(3.49)$ in the form

$$
f_{\mathrm{m}}=\sum_{\nu} p_{\nu} \ln p_{\nu}-\frac{\left(\frac{\sum_{\nu} p_{\nu} S_{\nu}\left(k_{\mathrm{m}}^{2}\right)}{L^{2}}-\frac{2}{L \chi}\right)^{2}}{\left(\sum_{\nu} p_{\nu} m_{2, \nu}\right)^{2}+\sum_{\nu} p_{\nu} m_{4, \nu}}, k_{\mathrm{m}}:=\underset{k>0}{\operatorname{argmax}} \sum_{\nu} p_{\nu} S_{\nu}\left(k^{2}\right),
$$

again for $L \chi \geq L \chi_{0}$. In this form, $k_{\mathrm{m}}$ is seen to depend on the sequence concentrations, $k_{\mathrm{m}}=k_{\mathrm{m}}\left(\left\{p_{\nu}\right\}\right)$; thereby, $S\left(k_{\mathrm{m}}^{2}\right)$ and $L \chi_{0}$ become functions of the $p_{\nu}$ as well. The first phase diagrams in chapter 5 are based on the lamellar free-energy density eq. (3.50).

\subsubsection{Free energy with wave-number dependence of fourth-order coefficients}

In an effort to explore the possible parameters of a lamellar phase in more detail, we restore the wave-number dependence in the fourth-oder coefficients of eq. (3.25). Inserting into eq. (3.25) a single-harmonic profile analogous to eq. (3.46), but with a yet undetermined wave number $k$, we obtain

$$
\begin{aligned}
f\left(k, \hat{\tau}_{k}\right)= & \sum_{\nu} p_{\nu} \ln p_{\nu}+\frac{(L \chi)^{2}}{2 \varrho_{0}^{2}}\left(\frac{1}{L \chi}-\frac{S\left(k^{2}\right)}{2 L^{2}}\right) \hat{\tau}_{k}^{2} \\
& +\frac{(L \chi)^{4}}{64 \varrho_{0}^{4}}\left(\frac{\left(S^{(\alpha)}(k \boldsymbol{n}, k \boldsymbol{n})\right)^{2}}{L^{4} D\left(L, 4 k^{2}\right)}-\frac{S^{(\beta)}(k \boldsymbol{n}, k \boldsymbol{n},-k \boldsymbol{n})}{L^{4}}+2 \frac{S^{(\gamma)}\left(k^{2}, k^{2}\right)}{L^{4}}\right) \hat{\tau}_{k}^{4} .
\end{aligned}
$$

(Note that the higher-order structure functions, defined in eqs. (3.22), (3.26), (3.27), and computed in appendix C.5.2, are independent of the unit normal $\boldsymbol{n}$.) Again, the instability of the disordered state toward the lamellar phase (of zero amplitude) occurs at $L \chi_{0}$, cf. eq. (3.34), with the critical wave number $k_{\mathrm{m}}=\operatorname{argmax} S\left(k^{2}\right)>0$. For $L \chi$ above $L \chi_{0}$, however, the wave number $k_{\text {opt }}$ that maximizes the lamellar amplitude $\hat{\tau}_{k}$,

$$
\frac{\hat{\tau}_{k}^{2}}{\varrho_{0}^{2}}=\frac{16}{(L \chi)^{2}} \frac{S\left(k^{2}\right) /\left(2 L^{2}\right)-1 /(L \chi)}{\frac{\left(S^{(\alpha)}(k \boldsymbol{n}, k \boldsymbol{n})\right)^{2}}{L^{4} D\left(L, 4 k^{2}\right)}-\frac{S^{(\beta)}(k \boldsymbol{n}, k \boldsymbol{n},-k \boldsymbol{n})}{L^{4}}+2 \frac{S^{(\gamma)}\left(k^{2}, k^{2}\right)}{L^{4}}}, L \chi \geq L \chi_{0},
$$

and thus minimizes the free-energy density, is no longer confined to $k_{\mathrm{m}}$, due to the dependence of the denominator in eq. 3.52 on $k$. Instead, $k_{\text {opt }}$ must be searched at each $L \chi$ among all $k$ in a constrained neighborhood of $k_{\mathrm{m}}$,

$$
\begin{gathered}
k_{\mathrm{opt}}:=\underset{k \in \mathcal{U}\left(k_{\mathrm{m}}\right)}{\operatorname{argmax}} \frac{S\left(k^{2}\right) /\left(2 L^{2}\right)-1 /(L \chi)}{\frac{\left(S^{(\alpha)}(k \boldsymbol{n}, k \boldsymbol{n})\right)^{2}}{L^{4} D\left(L, 4 k^{2}\right)}-\frac{S^{(\beta)}(k \boldsymbol{n}, k \boldsymbol{n},-k \boldsymbol{n})}{L^{4}}+2 \frac{S^{(\gamma)}\left(k^{2}, k^{2}\right)}{L^{4}},} \\
\mathcal{U}\left(k_{\mathrm{m}}\right):=\left\{k: L \chi \geq 2 L^{2} / S\left(k^{2}\right) \geq 2 L^{2} / S\left(k_{\mathrm{m}}^{2}\right)\right\} .
\end{gathered}
$$

Hence, the optimization of the amplitude results in a change of the lamellar wave number with increasing $L \chi$, such that $k_{\mathrm{opt}}=k_{\mathrm{opt}}\left(L \chi ;\left\{p_{\nu}\right\}\right)$. (In the simplified lamellar freeenergy density, only the amplitude changes on increasing $L \chi$. .) In evaluating eq. 3.25) 
and keeping the contribution of one dominant wave number, we again address values of $L \chi$ not too far from the lamellar instability. Inserting the optimal amplitude determined by $k_{\text {opt }}$ into eq. (3.51), we arrive at the lamellar free-energy density

$$
\begin{aligned}
f_{\mathrm{m}}= & \sum_{\nu} p_{\nu} \ln p_{\nu} \\
& -\frac{\left(S\left(k_{\mathrm{opt}}^{2}\right)-2 L / \chi\right)^{2}}{\frac{\left(S^{(\alpha)}\left(k_{\mathrm{opt}} \boldsymbol{n}, k_{\mathrm{opt}} \boldsymbol{n}\right)\right)^{2}}{D\left(L, 4 k_{\mathrm{opt}}^{2}\right)}-S^{(\beta)}\left(k_{\mathrm{opt}} \boldsymbol{n}, k_{\mathrm{opt}} \boldsymbol{n},-k_{\mathrm{opt}} \boldsymbol{n}\right)+2 S^{(\gamma)}\left(k_{\mathrm{opt}}^{2}, k_{\mathrm{opt}}^{2}\right)},
\end{aligned}
$$

for $L \chi>2 L^{2} / S\left(k_{\text {opt }}^{2}\right)>L \chi_{0}$.

\subsection{Macroscopic phase separation}

If the maximum of the second-order structure function is located at zero wave number, the free-energy functional eq. (3.32) indicates an instability of the disordered state toward the separation into two macroscopic $A$-, respectively $B$-rich phases, each internally homogeneous in $A$ content. The apparent conflict with restricting the summations in eq. (3.32) to order-parameter modulations, with $\boldsymbol{k} \neq \mathbf{0}$, stems from the fact that this functional addresses the free energy of a structured, one-phase state with an infinite number of $A$ - and $B$-rich domains. Two or more macroscopic phases cannot be covered by a structured, single-phase ansatz in the limit $\boldsymbol{k} \rightarrow \mathbf{0}$, except at zero order-parameter amplitude. However, the incompatibility $L \chi_{0}$ of the instability toward two homogeneous phases can be read off from the second-order terms of eqs. (3.25) or (3.32), cf. eq. (3.34):

$$
L \chi_{0}:=\frac{2}{m_{2}}=\frac{2}{\sum_{\nu} p_{\nu} m_{2, \nu}}
$$

(at higher incompatibilities, separations into more than two homogeneous phases may arise, cf., e.g., [91]).

In order to calculate the free-energy density in macroscopic phase separation, we have to derive a real-space version of the free-energy functional which allows for a correct ansatz of multiple homogeneous phases before taking the thermodynamic limit. 4 Fortunately, by redefining our set of variables for the cases in which eq. 3.32 indicates macroscopic phase separation, we can derive for the free energy of coexisting homogeneous phases a closed expression that is not limited to small order-parameter amplitudes or weak $A-B$ segregation. Such an expression is preferable to the expansion for our analysis of the transition from the macroscopically separated, two-phase state to a three-phase coexistence. The latter transition can in general occur at any finite amplitude of the two homogeneous phases, at an incompatibility considerably larger than that of the transition between disordered and macroscopically phase-separated state, see fig. 5.1 below. Given that, in the mean-field approach to homogeneous phases, a copolymer chain can only experience and contribute to interaction fields uniform

\footnotetext{
${ }^{4}$ Taking the limit $k_{\mathrm{m}} \rightarrow 0$ in the free energy of one lamellar phase, eq. 3.49 , amounts to interchanging thermodynamic and long-wavelength limit and fails to reproduce the expansion of eq. 3.74 below.
} 
within one phase, we may replace a copolymer's set of segment types and positions by a single position vector $\boldsymbol{r}_{j}$ and by an $A$ excess $\tilde{q}_{j}$ equal to the average over all segments on that copolymer $j$ (cf. fig. 3.5). Effectively, the internal $A-B$ sequence and conformational
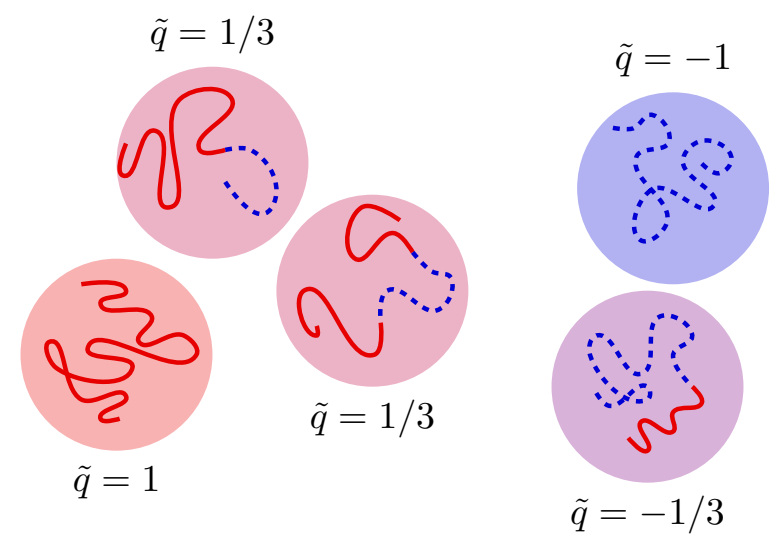

Figure 3.5: Reduced degrees of freedom of a "crushed" triblock copolymer: center-of-mass position and overall A excess $\tilde{q}$.

degrees of freedom, irrelevant in the presence of uniform fields, are pre-averaged in this coarse-grained "crushed polymer" picture.

In a distribution of $Q$-block copolymers, we distinguish $(Q+1)$ components by their composition or $A$-excess,

$$
\tilde{q}_{c}:=\frac{2 c-Q}{Q}=-\tilde{q}_{Q-c}, \quad c \in\{0,1, \ldots, Q\},
$$

where the component index $c$ is the number of $A$-blocks. All copolymers with the same number of $A$-blocks contribute as structureless particles to one component density

$$
\varrho_{c}(\boldsymbol{r})=\frac{L}{V} \sum_{j=1}^{N} \delta_{\tilde{q}_{j}, \tilde{q}_{c}} \delta\left(\boldsymbol{r}-\boldsymbol{r}_{j}\right), \quad c \in\{0,1, \ldots, Q\},
$$

(the factor $L$ accounts for the original number of segments, in order to preserve the average density $N L / V)$. The overall number of copolymers of component $c$ is $N \tilde{p}_{c}$. With these component densities, the total segment and $A$ excess densities are

$$
\varrho(\boldsymbol{r})=\sum_{c} \varrho_{c}(\boldsymbol{r}), \quad \sigma(\boldsymbol{r})=\sum_{c} \tilde{q}_{c} \varrho_{c}(\boldsymbol{r}),
$$

and the canonical partition function to calculate is

$$
\mathcal{Z}=\frac{1}{\Omega} \prod_{j=1}^{N}\left(\int \frac{\mathrm{d}^{d} r_{j}}{V}\right) \exp \left\{\frac{V^{2}}{4 \varrho_{0}} \int \mathrm{d}^{d} r\left(\chi(\sigma(\boldsymbol{r}))^{2}-\kappa(\varrho(\boldsymbol{r}))^{2}\right)\right\} .
$$

Again introducing interaction fields, the derivation of the free-energy functional is performed analogously as in sec. 3.1 for structured, ordered phases, but is more straightforward, since the averages over chain conformations are obsolete. Application of the (pointwise) Hubbard-Stratonovich transformation with interaction fields $\hat{\sigma}, \varrho \varrho$ results in the effective Hamiltonian per copolymer

$$
\tilde{h}=\frac{V^{2}}{4 N \varrho_{0}} \int \mathrm{d}^{d} x\left(\chi(\hat{\sigma}(\boldsymbol{x}))^{2}+\kappa(\hat{\varrho}(\boldsymbol{x}))^{2}\right)-\sum_{c} \tilde{p}_{c} \ln \tilde{z}_{c}+\sum_{\nu} p_{\nu} \ln p_{\nu}
$$


analogous to eq. (3.15). In this expression, the single-component partition functions are

$$
\tilde{z}_{c}:=\frac{1}{V} \int \mathrm{d}^{d} x \exp \left\{\frac{L}{2 \varrho_{0}}\left(\chi \tilde{q}_{c} \hat{\sigma}(\boldsymbol{x})+i \kappa \hat{\varrho}(\boldsymbol{x})\right)\right\} .
$$

In eq. (3.60a), the terms $p_{\nu} \ln p_{\nu}$ reflect the combinatorial factor $\Omega$.

\subsubsection{Multiple homogeneous phases}

The general ansatz of $H \leq(Q+1)$ coexisting macroscopic phases, each with uniform values of the fields,

$$
\left.\begin{array}{l}
V \hat{\varrho}(\boldsymbol{x})=\hat{\varrho}^{(h)}=\sum_{c} \hat{\varrho}_{c}^{(h)}, \\
V \hat{\sigma}(\boldsymbol{x})=\hat{\sigma}^{(h)}=\sum_{c} \tilde{q}_{c} \hat{\varrho}_{c}^{(h)},
\end{array}\right\} \boldsymbol{x} \in V^{(h)}, h \in\{1, \ldots, H\},
$$

and with volume fractions $v^{(h)}:=\left|V^{(h)}\right| / V$, yields

$$
\begin{aligned}
& \tilde{h}=\frac{L \chi}{4 \varrho_{0}^{2}} \sum_{h} v^{(h)}\left(\hat{\sigma}^{(h)}\right)^{2}+\frac{L \kappa}{4 \varrho_{0}^{2}} \sum_{h} v^{(h)}\left(\hat{\varrho}^{(h)}\right)^{2}-\sum_{c} \tilde{p}_{c} \ln \tilde{z}_{c}+\sum_{\nu} p_{\nu} \ln p_{\nu}, \\
& \tilde{z}_{c}=\sum_{h} v^{(h)} \exp \left\{\frac{L\left(\chi \tilde{q}_{c} \hat{\sigma}^{(h)}+i \kappa \hat{\varrho}^{(h)}\right)}{2 \varrho_{0}}\right\}
\end{aligned}
$$

For convenience, the volume fraction of one phase, labeled $h_{0}$, can be eliminated immediately in favor of the volume fractions of the $(H-1)$ remaining phases. Now, the set of saddle-point equations $0=\partial \tilde{h} / \partial \hat{\sigma}^{(h)}, 0=\partial \tilde{h} / \partial \hat{\varrho}^{(h)}, 0=\partial \tilde{h} / \partial v^{(h)}$, becomes

$$
\begin{aligned}
\frac{\hat{\tau}^{(h)}}{\varrho_{0}}= & \sum_{c} \tilde{q}_{c} \frac{\tilde{p}_{c}}{\tilde{z}_{c}} \exp \left\{\frac{L\left(\chi \tilde{q}_{c} \hat{\tau}^{(h)}+i \kappa \hat{\omega}^{(h)}\right)}{2 \varrho_{0}}\right\} \\
\frac{\hat{\omega}^{(h)}}{\varrho_{0}}= & i \sum_{c} \frac{\tilde{p}_{c}}{\tilde{z}_{c}} \exp \left\{\frac{L\left(\chi \tilde{q}_{c} \hat{\tau}^{(h)}+i \kappa \hat{\omega}^{(h)}\right)}{2 \varrho_{0}}\right\} \\
0= & \frac{L \chi}{4 \varrho_{0}^{2}}\left(\left(\hat{\tau}^{(h)}\right)^{2}-\left(\hat{\tau}^{\left(h_{0}\right)}\right)^{2}\right)+\frac{L \kappa}{4 \varrho_{0}^{2}}\left(\left(\hat{\omega}^{(h)}\right)^{2}-\left(\hat{\omega}^{\left(h_{0}\right)}\right)^{2}\right) \\
& -\sum_{c} \frac{\tilde{p}_{c}}{\tilde{z}_{c}}\left(\exp \left\{\frac{L\left(\chi \tilde{q}_{c} \hat{\tau}^{(h)}+i \kappa \hat{\omega}^{(h)}\right)}{2 \varrho_{0}}\right\}-\exp \left\{\frac{L\left(\chi \tilde{q}_{c} \hat{\tau}^{\left(h_{0}\right)}+i \kappa \hat{\omega}^{\left(h_{0}\right)}\right)}{2 \varrho_{0}}\right\}\right)
\end{aligned}
$$

where again we denoted the saddle-point values of $\hat{\sigma}^{(h)}, \hat{\varrho}^{(h)}$ as $\hat{\tau}^{(h)}, \hat{\omega}^{(h)}$. Multiplication of eqs. 3.63b or 3.63a with their volume fractions $v^{(h)}$ and summation over $h$ shows that the conditions of fixed global values of density and of $A$ excess,

$$
\sum_{h} v^{(h)} \hat{\tau}^{(h)}=\sum_{c} \tilde{p}_{c} \tilde{q}_{c}=q, \quad \sum_{h} v^{(h)} \hat{\omega}^{(h)}=i \varrho_{0},
$$

are fulfilled (note that $\hat{\omega}$ is related to the saddle point of the density via $\hat{\omega}=i\langle\varrho\rangle_{\mathcal{H}}$ ). 
By inserting eqs. 3.63b into eqs. 3.63c), we can solve for the difference in $\kappa \hat{\omega}^{(h)}$ between any phase and the phase labeled $h_{0}$ :

$$
\frac{i \kappa\left(\hat{\omega}^{(h)}-\hat{\omega}^{\left(h_{0}\right)}\right)}{\varrho_{0}}=-\chi \frac{\left(\hat{\tau}^{(h)}\right)^{2}-\left(\hat{\tau}^{\left(h_{0}\right)}\right)^{2}}{\frac{4 \varrho_{0}^{2} L}{\kappa}-i \varrho_{0}\left(\hat{\omega}^{(h)}+\hat{\omega}^{\left(h_{0}\right)}\right)} .
$$

In the incompressible limit, the density in each phase must be $\varrho_{0}$, accordingly $\hat{\omega}^{(h)}=i \varrho_{0}$ for each $h$, such that for $\kappa \rightarrow \infty$, one obtains

$$
\frac{i \kappa\left(\hat{\omega}^{(h)}-\hat{\omega}^{\left(h_{0}\right)}\right)}{\varrho_{0}}=-\chi \frac{\left(\hat{\tau}^{(h)}\right)^{2}-\left(\hat{\tau}^{\left(h_{0}\right)}\right)^{2}}{2 \varrho_{0}^{2}},
$$

i.e., a quadratic dependence of the generalized density differences on the $A$ excess amplitudes, similar to eq. (3.24). Then, by multiplying numerator and denominator of eqs. 3.63a with a factor $\exp \left(-i L \kappa \hat{\omega}^{\left(h_{0}\right)} /\left(2 \varrho_{0}\right)\right)$ and inserting eq. 3.66), we arrive at the set of implicit equations for the amplitudes in the incompressible limit,

$$
\frac{\hat{\tau}^{(h)}}{\varrho_{0}}=\sum_{c} \tilde{q}_{c} \frac{\tilde{p}_{c}}{z_{c}} \exp \left\{\tilde{q}_{c} \frac{L \chi}{2 \varrho_{0}} \hat{\tau}^{(h)}-\frac{L \chi}{4 \varrho_{0}^{2}}\left(\hat{\tau}^{(h)}\right)^{2}\right\},
$$

with the component partition functions

$$
z_{c}=\sum_{h^{\prime}} v^{\left(h^{\prime}\right)} \exp \left\{\tilde{q}_{c} \frac{L \chi}{2 \varrho_{0}} \hat{\tau}^{\left(h^{\prime}\right)}-\frac{L \chi}{4 \varrho_{0}^{2}}\left(\hat{\tau}^{\left(h^{\prime}\right)}\right)^{2}\right\} .
$$

The saddle-point values of the detailed component densities from eq. 3.57$),\left\langle\varrho_{c}^{(h)}\right\rangle_{\mathcal{H}}$, are contained in eq. (3.67) as

$$
\frac{\left\langle\varrho_{c}^{(h)}\right\rangle_{\mathcal{H}}}{\varrho_{0}}=\frac{\tilde{p}_{c}}{z_{c}} \exp \left\{\tilde{q}_{c} \frac{L \chi}{2 \varrho_{0}} \hat{\tau}^{(h)}-\frac{L \chi}{4 \varrho_{0}^{2}}\left(\hat{\tau}^{(h)}\right)^{2}\right\} .
$$

The condition that the sum $\sum_{c}()$ over all component densities in one phase must be $\varrho_{0}$ in the incompressible case, is important in an iterative solution algorithm used to determine the volume fractions.

\subsubsection{Two symmetric homogeneous phases}

Now, we address the case relevant for our phase coexistence scheme later, viz., two, $A$ and $B$-rich, homogeneous phases in $A \leftrightharpoons B$ exchange-symmetric distributions of block copolymers. The ansatz consistent with the symmetry is for two homogeneous phases in equally sized regions of the system, with $A$ excess values of equal magnitude but opposite sign, $\hat{\tau}^{(1,2)}= \pm \hat{\tau}_{\mathrm{h}}$. With this ansatz, the component partition functions from eq. (3.68) are

$$
z_{c}=\exp \left\{-\frac{L \chi}{4 \varrho_{0}^{2}} \hat{\tau}_{\mathrm{h}}^{2}\right\} \cosh \left\{\tilde{q}_{c} \frac{L \chi}{2 \varrho_{0}} \hat{\mathrm{\tau}}_{\mathrm{h}}\right\},
$$

and the implicit equation for the $A$ excess (order-parameter) amplitude is

$$
\frac{\hat{\tau}_{\mathrm{h}}}{\varrho_{0}}=2 \sum_{c=0}^{\left\lceil\frac{Q}{2}\right\rceil} \tilde{q}_{c} \tilde{p}_{c} \tanh \left\{\tilde{q}_{c} \frac{L \chi \hat{\tau}_{\mathrm{h}}}{2 \varrho_{0}}\right\}
$$


(since components $c$ and $Q-c$ have equal probabilities in the symmetric case). The free-energy density becomes

$$
f_{\mathrm{h}}=\frac{L \chi}{4 \varrho_{0}^{2}} \hat{\tau}_{\mathrm{h}}^{2}-2 \sum_{c=0}^{\left\lceil\frac{Q}{2}\right\rceil} \tilde{p}_{c} \ln \cosh \left\{\tilde{q}_{c} \frac{L \chi \hat{\tau}_{\mathrm{h}}}{2 \varrho_{0}}\right\}+\sum_{\nu} p_{\nu} \ln p_{\nu}
$$

\subsubsection{Two symmetric homogeneous phases in random triblock copolymers}

For random triblock copolymers, $Q=3$, four different compositions are possible and define the components according to eq. (3.56). Relating the component probabilities $\tilde{p}_{c}$ in a symmetric distribution to the probabilities of the sequence classes defined in eq. (3.42) (needed for the description of a lamellar phase),

$$
\tilde{p}_{0}=\tilde{p}_{3}=\frac{p_{1}}{2}, \quad \tilde{p}_{1}=\tilde{p}_{2}=\frac{p_{2}+p_{3}}{2}=\frac{1-p_{1}}{2} .
$$

the free-energy density of two homogeneous phases takes the form

$$
\begin{aligned}
f_{\mathrm{h}}= & \frac{L \chi \hat{\tau}_{\mathrm{h}}^{2}}{4 \varrho_{0}^{2}}-\left(1-p_{1}\right) \ln \cosh \left\{\frac{L \chi \hat{\tau}_{\mathrm{h}}}{6 \varrho_{0}}\right\}-p_{1} \ln \cosh \left\{\frac{L \chi \hat{\tau}_{\mathrm{h}}}{2 \varrho_{0}}\right\} \\
& +\sum_{\nu} p_{\nu} \ln p_{\nu}, \text { provided } L \chi>L \chi_{0}:=\frac{2}{m_{2}}=\frac{18}{1+8 p_{1}}
\end{aligned}
$$

(with the moments $m_{2,1}=1$ and $m_{2,2}=m_{2,3}=1 / 9$ ). For macroscopic phase separation, the homopolymer concentration $p_{1}$ is seen to be the only relevant parameter of the triblock sequence distribution. In eq. (3.74), the amplitude $\hat{\tau}_{\mathrm{h}}$ is determined by the implicit equation

$$
\frac{\hat{\tau}_{\mathrm{h}}}{\varrho_{0}}=\frac{1-p_{1}}{3} \tanh \left\{\frac{L \chi \hat{\tau}_{\mathrm{h}}}{6 \varrho_{0}}\right\}+p_{1}\left\{\tanh \frac{L \chi \hat{\tau}_{\mathrm{h}}}{2 \varrho_{0}}\right\} .
$$

Along with gradual exchange of $B$ - with $A$-rich subspecies within one sequence class in the $A$-rich phase, conversely for the $B$-rich phase, the amplitude of macroscopic phase separation increases. 


\section{Three-phase coexistence with sequence fractionation}

Here, we analyze the possible coexistence of phases with different morphologies via sequence selection among coexisting subsystems. This mechanism is called sequence fractionation. Our aim is to calculate the free energy of the coexistence and compare it to that of the global, ordered state which forms upon increasing incompatibility in the disordered state of the overall sequence distribution. Optimization of our multiphase free energy will show that both global macroscopic and global lamellar phase separation, upon further increasing incompatibility, become unstable toward a coexistence of two homogeneous, A- and B-rich phases and one lamellar phase. Due to sequence fractionation, the coexistence of subsystems with different morphologies can attain a lower free energy than one global ordered state. Starting from global macroscopical phase separation, the two homogeneous cloud phases (we adopt the terms 'cloud' and 'shadow' phase from [110]) expel mainly alternating block copolymer sequences with internal $A-B$ contacts into an emerging third, lamellar shadow phase. In the subsystem, the accumulation of alternating sequences induces a peak of the structure function at nonzero wave number and thus a lamellar structure, whereas the structure function of the global distribution indicates macroscopic phase separation. Conversely, a lamellar (cloud) phase expels chiefly homopolymers into two additional homogeneous shadow phases. The deviation of the shadow's sequence distribution from the global, $\lambda$-defined distribution is found to be discontinuous (cf. sec. 5.3 below). The topology of the phase diagrams we will derive is shown in fig. 4.1 .

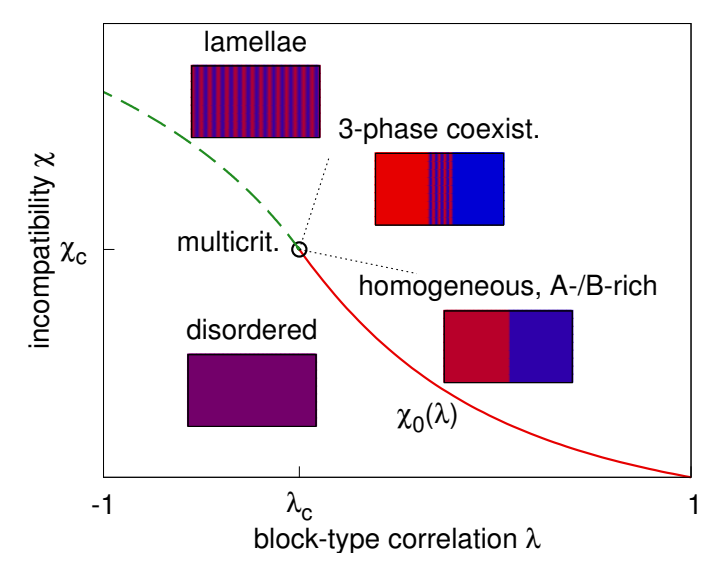

Figure 4.1: Qualitative phase diagram of symmetric random block copolymers with fractionated threephase coexistence. Global instabilities of the disordered state: solid (red) line: macroscopic phase separation for $\lambda>\lambda_{c}$, dashed (green) line: lamellar order-disorder transition (ODT) for $\lambda<\lambda_{c}$. Sequence fractionation creates an in-between state with three coexisting phases. 


\subsection{General fractionation ansatz}

Starting from a system with given global sequence probabilities $\left\{p_{\nu}\right\}$ in a volume $V$, we consider a partitioning into subsystems, indexed by $P$, with different morphologies, whose equilibrium sequence probabilities may deviate from the global ones (fractionation). The variable sequence probabilities $n_{\nu}^{(P)}:=N_{\nu}^{(P)} / N^{(P)}$, also called concentrations, and volume fractions $v^{(P)}:=V^{(P)} / V$ of all coexisting subsystems have to be obtained by minimization of the total free energy. With incompressibility, the volume fractions correspond to number fractions, $v^{(P)}=N^{(P)} / N$. As in chapter 3, we will consider the free energy per chain, i.e., the free-energy density. Assuming, as usual, the thermodynamic limit for each subsystem, the total free-energy density $f_{\text {frac }}$ is a weighted sum of the free-energy densities of the subsystems,

$$
f_{\text {frac }}\left(\left\{v^{(P)}, n_{\nu}^{(P)}\right\}\right)=\sum_{P} v^{(P)} f^{(P)}\left(\left\{n_{\nu}^{(P)}\right\}\right) \stackrel{!}{=} \min .
$$

Since we accounted for the indistinguishability of copolymers of equal sequence already in the calculation of the free-energy density of one ordered state, cf. eq. (3.2), there is no need to introduce additional mixing entropy terms for the case of coexisting subsystems.

\subsection{Symmetry considerations}

The $A \leftrightharpoons B$ exchange symmetry, valid for the globally symmetric distributions, must hold for each of the coexisting subsystems, too. In this context, we consider as only one subsystem the set of homogeneous phases or domains in macroscopic phase separation and refer to it as a homogeneous bi-phase in this chapter. Hence, in globally symmetric

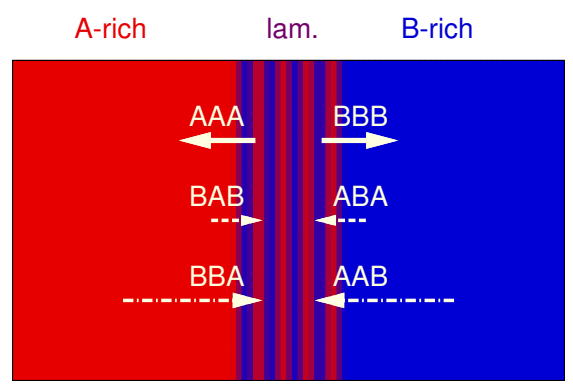

Figure 4.2: Sequence class exchange between homogeneous phases (macroscopic phase separation) and lamellae (microphase separation) in random triblock copolymers; arrows indicate expected net fluxes and show the symmetry with respect to $A$ - and $B$-rich representative.

distributions, the sequence exchange between subsystems of different morphologies can take place only within the sequence classes (introduced in appendix B.1), the $A$ - and $B$-rich representatives always being transferred in equal amounts. Figure 4.2 illustrates the situation we expect for symmetric triblock copolymers, which fall into the three sequence classes defined in eq. (3.42). Especially, the two homogeneous phases' difference in $A$ content needs not to be taken into account in the sense of sequence class 
fractionation. The sums over individual sequences in the lamellar free-energy density, e.g., in eq. (3.50), can be obviously reduced to sums over sequence classes, since one sequence and its complement have equal concentrations and identical structure functions, cf. sec. 3.3.3. This symmetry reduces the independent probabilities in analyzing the coexistence conditions, and in the following, we will use the index $\nu$ for sequence classes.

\subsection{Constraints at fixed global sequence distribution}

The constraints that impose incompressibility, number conservation, and the fixed set of global concentrations $\left\{p_{\nu}\right\}$ are

$$
\begin{aligned}
1 & =\sum_{P} v^{(P)}, \\
1 & =\sum_{\nu} n_{\nu}^{(P)}, \\
p_{\nu} & =\sum_{P} v^{(P)} n_{\nu}^{(P)} .
\end{aligned}
$$

For Markovian sequence distributions, the global concentrations at a given $\lambda$ are $\left\{p_{\nu}(\lambda)\right\}$. The constraints eq. (4.2) reduce further the number of independent parameters of the coexisting subsystems.

\subsection{Three-phase coexistence conditions}

Now, we derive the equilibrium conditions specifically for two coexisting subsystems in a symmetric distribution: one bi-phase, comprising two homogeneous, $A$ - and $B$-rich phases, and one with lamellar structure. This state is called three-phase coexistence.

In general, the free-energy minimization can be performed with Lagrange multipliers for each of the constraints, or, the number of variables can be reduced immediately by inserting the constraints eqs. (4.2), owing to their simple form. Here, we take a route in-between. First, we use explicitly constraint eq. 4.2a to eliminate one of the volume fractions, and eqs. (4.2b) to eliminate one concentration per subsystem.

Following eq. (4.1), the free-energy density $f_{\text {frac }}$ is then a linear combination of the free-energy densities $f_{\mathrm{m}}$, eq. (3.54) (or eq. $(3.50)$ ), of a lamellar phase with volume fraction $v$, and $f_{\mathrm{h}}$, eq. (3.72), of a homogeneous bi-phase with volume fraction $(1-v)$ :

$$
f_{\text {frac }}:=v f_{\mathrm{m}}\left(\left\{n_{\nu}^{(\mathrm{m})}\right\}^{\prime}\right)+(1-v) f_{\mathrm{h}}\left(\left\{n_{\nu}^{(\mathrm{h})}\right\}^{\prime}\right),
$$

where $\left\{n_{\nu}^{(\mathrm{m})}\right\}^{\prime}$ denotes the set of independent concentrations. Similarly, $\sum_{\nu}^{\prime}$ will be used to denote the sum over all sequence classes $\nu$ whose concentrations have been chosen as independent variables.

Second, via introducing Lagrange multipliers $\mu_{\nu}$ for the constraints eq. (4.2c), the function $\tilde{f}_{\text {frac }}$ to be extremized becomes

$$
\tilde{f}_{\text {frac }}=v f_{\mathrm{m}}\left(\left\{n_{\nu}^{(\mathrm{m})}\right\}^{\prime}\right)+(1-v) f_{\mathrm{h}}\left(\left\{n_{\nu}^{(\mathrm{h})}\right\}^{\prime}\right)-\sum_{\nu}^{\prime} \mu_{\nu}\left(v n_{\nu}^{(\mathrm{m})}+(1-v) n_{\nu}^{(\mathrm{h})}\right) .
$$


Minimization of $\tilde{f}_{\text {frac }}$ requires the partial derivatives to vanish. Differentiation with respect to the independent concentrations gives

$$
\mu_{\nu}=\frac{\partial f_{\mathrm{m}}}{\partial n_{\nu}^{(\mathrm{m})}}, \quad \mu_{\nu}=\frac{\partial f_{\mathrm{h}}}{\partial n_{\nu}^{(\mathrm{h})}},
$$

and differentiation with respect to the volume fraction gives

$$
f_{\mathrm{m}}-\sum_{\nu}^{\prime} n_{\nu}^{(\mathrm{m})} \mu_{\nu}=f_{\mathrm{h}}-\sum_{\nu}^{\prime} n_{\nu}^{(\mathrm{h})} \mu_{\nu}
$$

with

$$
n_{\nu}^{(\mathrm{h})}=\frac{p_{\nu}-v n_{\nu}^{(\mathrm{m})}}{1-v}
$$

according to eqs. 4.2c). Equations 4.5a demand, for each sequence class $\nu$, the chemical potentials in the coexisting subsystems to be equal, and eq. $4.5 \mathrm{~b}$ states that the grand free-energy density be equal in both subsystems. Since the concentrations $n_{\nu}^{(\mathrm{h})}$ are thus eliminated, we can simplify the notation and write $n_{\nu}:=n_{\nu}^{(\mathrm{m})}$ for the concentrations in the lamellar phase.

Given the block-type correlation $\lambda$ of the global distribution and the incompatibility $L \chi$, the next step is to insert into the equation system (4.5) the derivatives $\mu_{\nu}$ of the subsystem's free-energy densities $f_{\mathrm{m}}$ and $f_{\mathrm{h}}$. On the basis of eqs. (3.50), (3.54), and (3.72), we have calculated these derivatives analytically (setting $p_{\nu} \rightarrow n_{\nu}^{(\mathrm{m})}$, respectively $p_{\nu} \rightarrow n_{\nu}^{(\mathrm{h})}$ ), see sec. 4.4 .2 specializing to the triblock system below. At this point, the computation of structure functions and moments for individual sequence classes, exemplified in sec. 3.3.3, turns out to be vital. Especially, eq. (3.54) requires the sequence-specific fourth-order structure functions, cf. appendix C.6. Next, eqs. 4.5 have to be solved for the independent parameters, $\left(v,\left\{n_{\nu}\right\}^{\prime}\right)$, that determine the two subsystems, lamellae and homogeneous bi-phase.

Obviously, the subsystems can coexist with nonzero volume fractions only if $f_{\text {frac }}$, eq. (4.3), reaches a lower value than the minimum of $f_{\mathrm{m}}\left(\left\{p_{\nu}(\lambda)\right\}, \chi\right)$ and $f_{\mathrm{h}}\left(\left\{p_{\nu}(\lambda)\right\}, \chi\right)$, i.e., than the free-energy density of a global ordered state with $\lambda$-distribution:

$$
\left.\Delta f_{\text {frac }}:=f_{\text {frac }}\left(v,\left\{n_{\nu}\right\}^{\prime} \mid \lambda, \chi\right)\right)-\min \left[f_{\mathrm{m}}\left(\left\{p_{\nu}(\lambda)\right\}, \chi\right), f_{\mathrm{h}}\left(\left\{p_{\nu}(\lambda)\right\}, \chi\right)\right] \stackrel{!}{<} 0 .
$$

Hence, this inequality defines the coexistence region in the $\lambda-\chi$ plane, and the set of points at which $\Delta f_{\text {frac }}$ vanishes constitutes the boundary of this region, indicating an instability of the system. The numerical procedure to locate, within the $\lambda-\chi$ plane, points of solutions conforming the condition (4.6) is described in sec. 4.4 .3 below. Anticipating our results shown in chapter 5 , we indeed find an extended region of threephase coexistence, see, e.g., the phase diagram for random continuous-chain triblock copolymers in fig. 5.9. In the $\lambda-\chi$ plane, the three-phase region is located above the critical line $L \chi_{0}(\lambda)$ of the disordered state, cf. fig. 4.1, separating the region of global lamellar states which extends to $\lambda \rightarrow-1$ and the region of homogeneous bi-phase states which extends to $\lambda \rightarrow+1$. Both regions of global ordered states and the three-phase region meet at the multicritical point $\left(\lambda_{c}, L \chi_{c}\right)$. 


\subsubsection{Transition lines to the three-phase region}

Upon gradually increasing $\chi$ in the global homogeneous bi-phase, the latter becomes unstable at a critical incompatibility $\chi^{(\mathrm{h})}$, and in the $\lambda$ - $\chi$ plane, the function $\chi^{(\mathrm{h})}(\lambda)$ represents the transition line to the three-phase region determined by 4.6 . Conversely, starting in the three-phase region, the transition line is approached by decreasing $\chi$ and is defined by the limit

$$
\Delta f_{\text {frac }}=f_{\text {frac }}\left(v,\left\{n_{\nu}\right\}^{\prime} \mid \lambda, \chi\right)-f_{\mathrm{h}}\left(\left\{p_{\nu}(\lambda)\right\}, \chi\right) \underset{\chi \rightarrow \chi^{(\mathrm{h})}}{\longrightarrow} 0 .
$$

This means that the lamellar phase must disappear (or become indistinguishable from the homogeneous bi-phase) on the line $\chi^{(\mathrm{h})}(\lambda)$. In principle, this can be realized in three ways, with the following implications for the lamellar phase:

$$
\begin{aligned}
\text { volume fraction vanishes: } & v \rightarrow 0, \\
\text { concentrations approach } \lambda \text {-defined ones: } & n_{\nu} \rightarrow p_{\nu}(\lambda) \text {, or } \\
\text { amplitude vanishes: } & \hat{\tau}_{\mathrm{m}} \rightarrow 0
\end{aligned}
$$

Analysis of the free-energy densities eqs. (3.50) (or (3.54) and (3.72) contributing to $\Delta f_{\text {frac }}$ and solutions to eqs. (4.5) show that only the first limit is realized. This limit excludes the alternatives $4.8 \mathrm{~b})$ and $(4.8 \mathrm{c})$, i.e., $n_{\nu} \neq p_{\nu}(\lambda)$ and $\hat{\tau}_{\mathrm{m}} \neq 0$ on the transition line $\chi^{(\mathrm{h})}(\lambda)$. On this line, the lamellar phase with vanishing volume fraction is called a shadow phase, the coexisting homogeneous bi-phase a cloud phase. With the information of a lamellar shadow phase, the computation of the line $\chi^{(\mathrm{h})}(\lambda)$ is simplified, in that we can transform eqs. 4.5 into a set of equations for the parameters $\left\{n_{\nu}\right\}, \chi^{(\mathrm{h})}$ at fixed $\lambda$.

While the concentrations $\left\{n_{\nu}\right\}$ of the lamellar shadow deviate from the $\lambda$-defined ones on the line $\chi^{(\mathrm{h})}(\lambda)$, those in the homogeneous bi-phase cloud are restricted to the $\lambda$-defined concentrations. Approaching this line from the three-phase region, the deviations of the sequence concentrations in the majority subsystem from the $\lambda$-defined concentrations tend to zero linearly in $v$,

$$
n_{\nu}^{(\mathrm{h})}-p_{\nu}(\lambda)=\left(p_{\nu}(\lambda)-n_{\nu}\right) v+\mathcal{O}\left(v^{2}\right),
$$

according to eqs. 4.5c). For $v>0$, both subsystems acquire sequence concentrations deviating from the global ones.

The phase transition line $\chi^{(\mathrm{m})}(\lambda)$ from the region of global lamellar states to the three-phase state is determined analogously to eq. (4.7) by the limit

$$
\Delta f_{\text {frac }}=f_{\text {frac }}\left(v,\left\{n_{\nu}\right\}^{\prime} \mid \lambda, \chi\right)-f_{\mathrm{m}}\left(\left\{p_{\nu}(\lambda)\right\}, \chi\right) \underset{\chi \rightarrow \chi^{(\mathrm{m})}}{\longrightarrow} 0
$$

where now $v$ and $\left\{n_{\nu}\right\}^{\prime}$ are volume fraction, respectively independent concentrations in the (disappearing) homogeneous bi-phase. Also in this case, $v \rightarrow 0, n_{\nu} \neq p_{\nu}(\lambda)$, and $\hat{\tau}_{\mathrm{h}} \neq 0$ on the line $\chi^{(\mathrm{m})}(\lambda)$, on which accordingly, the homogeneous bi-phase is called a shadow phase. 
In constructing the free-energy superposition eq. (4.3), we assume for the free-energy density of the lamellar subsystem the one-mode ansatz and the expansion in the orderparameter amplitude leading to eq. (3.50). In contrast, the free-energy density of the homogeneous bi-phase, eq. (3.72), is exact within the "crushed polymer" picture and hence is in its validity not limited to small amplitudes. As a consequence, the result for the transition line $\chi^{(\mathrm{h})}(\lambda)$ of the homogeneous bi-phase cloud might be more reliable than that for the line $\chi^{(\mathrm{m})}(\lambda)$.

\subsubsection{Random triblock copolymers}

For random triblock copolymers, we solve the equation system 4.5 with the lamellar free-energy density $f_{\mathrm{m}}$ both in the simplified form, eq. 3.50), and in the form obtained by the enhanced theory, eq. (3.54). For the free-energy density $f_{\mathrm{h}}$ of the homogeneous phases, we use the expression eq. (3.74). Here, the solution strategy is sketched for the vicinity of the transition from the global homogeneous bi-phase state to the threephase region. In this case, the independent variables are chosen as the parameters of the lamellar subsystem, $v, n_{2}:=n_{2}^{(\mathrm{m})}$ and $n_{3}:=n_{3}^{(\mathrm{m})}$, cf. the sequence classes in sec. 3.3.3. At a given $(\lambda, \chi)$, we determine the (candidate) parameter vector $\left[v, n_{2}, n_{3}\right]$ as the solution to the equation system eqs. 4.5), i.e., as a zero of the gradient vector $\nabla_{\left(v, n_{2}, n_{3}\right)} f_{\text {frac }}$. Subsequently, the solution vector has to be tested to be within the domain of definition, cf. appendix D.2, and $f_{\text {frac }}$ has to be verified to be smaller than $f_{\mathrm{h}}$, cf. inequality (4.6).

In an attempt to visualize the differentiations which have to be computed analytically prior to solve eqs. (4.5), we write out explicitly the expression $f_{\text {frac }}$, cf. eq. (4.3), for the triblock system, using the simplified version eq. (3.50) for $f_{\mathrm{m}}$ :

$$
\begin{aligned}
& f_{\text {frac }} \\
= & v\left\{\sum_{\nu=1}^{3} n_{\nu} \ln n_{\nu}-\frac{\left(\sum_{\nu=1}^{3} n_{\nu} S_{\nu}\left(k_{\mathrm{m}}^{2}\right)-2 L / \chi\right)^{2} / L^{4}}{\left(\sum_{\nu=1}^{3} n_{\nu} m_{2, \nu}\right)^{2}+\sum_{\nu=1}^{3} n_{\nu} m_{4, \nu}}\right\}+(1-v) \sum_{\nu=1}^{3} n_{\nu}^{(\mathrm{h})} \ln n_{\nu}^{(\mathrm{h})} \\
& +(1-v)\left\{\frac{L \chi \hat{\tau}_{\mathrm{h}}^{2}}{4 \varrho_{0}^{2}}-\left(n_{2}^{(\mathrm{h})}+n_{3}^{(\mathrm{h})}\right) \ln \cosh \left\{\frac{L \chi \hat{\tau}_{\mathrm{h}}}{6 \varrho_{0}}\right\}-n_{1}^{(\mathrm{h})} \ln \cosh \left\{\frac{L \chi \hat{\tau}_{\mathrm{h}}}{2 \varrho_{0}}\right\}\right\} .
\end{aligned}
$$

The structure functions $S_{\nu}\left(k^{2}\right)$ are given in sec. 3.3.3, and the moments are $m_{2,2}=$ $m_{2,3}=1 / 9, m_{2,1}=1$ and $m_{4,2}=m_{4,3}=1 / 81, m_{4,1}=1$. In eq. (4.11), the lamellar wave number $k_{\mathrm{m}}$ is a function of $n_{2}$ and $n_{3}$ via

$$
k_{\mathrm{m}}:=\underset{k>0}{\operatorname{argmax}} \sum_{\nu} n_{\nu} S_{\nu}\left(k^{2}\right),
$$

but needs not to be differentiated with respect to $n_{2}, n_{3}$ in the system (4.5), since per definition $0=\left.\left(\partial \sum_{\nu} n_{\nu} S_{\nu}\left(k^{2}\right) / \partial k\right)\right|_{k=k_{\mathrm{m}}}$. Similarly, the amplitude of the homogeneous phases, $\hat{\tau}_{\mathrm{h}}$, determined by the implicit equation

$$
\frac{\hat{\tau}_{\mathrm{h}}}{\varrho_{0}}=\frac{n_{2}^{(\mathrm{h})}+n_{3}^{(\mathrm{h})}}{3} \tanh \left\{\frac{L \chi \hat{\tau}_{\mathrm{h}}}{6 \varrho_{0}}\right\}+\left(1-n_{2}^{(\mathrm{h})}-n_{3}^{(\mathrm{h})}\right)\left\{\tanh \frac{L \chi \hat{\tau}_{\mathrm{h}}}{2 \varrho_{0}}\right\},
$$


depends on the concentrations $n_{2}^{(\mathrm{h})}, n_{3}^{(\mathrm{h})}$, but needs not to be differentiated with respect

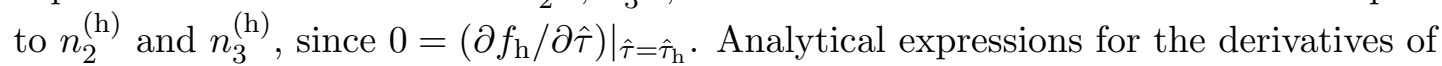
$f_{\mathrm{m}}$ and $f_{\mathrm{h}}$ for the triblock system are given in appendix D.1.

The fact that the second line of eq. 4.11) depends solely on the sum of the concentrations $n_{2}^{(\mathrm{h})}$ and $n_{3}^{(\mathrm{h})}$ simplifies the system 4.5 for the computation of the parameters $n_{2}, n_{3}$, and $\chi^{(\mathrm{h})}$ on the three-phase boundary, cf. sec. 4.4.1, in random triblocks.

For the case that the homogeneous bi-phase is expected to be the minority subsystem (or the shadow phase), the parameters $v^{(\mathrm{h})}, n_{2}^{(\mathrm{h})}$, and $n_{3}^{(\mathrm{h})}$ are chosen as independent variables, and the above-sketched calculations are performed analogously.

\subsubsection{Solution algorithm}

In order to solve the system (4.5), we employ a Newton-type procedure using the following steps, exemplified for random triblock copolymers and for the variables $\left(v, n_{2}, n_{3}\right)$ of the conjectured lamellar minority subsystem:

1. At a given set $(\lambda, \chi)$ of block-type correlation and incompatibility, guess start parameter vector $\boldsymbol{x}_{0}:=\left[v_{0}, n_{2,0}, n_{3,0}\right]^{T}(\lambda, \chi)$. The sensitivity regarding the start vector impedes completely automatized scans in the $\lambda-\chi$ plane.

2. Iteratively, apply Newton scheme

$$
\boldsymbol{x}_{1}=\boldsymbol{x}_{0}-\mathrm{H}^{-1}\left(\boldsymbol{x}_{0}\right) \nabla f_{\text {frac }}\left(\boldsymbol{x}_{0}\right),
$$

with $\mathrm{H}$ the Hessian of the system 4.5 .

3. Stop if either the desired relative precision $\epsilon:=\frac{\left|\boldsymbol{x}_{1}-\boldsymbol{x}_{0}\right|}{\left|\boldsymbol{x}_{0}\right|}$ or a given maximal number of iterations has been reached. In the latter case, and if $\mathrm{H}$ gets singular during the iteration, restart from step 1 .

4. To ensure that $f_{\text {frac }}\left(\boldsymbol{x}_{1}\right)$ is a minimum, check $\mathrm{H}$ for positive definiteness, i.e. calculate its eigenvalues.

5. Once obtained a solution vector $\left[v, n_{2}, n_{3}\right]$, check it to be included in the set (D.5). From the concentrations $n_{2}, n_{3}$, calculate $k_{\mathrm{m}}\left(n_{2}, n_{3}\right)$, respectively $k_{\text {opt }}\left(n_{2}, n_{3} \mid \chi\right)$. Particularly, the critical incompatibility $L \chi_{0}\left(n_{2}, n_{3}\right)=2 L^{2} /\left(\sum_{\nu} n_{\nu} S_{\nu}\left(k_{\mathrm{m}}^{2}\right)\right)$ of the assumed lamellar subsystem must be smaller than $L \chi$.

Convergence, especially while approaching the multicritical point $\left(\lambda_{c}, L \chi_{c}\right)$, can be achieved only for start vectors very close to the actual solution. Therefore, proceeding on a three-phase transition line (see section 4.4.1) toward $\left(\lambda_{c}, L \chi_{c}\right)$, we use the solution at one value of $\lambda$ as the start vector for the solution at an adjacent value of $\lambda$. The resolution for $\lambda$ is chosen between $5 \cdot 10^{-4}$ far from $\lambda_{c}$ and $10^{-5}$ near $\lambda_{c}$, and between $10^{-3}$ and $10^{-4}$ for $L \chi$. In the vicinity of $\left(\lambda_{c}, L \chi_{c}\right)$, entries of the start vector have to be even closer to the actual solution and are obtained by extrapolating solutions on the boundary line. Finally, the result vector is calculated with a relative precision $\epsilon=10^{-12}$ of its modulus. Uniqueness of solutions of the nonlinear equation system (4.5) cannot be proven here. However, the determined $\chi^{(\mathrm{h})}(\lambda)$, respectively $\chi^{(\mathrm{m})}(\lambda)$, are lower bounds 
for the transition incompatibilities to three-phase coexistence in our model, since at each $\lambda$, we initialize the scan of the domain of definition, eq. (D.5), with the global concentrations $p_{\nu}(\lambda)$ at $\chi_{0}(\lambda)$. 


\section{Phase behavior with fractionation}

In this chapter, we present a selection of the results drawn from our fractionation theory, i.e., the three-phase transition lines and detailed information about the subsystems within the coexistence region of random symmetric triblock copolymers. While the focus here is on the coexistence of one subsystem with two homogeneous phases and one with lamellar structure, the phase diagrams naturally include the critical lines of global macroscopic and lamellar phase separations of the disordered state. The behavior at the multicritical point $\left(\lambda_{c}, L \chi_{c}\right)$, at which regions of the global ordered states, of the three-phase coexistence, and of the disordered state meet, is found to depend on the parameter $M$, the number of segments per block. Sequence distribution diagrams visualize the sequence fractionation among the coexisting subsystems and the deviations from the global Markovian sequence distribution. Also for random diblocks, a symmetric mixture of $A A$ and $B B$ with $A B$, we find a three-phase coexistence with subsystems of different $A B$ concentrations. A brief comparison with numerical SCFT results for continuous-chain triblocks is provided at the end of this chapter.

\subsection{Triblocks with a small number of segments per block}

Here, we discuss our results for the phase diagram with three-phase coexistence region for random symmetric triblock copolymers with $M=3$ segments per block, shown in fig. 5.1. This phase diagram is representative of the phase behavior of triblocks with $M<7$ segments per block. In order to explore the emergence and growth of the various phases, we follow the path indicated by arrows in the plot, starting at a supercritical block-type correlation $\lambda>\lambda_{c}=-1 / 3$. In this case, the instability of the disordered state is toward global macroscopic phase separation, as is indicated by the peak of the global structure function $S\left(k^{2}\right)$ at zero wave number (see the solid curve in the bottom inset of fig. 5.1), cf. the critical wave number eq. (3.33) and eq. (3.41). Upon increasing the incompatibility $L \chi$ along the bottom vertical arrow, the line $\chi^{(\mathrm{h})}$ (dotted) marks the onset of three-phase coexistence via a fractionated lamellar shadow phase (with volume fraction $v^{(\mathrm{m})}=0$ ) according to sec. 4.4.1. This lamellar shadow emerges with nonzero amplitude, and with nonzero wave number, since the sequence distribution in the lamellar subsystem is enriched in alternating sequences (see fig. 5.14 below) and hence $S\left(k^{2}\right)$ in the subsystem has a peak at nonzero $k$ (see also the individual $S_{\nu}\left(k^{2}\right)$ in fig. 3.4 . Upon further increase of the incompatibility (along the top vertical arrow), the lamellar volume fraction grows. Now, keeping $L \chi$ constant, and proceeding toward smaller values of $\lambda$ along the horizontal arrow, the volume fraction of the lamellar subsystem increases further. At some $\lambda<\lambda_{c}$, one reaches the boundary $\chi^{(\mathrm{m})}$ of three-phase coexistence, and lamellae take over to be the cloud phase with $v^{(\mathrm{m})}=1$. Consistently, starting at $\lambda<\lambda_{c}$ from the disordered state, the latter undergoes lamellar phase separation on the dashed part of the critical line $\chi_{0}(\lambda)$, due to the peak of $S\left(k^{2}\right)$ at a nonzero wave number in 


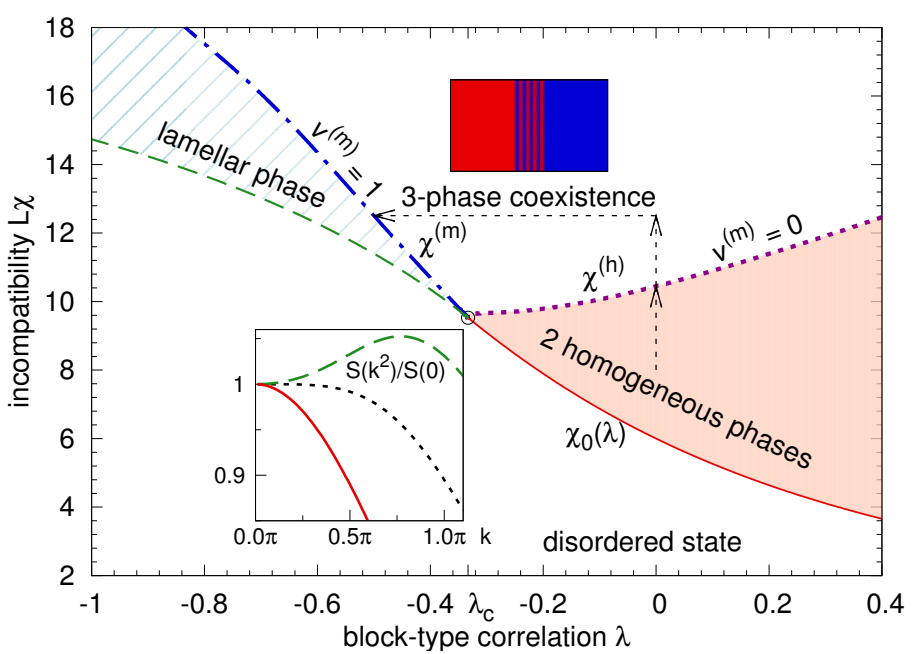

Figure 5.1: Phase diagram for random triblock copolymers with $M=3$ segments per block. The line $\chi_{0}(\lambda)$ indicates the instabilities of the disordered state: solid (red) macroscopic phase separation into two homogeneous, $A$ - and $B$-rich phases, dashed (green) lamellar phase separation or order-disorder-transition (ODT). The dotted (purple) line $\chi^{(\mathrm{h})}$ marks the transition to threephase coexistence, at which the two homogeneous phases are the cloud and the lamellar phase the shadow, the dot-dashed (blue) line $\chi^{(\mathrm{m})}$ the lamellar cloud boundary. A circle (o) marks the multicritical point. Bottom inset: second-order structure function in the global $\lambda$-distribution, at $\lambda=0$ (solid), at the critical correlation $\lambda_{c}=-1 / 3$ (dotted), and at $\lambda=-0.5$ (dashed). In this and the following plots, the length scale is the average chain size, $R_{\text {chain }}:=R_{e} / \sqrt{d}$. Top inset: Sketch of three-phase coexistence.

the $\lambda$-defined distribution (cf. the dashed curve in the bottom inset). Upon crossing the boundary line $\chi^{(\mathrm{m})}$ from the region of global lamellae, two additional homogeneous phases with a sequence distribution enriched in homopolymers appear as shadow.

At the critical block-type correlation $\lambda_{c}$, the maximum at $k_{0}=0$ of the global $S\left(k^{2}\right)$ broadens (see the dotted graph in the bottom inset in fig. 5.1), announcing the continuous growth of the instability's wave number from zero upon decreasing $\lambda$. We observe this Lifshitz character of the transition from global macroscopic to global lamellar phase separation for all random triblock systems with $M<7$, cf. the case discussed before eq. (3.41), while the exact position of the Lifshitz point $\left(\lambda_{c}, L \chi_{c}\right)$ depends on $M$. The Lifshitz point $\left(\lambda_{c}, L \chi_{c}\right)$ of diverging lamellar wavelength also limits the three-phase coexistence region toward low incompatibilities.

The phase diagram in fig. 5.1 has been computed using the simplified description eq. (3.50) of the lamellar free energy. Within this description, the wave number $k_{\mathrm{m}}$ of global lamellae (hatched region in fig. 5.1) at fixed $\lambda$ remains constant upon increasing $L \chi$. Consequently, the lamellar cloud boundary of three-phase coexistence is confined to the half-plane $\lambda \leq \lambda_{c}$. Upon crossing the boundary to three-phase coexistence at a given $\lambda$, the lamellar wave number can be shifted only via fractionation, by altering the sequence distribution of the lamellar subsystem. The change of the wave number inside the three-phase region, not shown here, is as follows: upon increasing $L \chi$, the sequence fractionation proceeds and thereby the wave number in the lamellar subsystem increases, i.e., the lamellar wavelength decreases. 
The lamellar wave numbers on the boundaries of the fractionated three-phase coexistence as a function of $\lambda$ are displayed in fig. 5.2. The wave number of the fractionated

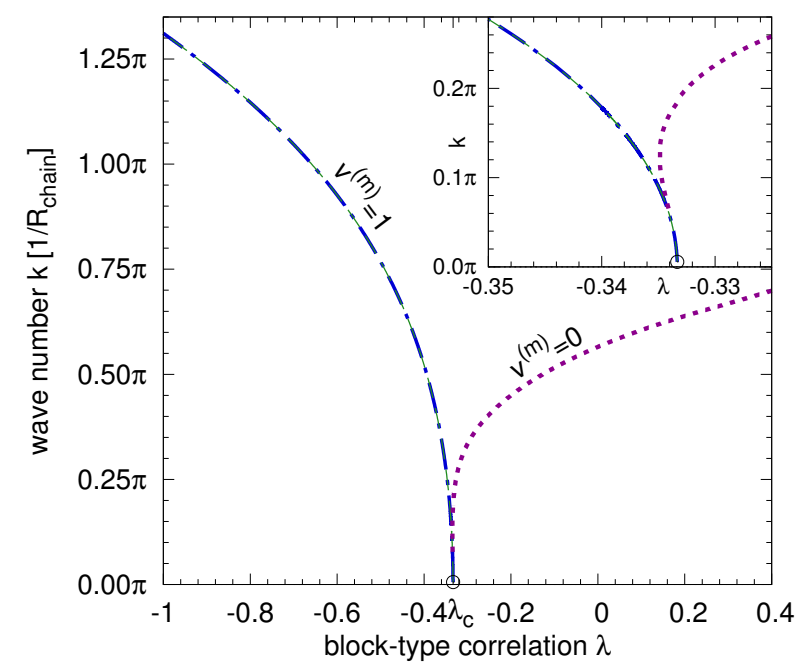

Figure 5.2: Wave number of the lamellar phase as a function of $\lambda$ for triblocks with $M=3$. Dot-dashed and dashed (blue, green) line: global lamellar phase and lamellar cloud at $\lambda<\lambda_{c}$, in the range $L \chi_{0} \leq L \chi \leq L \chi^{(\mathrm{m})}$ between the ODT and the transition line $v^{(\mathrm{m})}=1$ to three-phase coexistence (hatched region in fig. 5.1p. Dotted (purple) line: fractionated lamellar shadows $\left(v^{(\mathrm{m})}=0\right)$ on the line $\chi^{(\mathrm{h})}$, which is located mainly in the region $\lambda>\lambda_{c}$. Inset: blow-up around the multicritical point (o), showing the multi-valued curve for $\lambda \lesssim \lambda_{c}$.

lamellar subsystem vanishes at the Lifshitz point $\left(\lambda_{c}, \chi_{c}\right)$, as does the wave number of the global lamellar phase. The inset in fig. 5.2 shows in more detail the behavior of the lamellar shadow's wave number in the vicinity of the Lifshitz point. For $\lambda \lesssim \lambda_{c}$, the three-phase region can be entered, starting from a homogeneous bi-phase cloud, at two different values of $L \chi$, with two distinct wave numbers of the lamellar shadow.

This remarkable feature can be seen in the enlarged phase diagram fig. 5.3, showing the boundary lines and a map of the lamellar subsystem's volume fraction around the Lifshitz point. The lamellar shadow boundary of three-phase coexistence displays a

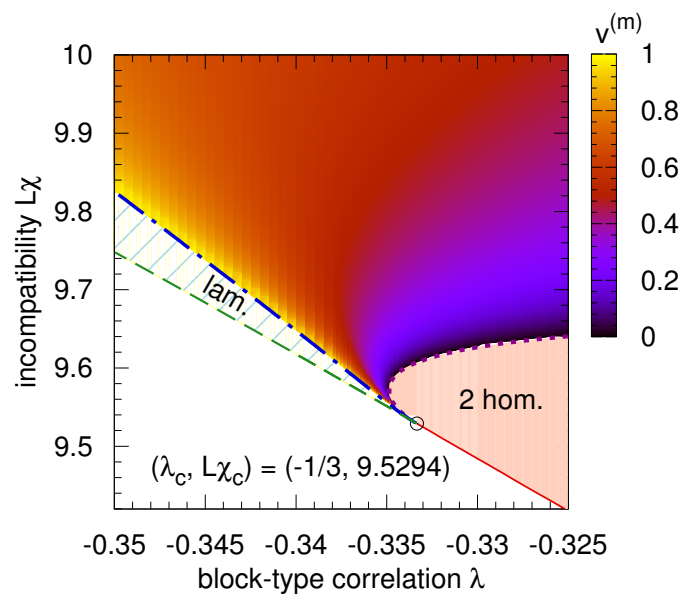

Figure 5.3: Three-phase coexistence boundaries around the multicritical (Lifshitz) point with volume fraction $v^{(\mathrm{m})}$ of the lamellar subsystem for triblocks with $M=3$. Line styles as in fig. 5.1

reentrant behavior: upon increasing $L \chi$ at fixed $\lambda \lesssim \lambda_{c}$, the line $\chi^{(\mathrm{h})}$ is crossed two times. (Reentrance has been observed in statistical physics, e.g., also for the melting of polydispersed hard spheres [10].) Especially, $\chi^{(\mathrm{h})}(\lambda)$ does not reach the multicritical point along a path $\lambda \downarrow \lambda_{c}$, but via a spiraling path invading the region $\lambda<\lambda_{c}$.

Another observation is that due to the presence of the three-phase coexistence region, 
the region of global lamellae with diverging wavelength in the vicinity of the Lifshitz point becomes effectively restricted to a very small region of the $\lambda-\chi$ plane. Given the alternative of sequence fractionation, a system with $\lambda \lesssim \lambda_{c}$, starting from the disordered state, reaches the homogeneous bi-phase nearly avoiding the global lamellar state with diverging wavelength, via intermediate three-phase states. At larger $L \chi$, the bi-phase is suppressed again in favor of three-phase coexistence, which allows for lamellae with finite wavelength in a fractionated subsystem.

\section{Four homogeneous phases in a triblock copolymer melt}

According to the results shown in fig. 5.1, the three-phase coexistence prevails in a large region of the $\lambda-\chi$ plane. Whether the three-phase coexistence persists at incompatibilities $L \chi$ far above the critical line $L \chi_{0}(\lambda)$ is a question beyond our scope here, since our expression for the lamellar free energy is reliable for small order-parameter amplitudes only. Within the multi-component or "crushed polymer" theory, which can account solely for macroscopic phase separation into multiple homogeneous phases, cf. sec. 3.5 we obtain for $\lambda>\lambda_{c}$ the scenario of a coexistence of four homogeneous phases at higher $L \chi$, e.g., for $\lambda=0$ at $L \chi=16.04$. More precisely, the theory yields a direct separation of two into four homogeneous phases, the two new phases emerging with zero volume fraction, but with finite amplitudes $\hat{\tau}_{h, 2}$ and $-\hat{\tau}_{h, 2}$ different from those of the already existing two phases, $\hat{\tau}_{h, 1}$ and $-\hat{\tau}_{h, 1}$. The finding that for triblocks, two homogeneous phases with increasing $L \chi$ are immediately followed by four within the multicomponent theory, is at first surprising, since earlier studies have suggested successive separations into two, three, ..., homogeneous phases for all symmetric copolymer melts [91, 125]. The absence of any sequence with equal $A$ and $B$ contents in the triblock system might explain why a third, homogeneous phase with zero amplitude does not appear. More than four homogeneous phases are impossible, since for triblocks there are only four components according to $A$ content. Taking into account structured phases, however, the fractionated three-phase coexistence discussed above sets in at lower $L \chi$ and thus precedes the coexistence of four homogeneous phases.

\subsubsection{Scaling of the order-parameter amplitude at the multicritical point}

With the numerical solution of the three-phase equilibrium conditions particularly for the transition lines, we are able to access the order-parameter amplitudes in the coexisting cloud and shadow phases. All these amplitudes vanish on approach to the multicritical point. The scaling of the order-parameter amplitude as a function of $\Delta \lambda:=\left|\lambda-\lambda_{c}\right|$ along the boundary lines of three-phase coexistence with $\lambda \uparrow \lambda_{c}$ is shown in fig. 5.4, for three cases: The amplitudes of fractionated lamellar shadows (coexisting with homogeneous clouds, on the part $\lambda<\lambda_{c}$ of the dotted line in fig. 5.3) are marked by open diamonds, those of lamellar cloud phases (on the the dot-dashed line in fig. 5.3 by solid triangles, those of the coexisting homogeneous shadows by open squares. According to the fit performed to the latter case, the amplitudes vanish approximately linearly in $\Delta \lambda$ at the Lifshitz point, indicating a critical exponent $\psi \approx 1$. The same exponent is obtained from the plots of the lamellar amplitudes. 


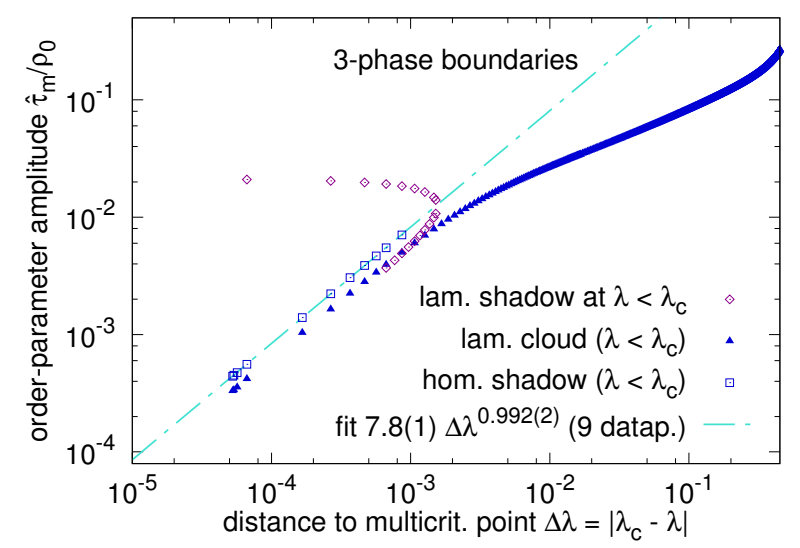

Figure 5.4: Decay of the orderparameter amplitudes as a function of $\left|\lambda-\lambda_{c}\right|$, proceeding toward the multicritical point on the boundaries of three-phase coexistence, which are located in the region $\lambda<\lambda_{c}$ for triblocks with $M=3$, see fig. 5.3 .

\section{Analytical determination of the critical exponent for $M<7$}

For triblocks with $M<7$, the Lifshitz behavior at the multicritical point $\left(\lambda_{c}, \chi_{c}\right)$ implies that the wave number $k_{\mathrm{m}}$ of the global lamellar instability and of the lamellar cloud vanishes continuously on approach to $\left(\lambda_{c}, L \chi_{c}\right)$, see also fig. 5.2. More specifically, $k_{\mathrm{m}}$ as a function of $\Delta \lambda$ is a square root,

$$
k_{\mathrm{m}} \propto(\Delta \lambda)^{1 / 2}, \lambda \uparrow \lambda_{c}
$$

as can be derived from an expansion in $\Delta \lambda$ of the maximum $S\left(k_{\mathrm{m}}^{2}\right)$ for a $\lambda$-distribution, cf. eq. (3.37). Given that the line $\chi_{0}(\lambda)$ has nonzero slope at $\left(\lambda_{c}, \chi_{c}\right)$, the decay of $k_{\mathrm{m}}$ with $\Delta \chi:=\chi-\chi_{c}$ occurs with exponent $1 / 2$, too.

The continuous decay of $k_{\mathrm{m}}$ at $\left(\lambda_{c}, \chi_{c}\right)$ allows us to analytically extract the exponent of the order-parameter amplitude in the lamellar subsystem at $\left(\lambda_{c}, \chi_{c}\right)$ from the equation system 4.5 for the lamellar cloud line $\chi^{(\mathrm{m})}$ (see sec. 4.4.1). In the vicinity of $\left(\lambda_{c}, \chi_{c}\right)$, we can solve this system for the deviations of the sequence concentrations in the homogeneous bi-phase shadow from the global concentrations, $\Delta n_{\nu}:=n_{\nu}^{(\mathrm{h})}-p_{\nu}(\lambda)$, with a power-series ansatz

$$
\Delta n_{\nu}(\Delta \lambda)=\sum_{j} c_{\nu j}(\Delta \lambda)^{j}
$$

In the representative case of $M=3$, consistent expansion up to $(\Delta \lambda)^{4}$ yields, along the line $\chi^{(\mathrm{m})}$ :

$$
\Delta n_{\nu}=-\frac{144 \sqrt{6}}{55}(\Delta \lambda)^{2}+\mathcal{O}\left((\Delta \lambda)^{3}\right), \quad \nu=2,3 .
$$

By inserting these concentration differences into an expansion of $\chi^{(\mathrm{m})}-2 L / S\left(k_{\mathrm{m}}^{2}\right)$, which determines the order-parameter amplitude $\hat{\tau}_{\mathrm{m}}$ (cf. eq. (3.48)) of the lamellar cloud phase with $\lambda$-distribution, we find the critical exponent $\psi=1$ for $\hat{\tau}_{\mathrm{m}}$,

$$
\hat{\tau}_{\mathrm{m}} \propto \Delta \lambda, \quad \lambda \uparrow \lambda_{c} .
$$

This result corroborates the findings for the exponent from the numerically determined values of the lamellar cloud amplitude. 
Moreover, we find that the slope of the instability line $\chi_{0}(\lambda)$ of the disordered state toward global lamellar phase separation and the slope of the lamellar cloud boundary $\chi^{(\mathrm{m})}(\lambda)$ of the three-phase coexistence region are equal at $\left(\lambda_{c}, \chi_{c}\right)$ :

$$
\chi^{(\mathrm{m})}(\lambda)-\chi_{0}(\lambda) \propto(\Delta \lambda)^{2}, \quad \lambda \uparrow \lambda_{c} .
$$

Hence, for $\lambda \lesssim \lambda_{c}$, there is a nearly direct transition from the disordered state to three-phase coexistence, as can be observed in fig. 5.3 .

\subsection{Triblocks with more segments per block and continuous-chain limit}

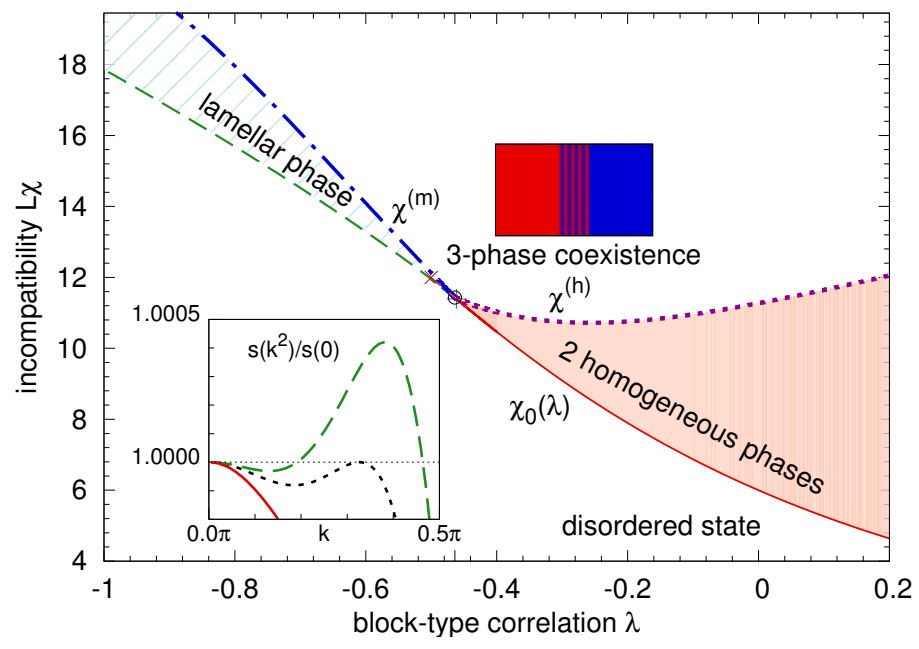

Figure 5.5: Phase diagram for continuous-chain triblocks, with line styles as in fig. 5.1 The multicritical point (o) is located at $\left(\lambda_{c}, L \chi_{c}\right)=$ $(-0.46400,11.316)$. Crosses indicate the end points of the lines of metastable, global phase separations of the disordered state, macroscopic for $\lambda<\lambda_{c}(\times)$ and lamellar for $\lambda>\lambda_{c}(+)$. Bottom inset: global second-order structure function $s\left(k^{2}\right)$ at $\lambda=-0.45$ (solid), at $\lambda_{c}$ (dotted), and at $\lambda=-0.47$ (dashed); the length scale is $R_{\text {chain }}$.

Representative of triblocks with $M \geq 7$ segments per block, we analyze the phase diagram for random, continuous-chain triblocks, shown in fig. 5.5. For $M=7$, a detail of the phase diagram at the multicritical point is provided in fig. 5.6. Again, as in the

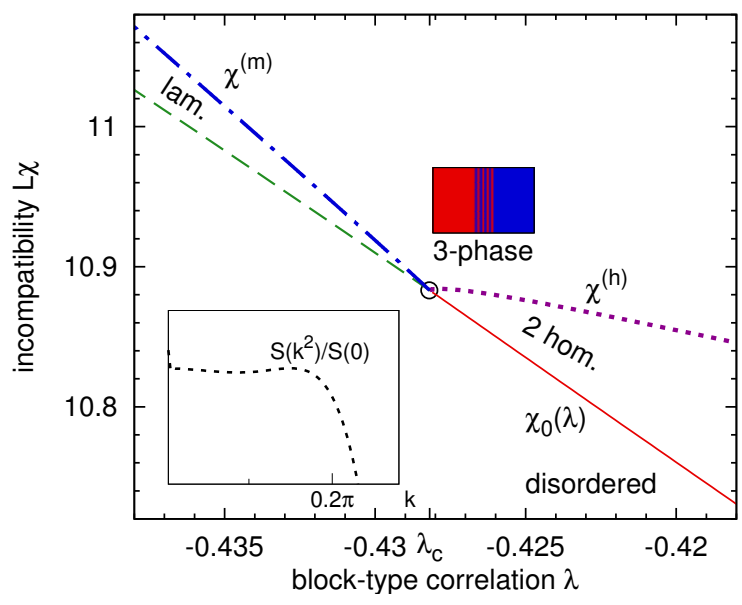

Figure 5.6: Detail of the phase diagram for triblocks with $M=7$ around the multicritical (non-Lifshitz) point $\left(\lambda_{c}, L \chi_{c}\right)=(-0.42819,10.883)$. At $\left(\lambda_{c}, L \chi_{c}\right)$, the lamellar wave number, again rescaled with $R_{\text {chain }}$, has the nonzero value $0.150 \pi$. Note that the shape of the line $\chi^{(\mathrm{h})}(\lambda)$ still differs from the shape of $\chi^{(\mathrm{h})}(\lambda)$ in the continuous-chain limit, cf. fig. 5.8 below. 
case $M<7$, for $\lambda>\lambda_{c}$ and increasing $L \chi$ in the two homogeneous phases, the dotted line $\chi^{(\mathrm{h})}$ marks the emergence of a lamellar shadow in addition to the homogeneous bi-phase cloud. Starting from a point on $\chi^{(\mathrm{h})}$, the lamellar volume fraction grows both with increasing $L \chi$ and with decreasing $\lambda$. Upon decreasing $\lambda$, the lamellar phase takes over to be the cloud on the dot-dashed line $\chi^{(\mathrm{m})}$, indicating in turn a fractionated homogeneous bi-phase shadow.

In comparison to the case $M<7$, see fig. 5.1, the three-phase coexistence region is found to be larger, again restricting the predictions to values of $L \chi$ that do not exceed considerably those of the ODT. The multicritical point is not only located at a smaller critical block correlation $\lambda_{c}$ and a higher incompatibility, but is also qualitatively different: As discussed in sec. 3.3.2, the wave number of the global instability of a disordered triblock melt with $\lambda$-distribution is discontinuous at $\lambda_{c}$ for $M \geq 7$. Thus

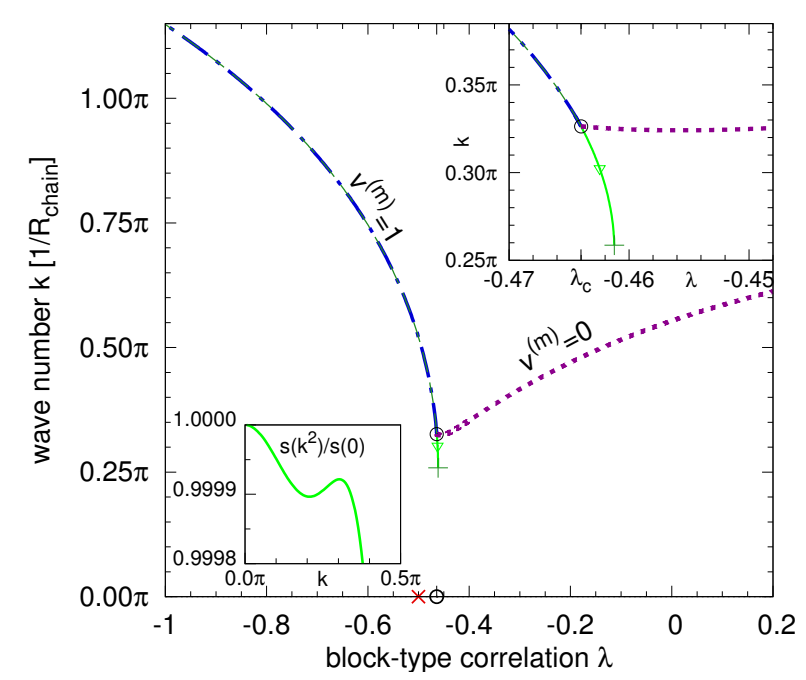

Figure 5.7: Lamellar wave number for continuous-chain triblocks. Dotdashed, dashed (blue, green) line: global lamellar phase and lamellar cloud $\left(v^{(\mathrm{m})}=1\right)$ at $\lambda<\lambda_{c}$; dotted (purple) line: lamellar shadow $\left(v^{(\mathrm{m})}=0\right)$ at $\lambda>\lambda_{c}$; solid (lightgreen) line: metastable global lamellae due to a second peak of $s\left(k^{2}\right)$, shown at $\lambda=-0.4625$ (triangle) in the bottom inset. Circles mark the wave numbers of the coexisting states at the multicritical point, crosses $(\times,+)$ indicate the end points of the lines of metastable, global phase separations, cf. fig. 5.5

when reaching $\lambda_{c}$ from above, the morphology of the global ordered state changes from two homogeneous phases (critical wave number $k_{0}=0$ ) to one lamellar phase with finite wave number $k_{0, c}$. This feature is revealed in more detail in the plot of lamellar wave numbers in fig. 5.7. At the multicritical point, both the wave number of the global instability and the wave number of lamellae in a fractionated subsystem as a function of $\Delta \lambda$ tend to the nonzero value $k_{0, c}=0.326 \pi$. Due to the two peaks of the global structure function $s\left(k^{2}\right)$ near multicriticality (see the bottom inset in fig. 5.7), metastable global lamellae occur in a small range of block-type correlations, $-0.46123 \geq \lambda>\lambda_{c}$, where the free-energy functional's absolute minimum indicates global macroscopic phase separation. Conversely, global macroscopic phase separation persists as a metastable state for $-0.5<\lambda<\lambda_{c}$ (at $\lambda=-0.5$, the second derivative of $s\left(k^{2}\right)$ at $k=0$ changes sign according to eq. (3.39) $)$. The metastable lines, whose end points are hardly resolvable in fig. 5.5 , appear in fig. 5.8 as continuations of the two parts of the line $\chi_{0}(\lambda)$ which indicate the stable structures emerging from the disordered state. Possibly due to the intersection of these metastable continuations, we find for $\lambda<\lambda_{c}$ that the transition line $\chi_{0}(\lambda)$ from the disordered to the global lamellar state 


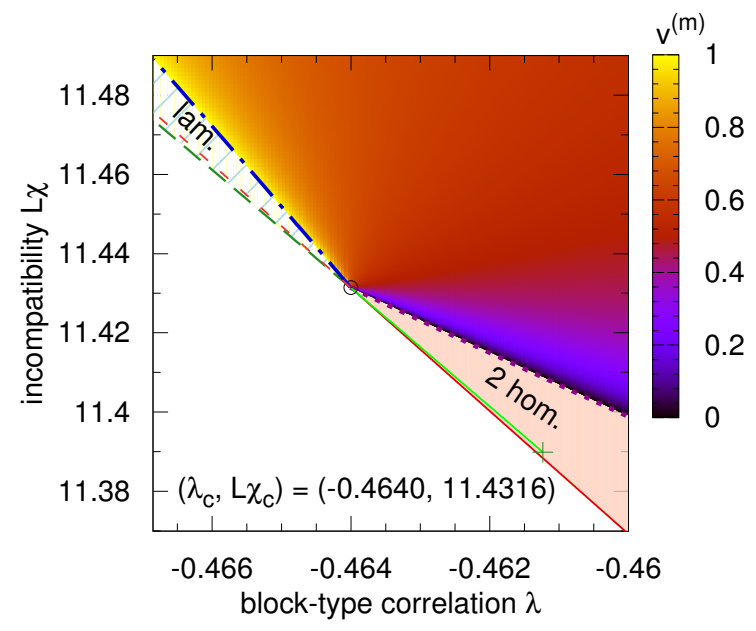

Figure 5.8: Blow-up of the phase diagram for continuouschain triblocks from fig. 5.5 around the multicritical point $\left(\lambda_{c}, L \chi_{c}\right)$, with volume fraction of the lamellar phase. Additional thin lines correspond to the metastable transitions to global states, as indicated in figs. 5.5 and 5.7 solid (green): lamellae at $\lambda>\lambda_{c}$; dashed (red): homogeneous phases at $\lambda<\lambda_{c}$.

and the lamellar cloud transition line $\chi^{(\mathrm{m})}(\lambda)$ to three-phase coexistence differ in their slopes at multicriticality, in contrast to the behavior at the Lifshitz points for $M<7$. A map of the lamellar volume fraction around the multicritical point is shown in fig. 5.8 . too (note the zoom to an even smaller region than in fig. 5.3). Upon increasing $L \chi$ from the transition line $\chi^{(\mathrm{h})}$ at $\lambda>\lambda_{c}$, the lamellar volume fraction grows rapidly to reach a nearly constant value of about 0.6 .

\section{Enhanced theory with wave-number dependence of fourth order}

In this section, we refine the analysis of the fractionation scenario by accounting for the wave-number dependence of the fourth-order coefficients of eq. (3.25), which lead to the enhanced expression eq. (3.54) for the lamellar free energy instead of the simplified version eq. (3.50). Figure 5.9 shows the results of this more detailed calculation for

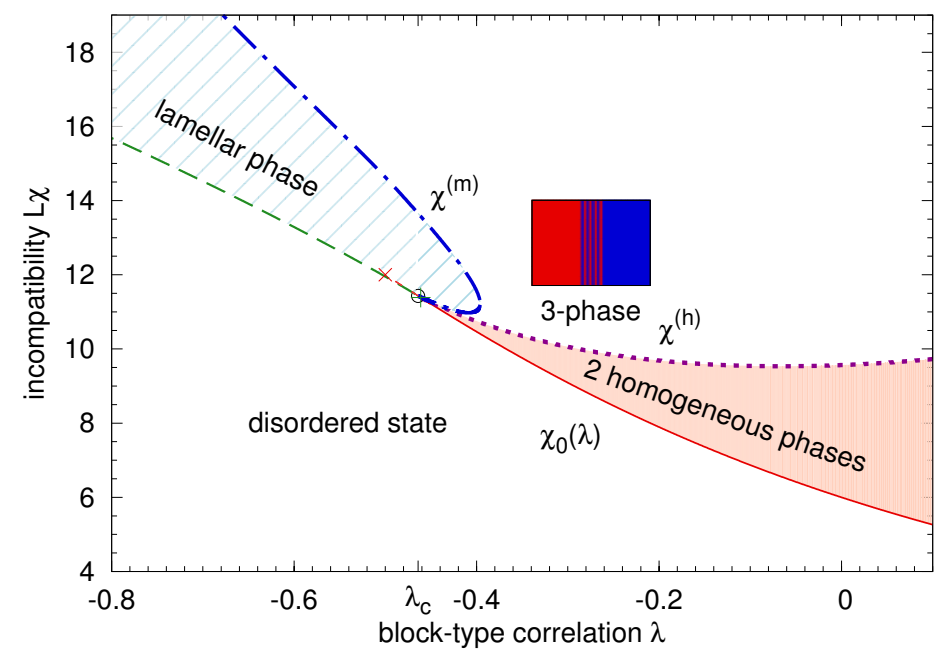

Figure 5.9: Phase diagram for continuous-chain triblocks, with restored $k$-dependence of fourth-order coefficients of eq. (3.25) in the lamellar free energy, cf. eq. (3.54). Line styles as in fig. 5.1 Crosses indicate the end points of the line of metastable macroscopic phase separations for $\lambda<\lambda_{c}(\times)$ and of metastable lamellae for $\lambda>\lambda_{c}(+)$.

the phase diagram of continuous-chain triblocks. First, we find the region of global lamellar phase separation to be larger than in fig. 5.5 and, in marked contrast to the 
results we could obtain with the simplified free energy, to extend into the half-plane $\lambda>\lambda_{c}$. The main virtue of the expression eq. (3.54) is precisely to open the possibility of global lamellae also at $\lambda>\lambda_{c}$, since the optimal wave number $k_{\text {opt }}$, cf. eq. (3.53), is not fixed by the global sequence distribution as $k_{\mathrm{m}}$, but may change upon increasing $L \chi$. Second, the transition line $\chi^{(\mathrm{h})}$ between global macroscopic phase separation and the three-phase region at $\lambda>\lambda_{c}$ is located at lower $L \chi$ than the line calculated with the simplified free energy, cf. fig. 5.5. In effect, the enhanced description of the lamellar subsystem in our fractionation scheme, which rests on a superposition of free energies, is seen to increase the tendency toward lamellar phase separation.

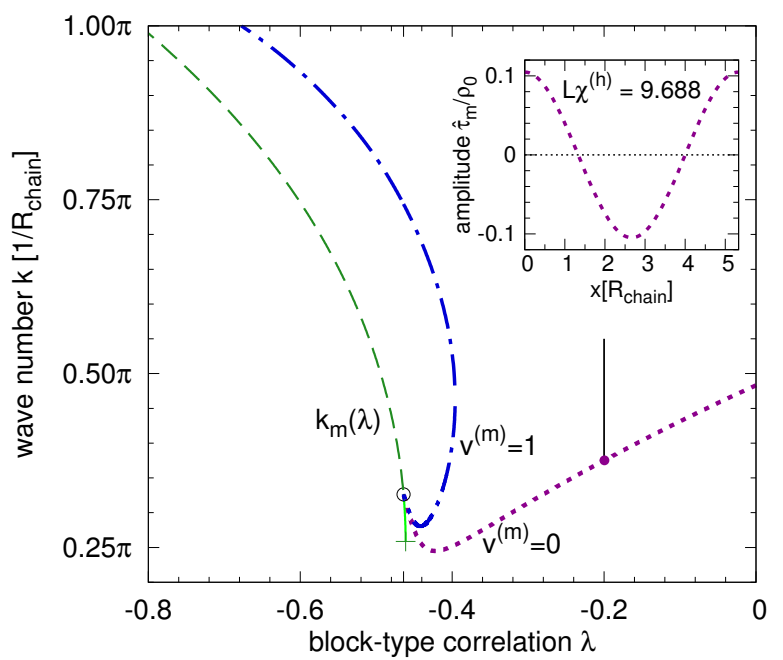

Figure 5.10: Wave number $k_{\mathrm{m}}(\lambda)$ of global lamellar instability (dashed), of lamellar cloud with $v^{(\mathrm{m})}=1$ (dotdashed), and of lamellar shadow with $v^{(\mathrm{m})}=0$ (dotted); top right inset: order-parameter amplitude in lamellar shadow at $L \chi^{(\mathrm{h})}(\lambda=-0.2)=9.688$.

The graph of the wave numbers in fig. 5.10 suggests that on the lamellar shadow line $\left(v^{(\mathrm{m})}=0, \lambda>\lambda_{c}\right)$, smaller wave numbers are realized than in the simplified picture, cf. fig. 5.7. As mentioned above, the corresponding incompatibilities $\chi^{(\mathrm{h})}$ are smaller, too, and accordingly, the amplitudes on this line. Indeed, the inset shows a lamellar shadow amplitude, which is still reasonably small at $\lambda=-0.2$, a value not in the ultimate vicinity of the multicritical point. The line $\chi^{(\mathrm{h})}$ therefore may be regarded as consistent with the expansion in the order parameter, $L \chi \hat{\tau} / \varrho_{0}$, assumed for the lamellar phase, cf. eq. 3.25), not only in the closest vicinity of $\left(\lambda_{c}, L \chi_{c}\right)$. Moreover, on the line $\chi^{(\mathrm{h})}$, the cloud is the homogeneous bi-phase, whose amplitude is calculated exactly.

At a given $\lambda<\lambda_{c}$, however, the global lamellar wave number is found to increase substantially in the range $\chi_{0}<\chi<\chi^{(\mathrm{m})}$. Due to the rather large lamellar amplitudes on the part of the line $\chi^{(\mathrm{m})}$ at $\lambda<\lambda_{c}$, the validity of the predictions for this part of $\chi^{(\mathrm{m})}$ is restricted.

The region of global lamellar phase separation at $\lambda>\lambda_{c}$ is in fig. 5.9 bounded toward larger $L \chi$ by the line $\chi^{(\mathrm{m})}$, which indicates a reentrance into the three-phase coexistence. Numerical SCFT results, shown in fig. 5.16 below, predict instead that the lamellar cloud line lies entirely in the half-plane $\lambda>\lambda_{c}$ and thus global lamellar phase separation is always favored at sufficiently large incompatibilities. 


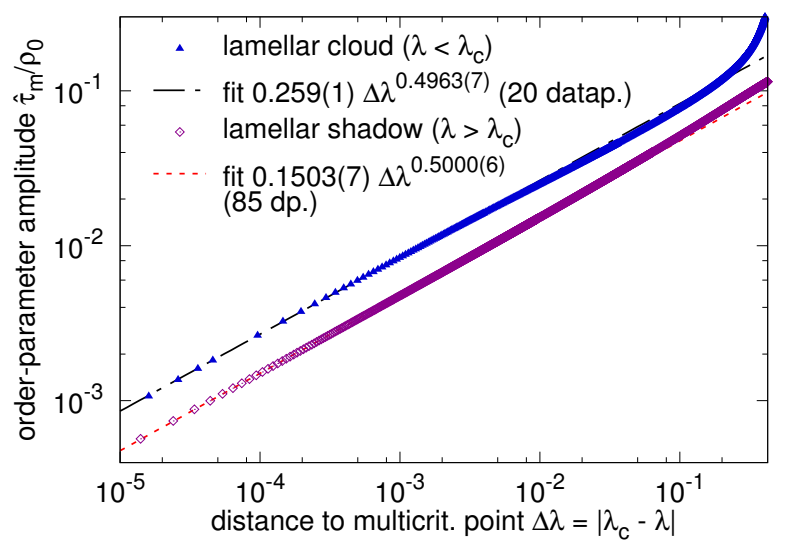

Figure 5.11: Scaling of the lamellar order-parameter amplitude on approach to the multicritical point along two three-phase transition lines for continuous-chain triblocks, with the simplified expression eq. (3.50) for the lamellar free energy.

\subsubsection{Scaling of the order-parameter amplitude at the multicritical point}

Within our simplified description of the systems with $M \geq 7$, both transition lines to three-phase coexistence, $\chi^{(\mathrm{h})}$ and $\chi^{(\mathrm{m})}$, are single-valued as a function of $\lambda$ around the multicritical point (despite the discontinuity of the wave number $k_{0}$ at the critical correlation $\lambda_{c}$ ). In this case, the numerical results along both lines $\chi^{(\mathrm{h})}$ and $\chi^{(\mathrm{m})}$ can be used to determine the critical exponent $\psi$ for the decay of the order-parameter amplitude (see fig. 5.11). Evaluating the amplitude of the lamellar cloud and shadow, respectively, we find $\psi=0.5$ along both lines consistently. In order to validate this

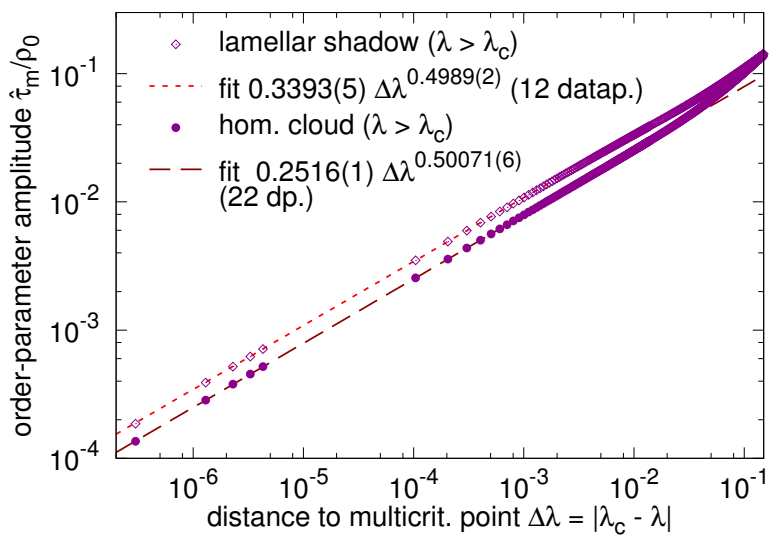

Figure 5.12: Order-parameter amplitude of homogeneous bi-phase cloud and of lamellar shadow on the line $\chi^{(\mathrm{h})}$ for continuous-chain triblocks, with enhanced expression for the lamellar free energy, cf. fig. 5.9.

result, we analyze in fig. 5.12 the scaling of the order-parameter amplitude on approach to the multicritical point for the same system, but with the enhanced theory, which had led to a different location of the transition lines, cf. figs. 5.9 and 5.5. The amplitudes, in this case evaluated on the line $\chi^{(\mathrm{h})}$, of both lamellar shadow and homogeneous bi-phase cloud vanish for $\lambda \downarrow \lambda_{c}$ with an exponent of $\psi=0.5$, which corroborates the findings for continuous-chain triblocks with the simplified theory. We conclude that the critical exponent of the order-parameter amplitude for triblocks with $M \geq 7$ is $\psi=0.5$, distinct from the exponent $\psi=1$ for the systems with $M<7$, cf. eq. (5.4). 


\subsection{Sequence fractionation for triblocks}

In this section, we return to a central aim of this work, viz., the analysis of the sequence fractionation or partitioning between coexisting subsystems of different morphologies. To this end, we can exploit the detailed information on the three-phase region obtained from the solution of the coexistence conditions, cf. sec. 4.4.

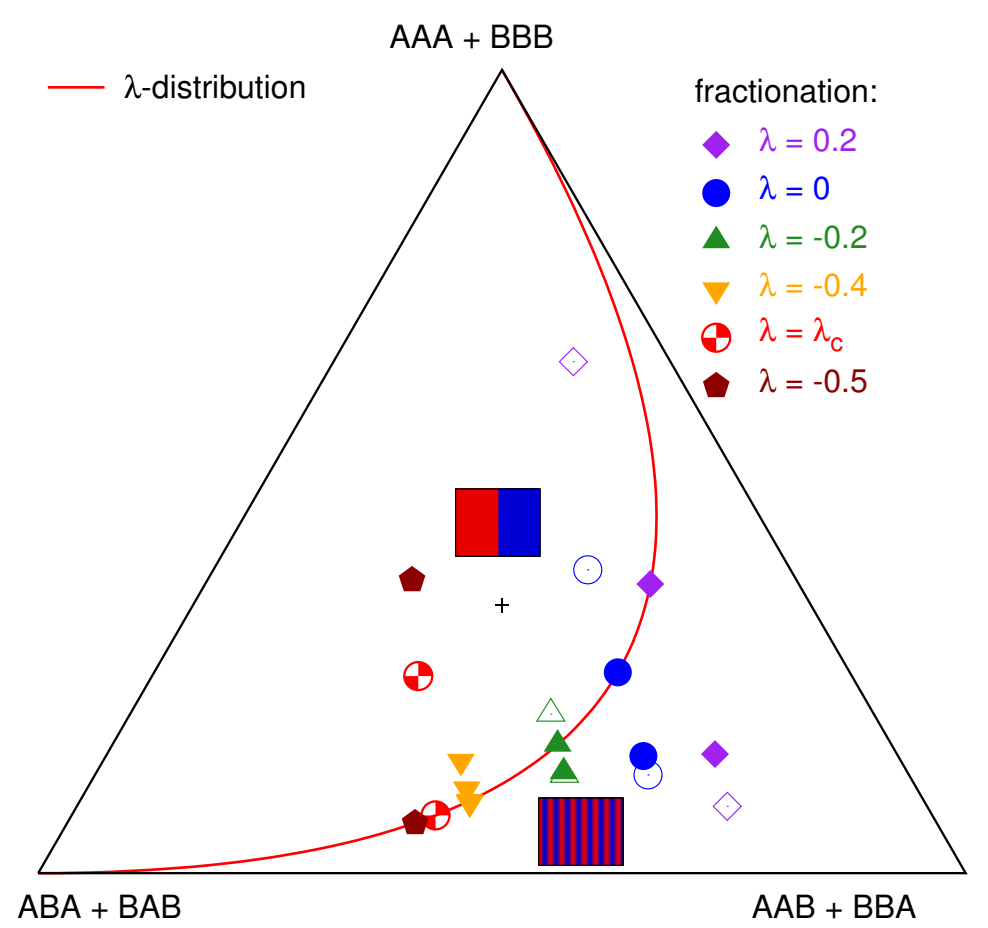

Figure 5.13: Sequence distribution triangle for random continuous-chain triblocks at various block-type correlations, with the enhanced analytical expression for the lamellar free-energy, cf. the phase diagram in fig. 5.9. The triangle is spanned by the unit vectors $\boldsymbol{n}_{\nu}$ (not drawn) pointing from the center (+, origin of the coordinate system) to the vertices, each labeled by one sequence class $\nu$. Within the triangle, one sequence distribution $\left\{p_{\nu}\right\}$ is represented as a point. The vector $\boldsymbol{p}$ of this point relative to + determines each concentration $p_{\nu}$ via $\boldsymbol{p} \cdot \boldsymbol{n}_{\nu}=3\left(p_{\nu}-1 / 3\right) / 2$, i.e., the larger the projection of the vector $\boldsymbol{p}$ onto $\boldsymbol{n}_{\nu}$, the higher is the concentration $p_{\nu}$. The origin corresponds to equal concentrations of all sequence classes $\left(p_{\nu}=1 / 3, \nu=1,2,3\right)$. Distributions defined by $\lambda$ lie on the (red) curve, with $\lambda$ ranging from -1 at the triangle's bottom left vertex to +1 at its top. Solid symbols on this curve mark the sequence distribution of the cloud phase(s) at the boundary line(s) of three-phase coexistence. Off-curve solid symbols mark the distributions of the coexisting shadow phases. Open symbols display the distributions of the coexisting subsystems at equal volume fractions $\left(v^{(\mathrm{m})}=0.5\right)$.

For triblocks, the optimization of the subsystems' distributions proceeds via selection out of three sequence classes, cf. eq. (3.42). Therefore, a distribution triangle allows for a compact map of coexisting distributions in systems characterized by different values of $\lambda$ within one diagram. In this way, fig. 5.13 depicts sequence fractionation starting from several global distributions of continuous-chain triblocks. The data 
have been obtained with the enhanced theory for the lamellar subsystem, cf. fig. 5.9. Each vertex of the triangle represents one of the sequence classes, one point within the triangle one $\mathrm{A} \leftrightharpoons \mathrm{B}$ exchange-symmetric sequence distribution (for details of the encoding see the figure caption). The sets for three supercritical values of the block-type correlation, $\lambda>\lambda_{c}, \lambda=0.2$ (diamonds), $\lambda=0$ (circles), and $\lambda=-0.2$ (up triangles), visualize the following fractionation mechanism: On the curve of $\lambda$-distributions, the solid symbol marks the sequence distribution of the homogeneous cloud phase(s) on the boundary $\chi^{(\mathrm{h})}$ of fractionated three-phase coexistence. A solid symbol of the same shape and color to the bottom right of the curve marks the distribution of the coexisting lamellar shadow phase (with zero volume fraction). The finite deviation of the lamellar shadow's sequence distribution from the $\lambda$-distribution indicates that the transition to three-phase coexistence is discontinuous. Upon increasing incompatibility, the lamellar phase's volume fraction increases (cf. fig. 5.8), and its sequence distribution departs ever more from the $\lambda$-distribution (the open symbols to the bottom right of the $\lambda$-curve display lamellae at 0.5 volume fraction). Sequence class $2(A A B / B B A)$ substantially accumulates in the lamellar phase, also class $3(A B A / B A B)$. Moreover, since the ratio of these two sequence concentrations differs from the $\lambda$-defined ratio $p_{2}(\lambda) / p_{3}(\lambda)$, the fractionated sequence distribution in the lamellar phase does not ensue from merely expelling homopolymers into the coexisting homogeneous phases at a constant concentration ratio of the two other sequence classes. (The shifted concentration ratios become visible in the detailed distribution chart for the case $\lambda=0$, in fig. 5.14.) As the volume fraction of the homogeneous, initial cloud phase(s) decreases, their distribution (at volume fraction 0.5 marked by open symbols to the top left) deviates increasingly from the $\lambda$-curve, showing in turn a particular depletion in $A A B / B B A$ sequences.

For $\lambda=-0.4$, the reentrant behavior of the three-phase boundary line, cf. fig. 5.9 . gives rise to two sets of coexisting distributions (down triangles). Upon increasing $L \chi$ in the two homogeneous phases, the three-phase region appears with a lamellar shadow (nearly on the curve of $\lambda$-distributions, shift to the bottom hardly visible), which grows with $L \chi$ in volume fraction until it becomes a lamellar cloud (now the symbol on the curve of $\lambda$-distributions) coexisting with two homogeneous shadows (triangle shifted slightly to the top). The homogeneous shadows are enriched in homopolymers and depleted from alternating sequences. Upon further increasing $L \chi$, the global lamellar phase gives way to a three-phase coexistence again. The topmost triangle represents the distribution of the homogeneous bi-phase shadow at this reentrance. For $\lambda \leq \lambda_{c}$, the lamellar cloud line is the only three-phase boundary. The topmost symbols for the critical and subcritical correlations $\lambda=\lambda_{c}$ and $\lambda=-0.5$ show the distributions of the coexisting homogeneous shadows which deviate markedly from the $\lambda$-distributions.

A detailed sequence distribution diagram for the three-phase coexistence at $\lambda=0$ is displayed in fig. 5.14. The representation of all six species' concentrations additionally visualizes the segregation within a sequence class into $A$ - and $B$-rich subspecies between the two homogeneous phases. Using again the two homogeneous phases' $A \leftrightharpoons B$ exchange symmetry in a globally symmetric distribution, only the distribution of the $A$-rich, homogeneous phase is shown; the chart for the $B$-rich phase is obtained by exchanging letters $A$ and $B$ in the key. Consistent with fig. 5.13, fractionation is seen to be effective already on the boundary $\chi^{(\mathrm{h})}$ : The sequence class concentrations of the emerging lamellae can be immediately distinguished from the $\lambda$-distribution 


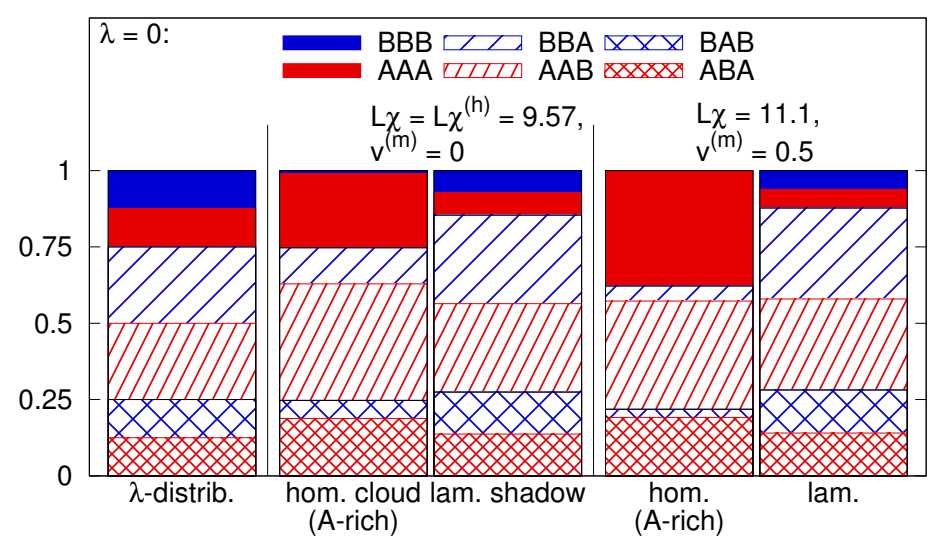

Figure 5.14: Detailed sequence distributions of the coexisting phases at $\lambda=0$ with enhanced theory (sec. 5.2), cf. fig. 5.13 Leftmost chart: $\lambda$-distribution of the disordered melt. Pairs of charts: distributions of the $A$-rich, homogeneous and of the lamellar phase; left: on the boundary $\chi^{(\mathrm{h})}$ of three-phase coexistence; right: at a lamellar volume fraction of $v^{(\mathrm{m})}=0.5$.

of the cloud. The chart for a lamellar volume fraction $v^{(\mathrm{m})}=0.5$ shows that the distribution of the lamellar subsystem does not change substantially with increasing $L \chi$. However, the increasing volume fraction of the lamellar subsystem efficiently depletes the homogeneous phases, the former cloud, from alternating sequences. Along this route, the homogeneous phases achieve to increase their amplitude.

\subsection{Three-phase coexistence for random symmetric diblocks}

Single-component diblock copolymers have been investigated in a vast number of studies both experimentally and theoretically [72, 76, 81]. The phase behavior of ternary mixture of symmetric $A B$ diblocks with equal amounts of $A$ and $B$ homopolymers, referred to as random symmetric diblock copolymers in our context, has received considerable attention, too [18, 69, 70]. Even the coexistence of subsystems with different morphologies and homopolymer concentrations has been already reported [88. Detailed, yet partially contradicting results on the phase behavior of this system are thus available, some of them with account for order-parameter fluctuations [90]. Nonetheless, we analyze the random symmetric diblock system here, in order to test both the consistency of our fractionation ansatz and its ability to predict a coexistence of different morphologies in the most simple case.

For diblocks, inspection of the global $S\left(k^{2}\right)$, cf. eqs. 3.37) and (3.39), yields the Lifshitz behavior at $\left(\lambda_{c}, \chi_{c}\right)$ for any number $M$ of segments per block, which allows us to restrict the discussion to continuous-chain diblocks. The calculations have been performed with the enhanced theory for the lamellar free energy from sec. 3.4.2. As depicted in fig. 5.15 , we can identify for the diblock system a large three-phase coexistence region in the $\lambda-\chi$ plane. However, it is central to observe that this three-phase coexistence is qualitatively different from the fractionated coexistence in the triblock system we considered before, due to the extremely low sequence 'polydispersity' of the symmetric diblock system. Only one sequence class concentration can be varied independently in this system, 


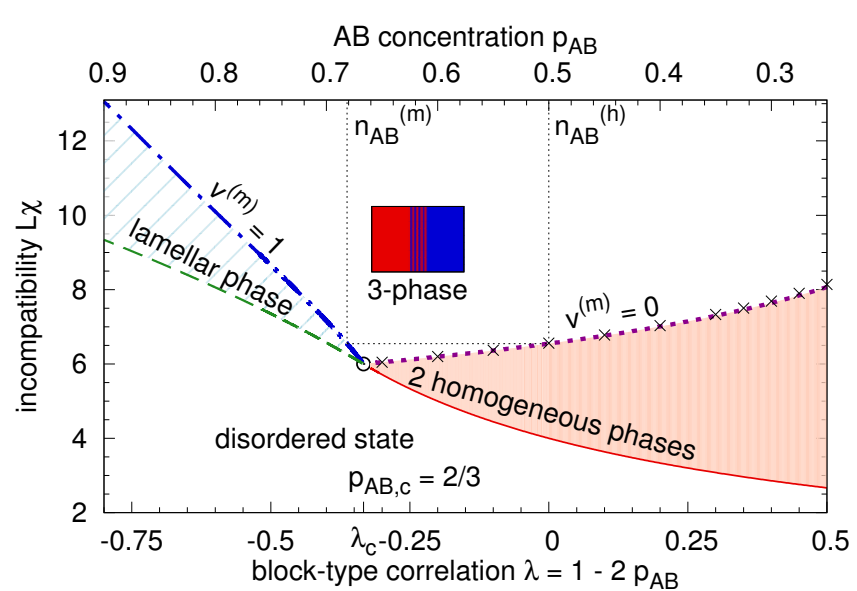

Figure 5.15: Phase diagram for random symmetric, continuouschain diblock copolymers. Line styles as in fig. 5.1. Crosses indicate the (metastable) transitions from two to three homogeneous phases predicted by multicomponent theory.

e.g., that of $A B$ chains, $p_{A B}=(1-\lambda) / 2$, such that all possible distributions are parameterized by the block-type correlation $\lambda$. Hence for any partitioning, all coexisting subsystems must have Markovian distributions confined to the plane of fig. 5.15, and sequence fractionation in the sense of non-Markovian distributions in subsystems is barred. Not visible in fig. 5.15, upon changing the global $A B$ concentration at a given incompatibility, the $A B$ concentration $n_{A B}^{(\mathrm{m})}$ in the lamellar subsystem remains constant within the three-phase region, as well as $n_{A B}^{(\mathrm{h})}$ in the two homogeneous phases. The dotted, vertical lines in fig. 5.15 indicate the concentrations $n_{A B}^{(\mathrm{m})}$, respectively $n_{A B}^{(\mathrm{h})}$, of coexisting lamellae and the two homogeneous phases at $L \chi=6.55$. When varying the global $A B$ concentration along the horizontal line connecting these lines, only the volume fraction of the coexisting subsystems changes. At a fixed incompatibility $L \chi$, we can identify the region of $A B$ contents where we find a three-phase coexistence with a "mixing gap". A global, ordered phase is not stable in this region, analogous to the density range in the liquid-gas phase transition.

By computing the instability of the two homogeneous phases at $\lambda>\lambda_{c}$ within multicomponent theory, cf. sec. 3.5 and appendix E.1. we find metastable transitions to three homogeneous phases at the incompatibilities marked by crosses in fig. 5.15. These transitions occur at values of $L \chi$ very close to, but slightly above the lamellar shadow line $v^{(\mathrm{m})}$. Indeed, the third homogeneous phase is predicted by multicomponent theory to set in with zero volume fraction as does the lamellar shadow, and with almost equal $A B$ concentration. This is yet another consistency test for our fractionation scheme. As may be expected, the free-energy density $f_{\text {frac }}$ of the three-phase coexistence including a lamellar phase is lower than that of the hypothetical three homogeneous phases. 


\subsection{Random continuous-chain triblocks analyzed with numerical SCFT}

In large parts of this thesis, Markovian, $A \leftrightharpoons B$ exchange-symmetric distributions of triblock copolymers, $Q=3$, serve as prototype of the systems which can undergo nontrivial sequence fractionation in the sense that the distributions of the subsystems deviate both quantitatively and qualitatively from the global distribution. In the study [59], we have chosen the triblock distributions not only due to their analytical tractability, but also seeking to enable a comparison with the results for continuous-chain triblocks from a complementary mean-field approach, viz., numerical self-consistent field theory (SCFT) [57, 81, 39]. A description of the numerical SCFT calculations performed by M. Müller can be found in [59], too, as well as a brief account of the underlying theory. An overview of the phase behavior of continuous-chain triblock copolymers obtained with numerical SCFT shall be given here.

\subsubsection{Three-phase coexistence lines}

In fig. 5.16, we present the boundary lines of three-phase coexistence, determined from numerical SCFT (left) and from the analytical approach (right). First, we note that
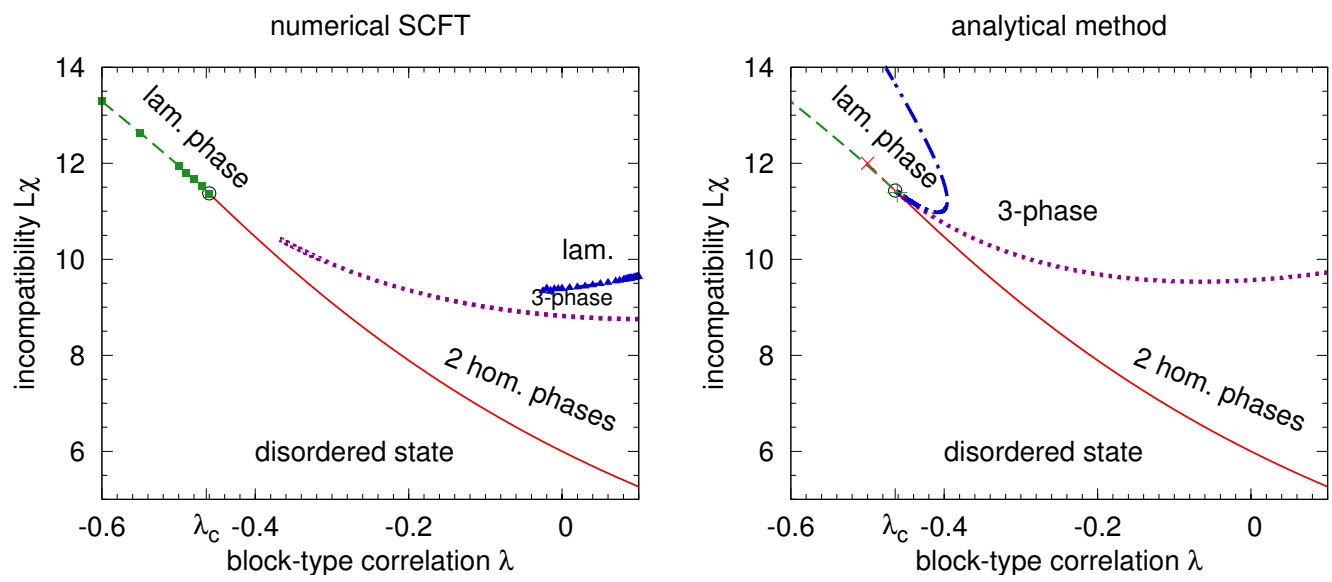

Figure 5.16: Phase diagram for random continuous-chain triblocks, calculated with numerical SCFT (left) and with analytical approach (right). Instabilities of the disordered state: solid (red): macroscopic phase separation; dashed (green): ODT. Dotted (purple): homogeneous bi-phase cloud boundary $\chi^{(\mathrm{h})}$, dot-dashed (blue): lamellar cloud boundary $\chi^{(\mathrm{m})}$ of three-phase coexistence. (On the ODT line and on $\chi^{(\mathrm{m})}$, the location of SCFT data points is additionally indicated by symbols.)

beyond the mean-field approximation, the analytical approach and SCFT have different additional limitations, such that their results for the location of three-phase boundaries are complementary. The most prominent feature of the SCFT results is the location of the entire three-phase coexistence region in the half-plane $\lambda>\lambda_{c}$ of the $\lambda$ - $\chi$ diagram. Consequently, SCFT predicts the three-phase region to be significantly smaller than the analytical method, and global lamellae to occur at large incompatibilities for all $\lambda$. In the enhanced version of the analytical theory, using the lamellar free energy 
eq. (3.54), the part of the lamellar cloud boundary near the multicritical point extends into the half-plane $\lambda>\lambda_{c}$, too. In the vicinity of the multicritical point, due to small lamellar amplitudes, the results of the analytical approach become accurate. In this region, however, the free-energy differences between competing states - disordered, global lamellae, three-phase coexistence, two homogeneous phases - become minuscule, which poses numerical difficulties for the SCFT calculations. Hence, there is no regime where both approaches are simultaneously reliable, and a direct comparison is difficult. The predictions for the cloud line $\chi^{(\mathrm{h})}$ of the homogeneous phases obtained by SCFT and by the analytical method match quite well, whereas the agreement for the lamellar cloud points, on the line $\chi^{(\mathrm{m})}$, is less satisfactory. Numerical SCFT results for these points (solid triangles) do not extend below $\lambda=-0.025$ due to the mentioned subtle free-energy differences in this region which control the phase behavior. In the analytical approach, the shape of $\chi^{(\mathrm{m})}$ is bound to be more sensitive to the approximation of small lamellar amplitudes than that of the other three-phase boundary, at which the lamellar phase is the shadow and all amplitudes are smaller.

\subsubsection{Sequence fractionation}

Despite the different location of the three-phase boundaries, the sequence distributions of the coexisting subsystems, obtained by both methods, reveal the same mechanism
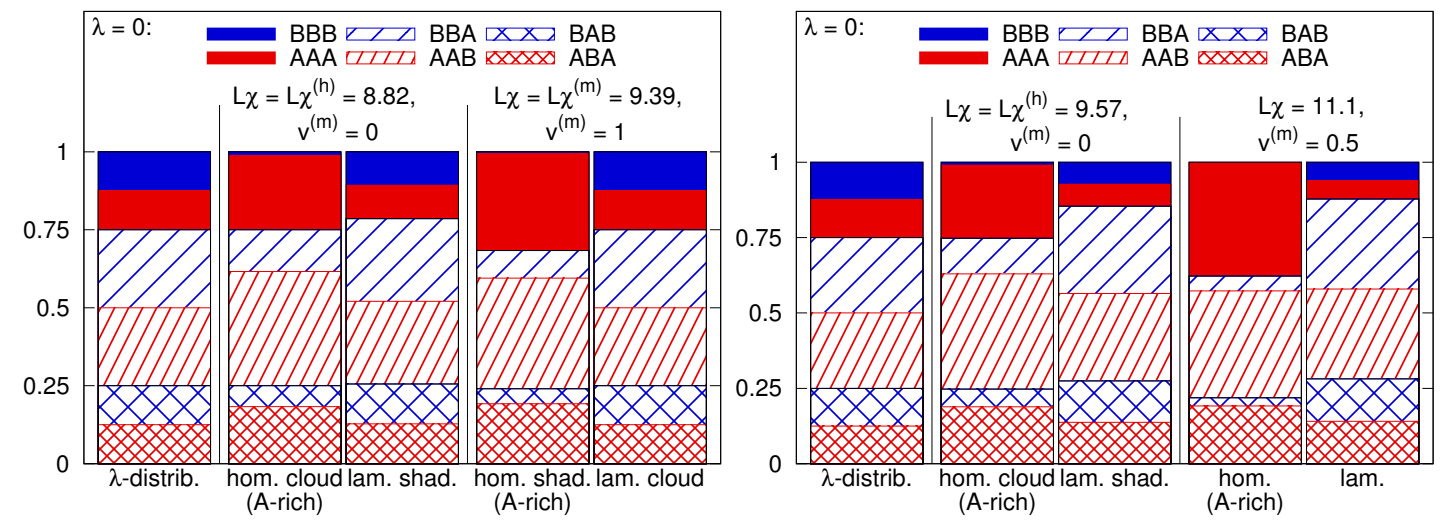

Figure 5.17: Detailed sequence distributions of the coexisting phases in random, continuouschain triblocks at $\lambda=0$ obtained with SCFT (left) and with the enhanced version of the analytical method (right), cf. fig. 5.14. Leftmost chart: $\lambda$-distribution of the disordered state and of the cloud. Pairs of charts: distributions of the $A$-rich, homogeneous and the lamellar phase; left: at the homogeneous cloud boundary; right: at the lamellar cloud boundary of three-phase coexistence (SCFT), respectively at a lamellar volume fraction of 0.5 (analytical method).

of fractionation, cf. fig. 5.17. Particularly visible is the preference of the fractionated lamellar phase for $A A B / B B A$ sequences. According to the results from the analytical method, the accumulation is more pronounced already at the onset of fractionation on the homogeneous cloud line $\chi^{(\mathrm{h})}$ of three-phase coexistence (cf. the central charts). Since the value for the incompatibility $\chi^{(\mathrm{h})}$ is higher than that obtained with SCFT, the macroscopic separation into $A$ - and $B$-rich subspecies is at a more advanced stage, too. 


\subsubsection{Discussion}

The analytical method and numerical SCFT constitute complementary approaches, which both have their benefits and limitations. In combination, they may provide a comprehensive mean-field picture of the phase behavior of random continuous-chain triblocks. For this system, both methods consistently reveal a three-phase coexistence region separating the regions of global lamellar and global homogeneous bi-phase states.

Like the analytical method, numerical SCFT invokes a mean-field approximation, but it avoids the assumptions of small order-parameter amplitudes and a single-mode description for the lamellar phase. Thus, it provides appropriate mean-field predictions for large regions of the phase diagram, but due to numerical problems fails as the multicritical point is approached and both wave numbers and free-energy differences decrease. Moreover, the applicability of numerical SCFT is severely restricted to distributions with a small number of different sequence classes, i.e., to a small number of different components.

On the one hand, our analytical mean-field approach is restricted in its validity, whenever a structured (lamellar) phase is addressed, to small lamellar order-parameter amplitudes or to the proximity of a continuous order-disorder transition. On the other hand, since we can rest on analytical expressions as far as to the formulation of the equilibrium conditions, we are able to explore accurately the vicinity of the multicritical point $\left(\lambda_{c}, \chi_{c}\right)$. Moreover, given the analytical form of the structure functions that decide on the critical behavior, we can explicitly study the influence of the model parameter $M$, the number of segments per block. 



\section{Summary and Discussion}

In this work, we have studied the equilibrium phase behavior of random symmetric $A B$ block copolymers with a fixed small number $Q$ of blocks per polymer. Our aim has been to investigate the possible coexistence of subsystems with different morphologies, here a lamellar phase and two homogeneous phases, within an analytical mean-field approach. For this purpose, we have first defined a microscopic model with a minimal set of interactions and calculated free energies of the basic phase-separated states, detailing the dependence on the sequence distribution. On this basis, we have formulated the free energy of a partitioned state with account for sequence exchange between subsystems and derived the coexistence conditions. The resulting picture of three-phase coexistence provides insight into the mechanism of sequence fractionation: Each of the coexisting phases is found to accumulate preferably those sequences that match best its morphology.

\subsection{Summary}

The model system is a melt of different $Q$-block polymer chains (sequences), generated from polymer blocks of type $A$ and $B$, following a Markov process with correlation parameter $\lambda$. Each block contains $M$ segments. Segments within one chain are bonded irreversibly, interacting via connectivity, segments on different chains experience two nonbonded interactions: incompatibility with strength $\chi \propto 1 / T$, which favors accumulation of segments of equal type and separation of segments of different types, and excluded volume interaction of strength $\kappa$, which prevents unphysical instabilities.

First, we review our way of deriving the free energy.

\section{Free-energy functional}

Starting from the Hamiltonian of this model, we have set up in chap. 3 the normalized canonical partition function $\mathcal{Z}$ including the Gibbs over-counting correction for identical chains. Although the indistinguishability of chains is irrelevant for a global state of the system, it has to be accounted for if thermodynamic equilibrium can be reached by the formation of coexisting subsystems with chain exchange. Such a coexistence can then be described transparently, without using the grand-canonical ensemble, within the canonical ensemble as well, yielding a superposition of the subsystems' free-energy functionals, cf. chap. 4, which has to be minimized with respect to volume fractions and sequence concentrations. For the evaluation of $\mathcal{Z}$, eq. 3.1), we have chosen to apply to each Fourier mode of the fields of $A$ segment excess $\left(\sigma_{\boldsymbol{k}}\right)$ and total segment density $\left(\varrho_{\boldsymbol{k}}\right)$ a Hubbard-Stratonovich (HS) transformation. This transformation decouples the fields from the inter-chain interactions and replaces terms quadratic in $\sigma$ and $\varrho$ by bilinear terms $\sigma \hat{\sigma}$ and $\varrho \hat{\varrho}$, at the cost of introducing new integration variables $\hat{\sigma}$ and $\varrho$, called interaction fields. As a result of this procedure, an effective Hamiltonian $\hat{h}$ of $\hat{\sigma}$ 
and $\hat{\varrho}$ arises, and the integrand of $\mathcal{Z}$ is expressed by products of single-chain partition functions for each sequence $\nu, \hat{z}_{\nu}[\hat{\sigma}, \hat{\varrho}]$. The latter, being integrals over single-chain conformations only, can be calculated expanding the exponential up to, in principle, any order in $\hat{\sigma}$ and $\hat{\varrho}$. In the thermodynamic limit, the integrations over $\hat{\sigma}$ and $\hat{\varrho}$ can then be performed using the saddle-point method. The saddle-point values $\hat{\tau}_{\boldsymbol{k}}$ and $\hat{\omega}_{\boldsymbol{k}}$, i.e., the averages of $\hat{\sigma}_{\boldsymbol{k}}$ and $\hat{\varrho}_{\boldsymbol{k}}$, can be shown to equal the averages of the original fields $\sigma_{\boldsymbol{k}}$, respectively $\varrho_{\boldsymbol{k}}$, which vanish in the disordered homogeneous melt for $k \neq 0$. Hence, if upon increase of the incompatibility $\chi$, the disordered state becomes unstable toward $A$ - $B$ separation at $\chi_{0}$, the amplitudes $\hat{\tau}_{\boldsymbol{k}}, \hat{\omega}_{\boldsymbol{k}}$ remain small near $\chi_{0}$ in the case of a continuous phase transition (valid for $A \leftrightharpoons B$ exchange symmetry). This enables us to derive the saddle-point equations from an expansion of the effective Hamiltonian $\hat{h}$ in $\hat{\sigma}_{\boldsymbol{k}}$ and $\hat{\varrho}_{\boldsymbol{k}}$ and to eliminate from $\hat{h}$ the density interaction field $\hat{\varrho}$. The result is an expansion solely in the field $\hat{\tau}$, the order parameter, up to fourth order, i.e., a free-energy functional $f[\hat{\tau}]$ of Landau form. The novel feature in our derivation is to use a decoupling method which naturally leads to an expansion in the interaction field, not in the original $A$ excess density, a route deviating from earlier derivations of free-energy functionals for block copolymer melts [76, 42], cf. appendix A.3. Also, the sequence heterogeneity has been addressed without resorting to the replica method.

\section{Instability and structure function}

The second-order coefficients of the free-energy functional are determined by an averaged sequence-structure function $S\left(k^{2}\right)$, which can be related to the mean-field scattering function of the melt, cf. sec. 3.3. Its maximum yields the inverse of the critical incompatibility $\chi_{0}$, the instability of the disordered state, and the maximum position $k_{0}$ determines the mode $\hat{\tau}_{k_{0}}$ whose second-order coefficient vanishes at $\chi=\chi_{0}$. The structure function $S\left(k^{2}\right)$ has been calculated analytically in terms of the sequence probabilities $p_{\nu}$ and the functions for individual sequences $\nu$, particularly also for Markovian distributions with correlation parameter $\lambda$. For triblock copolymers, $S\left(\lambda, k^{2}\right)$ turns out to depend critically on the number $M$ of segments per block:

1. $M<7$ : For $\lambda$ above a critical $\lambda_{c}$, the maximum of $S\left(\lambda, k^{2}\right)$ is located at $k_{0}=0$, indicating macroscopic phase separation at $\chi=\chi_{0}(\lambda)$ into two homogeneous, $A$ respectively $B$-rich, phases. Upon decreasing $\lambda$ below $\lambda_{c}$, the maximum position $k_{0}$ of $S\left(\lambda, k^{2}\right)$ increases continuously from zero, $k_{\mathrm{m}}>0$ indicating an instability of the disordered melt toward a lamellar $A$ excess variation with wave number $k_{\mathrm{m}}$. The continuous increase of $k_{0}$ identifies the multicritical point $\left(\lambda_{c}, \chi_{c}\right)$, with $\chi_{c}=\chi_{0}\left(\lambda_{c}\right)$, as a Lifshitz point.

2. $M \geq 7:$ As in the case $M<7$, for large $\lambda$, the maximum position of $S\left(\lambda, k^{2}\right)$ is $k_{0}=0$, but upon decreasing $\lambda, S\left(\lambda, k^{2}\right)$ develops a second maximum at a wave number $k_{\mathrm{m}}>0$. The second maximum (corresponding to a metastable lamellar phase) reaches equal height as the maximum at $k_{0}=0$ at a correlation $\lambda_{c}$ which depends on $M$. Thus, at $\chi_{c}=\chi_{0}\left(\lambda_{c}\right)$, the lamellar instability emerges with a finite wave number $k_{\mathrm{m}}>0$ in contrast to the Lifshitz behavior. This result holds in the limit of continuous-chain triblocks, too. 
In studies on block copolymers so far, albeit mostly for the cases of either large $Q$ or $Q=2$, the multicritical point $\left(\lambda_{c}, \chi_{c}\right)$ had been assumed to be always of Lifshitz type.

The fourth-order coefficients of the free-energy functional $f[\hat{\tau}]$ contain several sequencestructure functions, which we have computed analytically for individual sequences. Due to the contributions of the density interaction field $\hat{\varrho}$, eliminated at the saddle point, the fourth-order terms are positive for a sufficiently large compression modulus, guaranteeing a nonzero, but finite amplitude $\hat{\tau}_{\mathrm{m}}$ of an ordered state not too far from the instability $\chi_{0}$. Keeping for a lamellar structure the contribution of the dominant mode with wave number $k_{\mathrm{m}}$ (respectively $k_{\text {opt }}$, which varies with $\chi$ in the enhanced theory, cf. sec. 3.4.2) only, minimization of $f[\hat{\tau}]$ with respect to the amplitude gives the free energy $f_{\mathrm{m}}$ of a lamellar state.

The free energy $f_{\mathrm{h}}$ of a homogeneous bi-phase state (generally, of multiple homogeneous phases) has been obtained using a description with component densities and again applying a HS transformation, cf. sec. 3.5. Since an expansion is not necessary in this case, the result is valid for arbitrary amplitudes.

In both $f_{\mathrm{m}}$ and $f_{\mathrm{h}}$, we have specified the dependence on the sequence concentrations.

\section{Fractionated three-phase coexistence}

The analytical expressions for the free energies $f_{\mathrm{m}}$ and $f_{\mathrm{h}}$ of lamellae, respectively homogeneous bi-phase, have been employed in a superposition ansatz

$$
f_{\text {frac }}=v f_{\mathrm{m}}+(1-v) f_{\mathrm{h}}
$$

for the free energy of a possible three-phase state, with yet undetermined sequence concentrations and volume fraction $v$. The constraint of fixed global $\lambda$-defined concentrations has been incorporated via chemical potentials acting as Lagrange multipliers. Subsequently, we have minimized the free-energy function $f_{\text {frac }}$ with respect to the independent parameters, in the case of triblocks, the volume fraction $v$ of one subsystem and two concentrations. With analytically computed partial derivatives of $f_{\text {frac }}$, we have arrived at a set of equilibrium conditions which has been solved numerically for the parameters.

Indeed, there is a region in the $\lambda-\chi$ plane, in which the minimized free energy density $f_{\text {frac }}(\lambda, \chi)$ results to be smaller than that of the $\lambda$-defined global ordered (lamellar or bi-phase) state. This implies the existence of a third ordered state, viz., three-phase coexistence, which destabilizes the known ordered states at the boundary lines of the detected region, $\chi^{(\mathrm{h})}(\lambda)$ toward the region of global bi-phase states and $\chi^{(\mathrm{m})}(\lambda)$ toward the region of global lamellar states. These lines meet at the multicritical point $\left(\lambda_{c}, \chi_{c}\right)$ and separate the region of global lamellar states from the region of global bi-phase states, see, e.g., fig. 5.9.

Starting from the global homogeneous bi-phase and increasing $\chi$, at the transition $\chi^{(\mathrm{h})}$ an infinitesimal volume fraction (shadow) emerges, which displays lamellar structure with finite wave number and finite amplitude. This shadow phase accumulates those copolymer sequences, which, due to incompatibility of $A$ and $B$ blocks, match better a lamellar order than the homogeneous bi-phase order. Consequently, the sequence concentrations in this shadow phase are radically different from the concentrations in the homogeneous bi-phase cloud, see fig. 6.1, cf. also figs. 5.13 and 5.14. This process, 

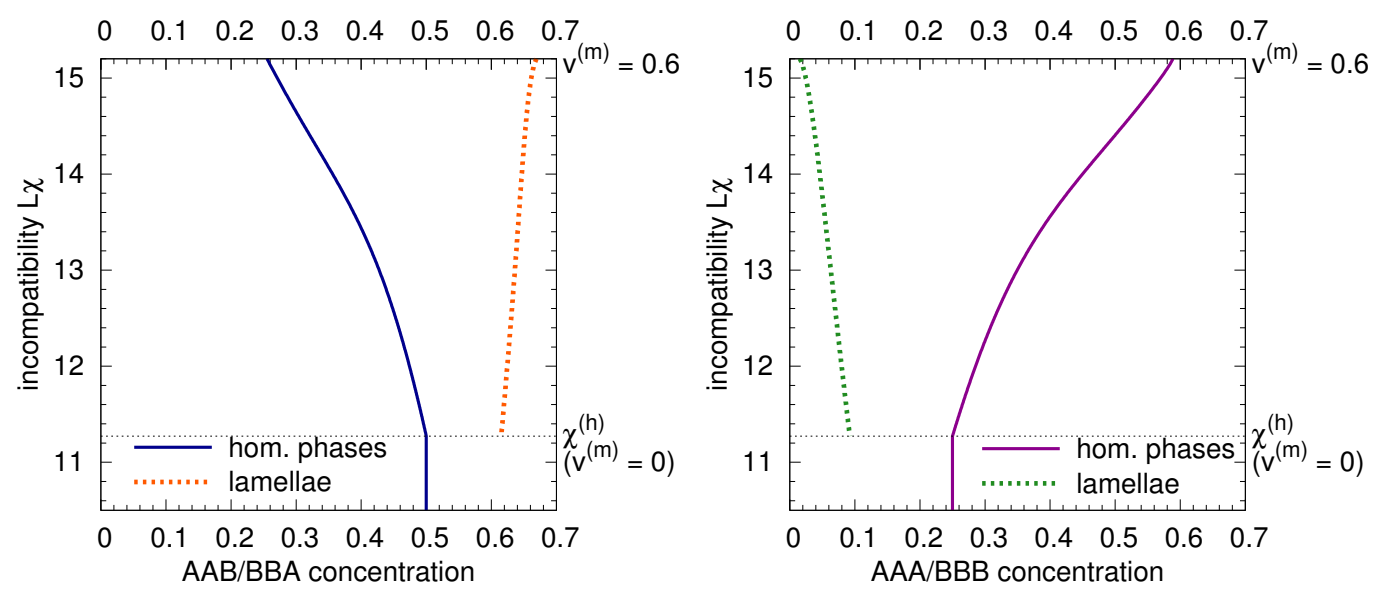

Figure 6.1: Sequence class concentrations of coexisting phases in continuous-chain Markovian triblocks at $\lambda=0$ as a function of $L \chi$, starting from the lamellar shadow line $\chi^{(\mathrm{h})}$, cf. fig. 5.5 .

exemplified for the transition line $\chi^{(\mathrm{h})}$, is called fractionation. Figure 6.1 shows the concentrations in the initial shadow phase to change only moderately upon further increasing $\chi$, suggesting that fractionation is efficient from the start. With increasing lamellar volume fraction, the coexisting homogeneous bi-phase is progressively depleted from 'mismatching' sequences.

\section{Multicritical behavior}

The model comprises a variety of systems, differing in the parameters $Q, M$, and $\lambda$. Our analytical approach has proven suited to study in detail the phase behavior in dependence on these parameters, since the global structure functions $S\left(k^{2}\right)$, respectively $s\left(k^{2}\right)$, decisive for the critical behavior, have been obtained in closed form as functions of $Q, M$, and $\lambda$, see eqs. (3.37) and (3.39). This has enabled us to explore particularly the effect of varying the parameter $M$, the number of segments per block, on the system's behavior near the multicritical point $\left(\lambda_{c}, \chi_{c}\right)$.

For Markovian symmetric triblocks $(Q=3)$, the fractionation theory corroborates that the behavior at the multicritical point depends sensitively on $M$ : For a small number of segments per block, $M<7$, the wave number of the global instability of the disordered state vanishes continuously as $\lambda \uparrow \lambda_{c}$ (Lifshitz behavior). Consistently, approaching $\left(\lambda_{c}, \chi_{c}\right)$ from the three-phase region, the wave number in the lamellar subsystem (of any volume fraction) vanishes. For more segments per block, $M \geq 7$, including the continuous-chain limit, the wave number of both global lamellae and of lamellar subsystems remains nonzero at $\left(\lambda_{c}, \chi_{c}\right)$. This implies a discontinuous change in the wave number from homogeneous to lamellar phases when passing through $\left(\lambda_{c}, \chi_{c}\right)$.

The critical dependence on $M$ is reflected also in the scaling of the order-parameter amplitude on approach to $\left(\lambda_{c}, \chi_{c}\right)$ : For the finite amplitudes on the transition lines to the three-phase region, the critical exponent is $\psi=1$ for $M<7$, and $\psi=0.5$ for $M \geq 7$. The multicritical behavior for random symmetric triblocks is summarized in table 6.1. 


\begin{tabular}{l|l|l} 
segments $M$ per block & critical exponent $\psi$ & wave number at $\left(\lambda_{c}, L \chi_{c}\right)$ \\
\hline$M<7$ & 1 & 0 \\
$M \geq 7$ & 0.5 & nonzero
\end{tabular}

Table 6.1: Critical exponents of order-parameter amplitude $\hat{\tau}$ on approach to the multicritical point $\left(\lambda_{c}, L \chi_{c}\right)$ and lamellar wave number at $\left(\lambda_{c}, L \chi_{c}\right)$ for random symmetric triblocks.

We note that the multicritical behavior for $M \geq 7$ is obviously related to the intersection at $\left(\lambda_{c}, \chi_{c}\right)$ of the global instability lines, which on both sides of $\left(\lambda_{c}, \chi_{c}\right)$ continue as transition lines to metastable, global ordered phases, cf. fig. 5.8. A reason for the discontinuity of the wave number at $\left(\lambda_{c}, \chi_{c}\right)$ in this case might be that increasing $M$ increases the effective strength of the incompatibility $\left(\propto M^{2}\right)$ between blocks, without substantial change of $L \chi_{c}$. Concomitantly, the tendency toward lamellar phase separation with finite wavelength increases.

For diblocks, $Q=2$, we find a three-phase coexistence region, too, but there is no critical dependence on $M$, especially $\left(\lambda_{c}, \chi_{c}\right)$ is a Lifshitz point for all $M$.

For $Q \in[4,5, \ldots, 9]$, preliminary studies of the global structure function $S\left(k^{2}\right)$ for various $M$, including the continuous-chain limit respectively, have not indicated a double-peak of $S\left(\lambda, k^{2}\right)$ around the conjectured critical correlation $\lambda_{c}$. A tentative conclusion is that these systems neither show a nonzero lamellar wave number at $\left(\lambda_{c}, \chi_{c}\right)$ nor metastable states in the vicinity of this point, such as detected for $Q=3$ and $M \geq 7$. However, without calculating the actual three-phase transition lines, these studies are not more than a hint, since only the value $\lambda_{c}$ of a Lifshitz point can be determined analytically from $S\left(k^{2}\right)$ or $s\left(k^{2}\right)$, cf. sec. 3.3.2. Thus, the tedious method of curve-analyzing the global structure function has to scan a considerable $\lambda$-range at each $Q$ and $M$.

\subsection{Discussion}

The route from macroscopic to structured phase separation is detected to be fundamentally different from that proposed, e.g., by Leibler et al. [76, 42, who found, with increasing $\chi$, a direct transition from a global bi-phase to a global lamellar state. According to our analysis, instead, the two regions of global ordered states are separated by a wedge of three-phase coexistence emanating from the multicritical point, see fig. 5.9 for $\lambda>\lambda_{c}$ near $\left(\lambda_{c}, \chi_{c}\right)$. (The enhanced version of the lamellar free energy, cf. eq. (3.54), allows for a global lamellar phase at $\lambda>\lambda_{c}$.) Via the free-energy function for a fractionated three-phase coexistence, ordered states of the system beyond global morphologies are taken into account, resulting in the following transition: On increasing $\chi$, a structured phase first becomes stable in a subsystem with vanishing volume fraction and with a sequence distribution different from the global one. Thereby, the system is enabled to change gradually from the homogeneous bi-phase to the lamellar state. The transition from the homogeneous bi-phase to three-phase coexistence indeed occurs at a smaller $\chi^{(\mathrm{h})}<\chi_{\mathrm{L}}$ than the direct transition to global lamellae conjectured by Leibler from the free-energy comparison of global states only. 
A fractionation mechanism similar to that driven by incompatibility in a fixed sequence distribution is found to be at work when comparing Markovian distributions with different $\lambda$, cf. fig. 5.1. Starting from a global homogeneous bi-phase and decreasing $\lambda$ continuously, the transition to a global lamellar state is not abrupt, but is initiated by the emergence of an infinitesimal volume fraction with sequence concentrations deviating from that of the homogeneous cloud. Upon further decreasing $\lambda$, the volume fraction of the shadow increases until the global lamellar state is reached.

The correlation parameter $\lambda$ determines the average length $L_{A}$ of pure $A$ subsequences for all copolymers in the system (or the length of $B$ subsequences; $L_{B}=L_{A}$ for symmetric systems). Characterizing the systems by this parameter, the region of threephase coexistence corresponds to a range of global $L_{A}$ values for which one ordered state with a single morphology is unstable. Instead, two sequence distributions, which correspond to different values $L_{A}$ and therefore allow for different ordered phases, coexist in subsystems, their volume fractions dictated by the global distribution. The range of unstable global $L_{A}$ values or distributions can be seen as the analogue of the density window of phase coexistence at a discontinuous liquid-gas transition. In general, a set of fixed chemical potentials for each sequence class, to be realized by a coupled copolymer reservoir, would play the role of constant pressure at the transition. For the 'random' diblock system, cf. sec. 5.4, we had identified the concentration $n_{A B}$ as the analogue of density; at fixed $\chi$, the concentrations $n_{A B}$ of the coexisting subsystems do not change upon varying the global $A B$ concentration, only the volume fractions.

For global macroscopic phase separation of Markovian block copolymers at $\lambda>\lambda_{c}$, the critical region $L\left(\chi-\chi_{0}\right)$, within which the mean-field approximation fails, has been estimated with the help of a Ginzburg criterion by Houdayer and Müller [62. Following this study, the Ginzburg number Gi scales as $\mathrm{Gi} \propto Q^{2} / M$, i.e., the critical region does not shrink simply with the degree of polymerization $L=Q M$, in contrast to the result of the analysis for homopolymers. Precisely for small $Q$, which have been considered here, the mean-field predictions should therefore be reliable, the reliability increasing with $M$. This validates the prediction of a nonzero lamellar wave number at multicriticality, the non-Lifshitz behavior, particularly for continuous-chain triblocks.

\subsection{Perspectives}

An obvious continuation of this work is to study the supposed three-phase coexistence and the character of the multicritical point for Markovian block copolymers with a larger number $Q$ of blocks. As regards the multicritical behavior, the closed form of the global structure function $S\left(k^{2} \mid \lambda, Q, M\right)$ for these systems should in principle allow for a systematic way to extract the possibly critical dependence on $M$, the number of segments per block.

Arbitrary sequence distributions beyond the Markovian distributions become accessible and can be analyzed with sequence-specific structure functions as computed in this thesis. Of interest in this context might be those sequences or sequence distributions whose structure gives rise to competing wave numbers and thus to metastable, ordered phases, such as observed here for Markovian triblocks with $M \geq 7$ at multicriticality.

Phase coexistence with sequence fractionation could be analyzed also for random 
symmetric, but (weakly) compressible block copolymer melts with finite $\kappa$, cf. the density interaction modes, eq. (3.24), represented in fig. 3.2.

Similarly, interesting features of the phase behavior may arise for random block copolymers without $A \leftrightharpoons B$ exchange symmetry, cf. the structure function in appendix A.2, which instead of the lamellar state display ordered morphologies such as micelles or hexagonally arranged cylinders [76, 95, 79]. The discontinuous phase transitions in this system class, however, call for a different way of computing the free energies. As mentioned earlier, the transition from the disordered to a global structured state already for symmetric systems is expected to be weakly first-order due to fluctuations [16, 40. The effects of fluctuations on the transition lines to three-phase coexistence remain to be explored, especially to address the possible existence of a structured, but disordered micro-emulsion around the multicritical point of mean-field theory.

Based on the general derivation of the effective Hamiltonian and the vertex functions within the presented mean-field framework, the description of a structured phase might be improved, e.g., by considering the sixth order in the expansion. With such a refined description, the three-phase coexistence might turn out to be shifted in favor of the structured phase.

Another generalization is to consider block copolymers built from more than two segment types.

Phase coexistence enabled by component selection in a multi-component system may be of interest not only for random block copolymers, but also for a broader class of polydisperse systems, cf., e.g., refs. [110, 33, 111]. To those systems with relatively low degree of polydispersity, the presented field-based approach with vertex functions calculated individually for each component might be applicable.

Returning to copolymers, fractionation may produce structured phases in subsystems beyond the ordered microstructured phases. Long random copolymers, for example, are expected to display frozen, random structures in coexistence with domains of macroscopic phase separation. Moreover, the influence of constraints on the occurrence of a structured phase could be studied: e.g., how does a micellar phase of diblocks, constrained at low temperatures by introducing crosslinks between different chains, behave upon increasing temperature above the order-disorder transition? Finally, nonequilibrium phase separation in heterogeneous block copolymers with dynamical pattern formation poses more challenging questions. 



\section{A Derivation of the free energy functional for structured phases}

\section{A.1 Hubbard-Stratonovich transformation}

The Hubbard-Stratonovich (HS) transformation [116, 64] originates in the idea to express an exponential quadratic in one variable by an integral of a Gaussian in another variable, e.g., for one real-valued variable $\mathrm{x}$ and $\alpha \in \mathbb{R}, \alpha>0$ :

$$
\begin{aligned}
\mathrm{e}^{\alpha x^{2}} & =\left(\frac{\alpha}{\pi}\right)^{1 / 2} \int_{\mathbb{R}} \mathrm{d} y \mathrm{e}^{-\alpha y^{2}+2 \alpha x y}, \\
\mathrm{e}^{-\alpha x^{2}} & =\left(\frac{\alpha}{\pi}\right)^{1 / 2} \int_{\mathbb{R}} \mathrm{d} y \mathrm{e}^{-\alpha y^{2}+i 2 \alpha x y} .
\end{aligned}
$$

Applied to the evaluation of partition functions, it can be employed to transform a part of the Hamiltonian quadratic in a complex field $w$ (e.g., a Fourier mode of a density) to a term linear in $w$, at the cost of introducing conjugated fields $\hat{u}$ and $\hat{v}$, which couple linearly to the real and the imaginary part of $w$ and which each have a Gaussian probability density:

$$
\begin{aligned}
\mathrm{e}^{\alpha|w|^{2}} & =\frac{\alpha}{\pi} \iint_{\mathbb{R}} \mathrm{d} \hat{u} \mathrm{~d} \hat{v} \mathrm{e}^{-\alpha\left(\hat{u}^{2}+\hat{v}^{2}\right)+2 \alpha(\hat{u} \operatorname{Re} w+\hat{v} \operatorname{Im} w)}, w, \hat{u}, \hat{v} \in \mathbb{C}, \alpha \in \mathbb{R}, \alpha>0 ; \\
\mathrm{e}^{-\alpha|w|^{2}} & =\frac{\alpha}{\pi} \iint_{\mathbb{R}} \mathrm{d} \hat{u} \mathrm{~d} \hat{v} \mathrm{e}^{-\alpha\left(\hat{u}^{2}+\hat{v}^{2}\right)+i 2 \alpha(\hat{u} \operatorname{Re} w+\hat{v} \operatorname{Im} w)} \\
& =\frac{\alpha}{\pi} \iint_{\mathbb{R}} \mathrm{d} u \mathrm{~d} v \mathrm{e}^{-\alpha\left\{(u+i \operatorname{Re} w)^{2}+(v+i \operatorname{Im} w)^{2}\right\}+i 2 \alpha\{(u+i \operatorname{Re} w) \operatorname{Re} w+(v+i \operatorname{Im} w) \operatorname{Im} w\}},
\end{aligned}
$$

$u, v \in \mathbb{R}$. (For convenience, the fields $\hat{u}$ and $\hat{v}$ may be combined into one complex field $\hat{w}:=\hat{u}+i \hat{v}$, note however, that for saddle-point evaluation, the integrations over $\hat{u}$ and $\hat{v}$ have to be extended independently to $\mathbb{C}$ in eq. (A.2b).) Rescaling the conjugated variable with an arbitrary $c \in \mathbb{R}, c>0$ gives generalizations of eq. (A.1), such as

$$
\mathrm{e}^{\alpha x^{2}}=\left(\frac{c}{\pi}\right)^{1 / 2} \int_{-\infty}^{+\infty} \mathrm{d} y \mathrm{e}^{-c y^{2}+2 \sqrt{c \alpha} x y}, \quad \alpha, c \in \mathbb{R}, \alpha, c>0 .
$$

\section{A.1.1 Saddle points and thermodynamic limit}

In eq. A.2a, the saddle-point values of $\hat{u}$ and $\hat{v}, \overline{\hat{u}}, \overline{\hat{v}}$, are simply

$$
\overline{\hat{u}}=\operatorname{Re} w \text { and } \overline{\hat{v}}=\operatorname{Im} w \text {, such that } \overline{\hat{u}}^{2}+\overline{\hat{v}}^{2}=|w|^{2} .
$$


In eq. A.2b), the saddle-point values of $\hat{u}$ and $\hat{v}$ lie on the imaginary axis, which is taken into account by the path parameterization in the second line. In this case,

$$
\overline{\hat{u}}=i \operatorname{Re} w, \overline{\hat{v}}=i \operatorname{Im} w \text {, and } \overline{\hat{u}}^{2}+\overline{\hat{v}}^{2}=-|w|^{2} .
$$

Per definition, the variance of a conjugated field with respect to its saddle point in terms of the original field component is proportional to $\alpha^{-1}$. Thus, for $\alpha \propto N, N$ the particle number, the variance vanishes proportional to $N^{-1}$ in the thermodynamic limit $N \rightarrow \infty$. For example, the integral in eq. A.2a could be again rewritten as

$$
\mathrm{e}^{-\mathcal{H}[w]}:=\mathrm{e}^{\alpha|w|^{2}}=\frac{\alpha}{\pi} \iint \mathrm{d} \hat{u} \mathrm{~d} \hat{v} \mathrm{e}^{\alpha|w|^{2}-\alpha\left\{(\hat{u}-\operatorname{Re} w)^{2}+(\hat{v}-\operatorname{Im} w)^{2}\right\}},
$$

showing that the variance of $\hat{u}$ with this measure is

$$
\left\langle|\hat{u}-\operatorname{Re} w|^{2}\right\rangle_{\mathcal{H}[w]}=\alpha^{-1} \propto N^{-1},
$$

independently of the realization of the original field $w$ or the system configuration (analogously for $\hat{v}-\operatorname{Im} w$ ). Thus, each integral in eqs. (A.2) is dominated by the contribution of the saddle-point value of a conjugated (interaction) field in terms of the original field. Neither a subsequent configurational average, e.g., with the Gaussian-chain weight, nor the choice of $c$ in eq. A.3 affect this saddle-point relation.

\section{A.1.2 Application to our partition function}

The inner integrals in eq. (3.8) due to the HS transformations have, for any set of polymer configurations, $\mathcal{S}$, the (exact) saddle points

$$
\begin{array}{ll}
\overline{\hat{\sigma}_{k}^{\prime}}=\operatorname{Re} \sigma_{\boldsymbol{k}}^{(\mathcal{S})}, & \overline{\hat{\sigma}_{\boldsymbol{k}}^{\prime \prime}}=\operatorname{Im} \sigma_{\boldsymbol{k}}^{(\mathcal{S})}, \\
\overline{\hat{\varrho}_{\boldsymbol{k}}^{\prime}}=i \operatorname{Re} \varrho_{\boldsymbol{k}}^{(\mathcal{S})}, & \overline{\hat{\varrho}_{\boldsymbol{k}}^{\prime \prime}}=i \operatorname{Im} \varrho_{\boldsymbol{k}}^{(\mathcal{S})}
\end{array}
$$

of the interaction fields in terms of the collective densities. For the components of the interaction field $\hat{\varrho}$ at one $\boldsymbol{k}$, the situation is sketched in fig. A.1. From eqs. (3.5)

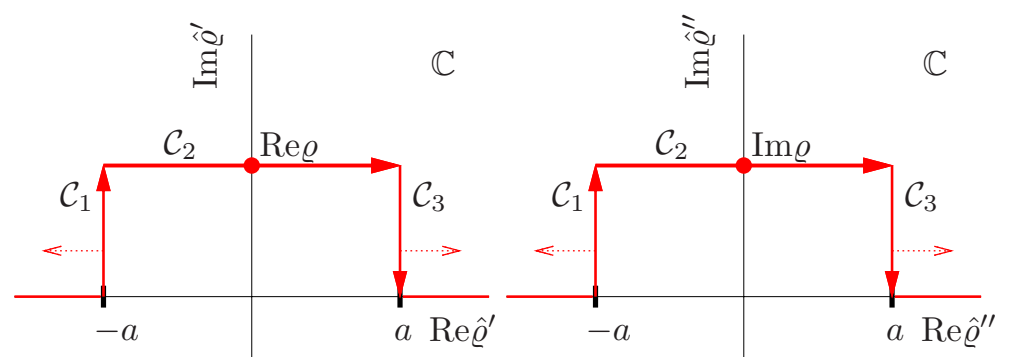

Figure A.1: Saddle-point contours, cf., e.g., [14, for components of the density interaction field. The limit $a \rightarrow \infty$ eliminates the contributions from $\mathcal{C}_{1}$ and $\mathcal{C}_{3}$, and the integral along $\mathcal{C}_{2}$, parallel to the real axis, is dominated by the contribution at the saddle point $(\bullet)$.

and (3.11), we can moreover confirm that ensemble averages of modes of the collective densities and of the conjugated interaction fields with the weight of eq. (3.11), i.e., including the configurational average, are proportional to each other (cf. appendix A.3.1). 


\section{A.2 Quadratic theory for arbitrary sequence distributions}

In the general case of arbitrary Gaussian-chain copolymer sequences or (non-Markovian) sequence distributions without $A \leftrightharpoons B$ exchange symmetry, the expansion of the effective Hamiltonian $\hat{h}$, cf. eq. 3.15, up to second order in $\hat{\sigma}, \hat{\varrho}$ is

$$
\begin{aligned}
\hat{h}[\hat{\sigma}, \hat{\varrho}]= & \sum_{\nu} p_{\nu} \ln p_{\nu} \\
& +\frac{1}{8 \varrho_{0}^{2}} \sum_{\boldsymbol{k} \neq \mathbf{0}}\left(2 L \chi-\chi^{2} \sum_{\nu} p_{\nu} \sum_{s_{1}, s_{2}}\left(q_{\nu}\left(s_{1}\right)-q\right)\left(q_{\nu}\left(s_{2}\right)-q\right) \mathrm{e}^{-\left|s_{2}-s_{1}\right| k^{2}}\right) \hat{\sigma}_{\boldsymbol{k}} \hat{\sigma}_{-\boldsymbol{k}} \\
& -i \frac{\chi \kappa}{4 \varrho_{0}^{2}} \sum_{\boldsymbol{k} \neq \mathbf{0}} \sum_{\nu} p_{\nu} \sum_{s_{1}, s_{2}}\left(q_{\nu}\left(s_{1}\right)-q\right) \mathrm{e}^{-\left|s_{2}-s_{1}\right| k^{2}} \hat{\sigma}_{\boldsymbol{k}} \hat{\varrho}_{-\boldsymbol{k}} \\
& +\frac{\kappa^{2}}{8 \varrho_{0}^{2}} \sum_{\boldsymbol{k} \neq \mathbf{0}}\left(\frac{2 L}{\kappa}+\sum_{s_{1}, s_{2}} \mathrm{e}^{-\left|s_{2}-s_{1}\right| k^{2}}\right) \hat{\varrho}_{\boldsymbol{k}} \hat{\varrho}_{-\boldsymbol{k}}+\mathcal{O}\left(\hat{\varrho}_{\boldsymbol{k}}^{3}, \hat{\varrho}_{\boldsymbol{k}}^{2} \hat{\sigma}_{\boldsymbol{k}}, \hat{\varrho}_{\boldsymbol{k}} \hat{\sigma}_{\boldsymbol{k}}^{2}, \hat{\sigma}_{\boldsymbol{k}}^{3}, \ldots\right) .
\end{aligned}
$$

Elimination of $\hat{\rho}$ with the saddle-point relation analogous to eq. 3.24 shows that the generalization of the second-order structure function $S\left(k^{2}\right)$ in eq. (3.25), decisive for the instability of the disordered, homogeneous state, reads

$$
\tilde{S}\left(k^{2}\right)=\sum_{\nu} p_{\nu} \sum_{s_{1}, s_{2}} q_{\nu}\left(s_{1}\right) q_{\nu}\left(s_{2}\right) \mathrm{e}^{-\left|s_{2}-s_{1}\right| k^{2}}-\frac{\left(\sum_{\nu} p_{\nu} \sum_{s_{1}, s_{2}} q_{\nu}\left(s_{1}\right) \mathrm{e}^{-\left|s_{2}-s_{1}\right| k^{2}}\right)^{2}}{\sum_{\nu} p_{\nu} \sum_{s_{1}, s_{2}} \mathrm{e}^{-\left|s_{2}-s_{1}\right| k^{2}}} .
$$

Here, $\nu$ indexes individual sequences, not sequence classes. The last term vanishes only if each sequence $\nu$ and its complement $\bar{\nu}$, cf. eq. (B.5), have equal concentrations in the melt. Note that the general expression eq. A.9 has to be considered especially for a single (asymmetric) sequence.

\section{A.2.1 Relation to random phase approximation}

The second-order structure function eq. A.9 is proportional to the function $F_{q}, q=k^{2}$, defining the scattering function of the disordered melt within random phase approximation (RPA) [76, 45, see sec. A.2.2 below. This can be seen by rewriting eq. A.9) using the definition of our type variable, $q(s)=\delta_{s, A}-\delta_{s, B}$, where $\delta_{s, J}=1$, if $s$ is of type $J$, and zero otherwise. First, we have

$$
\begin{aligned}
& \sum_{\nu} p_{\nu} \sum_{s_{1}, s_{2}} q_{\nu}\left(s_{1}\right) q_{\nu}\left(s_{2}\right) \mathrm{e}^{-\left|s_{2}-s_{1}\right| k^{2}} \\
& =\sum_{\nu} p_{\nu} \sum_{s_{1}, s_{2}}\left(\delta_{\left(\nu, s_{1}\right), A}-\delta_{\left(\nu, s_{1}\right), B}\right)\left(\delta_{\left(\nu, s_{2}\right), A}-\delta_{\left(\nu, s_{2}\right), B}\right) \mathrm{e}^{-\left|s_{2}-s_{1}\right| k^{2}} \\
& =S_{A A}\left(k^{2}\right)+S_{B B}\left(k^{2}\right)-2 S_{A B}\left(k^{2}\right)
\end{aligned}
$$

cf. the correlation defined in eq. A.20 below. Similarly, using $1=\delta_{s, A}+\delta_{s, B}$, we find

$$
\sum_{\nu} p_{\nu} \sum_{s_{1}, s_{2}} q_{\nu}\left(s_{1}\right) \mathrm{e}^{-\left|s_{2}-s_{1}\right| k^{2}}=S_{A A}\left(k^{2}\right)-S_{B B}\left(k^{2}\right)
$$


and

$$
\sum_{\nu} p_{\nu} \sum_{s_{1}, s_{2}} \mathrm{e}^{-\left|s_{2}-s_{1}\right| k^{2}}=S_{A A}\left(k^{2}\right)+S_{B B}\left(k^{2}\right)+2 S_{A B}\left(k^{2}\right),
$$

which finally gives for $\tilde{S}\left(k^{2}\right)$

$$
\tilde{S}\left(k^{2}\right)=4 \frac{S_{A A}\left(k^{2}\right) S_{B B}\left(k^{2}\right)-\left(S_{A B}\left(k^{2}\right)\right)^{2}}{S_{A A}\left(k^{2}\right)+S_{B B}\left(k^{2}\right)+2 S_{A B}\left(k^{2}\right)},
$$

proportional to the response function eq. A.19.

\section{A.2.2 Response function within RPA}

The RPA approach employs the fluctuation-response theorem to calculate the response function of the copolymer melt, cf., e.g., [117]. Generally in linear response, the Fourier amplitudes $\Phi_{k}^{(A)}$ of an $A$ (excess) density wave due to an excitation $\Psi$ (with amplitudes $\Psi_{k}$ ) that acts on $A$ segments only, are

$$
\Phi_{\boldsymbol{k}}^{(A)}=\alpha_{\boldsymbol{k}}^{\chi} \Psi_{\boldsymbol{k}}
$$

with response coefficients $\alpha_{\boldsymbol{k}}^{\chi}$. First, the aim is to determine the response without incompatibility between segment types $A$ and $B$ (sometimes called athermal case),

$$
\Phi_{k}^{(A)}=\alpha_{k} \Psi_{k}
$$

In order to calculate $\alpha_{\boldsymbol{k}}$ for the incompressible system, one introduces an internal field $\hat{\Psi}$ which acts both on $A$ and on $B$ segments and which imposes conservation of the sum of $A$ and $B$ segment densities:

$$
\begin{aligned}
& \Phi_{\boldsymbol{k}}^{(A)}=\alpha_{\boldsymbol{k}}^{A A}\left(\Psi_{\boldsymbol{k}}+\hat{\Psi}_{\boldsymbol{k}}\right)+\alpha_{\boldsymbol{k}}^{A B} \hat{\Psi}_{\boldsymbol{k}} \\
& \Phi_{\boldsymbol{k}}^{(B)}=-\Phi_{\boldsymbol{k}}^{(A)}=\alpha_{\boldsymbol{k}}^{B B} \hat{\Psi}_{\boldsymbol{k}}+\alpha_{\boldsymbol{k}}^{B A}\left(\hat{\Psi}_{\boldsymbol{k}}+\Psi_{\boldsymbol{k}}\right)
\end{aligned}
$$

(since $A$ and $B$ waves must have equal amplitudes, but opposite signs in the incompressible system). Herein, $\alpha_{\boldsymbol{k}}^{J K}$ is the coefficient for the response of $J$ segments due to an excitation of (chemically coupled) $K$ segments on the same copolymer. Solving for $\hat{\Psi}_{k}$, one finds

$$
\hat{\Psi}_{\boldsymbol{k}}=-\frac{\alpha_{\boldsymbol{k}}^{A A}+\alpha_{\boldsymbol{k}}^{B A}}{\alpha_{\boldsymbol{k}}^{A A}+\alpha_{\boldsymbol{k}}^{A B}+\alpha_{\boldsymbol{k}}^{B A}+\alpha_{\boldsymbol{k}}^{B B}} \Phi_{\boldsymbol{k}}^{(A)}
$$

and thus for the response coefficient without $A B$ repulsion, using $\alpha_{\boldsymbol{k}}^{B A}=\alpha_{\boldsymbol{k}}^{A B}$ for the correlation functions,

$$
\alpha_{\boldsymbol{k}}=\frac{\alpha_{\boldsymbol{k}}^{A A} \alpha_{\boldsymbol{k}}^{B B}-\left(\alpha_{\boldsymbol{k}}^{A B}\right)^{2}}{\alpha_{\boldsymbol{k}}^{A A}+\alpha_{\boldsymbol{k}}^{B B}+2 \alpha_{\boldsymbol{k}}^{A B}} .
$$

The $\alpha_{\boldsymbol{k}}^{J K}$ are Fourier-transformed density-density correlations (due to connectivity only) of type $J$ with type $K$ segments residing on one copolymer, averaged over the sequence distribution, e.g., the correlation of $A$ with $A$ is

$$
\alpha_{\boldsymbol{k}}^{A A}=\sum_{\nu} p_{\nu} \sum_{s_{1}, s_{2}} \delta_{\left(\nu, s_{1}\right), A} \delta_{\left(\nu, s_{2}\right), A} \mathrm{e}^{-\left|s_{2}-s_{1}\right| k^{2}}=: S_{A A}\left(k^{2}\right),
$$


for Gaussian chains with discrete segments, cf. sec. C. The function eq. A.19) is seen to be (up to a prefactor) the second-order structure function eq. A.9), cf. eq. A.13.

On the basis of the $\alpha_{\boldsymbol{k}}$, the response with incompatibility $\chi$ can be calculated with the following mean-field argument. The excitation $\Psi_{\boldsymbol{k}}$ is augmented by $\chi \Phi_{\boldsymbol{k}}^{(A)}$, since $A$ density modulations now act attractively on type $A$ segments,

$$
\Phi_{\boldsymbol{k}}^{(A)}=\alpha_{\boldsymbol{k}}\left(\Psi_{\boldsymbol{k}}+\chi \Phi_{\boldsymbol{k}}^{(A)}\right) \Rightarrow \frac{1}{\alpha_{\boldsymbol{k}}^{\chi}}=\frac{1}{\alpha_{\boldsymbol{k}}}-\chi .
$$

\section{A.3 Relation between second-order vertices of functionals in $\hat{\sigma}$ and in $\sigma$ and relation to scattering function}

Our free-energy functional is not a series expansion in the Fourier amplitudes of the physical $A$ excess density,

$$
\sigma_{\boldsymbol{k}}=\frac{1}{V} \sum_{j=1}^{N} \sum_{s=1}^{L}\left(q_{j}(s)-q\right) \mathrm{e}^{i \boldsymbol{k} \cdot \boldsymbol{r}_{j}(s)},
$$

but in the amplitudes of a conjugated interaction field $\hat{\sigma}$. Ensemble averages of the density $\sigma$ and the interaction field $\hat{\sigma}$ are proportional to each other, their covariances or field fluctuations are not (cf., e.g., [97]), as detailed in sec. A.3.1. Therefore the coefficients in our functional cannot be directly identified with inverse density $(\sigma-)$ correlation functions of the melt. Yet, as long as we only address ensemble averages within mean-field theory we are free to expand the free-energy functional in terms of the interaction field, which then serves as an order parameter. With this choice, the vertices thus do not coincide with those in the free-energy functional in, e.g., ref. [42], but recover the results of the latter theory on the Gaussian level, as will be shown below, in sec. A.3.2.

\section{A.3.1 Averages and covariances of the collective density $\sigma$ and its conjugated field $\hat{\sigma}$}

In order to highlight differences that arise in the coefficients of the free-energy functional, particularly regarding the relation between the second-order coefficient and the inverse scattering function, we consider the relation for the covariances

$$
\left\langle\sigma_{\boldsymbol{k}_{1}} \sigma_{\boldsymbol{k}_{2}}\right\rangle_{\mathrm{cov}}:=\left\langle\sigma_{\boldsymbol{k}_{1}} \sigma_{\boldsymbol{k}_{2}}\right\rangle_{\mathcal{H}}-\left\langle\sigma_{\boldsymbol{k}_{1}}\right\rangle_{\mathcal{H}}\left\langle\sigma_{\boldsymbol{k}_{2}}\right\rangle_{\mathcal{H}} .
$$

For better traceability of this derivation, the Hamiltonian is reduced to incompatibility and connectivity. Introducing an auxiliary field $h$ that couples linearly to $\sigma$, the first two moments of the collective $A$ excess density are obtained as

$$
\begin{aligned}
\left\langle\sigma_{\boldsymbol{k}_{1}}\right\rangle_{\mathcal{H}} & =\left.\frac{\partial \ln \mathcal{Z}_{\sigma}}{\partial h_{-\boldsymbol{k}_{1}}}\right|_{h=0} \\
\left\langle\sigma_{\boldsymbol{k}_{1}} \sigma_{\boldsymbol{k}_{2}}\right\rangle_{\text {cov }} & =\left.\frac{\partial^{2} \ln \mathcal{Z}_{\sigma}}{\partial h_{-\boldsymbol{k}_{1}} \partial h_{-\boldsymbol{k}_{2}}}\right|_{h=0} .
\end{aligned}
$$


from the (generating) partition function

$$
\mathcal{Z}_{\sigma}:=\left\langle\exp \left\{\frac{N L \chi}{4 \varrho_{0}^{2}} \sum_{\boldsymbol{k}} \sigma_{\boldsymbol{k}} \sigma_{-\boldsymbol{k}}+\sigma_{\boldsymbol{k}} h_{-\boldsymbol{k}}\right\}\right\rangle_{\mathcal{H}_{\mathrm{C}}},
$$

where we dropped the combinatorial prefactor $\Omega$.

The HS transform induces additional integrations over the modes of the interaction field $\hat{\sigma}$ (cf. eq. (3.7)),

$\mathcal{Z}_{\sigma}$

$$
\begin{aligned}
& =\left\langle\int \mathcal{D}[\hat{\sigma}] \prod_{\boldsymbol{k}} \exp \left\{-\frac{N L \chi}{4 \rho_{0}^{2}} \hat{\sigma}_{\boldsymbol{k}} \hat{\sigma}_{-\boldsymbol{k}}-\frac{\varrho_{0}^{2}}{N L \chi} h_{\boldsymbol{k}} h_{-\boldsymbol{k}}+\frac{N L \chi}{2 \varrho_{0}^{2}} \hat{\sigma}_{\boldsymbol{k}}\left(\sigma_{-\boldsymbol{k}}+\frac{2 \rho_{0}^{2}}{N L \chi} h_{-\boldsymbol{k}}\right)\right\}\right\rangle_{\mathcal{H}_{\mathrm{C}}} \\
& =\int \mathcal{D}[\hat{\sigma}] \exp \left\{-\frac{\varrho_{0}^{2}}{N L \chi} \sum_{\boldsymbol{k}}\left(h_{\boldsymbol{k}}-\frac{N L \chi}{2 \varrho_{0}^{2}} \hat{\sigma}_{\boldsymbol{k}}\right)\left(h_{-\boldsymbol{k}}-\frac{N L \chi}{2 \varrho_{0}^{2}} \hat{\sigma}_{-\boldsymbol{k}}\right)+\sum_{\nu} N_{\nu} \ln z_{\nu}[\hat{\sigma}]\right\},
\end{aligned}
$$

with

$$
z_{\nu}[\hat{\sigma}]=\left\langle\exp \left\{\frac{\chi}{2 \varrho_{0}} \sum_{\boldsymbol{k}} \hat{\sigma}_{\boldsymbol{k}} \sum_{s}\left(q_{\nu}(s)-q\right) \mathrm{e}^{-i \boldsymbol{k} \cdot \boldsymbol{r}(s)}\right\}\right\rangle
$$

(with $\langle\cdot\rangle$ the single-chain average eq. $(3.13)$ ). Thus, for the averages one has

$$
\left\langle\sigma_{\boldsymbol{k}_{1}}\right\rangle_{\mathcal{H}}=\left.\frac{\partial \ln \mathcal{Z}_{\sigma}}{\partial h_{-\boldsymbol{k}_{1}}}\right|_{h=0}=\left\langle\hat{\sigma}_{\boldsymbol{k}_{1}}\right\rangle_{N \hat{h}},
$$

for the variances, however:

$$
\begin{aligned}
\left\langle\sigma_{\boldsymbol{k}_{1}} \sigma_{\boldsymbol{k}_{2}}\right\rangle_{\text {cov }} & \\
= & -\frac{2 \varrho_{0}^{2}}{N L \chi} \frac{\partial}{\partial h_{-\boldsymbol{k}_{1}}}\left\{\frac{1}{\mathcal{Z}_{\sigma}} \int \mathcal{D}[\hat{\sigma}]\left(h_{\boldsymbol{k}_{2}}-\frac{N L \chi}{2 \varrho_{0}^{2}} \hat{\sigma}_{\boldsymbol{k}_{2}}\right)\right. \\
& \left.\times \exp \left\{-\frac{\varrho_{0}^{2}}{N L \chi} \sum_{\boldsymbol{k}}\left(h_{\boldsymbol{k}}-\frac{N L \chi}{2 \varrho_{0}^{2}} \hat{\sigma}_{\boldsymbol{k}}\right)\left(h_{-\boldsymbol{k}}-\frac{N L \chi}{2 \varrho_{0}^{2}} \hat{\sigma}_{-\boldsymbol{k}}\right)+\sum_{\nu} N_{\nu} \ln z_{\nu}[\hat{\sigma}]\right\}\right\}\left.\right|_{h=0} \\
= & -\left\langle\hat{\sigma}_{\boldsymbol{k}_{1}}\right\rangle_{N \hat{h}}\left\langle\hat{\sigma}_{\boldsymbol{k}_{2}}\right\rangle_{N \hat{h}}+\left\langle\hat{\sigma}_{\boldsymbol{k}_{1}} \hat{\sigma}_{\boldsymbol{k}_{2}}\right\rangle_{N \hat{h}}-\frac{2 \varrho_{0}^{2}}{N L \chi} \delta_{\boldsymbol{k}_{1},-\boldsymbol{k}_{2}} \\
= & \left\langle\hat{\sigma}_{\boldsymbol{k}_{1}} \hat{\sigma}_{\boldsymbol{k}_{2}}\right\rangle_{\mathrm{cov}}-\frac{2 \varrho_{0}^{2}}{N L \chi} \delta_{\boldsymbol{k}_{1},-\boldsymbol{k}_{2}}
\end{aligned}
$$

\section{A.3.2 Comparison with Fredrickson et al.}

The order and set of approximations as well as the order-parameter field in our freeenergy functional are different from those chosen by Fredrickson et al. [42. We expand the effective Hamiltonian in eq. A.26) (at $h=0$ ) in the amplitudes of the interaction field $\hat{\sigma}$ (which, according to the saddle-point relation, must be small if those of the collective density are). The authors of ref. [42] first introduce functional integrations 
over both $\sigma$ and an auxiliary field $\hat{\sigma}$, the latter to fix $\sigma$ to the physical collective density. In our model notation of eq. A.26, this procedure amounts to writing

$$
\mathcal{Z}_{\sigma}=\int \mathcal{D}[\hat{\sigma}, \sigma] \exp \left\{\sum_{\boldsymbol{k}}\left(\frac{N L \chi}{4 \varrho_{0}^{2}} \sigma_{\boldsymbol{k}} \sigma_{-\boldsymbol{k}}+\left(i \hat{\sigma}_{-\boldsymbol{k}}+h_{-\boldsymbol{k}}\right) \sigma_{\boldsymbol{k}}\right)+N \sum_{\nu} p_{\nu} \ln \hat{z}_{\nu}[\hat{\sigma}]\right\}
$$

with the single-sequence partition functions

$$
\hat{z}_{\nu}[\hat{\sigma}]:=\left\langle\exp \left\{-\frac{i}{V} \sum_{\boldsymbol{k}} \hat{\sigma}_{-\boldsymbol{k}} \sum_{s}\left(q_{\nu}(s)-q\right) \mathrm{e}^{i \boldsymbol{k} \cdot \boldsymbol{r}(s)}\right\}\right\rangle
$$

Then, they expand the logarithm of the partition function up to fourth order in the field $\hat{\sigma}$, in order to eliminate $\hat{\sigma}$ at the saddle point in favor of the (averaged) collective density $\sigma$. In doing so, Fredrickson et al. restrict their theory to the limit of many blocks per chain and perform the expansion with certain further approximations, which we do not impose. Observing these restrictions, the vertex functions arising in the expansions of their functional and of (A.27) can be mapped onto each other. Our second-order vertex function in a $\lambda$-distribution and for $q=0$, is for discrete segments given by $S\left(k^{2}\right)$ from eq. C.14, and in the continuous-chain limit by $s\left(k^{2}\right)$, eq. C.15. The function $s(x) / Q$ gives the function $G_{2}(x) /\left(C_{2} M\right)$ (eq. (B.19) in ref. [42]) when invoking the approximations of large $Q$ and $\left|\lambda^{Q}\right| \ll 1$ of ref. [42]. Thus, the relation to our discrete structure function in the symmetric case $\left(f=1 / 2\right.$ or $\left.C_{2}=1 / 4\right)$ is

$$
G_{2}\left(k^{2}\right) \hat{=} \frac{1}{4 L} S\left(k^{2} / M\right) .
$$

With this correspondence, Fredrickson et al. obtain, from their terms quadratic in $\hat{\sigma}$, the saddle point

$$
\overline{\hat{\sigma}_{\boldsymbol{k}}}=\frac{i V^{2}}{4 N L G_{2}\left(k^{2}\right)} \sigma_{\boldsymbol{k}}
$$

and the effective Hamiltonian (per chain) up to second order

$$
\frac{L}{8 \varrho_{0}^{2}} \sum_{\boldsymbol{k}}\left(\frac{1}{G_{2}\left(k^{2}\right)}-2 \chi\right) \sigma_{\boldsymbol{k}} \sigma_{-\boldsymbol{k}} .
$$

The inverse coefficient of this quadratic term then gives the Fourier-transformed $A$ excess density-density correlation or variance within saddle-point approximation,

$$
\frac{4 \varrho_{0}^{2}}{N L}\left(\frac{1}{G_{2}\left(k^{2}\right)}-2 \chi\right)^{-1} \approx\left\langle\sigma_{\boldsymbol{k}} \sigma_{-\boldsymbol{k}}\right\rangle_{\mathrm{cov}}
$$

and is therefore in ref. [42] identified as the scattering function $\Sigma\left(k^{2}\right) \propto\left\langle\sigma_{\boldsymbol{k}} \sigma_{-\boldsymbol{k}}\right\rangle_{\text {cov }}$ measurable in experiments (not to be confused with our function $S\left(k^{2}\right)$ ).

On the other hand, expanding the effective Hamiltonian in eq. A.26) in our interaction field $\hat{\sigma}$ yields the second-order term

$$
\frac{L \chi}{4 \varrho_{0}^{2}} \sum_{\boldsymbol{k}}\left(1-\chi \frac{S\left(k^{2} / M\right)}{2 L}\right) \hat{\sigma}_{\boldsymbol{k}} \hat{\sigma}_{-\boldsymbol{k}} \hat{=} \frac{L \chi}{4 \varrho_{0}^{2}} \sum_{\boldsymbol{k}}\left(1-2 \chi G_{2}\left(k^{2}\right)\right) \hat{\sigma}_{\boldsymbol{k}} \hat{\sigma}_{-\boldsymbol{k}},
$$


cf. eqs. 3.18$)$ or $(3.25)$, the $\hat{=}$ indicating that the left-hand expression reverts to the right-hand expression with the approximations of ref. [42] for the structure function, cf. eq. A.31). Obviously, the instability occurs at the same incompatibility $\chi$ in both cases. If we now insert the variance of our field $\hat{\sigma}$ at the saddle-point level from the coefficient in eq. (A.35), cf. eq. (3.35), into the relation eq. (A.28b), we can recover the (mean-field) variance of the A excess density as

$$
\frac{2 \varrho_{0}^{2}}{N L \chi\left(1-2 \chi G_{2}\left(k^{2}\right)\right)}-\frac{2 \varrho_{0}^{2}}{N L \chi} \approx\left\langle\sigma_{\boldsymbol{k}} \sigma_{-\boldsymbol{k}}\right\rangle_{\mathrm{cov}},
$$

which is the result from eq. A.34). Due to the different order of saddle-point and small-amplitudes approximation, and due to the restriction to many-block chains in ref. [42, we abstain from comparing the fourth-order terms and the lamellar free energy. 


\section{B Composition and sequence distribution of random $Q$-block copolymers}

\section{B.1 Number and classes of $Q$-block sequences}

Here, our goal is to motivate the contributions to $\mathcal{N}(Q)$ (cf. eq. 2.6), the number of possible different sequences or species in a $Q$-block copolymer melt. Due to the symmetry with respect to the two ends of a polymer, $\mathcal{N}(Q)$ is smaller than $2^{Q}$ for $Q \geq 2$. First, we single out the set of sequences with a symmetry of the following kind: We depict a sequence in a folded state, with two branches of equal length emanating from the folding point. The general procedure to generate a sequence in this set is to assign different types to the two opposite blocks on different branches, as in the example

$$
\left\lceil\frac{Q}{2}\right\rceil\left[\begin{array}{c}
\cdots \\
\cdots
\end{array} \text { or generally } q(\beta)=-q(Q+1-\beta), \quad \beta \in\{1, \ldots, Q\},\right.
$$

with $\lceil x\rceil:=\min (n \in \mathbb{N}: n \geq x)$. According to this prescription, starting from the folding point, only the types of the inner blocks on one branch, e.g., of those with numbers from 2 to $\left\lceil\frac{Q}{2}\right\rceil$, can be chosen freely among $A$ and $B$. Since we do not distinguish the two ends of a polymer, the permutation of the end's block-types is equivalent to the simultaneous permutation of the types of all blocks with numbers 2 to $\left\lfloor\frac{Q}{2}\right\rfloor$ and hence does not contribute another factor 2 for the number of different sequences in this set. Hence the number of sequences in this set (for $Q$ even, the $A \leftrightharpoons B$ exchange-symmetric sequences) is

$$
\mathcal{N}_{\text {symm }}(Q)=2^{\left\lceil\frac{Q}{2}\right\rceil-1} .
$$

A sequence from the second, disjoint set, without the above mentioned symmetry, is generated by fixing the type of at least one block $\beta$ as equal to that of the opposite block on the other branch as $q(\beta)=q(Q+1-\beta)$. Therefore, this second set comprises

$$
\mathcal{N}_{\text {asymm }}(Q)=2^{Q-1}
$$

sequences, and the total number of different species is

$$
\mathcal{N}(Q)=\mathcal{N}_{\text {symm }}(Q)+\mathcal{N}_{\text {asymm }}(Q)=2^{\left\lceil\frac{Q}{2}\right\rceil-1}+2^{Q-1} .
$$

In this context, we define the complement $\bar{\nu}$ of a sequence $\nu$ as the sequence obtained from $\nu$ by exchanging $A$ and $B$,

$$
q_{\bar{\nu}}(s)=-q_{\nu}(s) \quad \forall s=1, \ldots, L
$$

(for $Q$ even, $\nu$ and $\bar{\nu}$ can actually be the same sequence, such as $A A B B$ and $B B A A$ from the first, symmetric set). In globally $A \leftrightharpoons B$ exchange-symmetric distributions, $\nu$ 
and $\bar{\nu}$ have equal concentrations and coincide in the structure functions of even order (see, e.g., eq. (C.9) below). For these distributions, it is convenient to combine $\nu$ and $\bar{\nu}$ into one sequence class. An example is the sequence class indexed by 2 for triblock copolymers, $\{A A B, B B A\}$.

\section{B.2 Transition matrix and block-type correlation of Markovian sequences}

In terms of the global probability $p$ to find a block of type $A$ and the correlation $\lambda$ of the types of adjacent blocks (defined in section 2.1), the transition matrix reads

$$
\hat{M}=\left(\begin{array}{cc}
p(1-\lambda)+\lambda & p(1-\lambda) \\
(1-p)(1-\lambda) & 1-p(1-\lambda)
\end{array}\right) .
$$

Note that for ideal random $Q$-block sequences with $\lambda=0$, the composition (or $A$ content) distribution is binomial and thus has variance $p(1-p) / Q$. For the special case $p=1 / 2$ (symmetric random block copolymers) mostly referred to in this work, $\hat{M}$ is

$$
\hat{M}=\frac{1}{2}\left(\begin{array}{cc}
1+\lambda & 1-\lambda \\
1-\lambda & 1+\lambda
\end{array}\right)
$$

thus a block of the same type is attached with probability $(1+\lambda) / 2$. Powers of $\hat{M}$ are needed to compute the type correlation of non-adjacent blocks and can be accessed via the diagonalization

$$
\hat{M}=\underbrace{\left(\begin{array}{cc}
p & 1 \\
1-p & -1
\end{array}\right)}_{\hat{T}} \underbrace{\left(\begin{array}{ll}
1 & 0 \\
0 & \lambda
\end{array}\right)}_{\hat{D}} \underbrace{\left(\begin{array}{cc}
1 & 1 \\
1-p & -p
\end{array}\right)}_{\hat{T}^{-1}} .
$$

Since $M$ segments of the same type form one block, and in order to address single monomer positions for the structure functions in section C, it is convenient to introduce the block number of segment $s$ as

$$
\beta(s)=\left\lceil\frac{s}{M}\right\rceil .
$$

The conditional probabilities to find segment $s_{2}$ of type $J$, separated from segment $s_{1}$ of type $K$ on the same chain by $\Delta \beta:=\left|\beta\left(s_{1}\right)-\beta\left(s_{2}\right)\right|$ blocks, with $J, K \in\{A, B\}$ mapped onto the matrix indices $\{1,2\}$, are, according to $(\mathrm{B} .8)$, the entries of the matrix

$$
\hat{M}^{\Delta \beta}=\left(\begin{array}{cc}
p+(1-p) \lambda^{\Delta \beta} & p\left(1-\lambda^{\Delta \beta}\right) \\
(1-p)\left(1-\lambda^{\Delta \beta}\right) & 1-p+p \lambda^{\Delta \beta}
\end{array}\right) .
$$

\section{B.3 Moments of composition distribution}

Basic characteristics of the block-type sequence distribution are the variance in $A$ content (composition) and higher moments, determined by averaged intra-chain type 
correlations. For a Markovian distribution defined by $p$ and $\lambda$, they can be calculated using the transition matrix $\hat{M}$ presented in appendix B.2. These moments however do not distinguish sequences which have equal $A$ contents, but different internal topologies of $A$ - $B$ contacts.

For a given sequence $\nu$, the $n$th moment of composition is simply the $n$th power of the net type $A$ excess of this sequence:

$$
m_{n, \nu}:=\frac{1}{L^{n}}\left(\sum_{s=1}^{L} q_{\nu}(s)\right)^{n}, n \in \mathbb{N} .
$$

The corresponding $n$th moment of a distribution of sequences, characterized by the sequence concentrations $\left\{p_{\nu}\right\}$, is defined as

$$
\begin{aligned}
m_{n} & :=\frac{1}{L^{n}} \sum_{s_{1}, \ldots, s_{n}}\left[q_{\nu}\left(s_{1}\right) q_{\nu}\left(s_{2}\right) \cdots q_{\nu}\left(s_{n}\right)\right]_{p_{\nu}} \\
& =\frac{1}{L^{n}} \sum_{\nu} p_{\nu} \sum_{s_{1}, \ldots, s_{n}} q_{\nu}\left(s_{1}\right) q_{\nu}\left(s_{2}\right) \cdots q_{\nu}\left(s_{n}\right) .
\end{aligned}
$$

In a $\lambda$-defined distribution, the average $\left[f\left(q\left(s_{1}\right), \ldots, q\left(s_{n}\right)\right)\right]_{\lambda}$ over all sequences can be performed using the conditional probabilities in (B.10) and the average $A$ content $p$, which determines the first moment as $q:=m_{1}=2 p-1$. In order to obtain the second moment, we compute the type correlation of two segments on the same chain whose block numbers differ by $\Delta \beta \in\{0, \ldots, Q-1\}$, corrected for the average $A$ excess $q$ :

$$
\begin{aligned}
& {\left[\left(q\left(s_{1}\right)-q\right)\left(q\left(s_{2}\right)-q\right)\right]_{\lambda}:=\sum_{\nu} p_{\nu}(\lambda)\left(q_{\nu}\left(s_{1}\right)-q\right)\left(q_{\nu}\left(s_{2}\right)-q\right)} \\
& =\hat{M}_{A A}^{\Delta \beta} p-\hat{M}_{B A}^{\Delta \beta} p-\hat{M}_{A B}^{\Delta \beta}(1-p)+\hat{M}_{B B}^{\Delta \beta}(1-p)-q^{2} \\
& =\left(1-q^{2}\right) \lambda^{\Delta \beta}
\end{aligned}
$$

Analogously as in eq. (B.13), we calculate the type correlation of three segments whose two smallest block distances are given by $\Delta \beta_{1}$ and $\Delta \beta_{2}$ :

$$
\begin{aligned}
& {\left[\left(q\left(s_{1}\right)-q\right)\left(q\left(s_{2}\right)-q\right)\left(q\left(s_{3}\right)-q\right)\right]_{\lambda}} \\
& =\left[q\left(s_{1}\right) q\left(s_{2}\right) q\left(s_{3}\right)\right]_{\lambda}-q \sum_{1 \leq i<j \leq 3}\left[\left(q\left(s_{i}\right)-q\right)\left(q\left(s_{j}\right)-q\right)\right]_{\lambda}-q^{3} \\
& =-2 q\left(1-q^{2}\right) \lambda^{\Delta \beta_{1}+\Delta \beta_{2}}=-2 q\left(1-q^{2}\right) \lambda^{\beta\left(s_{3}\right)-\beta\left(s_{1}\right)}
\end{aligned}
$$

In the following, the type variable will always be defined relative to the average $A$ excess,

$$
(q(s)-q) \rightarrow q(s),
$$

and the moments correspondingly as centered moments,

$$
m_{n}:=\frac{1}{L^{n}} \sum_{\nu} p_{\nu} \sum_{s_{1}, \ldots, s_{n}}\left(q_{\nu}\left(s_{1}\right)-q\right) \cdots\left(q_{\nu}\left(s_{n}\right)-q\right),
$$


in order to free the expressions from products of lower moments and powers of $q$. With this definition, four segments have the type correlation

$$
\begin{aligned}
& {\left[q\left(s_{1}\right) q\left(s_{2}\right) q\left(s_{3}\right) q\left(s_{4}\right)\right]_{\lambda}} \\
& =4 q^{2}\left(1-q^{2}\right) \lambda^{\beta\left(s_{4}\right)-\beta\left(s_{1}\right)}+\left(1-q^{2}\right)^{2} \lambda^{\beta\left(s_{4}\right)-\beta\left(s_{3}\right)+\beta\left(s_{2}\right)-\beta\left(s_{1}\right)}
\end{aligned}
$$

In eqs. (B.14) and (B.17), we assumed a prior permutation of the segment indices $\left\{s_{j}\right\}$ such that $i<j \Rightarrow \beta\left(s_{i}\right) \leq \beta\left(s_{j}\right)$, to avoid extensive use of minima and maxima of the block distances.

\section{B.3.1 Second moment}

Summing eq. (B.13) over all segments' block distances $\Delta \beta$ gives the second-order moment in a Markovian, $\lambda$-defined sequence distribution:

$$
\begin{aligned}
m_{2} & :=\frac{1}{L^{2}} \sum_{s_{1}, s_{2}=1}^{L}\left[q\left(s_{1}\right) q\left(s_{2}\right)\right]_{\lambda} \\
& =\frac{1-q^{2}}{L^{2}}\left\{\sum_{s_{1}, s_{2}=1}^{M} Q+2 M^{2} \sum_{\Delta \beta=1}^{Q-1}(Q-\Delta \beta) \lambda^{\Delta \beta}\right\} \\
& =\left(1-q^{2}\right) \frac{M^{2}}{L^{2}}\left\{Q+2\left((Q+1)-\frac{\mathrm{d}}{\mathrm{d} \lambda} \lambda\right) \lambda \sum_{\Delta \beta^{\prime}=0}^{Q-2} \lambda^{\Delta \beta^{\prime}}\right\} \\
& =\frac{1-q^{2}}{Q^{2}} \frac{Q\left(1-\lambda^{2}\right)-2 \lambda\left(1-\lambda^{Q}\right)}{(1-\lambda)^{2}} \\
& =\frac{1-q^{2}}{Q}\left\{1+\frac{2 \lambda\left(Q-1-\sum_{n=1}^{Q-1} \lambda^{n}\right)}{Q(1-\lambda)}\right\} \\
& =\frac{1-q^{2}}{Q}\left\{1+\frac{2 \lambda \sum_{n=0}^{Q-2}(Q-1-n) \lambda^{n}}{Q}\right\}
\end{aligned}
$$

with the representation in the last line of eq. (B.18), particularly suitable for small $Q$. (Note the trivial result $q_{2}=1-q^{2}$ in the case $\lambda=+1$.) For symmetric triblocks $(q=0$ and $Q=3$ ) one finds:

$$
m_{2}=\frac{1}{3}+\frac{2 \lambda(2+\lambda)}{9} .
$$




\section{B.3.2 Fourth moment}

From eq. (B.17), we derive the fourth moment in a symmetric $(q=0), \lambda$-defined distribution:

$$
\begin{aligned}
& m_{4}:=\frac{1}{L^{4}} \sum_{s_{1}, s_{2}, s_{3}, s_{4}=1}^{L}\left[q\left(s_{1}\right) q\left(s_{2}\right) q\left(s_{3}\right) q\left(s_{4}\right)\right]_{\lambda} \\
& =\{Q+6 \underbrace{\sum_{\Delta \beta_{2}=1}^{Q-1}\left(Q-\Delta \beta_{2}\right)}_{Q(Q-1) / 2}+8 \sum_{\Delta \beta_{1}=1}^{Q-1}\left(Q-\Delta \beta_{1}\right) \lambda^{\Delta \beta_{1}} \\
& +12 \sum_{\Delta \beta_{1}=1}^{Q-2} \sum_{\Delta \beta_{3}=1}^{Q-1-\Delta \beta_{1}}\left(Q-\Delta \beta_{1}-\Delta \beta_{3}\right) \lambda^{\Delta \beta_{1}+\Delta \beta_{3}} \\
& +24 \sum_{\Delta \beta_{1}=1}^{Q-2} \sum_{\Delta \beta_{2}=1}^{Q-1-\Delta \beta_{1}}\left(Q-\Delta \beta_{1}-\Delta \beta_{2}\right) \lambda^{\Delta \beta_{1}} \\
& \left.+24 \sum_{\Delta \beta_{1}=1}^{Q-3} \sum_{\Delta \beta_{2}=1}^{Q-2-\Delta \beta_{1}} \sum_{\Delta \beta_{3}=1}^{Q-1-\Delta \beta_{1}-\Delta \beta_{2}}\left(Q-\Delta \beta_{1}-\Delta \beta_{2}-\Delta \beta_{3}\right) \lambda^{\Delta \beta_{1}+\Delta \beta_{3}}\right\} \cdot \frac{1}{Q^{4}} \\
& =\frac{3 Q-2}{Q^{3}}+\underbrace{\frac{4 \lambda(3 Q-4)\left(Q-1-\sum_{n=1}^{Q-1} \lambda^{n}\right)}{Q^{4}(1-\lambda)}}_{L_{2}} \\
& -\underbrace{\frac{12 \lambda^{2}\left\{Q\left(2 \lambda^{Q-1}-(Q-1)(1-\lambda)+4\right)-6 \sum_{n=0}^{Q-1} \lambda^{n}\right\}}{Q^{4}(1-\lambda)^{3}}}_{L_{3}}
\end{aligned}
$$

with the expanded expressions suitable for small $Q$ :

$$
Q^{4} L_{2}=4 \lambda(3 Q-4) \sum_{n=0}^{Q-2}(Q-1-n) \lambda^{n}
$$

and

$$
Q^{4} L_{3}=12 \lambda^{2} \sum_{n=0}^{Q-4}(n+1)(Q-2-n)(Q-3-n) \lambda^{n}
$$

(the last term appears if four pairwise different block numbers are possible and thus is relevant for $Q>3$ only). 



\section{Structure functions}

In this chapter, we compute the structure functions, technically vertex functions of the free-energy functional, for the $A \leftrightharpoons B$ symmetric case, cf. eqs. (3.18), (3.25), and (3.32). For the definitions, we choose the expressions for individual block-type sequences $\nu$; the average over any sequence distribution is a superposition of the form eq. (3.23). As a prerequisite, we derive general features and the number of independent arguments of the structure functions, in sec. C.1. Subsequently, we calculate the structure functions, averaged over a $\lambda$-defined sequence distribution ${ }^{1}$ in sec. C.5. and sequence-specific in sec. C.6.

Wave numbers $k:=|\boldsymbol{k}|$ are normalized with the rms end-to-end distance $b$ of a Kuhn statistical segment [in the continuous-chain limit, with a block's rms end-to-end distance $\sqrt{M} b], k^{2}:=b^{2} \tilde{k}^{2} /(2 d)\left[k^{2}:=M b^{2} \tilde{k}^{2} /(2 d)\right]$, where $\tilde{k}$ is the physical wave number.

\section{C.1 Gaussian-chain conformational averages}

Here, we derive the Fourier modes or characteristic functions of the conformational probability density of a Gaussian chain (with discrete segments), which appear in the vertices of the expansions eqs. 3.18), 3.25), and (3.32). With the normalization of the single-chain measure, anticipating the thermodynamic limit, $V \rightarrow \infty$,

$$
\begin{aligned}
& \lim _{V \rightarrow \infty} \frac{1}{V} \int \mathcal{D}[\boldsymbol{r}(s)] \exp \left\{-\frac{1}{4} \sum_{s=1}^{L}(\boldsymbol{r}(s)-\boldsymbol{r}(s-1))^{2}\right\} \\
= & \lim _{V \rightarrow \infty} \frac{1}{V} \int \mathrm{d}^{d} r(0) \int \mathcal{D}[\boldsymbol{b}(s)] \exp \left\{-\frac{1}{4} \sum_{s=1}^{L} \boldsymbol{b}(s) \cdot \boldsymbol{b}(s)\right\}=(4 \pi)^{L d / 2},
\end{aligned}
$$

and using

$$
\boldsymbol{r}\left(s_{\mathrm{r}}\right)=\boldsymbol{r}(0)+\sum_{s=1}^{L} \boldsymbol{b}(s) \Theta_{s_{\mathrm{r}}-s}
$$

( $\Theta$ the discrete Heaviside function), the $n$-point correlations are

$$
\begin{aligned}
& \left\langle\exp \left\{-i \sum_{\mathrm{r}=1}^{n} \boldsymbol{k}_{\mathrm{r}} \cdot \boldsymbol{r}\left(s_{\mathrm{r}}\right)\right\}\right\rangle \\
= & \lim _{V \rightarrow \infty} \frac{1}{V}(4 \pi)^{-L d / 2} \int \mathrm{d}^{d} r(0) \exp \left\{-i \sum_{\mathrm{r}=1}^{n} \boldsymbol{k}_{\mathrm{r}} \cdot \boldsymbol{r}(0)\right\} \\
& \times \int \mathcal{D}[\boldsymbol{b}(s)] \exp \left\{-\frac{1}{4} \sum_{s=1}^{L} \boldsymbol{b}(s) \cdot \boldsymbol{b}(s)-i \sum_{\mathrm{r}=1}^{n} \boldsymbol{k}_{\mathrm{r}} \cdot \sum_{s=1}^{L} \boldsymbol{b}(s) \Theta_{s_{\mathrm{r}}-s}\right\} .
\end{aligned}
$$

\footnotetext{
${ }^{1}$ Diagrammatic techniques for the calculation of vertex functions in a Landau free energy of $A B$ multiblock copolymers, with a replica approach, may be found in 3, 4. Explicit expressions ensue only for special distributions.
} 
Evaluation of eq. (C.3) with $\min (a, b)=(a+b-|a-b|) / 2$ gives

$$
\begin{aligned}
& \left\langle\exp \left\{-i \sum_{\mathrm{r}=1}^{n} \boldsymbol{k}_{\mathrm{r}} \cdot \boldsymbol{r}\left(s_{\mathrm{r}}\right)\right\}\right\rangle \\
& =\delta_{\sum_{\mathrm{r}} \boldsymbol{k}_{\mathrm{r}}, \mathbf{0}} \exp \left\{-\sum_{s=1}^{L}\left(\sum_{\mathrm{r}=1}^{n} \boldsymbol{k}_{\mathrm{r}} \Theta_{s_{\mathrm{r}}-s}\right)^{2}\right\} \\
& =\delta_{\sum_{\mathrm{r}} \boldsymbol{k}_{\mathrm{r}}, \mathbf{0}} \exp \left\{-\sum_{\mathrm{r}, \mathrm{r}^{\prime}=1}^{n} \boldsymbol{k}_{\mathrm{r}} \cdot \boldsymbol{k}_{\mathrm{r}^{\prime}} \sum_{s=1}^{L} \Theta_{\min \left(s_{\mathrm{r}}, s_{\mathrm{r}^{\prime}}\right)-s}\right\} \\
& =\delta_{\sum_{\mathrm{r}} \boldsymbol{k}_{\mathrm{r}}, \mathbf{0}} \exp \left\{-\frac{1}{2} \sum_{\mathrm{r}, \mathrm{r}^{\prime}=1}^{n}\left(s_{\mathrm{r}}+s_{\mathrm{r}^{\prime}}\right) \boldsymbol{k}_{\mathrm{r}} \cdot \boldsymbol{k}_{\mathrm{r}^{\prime}}+\frac{1}{2} \sum_{\mathrm{r}, \mathrm{r}^{\prime}=1}^{n}\left|s_{\mathrm{r}}-s_{\mathrm{r}^{\prime}}\right| \boldsymbol{k}_{\mathrm{r}} \cdot \boldsymbol{k}_{\mathrm{r}^{\prime}}\right\} \\
& =\delta_{\sum_{\mathrm{r}} \boldsymbol{k}_{\mathrm{r}}, \mathbf{0}} \exp \left\{\sum_{1=\mathrm{r}<\mathrm{r}^{\prime}}^{n}\left|s_{\mathrm{r}^{\prime}}-s_{\mathrm{r}}\right| \boldsymbol{k}_{\mathrm{r}} \cdot \boldsymbol{k}_{\mathrm{r}^{\prime}}\right\} .
\end{aligned}
$$

with $\delta$ the Kronecker symbol in $d$ dimensions. Due to the uniformity of the probability density with respect to the coordinates of a single chain's center of mass, contributions to the characteristic function arise only if the $n$ wave vectors sum to zero. The conformational average (C.4) can be computed similarly for continuous Gaussian chains (see e.g. 47], appendix B: Wiener correlator), with the same result.

In order to sort and calculate the contributions to $n$-point structure functions, eq. (C.4) can be rewritten for ordered segment indices $\left\{s_{j}\right\}$, such that $i<j \Rightarrow s_{i} \leq s_{j}$, as

$$
\begin{aligned}
& \delta_{\sum_{\mathrm{r}} \boldsymbol{k}_{\mathrm{r}}, \mathbf{0}} \exp \left\{\sum_{1=\mathrm{r}<\mathrm{r}^{\prime}}^{n}\left|s_{\mathrm{r}}-s_{\mathrm{r}^{\prime}}\right| \boldsymbol{k}_{\mathrm{r}} \cdot \boldsymbol{k}_{\mathrm{r}^{\prime}}\right\} \\
& =\delta_{\sum_{\mathrm{r}} \boldsymbol{k}_{\mathrm{r}}, \mathbf{0}} \exp \left\{\sum_{\mathrm{r}^{\prime}=1}^{n} s_{\mathrm{r}^{\prime}}\left[\left(\boldsymbol{k}_{\mathrm{r}^{\prime}}+\sum_{\mathrm{r}<\mathrm{r}^{\prime}} \boldsymbol{k}_{\mathrm{r}}\right)^{2}-\left(\sum_{\mathrm{r}<\mathrm{r}^{\prime}} \boldsymbol{k}_{\mathrm{r}}\right)^{2}\right]\right\} \\
& =\exp \left\{-\sum_{\mathrm{r}=1}^{n-1}\left(s_{\mathrm{r}+1}-s_{\mathrm{r}}\right)\left(\sum_{\mathrm{r}^{\prime}=1}^{\mathrm{r}} \boldsymbol{k}_{\mathrm{r}^{\prime}}\right)^{2}\right\} .
\end{aligned}
$$

\section{C.2 Debye function}

\section{C.2.1 Discrete segments}

The second-order structure or density-density correlation function of a homopolymer (subsequence) of length $L$ is, with $\langle\cdot\rangle$ the conformational Gaussian-chain average 
calculated in eq. (C.4), for discrete segments

$$
\begin{aligned}
D\left(L, k^{2}\right): & =\left.\sum_{s_{1}, s_{2}=1}^{L}\left\langle\mathrm{e}^{-i\left(\boldsymbol{k}_{1} \cdot \boldsymbol{r}\left(s_{1}\right)+\boldsymbol{k}_{2} \cdot \boldsymbol{r}\left(s_{2}\right)\right)}\right\rangle\right|_{\boldsymbol{k}:=\boldsymbol{k}_{1}} \\
& =\sum_{s_{1}, s_{2}=1}^{L} \mathrm{e}^{-\left|s_{2}-s_{1}\right| k^{2}} \\
& =L+2 \sum_{s_{1}<s_{2}}^{L} \mathrm{e}^{-\left(s_{2}-s_{1}\right) k^{2}}=L+2 \sum_{\Delta s=1}^{L-1}(L-\Delta s) \mathrm{e}^{-\Delta s k^{2}} \\
& =L \frac{1+\mathrm{e}^{-k^{2}}}{1-\mathrm{e}^{-k^{2}}}-2 \mathrm{e}^{-k^{2}} \frac{1-\mathrm{e}^{-L k^{2}}}{\left(1-\mathrm{e}^{-k^{2}}\right)^{2}} .
\end{aligned}
$$

\section{C.2.2 Continuous-chain limit}

The structure function for a continuous-chain Gaussian homopolymer, comprising $\ell$ blocks of equal length, is

$$
d\left(\ell, k^{2}\right)=2 \int_{0}^{\ell} \mathrm{d} s_{1} \int_{0}^{s_{1}} \mathrm{~d} s_{2} \mathrm{e}^{-\left(s_{1}-s_{2}\right) k^{2}}=\frac{\mathrm{e}^{-\ell k^{2}}-1+\ell k^{2}}{k^{4} / 2}
$$

Here, $k$ is rescaled with the rms end-to-end-distance of a block.

\section{C.3 Second-order structure function}

The second-order structure function $S_{\nu}\left(k^{2}\right)$ for an arbitrary sequence $\nu$ of length $L$ is

$$
\begin{aligned}
S_{\nu}\left(k^{2}\right): & =\left.\sum_{s_{1}, s_{2}=1}^{L} q_{\nu}\left(s_{1}\right) q_{\nu}\left(s_{2}\right)\left\langle\mathrm{e}^{-i\left(\boldsymbol{k}_{1} \cdot \boldsymbol{r}\left(s_{1}\right)+\boldsymbol{k}_{2} \cdot \boldsymbol{r}\left(s_{2}\right)\right)}\right\rangle\right|_{\boldsymbol{k}:=\boldsymbol{k}_{1}} \\
& =\sum_{s_{1}, s_{2}=1}^{L} q_{\nu}\left(s_{1}\right) q_{\nu}\left(s_{2}\right) \mathrm{e}^{-\left|s_{2}-s_{1}\right| k^{2}}
\end{aligned}
$$

and thus, generally, a polynomial in $\exp \left(-k^{2}\right)$, a property which can be exploited at small $Q$ and $L$ to find the maxima with respect to $k$. By construction, $S_{\nu}\left(k^{2}\right)$ is nonnegative for all $k$. In compact form, each $S_{\nu}\left(k^{2}\right)$ can be represented as a linear combination of Debye functions, cf. sec. C.2. The required splitting of the sums can be exemplified for a simple case, the structure function for class $2, A A B / B B A$, of the triblock copolymer sequences, cf. eqs. (3.44):

$$
\begin{aligned}
S_{2}\left(k^{2}\right) & =\left\{\sum_{s_{1}, s_{2}=1}^{2 M}+\sum_{s_{1}, s_{2}=2 M+1}^{3 M}-2 \sum_{s_{1}=1}^{2 M} \sum_{s_{2}=2 M+1}^{3 M}\right\} \mathrm{e}^{-\left|s_{2}-s_{1}\right| k^{2}} \\
& =D\left(2 M, k^{2}\right)+D\left(M, k^{2}\right)-\left(D\left(3 M, k^{2}\right)-D\left(2 M, k^{2}\right)-D\left(M, k^{2}\right)\right) \\
& =-D\left(3 M, k^{2}\right)+2 D\left(2 M, k^{2}\right)+2 D\left(M, k^{2}\right) .
\end{aligned}
$$




\section{C.4 Three-point and fourth-order structure functions}

Structure functions of two and three wave vectors appear in the fourth-order vertices of eq. 3.25). The local fourth-order vertex $\left(\propto \int \mathrm{d}^{d} r \hat{\tau}^{4}(\boldsymbol{r})\right.$ in real space) comprises two terms: The first term in eq. (3.25) due to the density interaction modes features the square of the three-point correlation

$$
S_{\nu}^{(\alpha)}\left(\boldsymbol{k}_{1}, \boldsymbol{k}_{2}\right):=\sum_{s_{1}, s_{2}, s_{3}=1}^{L} q_{\nu}\left(s_{1}\right) q_{\nu}\left(s_{2}\right)\left\langle\mathrm{e}^{-i \sum_{\mathrm{r}=1}^{3} \boldsymbol{k}_{\mathrm{r}} \cdot \boldsymbol{r}\left(s_{\mathrm{r}}\right)}\right\rangle .
$$

The second term contains the structure function

$$
S_{\nu}^{(\beta)}\left(\boldsymbol{k}_{1}, \boldsymbol{k}_{2}, \boldsymbol{k}_{3}\right):=\sum_{s_{1}, s_{2}, s_{3}, s_{4}=1}^{L} q_{\nu}\left(s_{1}\right) q_{\nu}\left(s_{2}\right) q_{\nu}\left(s_{3}\right) q_{\nu}\left(s_{4}\right)\left\langle\mathrm{e}^{-i \sum_{\mathrm{r}=1}^{4} \boldsymbol{k}_{\mathrm{r}} \cdot \boldsymbol{r}\left(s_{\mathrm{r}}\right)}\right\rangle .
$$

The structure function in the nonlocal ('squared gradient square') fourth-order vertex is

$$
\begin{aligned}
& S_{\nu}^{(\gamma)}\left(k_{1}^{2}, k_{2}^{2}\right) \\
& :=\sum_{s_{1}, s_{2}, s_{3}, s_{4}=1}^{L} q_{\nu}\left(s_{1}\right) q_{\nu}\left(s_{2}\right) q_{\nu}\left(s_{3}\right) q_{\nu}\left(s_{4}\right)\left\langle\mathrm{e}^{-i\left(\boldsymbol{k}_{1} \cdot \boldsymbol{r}\left(s_{1}\right)+\boldsymbol{k}_{3} \cdot \boldsymbol{r}\left(s_{3}\right)\right)}\right\rangle\left\langle\mathrm{e}^{-i\left(\boldsymbol{k}_{2} \cdot \boldsymbol{r}\left(s_{2}\right)+\boldsymbol{k}_{4} \cdot \boldsymbol{r}\left(s_{4}\right)\right)}\right\rangle . \\
& =S_{\nu}\left(k_{1}^{2}\right) S_{\nu}\left(k_{2}^{2}\right) .
\end{aligned}
$$

In terms of the second-order structure functions $S_{\nu}\left(k^{2}\right)$ for each sequence $\nu$, cf. eq. (C.8), and the sequence concentrations $p_{\nu}$, the averaged $S^{(\gamma)}\left(k_{1}^{2}, k_{2}^{2}\right)$ can be expressed as

$$
S^{(\gamma)}\left(k_{1}^{2}, k_{2}^{2}\right)=\sum_{\nu} p_{\nu} S_{\nu}\left(k_{1}^{2}\right) S_{\nu}\left(k_{2}^{2}\right)
$$

and therefore is nonnegative for an arbitrary sequence distribution $\left\{p_{\nu}\right\}$ for all $k_{1}, k_{2}$.

\section{C.5 Structure functions in a symmetric Markovian sequence distribution}

\section{C.5.1 Second-order structure function}

Inserting the type correlation of two segments $\Delta \beta$ blocks apart, eq. (B.13), into eq. (3.23) and performing the sum over all pairs, yields the expression eq. 3.37) for the second- 
order structure function $S\left(k^{2}\right)$ in a (Markovian) $\lambda$-distribution:

$$
\begin{aligned}
S\left(k^{2}\right) & =\sum_{s_{1}, s_{2}=1}^{L}\left[q\left(s_{1}\right) q\left(s_{2}\right)\right]_{\lambda} \mathrm{e}^{-\left|s_{2}-s_{1}\right| k^{2}} \\
& =\sum_{s_{1}, s_{2}=1}^{L} \lambda^{\left|\beta\left(s_{2}\right)-\beta\left(s_{1}\right)\right|} \mathrm{e}^{-\left|s_{2}-s_{1}\right| k^{2}} \\
& =Q \sum_{s_{1}, s_{2}=1}^{M} \mathrm{e}^{-\left|s_{2}-s_{1}\right| k^{2}}+2 \sum_{\Delta \beta=1}^{Q-1}(Q-\Delta \beta) \lambda^{\Delta \beta} \sum_{s_{1}=1}^{M} \sum_{s_{2}=\Delta \beta M+1}^{(\Delta \beta+1) M} \mathrm{e}^{-\left|s_{2}-s_{1}\right| k^{2}} \\
& =Q D\left(M, k^{2}\right)+\left(D\left(Q, M k^{2}-\overline{\ln }(\lambda)\right)-Q\right) \sum_{s_{1}, s_{2}=1}^{M} \mathrm{e}^{-\left(s_{2}-s_{1}\right) k^{2}} \\
& =Q D\left(M, k^{2}\right)+2 \lambda \frac{\mathrm{e}^{-M k^{2}}}{1-\lambda \mathrm{e}^{-M k^{2}}}\left(Q-\frac{1-\left(\lambda \mathrm{e}^{-M k^{2}}\right)^{Q}}{1-\lambda \mathrm{e}^{-M k^{2}}}\right) \frac{\sinh ^{2}\left(\frac{M k^{2}}{2}\right)}{\sinh ^{2}\left(\frac{k^{2}}{2}\right)}
\end{aligned}
$$

(this expression had been given earlier in [125]). The argument $M k^{2}-\overline{\ln }(\lambda)$ of the Debye function is symbolic notation: powers of $\mathrm{e}^{-k^{2}}$ in eq. $\mathrm{C} .6$ must be replaced by the respective powers of $\lambda \mathrm{e}^{-M k^{2}}$.

\section{Continuous-chain limit}

For a Markovian distribution of continuous-chain $Q$-block copolymers one obtains

$$
\begin{aligned}
s\left(k^{2}\right): & =\lim _{M \rightarrow \infty} \frac{S\left(k^{2} / M\right)}{M^{2}} \\
& =Q d\left(1, k^{2}\right)+8 \lambda \frac{\sinh ^{2}\left(\frac{k^{2}}{2}\right) \mathrm{e}^{-k^{2}}}{k^{4}\left(1-\lambda \mathrm{e}^{-k^{2}}\right)}\left(Q-\frac{1-\left(\lambda \mathrm{e}^{-k^{2}}\right)^{Q}}{1-\lambda \mathrm{e}^{-k^{2}}}\right) .
\end{aligned}
$$

Here, $k$ is rescaled with the block size, as in eq. (C.7).

\section{C.5.2 Three-point and fourth-order structure functions}

Here, we sketch our approach in computing the structure functions in the fourth-order vertices, eqs. C.10 to (C.12), of a $\lambda$-distribution for general $Q$, which requires extended sorting of the multiple sums and applying combinatorics. As an example, we give the derivation of $S^{(\alpha)}$ in a $\lambda$-distribution; $S^{(\beta)}, S^{(\gamma)}$, and the continuous-chain versions are constructed similarly. 


\section{Three-point structure function}

The three-point structure function in the local fourth-order vertex is

$$
\begin{aligned}
& S^{(\alpha)}\left(\boldsymbol{k}_{1}, \boldsymbol{k}_{2}\right) \\
& :=\sum_{s_{1}, s_{2}, s_{3}=1}^{L}\left[q\left(s_{1}\right) q\left(s_{2}\right)\right]_{p_{\nu}}\left\langle\mathrm{e}^{-i \boldsymbol{k}_{1} \cdot\left(\boldsymbol{r}\left(s_{1}\right)-\boldsymbol{r}\left(s_{3}\right)\right)-i \boldsymbol{k}_{2} \cdot\left(\boldsymbol{r}\left(s_{2}\right)-\boldsymbol{r}\left(s_{3}\right)\right)}\right\rangle \\
& =\sum_{s_{1}, s_{2}, s_{3}=1}^{L}\left[q\left(s_{1}\right) q\left(s_{2}\right)\right]_{p_{\nu}} \mathrm{e}^{\left|s_{2}-s_{1}\right| \boldsymbol{k}_{1} \cdot \boldsymbol{k}_{2}-\left|s_{3}-s_{2}\right|\left(\boldsymbol{k}_{1} \cdot \boldsymbol{k}_{2}+k_{2}^{2}\right)-\left|s_{3}-s_{1}\right|\left(\boldsymbol{k}_{1} \cdot \boldsymbol{k}_{2}+k_{1}^{2}\right)}
\end{aligned}
$$

For a Markovian sequence distribution, it can be expressed in terms of the block-type correlation parameter $\lambda$ :

$$
\begin{aligned}
& S^{(\alpha)}\left(\boldsymbol{k}_{1}, \boldsymbol{k}_{2}\right)=S^{(\alpha)}\left(\boldsymbol{k}_{2}, \boldsymbol{k}_{1}\right) \\
& =\sum_{s_{1}, s_{2}, s_{3}=1}^{L} \lambda^{\left|\beta\left(s_{2}\right)-\beta\left(s_{1}\right)\right|} \mathrm{e}^{\left|s_{2}-s_{1}\right| \boldsymbol{k}_{1} \cdot \boldsymbol{k}_{2}-\left|s_{3}-s_{2}\right|\left(\boldsymbol{k}_{1} \cdot \boldsymbol{k}_{2}+k_{2}^{2}\right)-\left|s_{3}-s_{1}\right|\left(\boldsymbol{k}_{1} \cdot \boldsymbol{k}_{2}+k_{1}^{2}\right)}
\end{aligned}
$$

Splitting the sums according to the possible combinations of block distances between the $s_{j}$ and observing the symmetries results in the following summands of eq. (C.17):

$$
\begin{aligned}
& \sum_{s_{1}, s_{2}, s_{3}=1}^{M} \mathrm{e}^{\left|s_{2}-s_{1}\right| \boldsymbol{k}_{1} \cdot \boldsymbol{k}_{2}-\left|s_{3}-s_{2}\right|\left(\boldsymbol{k}_{1} \cdot \boldsymbol{k}_{2}+k_{2}^{2}\right)-\left|s_{3}-s_{1}\right|\left(\boldsymbol{k}_{1} \cdot \boldsymbol{k}_{2}+k_{1}^{2}\right)}, \\
& 2 \sum_{\Delta \beta=1}^{Q-1}(Q-\Delta \beta) \\
& \times \sum_{s_{1}, s_{2}=1}^{M} \sum_{s_{3}=\Delta \beta M+1}^{(\Delta \beta+1) M} \mathrm{e}^{\left|s_{2}-s_{1}\right| \boldsymbol{k}_{1} \cdot \boldsymbol{k}_{2}-\left(s_{3}-s_{2}\right)\left(\boldsymbol{k}_{1} \cdot \boldsymbol{k}_{2}+k_{2}^{2}\right)-\left(s_{3}-s_{1}\right)\left(\boldsymbol{k}_{1} \cdot \boldsymbol{k}_{2}+k_{1}^{2}\right)}, \\
& \sum_{\Delta \beta=1}^{Q-1}(Q-\Delta \beta) \lambda^{\Delta \beta} \\
& \times\left\{\sum_{s_{1}=1}^{M} \sum_{s_{2}, s_{3}=\Delta \beta M+1}^{(\Delta \beta+1) M} \mathrm{e}^{\left(s_{2}-s_{1}\right) \boldsymbol{k}_{1} \cdot \boldsymbol{k}_{2}-\left|s_{3}-s_{2}\right|\left(\boldsymbol{k}_{1} \cdot \boldsymbol{k}_{2}+k_{2}^{2}\right)-\left(s_{3}-s_{1}\right)\left(\boldsymbol{k}_{1} \cdot \boldsymbol{k}_{2}+k_{1}^{2}\right)}\right. \\
+ & \left.\sum_{s_{2}=1}^{M} \sum_{(\Delta \beta+1) M}^{\left(s_{1}, s_{3}=\Delta \beta M+1\right.} \mathrm{e}^{\left(s_{1}-s_{2}\right) \boldsymbol{k}_{1} \cdot \boldsymbol{k}_{2}-\left(s_{3}-s_{2}\right)\left(\boldsymbol{k}_{1} \cdot \boldsymbol{k}_{2}+k_{2}^{2}\right)-\left|s_{3}-s_{1}\right|\left(\boldsymbol{k}_{1} \cdot \boldsymbol{k}_{2}+k_{1}^{2}\right)}\right\},
\end{aligned}
$$


and

$$
\begin{aligned}
& 2 \sum_{\Delta \beta=1}^{Q-2} \sum_{\Delta \gamma=1}^{Q-\Delta \beta-1}(Q-\Delta \beta-\Delta \gamma) \\
& \times\left\{\lambda^{\Delta \beta} \sum_{s_{1}=1}^{M} \sum_{s_{2}=\Delta \beta M+1}^{(\Delta \beta+1) M} \sum_{s_{3}=(\Delta \beta+\Delta \gamma) M+1}^{(\Delta \beta+\Delta \gamma+1) M} \mathrm{e}^{-\left(s_{3}-s_{2}\right)\left(\boldsymbol{k}_{1}+\boldsymbol{k}_{2}\right)^{2}-\left(s_{2}-s_{1}\right) k_{1}^{2}}\right. \\
&+\lambda^{\Delta \beta+\Delta \gamma} \sum_{s_{1}=1}^{M} \sum_{s_{2}=(\Delta \beta+\Delta \gamma) M+1}^{(\Delta \beta+\Delta \gamma+1) M} \sum_{s_{3}=\Delta \beta M+1}^{(\Delta \beta+1) M} \mathrm{e}^{-\left(s_{2}-s_{3}\right) k_{2}^{2}-\left(s_{3}-s_{1}\right) k_{1}^{2}} \\
&\left.+\lambda^{\Delta \gamma} \sum_{s_{1}=\Delta \beta M+1}^{\Delta \beta+1) M} \sum_{s_{2}=(\Delta \beta+\Delta \gamma+\Delta) M}^{M} \sum^{M} \mathrm{e}^{-\left(s_{1}-s_{3}\right)\left(\boldsymbol{k}_{1}+\boldsymbol{k}_{2}\right)^{2}-\left(s_{2}-s_{1}\right) k_{2}^{2}}\right\} .
\end{aligned}
$$

The first term, eq. C.17a), is $Q$ times the function

$$
\begin{aligned}
\tilde{G}\left(M, k_{1}^{2}, k_{2}^{2}, k_{+}^{2}\right) \\
:=\sum_{s_{1}, s_{2}, s_{3}=1}^{M} \mathrm{e}^{\left|s_{2}-s_{1}\right| \boldsymbol{k}_{1} \cdot \boldsymbol{k}_{2}-\left|s_{3}-s_{2}\right|\left(\boldsymbol{k}_{1} \cdot \boldsymbol{k}_{2}+k_{2}^{2}\right)-\left|s_{3}-s_{1}\right|\left(\boldsymbol{k}_{1} \cdot \boldsymbol{k}_{2}+k_{1}^{2}\right)} \\
=M+2\left\{\sum_{s_{1}=s_{2}<s_{3}}^{M} \mathrm{e}^{-\left(s_{3}-s_{2}\right) k_{+}^{2}}+\sum_{s_{3}=s_{2}<s_{1}}^{M} \mathrm{e}^{-\left(s_{1}-s_{2}\right) k_{1}^{2}}+\sum_{s_{3}=s_{1}<s_{2}}^{M} \mathrm{e}^{-\left(s_{2}-s_{1}\right) k_{2}^{2}}\right. \\
+\sum_{s_{1}<s_{2}<s_{3}}^{M} \mathrm{e}^{-\left(s_{2}-s_{1}\right) k_{1}^{2}-\left(s_{3}-s_{2}\right) k_{+}^{2}}+\sum_{s_{1}<s_{3}<s_{2}}^{M} \mathrm{e}^{-\left(s_{3}-s_{1}\right) k_{1}^{2}-\left(s_{2}-s_{3}\right) k_{2}^{2}} \\
\left.+\sum_{s_{3}<s_{1}<s_{2}}^{M} \mathrm{e}^{-\left(s_{1}-s_{3}\right) k_{+}^{2}-\left(s_{2}-s_{1}\right) k_{2}^{2}}\right\}
\end{aligned}
$$

with $\boldsymbol{k}_{+}:=\boldsymbol{k}_{1}+\boldsymbol{k}_{2}$, which can be expressed as

$$
\begin{aligned}
& D\left(M, k_{+}^{2}\right)+D\left(M, k_{1}^{2}\right)+D\left(M, k_{2}^{2}\right)-2 M \\
& +2\left\{G\left(M, k_{1}^{2}, k_{2}^{2}\right)+G\left(M, k_{1}^{2}, k_{+}^{2}\right)+G\left(M, k_{+}^{2}, k_{2}^{2}\right)\right\},
\end{aligned}
$$


with $D$ the Debye function, eq. (C.6), and the function

$$
\begin{aligned}
& G\left(L, k_{1}^{2}, k_{2}^{2}\right) \\
:= & \sum_{s_{1}<s_{2}<s_{3}}^{L} \mathrm{e}^{-\left(s_{2}-s_{1}\right) k_{1}^{2}-\left(s_{3}-s_{2}\right) k_{2}^{2}}=G\left(L, k_{2}^{2}, k_{1}^{2}\right) \\
= & \sum_{\Delta s=1}^{L-2} \sum_{\Delta t=1}^{L-1-\Delta s}(L-\Delta s-\Delta t) \mathrm{e}^{-\Delta s k_{1}^{2}-\Delta t k_{2}^{2}} \\
= & \frac{\mathrm{e}^{-\left(k_{1}^{2}+k_{2}^{2}\right)}}{\left(1-\mathrm{e}^{-k_{1}^{2}}\right)\left(1-\mathrm{e}^{-k_{2}^{2}}\right)} \\
& \times\left\{\mathrm{e}^{-k_{1}^{2}}-\mathrm{e}^{-k_{2}^{2}}\right. \\
& \left.\quad+\frac{\mathrm{e}^{-k_{1}^{2}-L k_{2}^{2}}\left(\mathrm{e}^{k_{1}^{2}}+\mathrm{e}^{-k_{1}^{2}}-2\right)-\mathrm{e}^{-k_{2}^{2}-L k_{1}^{2}}\left(\mathrm{e}^{k_{2}^{2}}+\mathrm{e}^{-k_{2}^{2}}-2\right)}{\left(1-\mathrm{e}^{-k_{2}^{2}}-\mathrm{e}^{-k_{1}^{2}}\right)\left(1-\mathrm{e}^{-k_{1}^{2}}\right)\left(1-\mathrm{e}^{-k_{2}^{2}}\right)}\right\} .
\end{aligned}
$$

The second term, eq. C.17b), can be written as

$$
\begin{aligned}
& 2 \sum_{\Delta \sigma=1}^{Q-1}(Q-\Delta \sigma) \sum_{s_{1}, s_{2}=1}^{M} \sum_{s_{3}=\Delta \sigma M+1}^{(\Delta \sigma+1) M} \mathrm{e}^{\left|s_{2}-s_{1}\right| \boldsymbol{k}_{1} \cdot \boldsymbol{k}_{2}-\left(s_{3}-s_{2}\right)\left(\boldsymbol{k}_{2} \cdot \boldsymbol{k}_{+}\right)-\left(s_{3}-s_{1}\right)\left(\boldsymbol{k}_{1} \cdot \boldsymbol{k}_{+}\right)} \\
= & 2 \sum_{\Delta \sigma=1}^{Q-1}(Q-\Delta \sigma) \mathrm{e}^{-\Delta \sigma M k_{+}^{2}} \\
& \times \sum_{s_{3}=1}^{M}\left\{\sum_{s_{1}=1}^{M} \mathrm{e}^{-\left(s_{3}-s_{1}\right) k_{+}^{2}}+\sum_{s_{1}<s_{2}}^{M} \mathrm{e}^{-\left(s_{3}-s_{2}\right) k_{+}^{2}-\left(s_{2}-s_{1}\right) k_{1}^{2}}+\sum_{s_{2}<s_{1}}^{M} \mathrm{e}^{-\left(s_{3}-s_{1}\right) k_{+}^{2}-\left(s_{1}-s_{2}\right) k_{2}^{2}}\right\} \\
= & \left(D\left(Q, M k_{+}^{2}\right)-Q\right) \\
& \times\left\{\frac{\sinh ^{2}\left(\frac{M k_{+}^{2}}{2}\right)}{\sinh ^{2}\left(\frac{k_{+}^{2}}{2}\right)} \frac{\mathrm{e}^{-k_{+}^{2}}-\mathrm{e}^{-(M+1) k_{+}^{2}}}{1-\mathrm{e}^{-k_{+}^{2}}}\left\{F\left(M, k_{1}^{2}, k_{+}^{2}-k_{1}^{2}\right)+F\left(M, k_{2}^{2}, k_{+}^{2}-k_{2}^{2}\right)\right\}\right\},
\end{aligned}
$$

with the function

$$
\begin{aligned}
F\left(L, k_{1}^{2}, k_{2}^{2}\right) & :=\sum_{s_{1}<s_{2}}^{L} \mathrm{e}^{s_{1} k_{1}^{2}+s_{2} k_{2}^{2}} \\
& =\frac{\mathrm{e}^{k_{2}^{2}}}{1-\mathrm{e}^{k_{2}^{2}}}\left\{\frac{\mathrm{e}^{k_{1}^{2}+k_{2}^{2}}-\mathrm{e}^{L\left(k_{1}^{2}+k_{2}^{2}\right)}}{1-\mathrm{e}^{k_{1}^{2}+k_{2}^{2}}}-\frac{\mathrm{e}^{L k_{2}^{2}}\left(\mathrm{e}^{k_{1}^{2}}-\mathrm{e}^{L k_{1}^{2}}\right)}{1-\mathrm{e}^{k_{1}^{2}}}\right\} .
\end{aligned}
$$


The third summand, eq. C.17c , can be written as

$$
\begin{aligned}
& \left(D\left(Q, M k_{1}^{2}-\overline{\ln }(\lambda)\right)-Q\right) \\
& \times\left\{\frac{\sinh ^{2}\left(\frac{M k_{1}^{2}}{2}\right)}{\sinh ^{2}\left(\frac{k_{1}^{2}}{2}\right)} \frac{\mathrm{e}^{k_{1}^{2}}-\mathrm{e}^{(M+1) k_{1}^{2}}}{1-\mathrm{e}^{k_{1}^{2}}}\left\{F\left(M, k_{+}^{2}-k_{1}^{2},-k_{+}^{2}\right)+F\left(M, k_{2}^{2}-k_{1}^{2},-k_{2}^{2}\right)\right\}\right\} \\
& +\left(D\left(Q, M k_{2}^{2}-\overline{\ln }(\lambda)\right)-Q\right) \\
& \times\left\{\frac{\sinh ^{2}\left(\frac{M k_{2}^{2}}{2}\right)}{\sinh ^{2}\left(\frac{k_{2}^{2}}{2}\right)} \frac{\mathrm{e}^{k_{2}^{2}}-\mathrm{e}^{(M+1) k_{2}^{2}}}{1-\mathrm{e}^{k_{2}^{2}}}\left\{F\left(M, k_{+}^{2}-k_{2}^{2},-k_{+}^{2}\right)+F\left(M, k_{1}^{2}-k_{2}^{2},-k_{1}^{2}\right)\right\}\right\},
\end{aligned}
$$

and the three summands of eq. C.17d as two times the expression

$$
\begin{aligned}
& G\left(Q, M k_{1}^{2}-\overline{\ln }(\lambda), M k_{+}^{2}\right) \frac{\sinh \left(\frac{M k_{1}^{2}}{2}\right)}{\sinh \left(\frac{k_{1}^{2}}{2}\right)} \frac{\sinh \left(\frac{M k_{+}^{2}}{2}\right)}{\sinh \left(\frac{k_{+}^{2}}{2}\right)} \frac{\sinh \left(\frac{M\left(k_{+}^{2}-k_{1}^{2}\right)}{2}\right)}{\sinh \left(\frac{k_{+}^{2}-k_{1}^{2}}{2}\right)} \\
& +G\left(Q, M k_{+}^{2}, M k_{2}^{2}-\overline{\ln }(\lambda)\right) \frac{\sinh \left(\frac{M k_{+}^{2}}{2}\right)}{\sinh \left(\frac{k_{+}^{2}}{2}\right)} \frac{\sinh \left(\frac{M k_{2}^{2}}{2}\right)}{\sinh \left(\frac{k_{2}^{2}}{2}\right)} \frac{\sinh \left(\frac{M\left(k_{+}^{2}-k_{2}^{2}\right)}{2}\right)}{\sinh \left(\frac{k_{+}^{2}-k_{2}^{2}}{2}\right)} \\
& +G\left(Q, M k_{1}^{2}-\overline{\ln }(\lambda), M k_{2}^{2}-\overline{\ln }(\lambda)\right) \frac{\sinh \left(\frac{M k_{1}^{2}}{2}\right)}{\sinh \left(\frac{k_{1}^{2}}{2}\right)} \frac{\sinh \left(\frac{M k_{2}^{2}}{2}\right)}{\sinh \left(\frac{k_{2}^{2}}{2}\right)} \frac{\sinh \left(\frac{M\left(k_{1}^{2}-k_{2}^{2}\right)}{2}\right)}{\sinh \left(\frac{k_{1}^{2}-k_{2}^{2}}{2}\right)}
\end{aligned}
$$

Restricting the morphology to a lamellar phase with a single wave vector $\boldsymbol{k}$, we need two rather simple combinations of arguments in eq. (C.17): The first is

$$
S^{(\alpha)}(\boldsymbol{k},-\boldsymbol{k})=\sum_{s_{3}=1}^{L} \sum_{s_{1}, s_{2}=1}^{L}\left[q\left(s_{1}\right) q\left(s_{2}\right)\right]_{p_{\nu}} \mathrm{e}^{-\left|s_{2}-s_{1}\right| k^{2}}=L S\left(k^{2}\right)
$$


and the second is $S^{(\alpha)}(\boldsymbol{k}, \boldsymbol{k})$, which for a $\lambda$-distribution becomes

$$
\begin{aligned}
& S^{(\alpha)}(\boldsymbol{k}, \boldsymbol{k})=S^{(\alpha)}(-\boldsymbol{k},-\boldsymbol{k}) \\
= & Q\left\{2 D\left(M, k^{2}\right)+D\left(M,(2 k)^{2}\right)-2 M+2\left\{2 G\left(M, k^{2},(2 k)^{2}\right)+G\left(M, k^{2}, k^{2}\right)\right\}\right\} \\
& +\left(D\left(Q, M(2 k)^{2}\right)-Q\right) \\
& \times\left\{\frac{\sinh ^{2}\left(\frac{M(2 k)^{2}}{2}\right)}{\sinh ^{2}\left(\frac{(2 k)^{2}}{2}\right)}+2 \frac{\mathrm{e}^{-(2 k)^{2}}-\mathrm{e}^{-(M+1)(2 k)^{2}}}{1-\mathrm{e}^{-(2 k)^{2}}} F\left(M, k^{2},(\sqrt{3} k)^{2}\right)\right\} \\
& +2\left(D\left(Q, M k^{2}-\overline{\ln }(\lambda)\right)-Q\right) \\
& \times\left\{\frac{\sinh ^{2}\left(\frac{M k^{2}}{2}\right)}{\sinh ^{2}\left(\frac{k^{2}}{2}\right)}+\frac{\mathrm{e}^{k^{2}}-\mathrm{e}^{(M+1) k^{2}}}{1-\mathrm{e}^{k^{2}}}\left\{F\left(M,(\sqrt{3} k)^{2},-(2 k)^{2}\right)+F\left(M, 0,-k^{2}\right)\right\}\right\} \\
& +4 G\left(Q, M(2 k)^{2}, M k^{2}-\overline{\ln }(\lambda)\right) \frac{\sinh \left(\frac{M k^{2}}{2}\right)}{\sinh \left(\frac{k^{2}}{2}\right)} \frac{\sinh \left(\frac{M(2 k)^{2}}{2}\right)}{\sinh \left(\frac{(2 k)^{2}}{2}\right)} \frac{\sinh \left(\frac{M(\sqrt{3} k)^{2}}{2}\right)}{\sinh \left(\frac{(\sqrt{3} k)^{2}}{2}\right)} .
\end{aligned}
$$

\section{Local four-point structure function}

Again with reordered segment indices $s_{i}, i=1,2,3,4$, such that the block numbers $\beta\left(s_{i}\right)$ fulfill $\beta\left(s_{i}\right)<\beta\left(s_{j}\right)$ if $i<j$, and $\boldsymbol{k}_{+}:=\boldsymbol{k}_{1}+\boldsymbol{k}_{2}+\boldsymbol{k}_{3}$, the local four-point structure function $S^{(\beta)}$ in a $\lambda$-distribution reads:

$$
\begin{aligned}
& S^{(\beta)}\left(\boldsymbol{k}_{1}, \boldsymbol{k}_{2}, \boldsymbol{k}_{3}\right) \\
= & \sum_{\substack{s_{1}, s_{2}, s_{3}, s_{4}=1\\
}}^{L} \lambda^{\beta\left(s_{4}\right)-\beta\left(s_{3}\right)} \lambda^{\beta\left(s_{2}\right)-\beta\left(s_{1}\right)} \\
& \times \mathrm{e}^{\left|s_{2}-s_{1}\right| \boldsymbol{k}_{1} \cdot \boldsymbol{k}_{2}+\left|s_{3}-s_{1}\right| \boldsymbol{k}_{1} \cdot \boldsymbol{k}_{3}+\left|s_{3}-s_{2}\right| \boldsymbol{k}_{2} \cdot \boldsymbol{k}_{3}-\left|s_{4}-s_{1}\right| \boldsymbol{k}_{1} \cdot \boldsymbol{k}_{+}-\left|s_{4}-s_{2}\right| \boldsymbol{k}_{2} \cdot \boldsymbol{k}_{+}-\left|s_{4}-s_{3}\right| \boldsymbol{k}_{3} \cdot \boldsymbol{k}_{+}} .
\end{aligned}
$$

The computation can be performed analogously as that for $S^{(\alpha)}$ in a $\lambda$-distribution, but we do not present it here, since our fractionation theory requires the functions for individual sequences anyway.

\section{C.6 Sequence-specific three-point and fourth-order structure functions used in the fractionation scheme}

In the case of a few different sequences only, the structure functions can be calculated for each sequence, in order to allow in the free-energy optimization for subsystems with sequence distributions different from the global, $\lambda$-defined one (fractionation). The second-order structure functions of individual triblock sequences, $Q=3$, had been given in sec. 3.3.3. Here, we give explicit expressions for the fourth-order vertices $s^{(\alpha)}(\boldsymbol{k}, \boldsymbol{k})$, $s^{(\beta)}(\boldsymbol{k}, \boldsymbol{k},-\boldsymbol{k})$, and $s^{(\gamma)}\left(k^{2}, k^{2}\right)$ needed in the specific case of a lamellar phase with wave vector $\boldsymbol{k}= \pm k \boldsymbol{n}$ in continuous-chain block copolymers. 


\section{C.6.1 $s_{\nu}^{(\alpha)}$ for individual sequences}

For triblock copolymers, the three-point structure functions for each of the sequence classes defined in eq. 3.42$), s_{\nu}^{(\alpha)}(\boldsymbol{k}, \boldsymbol{k})$, read:

$$
\begin{aligned}
s_{1}^{(\alpha)}(\boldsymbol{k}, \boldsymbol{k})= & \tilde{g}\left(3, k^{2}\right) \\
s_{2}^{(\alpha)}(\boldsymbol{k}, \boldsymbol{k})= & -\tilde{g}\left(3, k^{2}\right)+2\left(\tilde{g}\left(2, k^{2}\right)+\tilde{g}\left(1, k^{2}\right)+\Delta \tilde{g}\left(2,3, k^{2}\right)+\Delta \tilde{g}\left(1,3, k^{2}\right)\right) \\
s_{3}^{(\alpha)}(\boldsymbol{k}, \boldsymbol{k})= & \tilde{g}\left(3, k^{2}\right)-4\left(\tilde{g}\left(2, k^{2}\right)-2 \tilde{g}\left(1, k^{2}\right)\right) \\
& -4\left(\Delta \tilde{g}\left(2,3, k^{2}\right)-\Delta \tilde{g}\left(1,3, k^{2}\right)-2 \Delta \tilde{g}\left(1,2, k^{2}\right)\right)
\end{aligned}
$$

with

$$
\begin{aligned}
\tilde{g}\left(\ell, k^{2}\right): & =6 \iiint_{0}^{\ell} \mathrm{d} s_{1} \mathrm{~d} s_{2} \mathrm{~d} s_{3} \mathrm{e}^{\left|s_{2}-s_{1}\right| k^{2}-2\left|s_{3}-s_{2}\right| k^{2}-2\left|s_{3}-s_{1}\right| k^{2}} \\
& =\frac{1}{12 k^{6}}\left(-\mathrm{e}^{-4 \ell k^{2}}+64 \mathrm{e}^{-\ell k^{2}}+24 \ell k^{2} \mathrm{e}^{-\ell k^{2}}-63+36 \ell k^{2}\right)
\end{aligned}
$$

and

$$
\begin{aligned}
& \Delta \tilde{g}\left(\ell_{1}, \ell_{2}, k^{2}\right) \\
& :=\iint_{0}^{\ell_{1}} \mathrm{~d} s_{1} \mathrm{~d} s_{2} \int_{\ell_{1}}^{\ell_{2}} \mathrm{~d} s_{3} \mathrm{e}^{\left|s_{2}-s_{1}\right| k^{2}-2\left|s_{3}-s_{2}\right| k^{2}-2\left|s_{3}-s_{1}\right| k^{2}} \\
& =\frac{1}{24 k^{6}}\left(\mathrm{e}^{-4 \ell_{1} k^{2}}-\mathrm{e}^{-4 \ell_{2} k^{2}}+4 \mathrm{e}^{-\left(4 \ell_{2}-3 \ell_{1}\right) k^{2}}-3 \mathrm{e}^{-4\left(\ell_{2}-\ell_{1}\right) k^{2}}-4 \mathrm{e}^{-\ell_{1} k^{2}}+3\right) .
\end{aligned}
$$

\section{Diblocks}

For the symmetric $A B$ diblock sequence, the function is

$$
s_{A B}^{(\alpha)}(\boldsymbol{k}, \boldsymbol{k})=-\tilde{g}\left(2, k^{2}\right)+4\left(\tilde{g}\left(1, k^{2}\right)+\Delta \tilde{g}\left(1,2, k^{2}\right)\right),
$$

for homopolymers with two blocks accordingly $s_{A A}^{(\alpha)}(\boldsymbol{k}, \boldsymbol{k})=s_{B B}^{(\alpha)}(\boldsymbol{k}, \boldsymbol{k})=\tilde{g}\left(2, k^{2}\right)$.

\section{C.6.2 $s_{\nu}^{(\beta)}$ for individual sequences}

$$
\begin{aligned}
s_{1}^{(\beta)}(\boldsymbol{k}, \boldsymbol{k},-\boldsymbol{k})= & \tilde{c}\left(3, k^{2}\right) \\
s_{2}^{(\beta)}(\boldsymbol{k}, \boldsymbol{k},-\boldsymbol{k})= & -\tilde{c}\left(3, k^{2}\right)+2\left(\tilde{c}\left(2, k^{2}\right)+\tilde{c}\left(1, k^{2}\right)+\Delta \tilde{c}\left(2,3, k^{2}\right)\right), \\
s_{3}^{(\beta)}(\boldsymbol{k}, \boldsymbol{k},-\boldsymbol{k})= & \tilde{c}\left(3, k^{2}\right)-4\left(\tilde{c}\left(2, k^{2}\right)-2 \tilde{c}\left(1, k^{2}\right)\right) \\
& +4\left(\Delta \tilde{c}\left(1,2, k^{2}\right)-2 \Delta_{2} \tilde{c}\left(1,2,3, k^{2}\right)\right),
\end{aligned}
$$


with

$$
\begin{aligned}
& \tilde{c}\left(\ell, k^{2}\right) \\
& :=8 \int_{0}^{\ell} \mathrm{d} s_{1} \int_{s_{1}}^{\ell} \mathrm{d} s_{2} \int_{s_{2}}^{\ell} \mathrm{d} s_{3} \int_{s_{3}}^{\ell} \mathrm{d} s_{4} \mathrm{e}^{\left\{\left(s_{2}-s_{1}\right)+\left(s_{4}-s_{3}\right)-\left(s_{3}-s_{2}\right)-\left(s_{3}-s_{1}\right)-\left(s_{4}-s_{1}\right)-\left(s_{4}-s_{2}\right)\right\} k^{2}} \\
& +8 \int_{0}^{\ell} \mathrm{d} s_{1} \int_{s_{1}}^{\ell} \mathrm{d} s_{3} \int_{s_{3}}^{\ell} \mathrm{d} s_{2} \int_{s_{2}}^{\ell} \mathrm{d} s_{4} \mathrm{e}^{\left\{\left(s_{2}-s_{1}\right)+\left(s_{4}-s_{3}\right)-\left(s_{2}-s_{3}\right)-\left(s_{1}-s_{3}\right)-\left(s_{4}-s_{1}\right)-\left(s_{4}-s_{2}\right)\right\} k^{2}} \\
& +8 \int_{0}^{\ell} \mathrm{d} s_{1} \int_{s_{1}}^{\ell} \mathrm{d} s_{3} \int_{s_{3}}^{\ell} \mathrm{d} s_{2} \int_{s_{2}}^{\ell} \mathrm{d} s_{4} \mathrm{e}^{\{(s 2-s 1)+(s 4-s 3)-(s 2-s 3)-(s 3-s 1)-(s 4-s 1)-(s 4-s 2)\} k^{2}} \\
& =\frac{1}{18 k^{8}}\left(\mathrm{e}^{-4 \ell k^{2}}-784 \mathrm{e}^{-\ell k^{2}}-240 \ell k^{2} \mathrm{e}^{-\ell k^{2}}+783-540 \ell k^{2}+144 \ell k^{4}\right) \text {, } \\
& \Delta \tilde{c}\left(\ell_{1}, \ell_{2}, k^{2}\right) \\
& :=8 \int_{0}^{\ell_{1}} \mathrm{~d} s_{1} \int_{s_{1}}^{\ell_{1}} \mathrm{~d} s_{3} \int_{\ell_{1}}^{\ell_{2}} \mathrm{~d} s_{2} \int_{s_{2}}^{\ell_{2}} \mathrm{~d} s_{4} \mathrm{e}^{\left\{\left(s_{2}-s_{1}\right)+\left(s_{4}-s_{3}\right)-2\left(s_{3}+s_{4}-s_{2}-s_{1}\right)\right\} k^{2}} \\
& +16 \int_{0}^{\ell_{1}} \mathrm{~d} s_{1} \int_{s_{1}}^{\ell_{1}} \mathrm{~d} s_{3} \int_{\ell_{1}}^{\ell_{2}} \mathrm{~d} s_{2} \int_{s_{2}}^{\ell_{2}} \mathrm{~d} s_{4} \mathrm{e}^{\left\{-\left(s_{3}-s_{1}\right)+\left(s_{4}-s_{2}\right)\right\} k^{2}},
\end{aligned}
$$

and

$$
\begin{aligned}
\Delta_{2} \tilde{c}\left(\ell_{1}, \ell_{2}, \ell_{3}, k^{2}\right):= & 2 \int_{0}^{\ell_{1}} \mathrm{~d} s_{1} \int_{s_{1}}^{\ell_{1}} \mathrm{~d} s_{2} \int_{\ell_{1}}^{\ell_{2}} \mathrm{~d} s_{3} \int_{\ell_{2}}^{\ell_{3}} \mathrm{~d} s_{4} \mathrm{e}^{\left(3 s_{2}+s_{1}-3 s_{3}-s_{4}\right) k^{2}} \\
& +8 \int_{0}^{\ell_{1}} \mathrm{~d} s_{1} \int_{s_{1}}^{\ell_{1}} \mathrm{~d} s_{3} \int_{\ell_{1}}^{\ell_{2}} \mathrm{~d} s_{2} \int_{\ell_{2}}^{\ell_{3}} \mathrm{~d} s_{4} \mathrm{e}^{\left(-s_{3}+s_{1}-s_{4}+s_{2}\right) k^{2},}
\end{aligned}
$$

where we omit the lengthy results of the integrations for $\Delta \tilde{c}$ and $\Delta_{2} \tilde{c}$ (these types of integrals can be evaluated with the aid of maple).

\section{Diblocks}

Again, for the symmetric $A B$ diblock sequence, one finds

$$
s_{A B}^{(\beta)}(\boldsymbol{k}, \boldsymbol{k},-\boldsymbol{k})=-\tilde{c}\left(2, k^{2}\right)+4 \tilde{c}\left(1, k^{2}\right)+2 \Delta \tilde{c}\left(1,2, k^{2}\right),
$$

and $s_{A A}^{(\beta)}(\boldsymbol{k}, \boldsymbol{k},-\boldsymbol{k})=s_{B B}^{(\beta)}(\boldsymbol{k}, \boldsymbol{k},-\boldsymbol{k})=\tilde{c}\left(2, k^{2}\right)$ for homopolymers (two identical blocks).

\section{C.6.3 $s_{\nu}^{(\gamma)}$ for individual sequences}

According to eq. C.12), the functions $s_{\nu}^{(\gamma)}\left(k^{2}, k^{2}\right)$ are obtained as the squares of the second-order functions $s_{\nu}\left(k^{2}\right)$, which for triblocks can be found in sec. 3.3.3.

\section{Diblocks}

For the symmetric $A B$ diblock sequence, one has

$$
s_{A B}^{(\gamma)}\left(k^{2}, k^{2}\right)=\left\{-d\left(2, k^{2}\right)+4 d\left(1, k^{2}\right)\right\}^{2} .
$$




\section{Three-phase equilibrium conditions for random triblock copolymers}

\section{D.1 Derivatives}

From eq. (3.50), the simplified version of the lamellar free energy, and eqs. (3.44), one reads off the following derivatives of $f_{\mathrm{m}}$ with respect to the independent concentrations $n_{2}:=n_{2}^{(\mathrm{m})}, n_{3}:=n_{3}^{(\mathrm{m})}$ :

$$
\begin{aligned}
& \frac{\partial f_{\mathrm{m}}}{\partial n_{\nu}} \\
= & \left\{\frac{2 \chi\left(S_{\nu}\left(k_{\mathrm{m}}^{2}\right)-D\left(3 M, k_{\mathrm{m}}^{2}\right)\right)}{\chi S\left(k_{\mathrm{m}}^{2}\right)-2 L}+\frac{16}{81} \frac{9\left(1-8\left(n_{2}+n_{3}\right) / 9\right)+5}{\left(1-8\left(n_{2}+n_{3}\right) / 9\right)^{2}+1-80\left(n_{2}+n_{3}\right) / 81}\right\} \\
& \times \frac{\left(S\left(k_{\mathrm{m}}^{2}\right)-2 L / \chi\right)^{2} / L^{4}}{\left(1-8\left(n_{2}+n_{3}\right) / 9\right)^{2}+1-80\left(n_{2}+n_{3}\right) / 81}+\ln \frac{n_{\nu}}{1-n_{2}-n_{3}}, \nu=2,3,
\end{aligned}
$$

where $S\left(k_{\mathrm{m}}^{2}\right)$ and $k_{\mathrm{m}}$ are functions of $n_{2}, n_{3}$ via

$$
S\left(k^{2}\right)=D\left(3 M, k^{2}\right)+n_{2}\left(S_{2}\left(k^{2}\right)-D\left(3 M, k^{2}\right)\right)+n_{3}\left(S_{3}\left(k^{2}\right)-D\left(3 M, k^{2}\right)\right)
$$

and

$$
k_{\mathrm{m}}:=\underset{k>0}{\operatorname{argmax}} S\left(k^{2}\right),
$$

such that the derivative of the maximum of $S\left(k^{2}\right), S\left(k_{\mathrm{m}}^{2}\right)$, with respect to $n_{2}$ is simply $S_{2}\left(k_{\mathrm{m}}^{2}\right)$, analogously for $n_{3}$, since $\left.\left(\partial S\left(k^{2}\right) / \partial k\right)\right|_{k=k_{\mathrm{m}}}=0$.

The derivatives of $f_{\mathrm{h}}$ from eq. 3.74 with respect to the concentrations $n_{2}^{(\mathrm{h})}, n_{3}^{(\mathrm{h})}$ are

$$
\frac{\partial f_{\mathrm{h}}}{\partial n_{\nu}^{(\mathrm{h})}}=\ln \left\{4\left(\cosh \frac{L \chi \hat{\tau}_{\mathrm{h}}}{6 \varrho_{0}}\right)^{2}-3\right\}+\ln \frac{n_{\nu}^{(\mathrm{h})}}{1-n_{2}^{(\mathrm{h})}-n_{3}^{(\mathrm{h})}}, \nu=2,3,
$$

with the amplitude $\hat{\tau}_{\mathrm{h}} / \varrho_{0}$ of the homogeneous phases from eq. 4.13.

\section{2 Constraints}

At a given point $(\lambda, \chi)$, the independent parameters $\left(v, n_{2}, n_{3}\right)$ of a lamellar subsystem in random triblocks do not only have to solve eqs. 4.5), but also have to take physically reasonable values, further restricted by the global ( $\lambda$-defined) distribution and by the 
demand that lamellae can exist at all in the subsystem. This means that the parameters have to be within the domain of definition

$$
\begin{aligned}
\mathcal{V}:=\{ & \left(v \in[0,1], n_{2} \in\left[0, \min \left(1, \frac{p_{2}(\lambda)}{v^{(2)}}\right)\right],\right. \\
& \left.n_{3} \in\left[0, \min \left(1-n_{2}, \frac{p_{3}(\lambda)}{v^{(2)}}\right)\right]\right): \\
& \left.k_{\mathrm{m}}\left(n_{2}, n_{3}\right)>0 \text { and } L \chi_{0}\left(n_{2}, n_{3}\right) \leq L \chi\right\} .
\end{aligned}
$$

With the enhanced form eq. (3.54) of the lamellar free energy, the wave number $k_{\mathrm{opt}}\left(n_{2}, n_{3} \mid \chi\right)$ has to be tested instead of $k_{\mathrm{m}}\left(n_{2}, n_{3}\right)$. 


\section{E Macroscopic phase separation in the “crushed polymer" approach}

\section{E.1 Two and three homogenous phases in random diblocks}

In a random symmetric diblock melt with $A B$-content $p_{A B}=(1-\lambda) / 2<2 / 3$, two homogenous phases emerge from the disordered state at

$$
L \chi_{0}=\frac{2}{1-p_{A B}}=\frac{4}{1+\lambda},
$$

with the amplitude $\hat{\tau}_{\mathrm{h}, 2}$ for $L \chi>L \chi_{0}$ determined by

$$
\frac{\hat{\tau}_{\mathrm{h}, 2}}{\varrho_{0}}=\left(1-p_{A B}\right) \tanh \left\{\frac{L \chi \hat{\tau}_{\mathrm{h}, 2}}{2 \varrho_{0}}\right\} .
$$

Within the "crushed polymer" approach, a coexistence of three homogeneous phases, one $A$-rich, one $B$-rich, and one balanced in $A$ and $B$, arises at higher incompatibilities. The equations for the amplitude $\hat{\tau}_{\mathrm{h}, 3}$ and for the joint volume fraction $v$ of the $A$-rich and the $B$-rich phase in this coexistence are

$$
\begin{aligned}
\frac{\hat{\tau}_{\mathrm{h}, 3}}{\varrho_{0}}= & \frac{\left(1-p_{A B}\right) \sinh \left\{\frac{L \chi \hat{\tau}_{\mathrm{h}, 3}}{2 \varrho_{0}}\right\}}{v \cosh \left\{\frac{L \chi \hat{\tau}_{\mathrm{h}, 3}}{2 \varrho_{0}}\right\}+(1-v) \exp \left\{\frac{L \chi \hat{\tau}_{\mathrm{h}, 3}^{2}}{4 \varrho_{0}^{2}}\right\}}, \\
1= & \frac{\left(1-p_{A B}\right) \cosh \left\{\frac{L \chi \hat{\tau}_{\mathrm{h}, 3}}{2 \varrho_{0}}\right\}}{v \cosh \left\{\frac{L \chi \hat{\tau}_{\mathrm{h}, 3}}{2 \varrho_{0}}\right\}+(1-v) \exp \left\{\frac{L \chi \hat{\tau}_{\mathrm{h}, 3}^{2}}{4 \varrho_{0}^{2}}\right\}}+\frac{p_{A B}}{v+(1-v) \exp \left\{\frac{L \chi \hat{\tau}_{\mathrm{h}, 3}^{2}}{4 \varrho_{0}^{2}}\right\}} .
\end{aligned}
$$

The corresponding free-energy density of three homogeneous phases is

$$
\begin{aligned}
f_{\mathrm{h}, 3}= & v \frac{L \chi \hat{\tau}_{\mathrm{h}, 3}^{2}}{4 \varrho_{0}^{2}}-\left(1-p_{A B}\right) \ln \left\{v \cosh \left\{\frac{L \chi \hat{\tau}_{\mathrm{h}, 3}}{2 \varrho_{0}}\right\}+(1-v) \exp \left\{\frac{L \chi \hat{\tau}_{\mathrm{h}, 3}^{2}}{4 \varrho_{0}^{2}}\right\}\right\} \quad \text { E.4 } \\
& -p_{A B} \ln \left\{v+(1-v) \exp \left\{\frac{L \chi \hat{\tau}_{\mathrm{h}, 3}^{2}}{4 \varrho_{0}^{2}}\right\}\right\}+\left(1-p_{A B}\right) \ln \left(1-p_{A B}\right)+p_{A B} \ln p_{A B}
\end{aligned}
$$

The transition incompatibility from two to three homogenous phases can be obtained iteratively only, by computing within the three-phase coexistence the volume fraction $(1-v)$ of the phase balanced in $A$ and $B$ and decreasing $L \chi$ until $(1-v)$ reaches zero. 



\section{List of frequently used symbols}

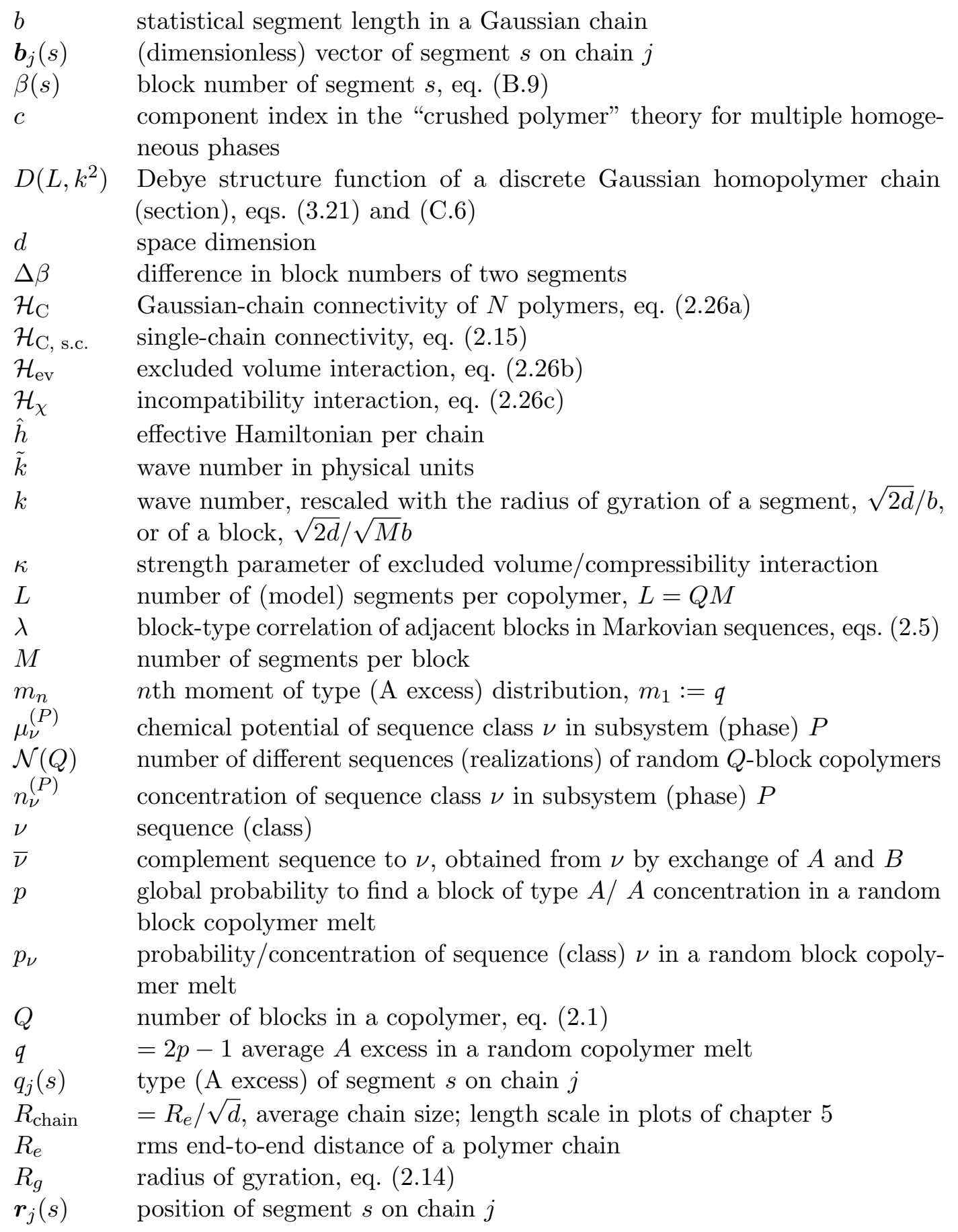


$\varrho_{0} \quad$ dimensionless average segment density

$\varrho \quad$ total segment density field, eq. 2.28

$\varrho \quad$ interaction field conjugated to $\varrho$, the total segment density

$S_{\nu}\left(k^{2}\right) \quad$ second-order structure function for sequence (class) $\nu$, eq. 3.20

$S\left(k^{2}\right) \quad$ global second-order structure function, eq. 3.23 , in a $\lambda$-distribution eq. (C.14)

$\sigma \quad A$ excess density field, eq. 2.30

$\hat{\sigma} \quad$ interaction field conjugated to $\sigma$, the $A$ excess density

$\hat{\tau} \quad$ saddle-point (mean-field) value of $\hat{\sigma}$, field conjugated to $\sigma$

$\chi \quad$ strength parameter of incompatibility between $A$ and $\mathrm{B} ; \chi \propto T^{-1}$

$\chi^{(\mathrm{h})} \quad$ homogeneous cloud transition line to three-phase coexistence

$\chi^{(\mathrm{m})} \quad$ lamellar cloud transition line to three-phase coexistence

$\psi \quad$ critical exponent of order-parameter amplitude

$v \quad$ volume fraction of minority subsystem in a fractionated coexistence

$v^{(m)} \quad$ volume fraction of lamellar subsystem

$\bar{z} \quad$ coordination number on a lattice or average number of nearest neighbors

$\hat{z}_{\nu} \quad$ single-sequence partition function, eq. 3.12

$\mathcal{Z} \quad$ canonical partition function

$\hat{\omega} \quad$ saddle-point value of $\hat{\varrho}$, field conjugated to $\varrho$

$\Omega \quad$ combinatorial (indistinguishability) prefactor in the partition function, eq. $(3.2)$

$[\cdot]_{p_{\nu}} \quad$ average over the block-type sequence distribution

$\langle\cdot\rangle \quad$ conformational average for a single Gaussian chain

$\langle\cdot\rangle_{\mathcal{H}_{\mathrm{C}}} \quad$ conformational average for $N$ Gaussian chains

$\lceil x\rceil \quad:=\min (n \in \mathbb{N}: n \geq x)$ 


\section{Bibliography}

[1] A. Agapov and A. P. Sokolov. Size of the dynamic bead in polymers. Macromolecules 43, 9126 (2010).

[2] S. L. Aggarwal. Structure and properties of block copolymers and multiphase polymer systems: an overview of present status and future potential. Polymer 17(11), 938 (1976).

[3] M. A. Aliev and S. I. Kuchanov. Diagram technique for finding of vertex functions in the Landau theory of heteropolymer liquids. Eur. Phys. J. B 43, 251 (2005).

[4] M. A. Aliev and S. I. Kuchanov. Vertex functions in the Landau theory of phase transitions in melts of Markovian multiblock copolymers. Physica A 387, 363 (2008).

[5] K. Almdal, J. H. Rosedale, F. S. Bates, G. D. Wignall, and G. H. FREDRICKSON. Gaussian- to stretched-coil transition in block copolymer melts. Phys. Rev. Lett. 65(9), 1112 (1990).

[6] H. J. Angermann. The phase behaviour of polydisperse multiblock copolymer melts (a theoretical study). Ph.D. thesis, Rijksuniversiteit Groningen (1998).

[7] H. J. Angermann, G. ten Brinke, and I. Y. Erukhimovich. Microphase separation in correlated random copolymers. Macromolecules 29(9), 3255 (1996).

[8] N. P. BALsara. Thermodynamics of Polymer Blends. In: Physical Properties of Polymers Handbook, edited by J. E. MARK, chap. 19. AIP Press (1996).

[9] J.-L. Barrat and J.-P. Hansen. Basic Concepts for Simple and Complex Liquids. Cambridge University Press (2003).

[10] P. Bartlett and P. B. Warren. Reentrant melting in polydispersed hard spheres. Phys. Rev. Lett. 82, 1979 (1999).

[11] F. S. Bates and G. H. Fredrickson. Block copolymer thermodynamics: Theory and experiment. Annu. Rev. Phys. Chem. 41, 525 (1990).

[12] F. S. BAtes and G. H. Fredrickson. Block copolymers - designer soft materials. Phys. Today 52(2), 32 (1999).

[13] B. J. BAUER. Equilibrium phase compositions of heterogeneous copolymers. Polym. Eng. \& Sci. 25(17), 1081 (1985). 
[14] C. M. Bender and S. A. Orszag. Advanced Mathematical Methods for Scientists and Engineers: Asymptotic Methods and Perturbation Theory. Springer, New York, 2nd edn. (1999).

[15] J. P. Bouchaud and M. E. CAtes. Remarks on the dynamics of random copolymers. J. Phys. II 3, 1171 (1993).

[16] S. A. BrazovskiI. Phase transition of an isotropic system to a nonuniform state. Zh. Eksp. Teor. Fiz. [Sov. Phys. JETP] 68 [41], 175 [85] (1975).

[17] S. A. BrazovskiĬ, I. E. Dzyaloshinskil̆, and A. R. Muratov. Theory of weak crystallization. Sov. Phys. JETP 66(3), 625 (1987).

[18] D. Broseta and G. H. Fredrickson. Phase equilibria in copolymer/homopolymer ternary blends: Molecular weight effects. J. Chem. Phys. 93(4), 2927 (1990).

[19] R. Brout. Statistical Mechanical Theory of a Random Ferrromagnetic System. Phys. Rev. 115(4), 824 (1959).

[20] J. W. CAHn. Phase separation by spinodal decomposition in isotropic systems. J. Chem. Phys. 42(1), 93 (1965).

[21] J. W. CAHn and J. E. Hilliard. Free energy of a nonuniform system. I. Interfacial free energy. J. Chem. Phys. 28(2), 258 (1958).

[22] P. M. Chaikin and T. C. Lubensky. Principles of condensed matter physics. Cambridge University Press, Cambridge (1995).

[23] Y. Cohen, R. J. Albalak, B. J. Dair, M. S. Capel, and E. L. Thomas. Deformation of Oriented Lamellar Block Copolymer Films. Macromolecules 33, $6502(2000)$.

[24] P. DeBye. Molecular-weight determination by light scattering. J. Phys. Colloid. Chem. 51, 18 (1947).

[25] A. V. Dobrynin. Phase coexistence in random copolymers. J. Chem. Phys. 107, 9234 (1997).

[26] A. V. Dobrynin and I. Y. Erukhimovich. Fluctuation theory of weak crystallization in disordered heteropolymer systems. Sov. Phys. JETP Lett. 53, 570 (1991).

[27] A. V. Dobrynin and I. Y. ERukhimovich. Fluctuation theory of random copolymers. J. Phys. I 5, 365 (1995).

[28] M. Doi and S. F. Edwards. The Theory of Polymer Dynamics. Clarendon Press, Oxford (1986). 
[29] E. W. Edwards, M. Müller, M. P. Stoykovich, H. H. Solak, J. J. DE PABlo, and P. F. Nealey. Dimensions and shapes of block copolymer domains assembled on lithographically defined chemically patterned substrates. Macromolecules 40, 90 (2007).

[30] S. F. EdWARDS. The statistical mechanics of polymers with excluded volume. Proc. Phys. Soc. 85, 613 (1965).

[31] S. F. Edwards. The size of a polymer molecule in a strong solution. J. Phys. A: Math. Gen. 8(10), 1670 (1975).

[32] I. Y. ERukhimovich and A. V. Dobrynin. On the theory of weak crystallization in polydisperse molten copolymers. Macromol. Symp. 81, 253 (1994).

[33] M. FAsOlo and P. Sollich. Fractionation effects in phase equilibria of polydisperse hard-sphere colloids. Phys. Rev. E 70, 041410 (2004).

[34] P. J. Flory. Thermodynamics of High Polymer Solutions. J. Chem. Phys. 9, 660 (1941).

[35] P. J. Flory. Thermodynamics of High Polymer Solutions. J. Chem. Phys. 10, 51 (1942).

[36] P. J. FloRY. Thermodynamics of heterogeneous polymers and their solutions. J. Chem. Phys. 12(11), 425 (1944).

[37] P. J. Flory. Principles of Polymer Chemistry. Cornell University Press, Ithaca, NY (1953).

[38] P. J. Flory. Statistical Mechanics of Chain Molecules. InterScience, New York (1969).

[39] G. H. Fredrickson. The Equilibrium Theory of Inhomogeneous Polymers. Clarendon Press, Oxford (2005).

[40] G. H. Fredrickson and E. Helfand. Fluctuation effects in the theory of microphase separation in block copolymers. J. Chem. Phys. 87(1), 697 (1987).

[41] G. H. Fredrickson and S. T. Milner. Thermodynamics of random copolymer melts. Phys. Rev. Lett. 67(7), 835 (1991).

[42] G. H. Fredrickson, S. T. Milner, and L. Leibler. Multicritical phenomena and microphase ordering in random block copolymer melts. Macromolecules $\mathbf{2 5}$, 6341 (1992).

[43] P. G. DE Gennes. Theory of X-ray scattering by liquid macromolecules with heavy atom labels. J. Physique 31, 235 (1970).

[44] P. G. DE Gennes. Scaling Concepts in Polymer Physics. Cornell University Press, Ithaca, NY (1979). 
[45] P. G. De Gennes. Theory of Long-range Correlations in Polymer Melts. Faraday Disc. Chem. Soc. 68, 96 (1979).

[46] J. W. GibBs. Elementary Principles in Statistical Mechanics. Scribners, New York (1902).

[47] P. M. Goldbart, H. Castillo, and A. Zippelius. Randomly crosslinked macromolecular systems: Vulcanization transition to and properties of the amorphous solid state. Adv. Phys. 45(5), 393 (1996).

[48] N. Goldenfeld. Lectures on Phase Transitions and the Renormalization Group. Addison Wesley, Reading (1992).

[49] A. Y. Grosberg and A. R. Khokhlov. Statistical Physics of Macromolecules. AIP Series in Polymers and Complex Materials, New York (1994).

[50] A. M. Gutin, C. D. Sfatos, and E. I. Shakhnovich. Fluctuation effects on microphase separation in random copolymers. J. Phys. A 27, 7957 (1994).

[51] G. Hadzitoannou, C. Picot, A. Skoulios, M.-L. Ionescu, A. Mathis, R. Duplessix, Y. Gallot, and J.-P. Lingelser. Low-angle neutron scattering study of the lateral extension of chains in lamellar styrene/isoprene block copolymers. Macromolecules 15, 263 (1982).

[52] G. Hadzionnnou and A. Skoulios. Structural study of mixtures of styrene/isoprene two- and three-block copolymers. Macromolecules 15, 267 (1982).

[53] D. A. Hajduk, P. E. Harper, S. M. Gruner, C. C. Honeker, G. Kim, and E. L. Thomas. The Gyroid: A New Equilibrium Morphology in Weakly Segregated Diblock Copolymers. Macromolecules 27, 4063 (1994).

[54] I. W. Hamley. The Physics of Block Copolymers. Oxford University Press, Oxford (1998).

[55] H. Hasegawa, T. Hashimoto, H. Kawai, T. P. Lodge, E. J. Amis, G. C. J., and C. C. HAN. SANS and SAXS studies on molecular conformation of a block polymer in microdomain space. Macromolecules 18, 67 (1985).

[56] T. Hashimoto, K. Nagatoshi, A. Todo, H. Hasegawa, and H. Kawai. Domain-boundary structure of styrene-isoprene block copolymer films cast from toluene solutions. Macromolecules 7(3), 364 (1974).

[57] E. Helfand. Theory of inhomogeneous polymers: Fundamentals of the Gaussian random-walk model. J. Chem. Phys. 62(3), 999 (1975).

[58] A. von Der Heydt, M. Müller, and A. Zippelius. Sequence Fractionation in Symmetric Random Block Copolymers. Macromolecules 43(7), 3161 (2010).

[59] A. VOn Der Heydt, M. Müller, and A. Zippelius. Three-phase coexistence with sequence partitioning in symmetric random block copolymers. Phys. Rev. E 83, 051131 (2011). 
[60] R. Holyst and M. Schick. Copolymers as amphiphiles in ternary mixtures: An analysis employing disorder, equimaxima, and Lifshitz lines. J. Chem. Phys. 96(10), 7728 (1992).

[61] R. M. Hornreich, M. Luban, and S. Shtrikman. Critical behavior at the onset of $\vec{k}$-space instability on the $\lambda$ line. Phys. Rev. Lett. 35(25), 1678 (1975).

[62] J. Houdayer and M. Müller. Deviations from the mean-field predictions for the phase behaviour of random copolymers melts. Europhys. Lett. 58(5), 660 (2002).

[63] J. Houdayer and M. Müller. Phase diagram of random copolymer melts: A computer simulation study. Macromolecules 37(11), 4283 (2004).

[64] J. Hubbard. Calculation of partition functions. Phys. Rev. Lett. 3, 77 (1959).

[65] M. L. Huggins. Solutions of long chain compounds. J. Chem. Phys. 9, 440 (1941).

[66] M. L. Huggins. Some properties of solutions of long-chain compounds. J. Phys. Chem. 46, 151 (1942).

[67] M. L. Huggins. Theory of solutions of high polymers. J. Am. Chem. Soc. 64, $1712(1942)$.

[68] M. L. Huggins. Thermodynamic properties of solutions of long-chain compounds. Ann. N. Y. Acad. Sci. 43, 1 (1942).

[69] P. K. Janert and M. Schick. Phase behavior of ternary homopolymer/diblock blends: Influence of relative chain lengths. Macromolecules 30, 137 (1997).

[70] P. K. Janert and M. Schick. Phase behavior of ternary homopolymer/diblock blends: Microphase unbinding in the symmetric system. Macromolecules 30(13), 3916 (1997).

[71] R. A. L. Jones. Soft Condensed Matter. Oxford University Press, New York (2002).

[72] A. K. Khandpur, S. Foerster, F. S. Bates, I. W. Hamley, A. J. Ryan, W. Bras, K. Almdal, and K. Mortensen. Polyisoprene-Polystyrene Diblock Copolymer Phase Diagram near the Order-Disorder Transition. Macromolecules 28(26), 8796 (1995).

[73] R. G. Kirste, W. A. Kruse, and K. Ibel. Determination of the conformation of polymers in the amorphous solid state and in concentrated solution by neutron diffraction. Polymer 16(2), 120 (1975).

[74] W. Kunn. Über die Gestalt fadenförmiger Moleküle in Lösungen. KolloidZeitschrift 68(1), 2 (1934).

[75] L. D. Landau. Zur Theorie der Phasenumwandlungen II. Phys. Z. Sow. 11, 26, 542 (1937). Reprinted in The Collected Papers of L. D. Landau, ed. D. ter Haar, Pergamon, New York (1965). 
[76] L. LEIBLER. Theory of microphase separation in block copolymers. Macromolecules 13, 1602 (1980).

[77] M. Levy. "Living Polymers"-50 years of evolution. Bull. Isr. Chem. Soc. 18 (2005).

[78] N. Madras and S. G. Whittington. Self-averaging in finite random copolymers. J. Phys. A: Math. Gen. 35, L427 (2002).

[79] M. W. Matsen. The standard Gaussian model for block copolymer melts. J. Phys.: Condens. Matter 14(2), R21 (2002).

[80] M. W. Matsen and F. S. Bates. Unifying Weak- and Strong-Segregation Block Copolymer Theories. Macromolecules 29, 1091 (1996).

[81] M. W. Matsen and M. Schick. Stable and unstable phases of a diblock copolymer melt. Phys. Rev. Lett. 72(16), 2660 (1994).

[82] A. D. McNaught and A. Wilkinson. IUPAC. Compendium of Chemical Terminology. Blackwell Scientific Publications, Oxford (1997).

[83] D. J. MeIER. Theory of block copolymers. I. Domain formation in A-B block copolymers. J. Polym. Sci.: Part C 26, 81 (1969).

[84] M. Mézard, G. Parisi, and M. A. Virasoro. Spin Glass Theory and Beyond, vol. 9 of Lecture Notes in Physics. World Scientific, Singapore (1987).

[85] F. M. Mirabella and E. ForD. Characterization of linear low-density polyethylene: Cross-fractionation according to copolymer composition and molecular weight. J. Polym. Sci.: Polym. Phys. 25, 777 (1987).

[86] F. M. Mirabella, S. P. Westphal, P. L. Fernando, E. A. Ford, and J. G. WiLliams. Morphological explanation of the extraordinary toughness of linear low density polyethylenes. J. Polym. Sci.: Polym. Phys. 26, 1995 (1988).

[87] D. Muckamel and R. M. HornReich. The nematic-smectic c phase transition: a renormalisation group analysis. J. Phys. C: Solid St. Phys. 13, 161 (1980).

[88] M. MÜLLER and M. SCHICK. Bulk and interfacial thermodynamics of a symmetric, ternary homopolymer-copolymer mixture: a Monte Carlo study. J. Chem. Phys. 105, 8885 (1996).

[89] M. Muthukumar. Fluctuation effects in the density functional theory of orderdisorder transitions in block copolymers. Macromolecules 26, 5259 (1993).

[90] J. R. Naughton and M. W. Matsen. Nonperiodic lamellar phase in ternary diblock copolymer/homopolymer blends. Macromolecules 35, 8926 (2002).

[91] A. Nesarikar, M. Olvera de la Cruz, and B. Crist. Phase transitions in random copolymers. J. Chem. Phys. 98(9), 7385 (1993). 
[92] H. Nishimori. Statistical Physics of Spin Glasses and Information Processing. Oxford University Press (2001).

[93] NIST. Copolymers block out new approaches to microelectronics. ScienceDaily (2008). URL http://www.sciencedaily.com/releases/2008/03/ $080312141238 . \mathrm{htm}$

[94] P. D. Olmsted. Lectures on Landau Theory of Phase Transitions (2000).

[95] I. I. Potemkin and S. V. PAnyukov. Microphase separation in correlated random copolymers: Mean-field theory and fluctuation corrections. Phys. Rev. E $\mathbf{5 7}(6), 6902$ (1998).

[96] O. S. Rabotyagova, P. Cebe, and D. L. Kaplan. Protein-Based Block Copolymers. Biomacromolecules 12, 269 (2011).

[97] E. Reister, M. Müller, and K. Binder. Spinodal decomposition in a binary polymer mixture: Dynamic self-consistent-field theory and Monte Carlo simulations. Phys. Rev. E 64, 041804 (2001).

[98] M. Rubinstein and R. H. Colby. Polymer Physics. Oxford University Press, Oxford (2003).

[99] L. Schäfer. Excluded Volume Effects in Polymer Solutions as explained by the renormalization group. Springer, Berlin (1999).

[100] K. S. Schweizer and J. G. Curro. Microscopic theory of the Structure, Thermodynamics, and Apparent $\chi$ Parameter of Polymer Blends. Phys. Rev. Lett. 60(9), 809 (1988).

[101] K. S. Schweizer and J. G. Curro. Integral equation theory of the structure and thermodynamics of polymer blends. J. Chem. Phys. 91(8), 5059 (1989).

[102] R. L. ScotT. The thermodynamics of high-polymer solutions: II. The solubility and fractionation of a polymer of heterogeneous distribution. J. Chem. Phys. 13(5), 178 (1945).

[103] R. L. ScotT. Thermodynamics of high polymer solutions. VI. The compatibility of copolymers. J. Polym. Sci. 9(5), 423 (1952).

[104] A. N. Semenov. Secondary super-structures in random copolymers. Eur. Phys. J. B 10, 497 (1999).

[105] J. P. Sethna. Statistical Mechanics: Entropy, Order Parameters, and Complexity, chap. 8.2. Oxford University Press (2006).

[106] C. D. Sfatos, A. M. Gutin, and E. I. Shakhnovich. Phase diagram of random copolymers. Phys. Rev. E 48(1), 465 (1993).

[107] C. D. Sfatos, A. M. Gutin, and E. I. Shakhnovich. Fluctuation effects of microphase separation in a random copolymer hamiltonian. J. Phys. A 27, L411 (1994). 
[108] E. I. Shakhnovich and A. M. Gutin. Formation of microdomains in a quenched disordered heteropolymer. J. Phys. (Paris) 50(14), 1843 (1989).

[109] S. W. Sides and G. H. Fredrickson. Parallel algorithm for numerical selfconsistent field theory simulations of block copolymer structure. Polymer 44, 5859 (2003).

[110] P. Sollich. Predicting phase equilibria in polydisperse systems. J. Phys.: Condens. Matter 14(3), R79 (2002).

[111] P. Sollich and N. B. Wilding. Crystalline Phases of Polydisperse Spheres. Phys. Rev. Lett. 104, 118302 (2010).

[112] C. E. Soteros and S. G. Whittington. The statistical mechanics of random copolymers. J. Phys. A: Math. Gen. 37, R279 (2004).

[113] J. Stejskal and P. Kratochvíl. Comparison of the instantaneous and conversion heterogeneity of random copolymers. J. Appl. Polym. Sci. 25, 407 (1980).

[114] W. H. Stockmayer. Distribution of chain lengths and composition in copolymers. J. Chem. Phys. 13(6), 199 (1945).

[115] M. P. Stoykovich, K. C. Daoulas, M. Müller, H. Kang, J. J. De Pablo, and P. F. NeAley. Remediation of line edge roughness in chemical nanopatterns by the directed assembly of overlying block copolymer films. Macromolecules 43, 2334 (2010).

[116] R. L. Stratonovich. On a method of calculating quantum distribution functions. Doklady Akad. Nauk SSSR [transl.: Sov. Phys. Dokl.] 115 [2], 1097 [416] (1957).

[117] G. Strobl. The Physics of Polymers. Springer, Berlin, 3rd edn. (2007).

[118] A. V. Subbotin and A. N. Semenov. Phase equilibria in random block copolymer melts. Eur. Phys. J. E 7, 49 (2002).

[119] J. SwifT and P. C. Hohenberg. Hydrodynamic fluctuations at the convective instability. Phys. Rev. A 15(1), 319 (1977).

[120] J. SwifT and P. LeITNER. Models for phase transitions with continuous constant energy surfaces. Phys. Rev. B 16, 4137 (1977).

[121] M. Szwarc, M. Levy, and R. Milkovich. Polymerization initiated by electron transfer to monomer. A new method of formation of block polymers. J. Am. Chem. Soc. 78, 2656 (1956).

[122] E. L. Thomas, D. B. Alward, D. J. Kinning, and D. C. Martin. Ordered Bicontinuous Double-Diamond Structure of Star Block Copolymers: A New Microdomain Morphology. Macromolecules 19, 2197 (1986).

[123] N. Torikai, Y. Matsushita, and T. Ebisawa. Microphase-separated interface of a two-component triblock copolymer with a lamellar structure. Physica B 248, 284 (1998). 
[124] K. Šolc (ed.). Polymer Compatibility and Incompatibility: Principles and Practices. Harwood Academic, Chur (1982).

[125] C. WALD. Phase behavior of random copolymers and crosslinked homopolymer melts. Ph.D. thesis, Universität Göttingen (2005).

[126] A. M. Welander, H. Kang, K. O. Stuen, H. H. Solak, M. Müller, J. J. DE PABlo, and P. F. NeAley. Rapid directed assembly of block copolymer films at elevated temperatures. Macromolecules 41, 2759 (2008). 



\section{Danksagungen}

Es gibt viele Menschen, ohne deren Unterstützung und wohlwollende Begleitung es diese Arbeit nicht gäbe, und denen ich hier meinen aufrichtigen Dank aussprechen möchte.

Zuerst möchte ich Prof. Dr. Annette Zippelius danken, die mir ermöglicht hat, dieses interessante Thema zu bearbeiten, mich in all den Jahren motiviert betreut und mir dabei äußerst viel Freiraum eingeräumt hat. Prof. Dr. Marcus Müller danke ich für interessante Diskussionen und Anregungen, und für prompte Antworten per email während der Arbeit zu Fraktionierung. Besonderen Dank schulde ich Prof. Dr. Peter Müller für die Übernahme des Korreferats.

Den guten Geistern des Instituts, besonders Katrin Glormann, Bärbel Lütge-Hampe, Gisela Mesecke und Gabriele Schubert, danke ich für ihre Freundlichkeit, Hilfsbereitschaft und unbürokratische Unterstützung in allen Situationen. Dies gilt ebenso für Michaela Liebmann vom SFB 602 und Rebecca Benez vom SFB 937.

Unzählige anregende Gespräche, ausgedehnte Tee- oder Kaffee-Runden und sogar gelegentlichen Kulturgenuss verdanke ich Stephan, Martin, Hildegard, Robert, (Opa) Riccardo, Till, Timo Aspelmeier, Timo Fischer, Jonathan sowie vielen Bewohnern der 3./4. Etage, die sich hier erwähnt fühlen mögen. Auch Treffen mit den „Ehemaligen“ Hendrik und Katharina, meist bei der DPG-Tagung, waren inspirierend.

Ohne die langjährige, aufmerksame Physiotherapie-Begleitung durch Irene Fährmann wäre die Arbeit sicherlich auch unmöglich gewesen.

Danken möchte ich meinen Eltern und meiner Schwester, die diese Arbeit mit Interesse begleitet haben und auf die ich mich stets verlassen konnte, nicht nur beim letzten Korrekturlesen. Nicht zuletzt für viele Stunden Zuhören, Geduld und großes Einfühlungsvermögen danke ich Till, der wissen wird, wofür alles noch. 



\section{Lebenslauf}

\section{Persönliche Daten}

Name:

Geburtstag und -ort:

Adresse:

Nationalität:

\section{Schulbildung}

1984-1988

1988-1990

1990-1997

1994-1995

Juni 1997

1997-1998

\section{Studium}

Oktober 1998

Juli 2001

Januar 2005

September 2005

\section{Publikationen}

Alice von der Heydt

17.12.1977 in Regensburg

Am Pfingstanger 52, 37075 Göttingen

deutsch

Hölty-Grundschule Göttingen

Orientierungsstufe Göttingen-Nord

Max-Planck-Gymnasium Göttingen

Colegio Sto. Thomas Morus, Santiago de Chile

Allgemeine Hochschulreife, Note: 1,0

Freiwilliges Soziales Jahr

Beginn Studium Physik an der Universität Göttingen

Vordiplom Physik, Note: sehr gut

Diplom Physik, Note: sehr gut, bei Prof. Dr. Annette Zippelius, Institut für Theoretische Physik, Universität Göttingen;

Diplomarbeit: Relaxationsdynamik von gelierenden Polymeren: Spannungs-Dehnungs-Relationen und dielektrische Suszeptibilität (2004)

Beginn Promotion Physik bei Prof. Dr. Annette Zippelius über Nichtlineare Elastizität von Polymernetzwerken, Institut für Theoretische Physik, Universität Göttingen;

Beginn Projekt über Heterogene Block-Copolymere Juli 2007

A. von der Heydt, M. Müller, and A. Zippelius. Sequence Fractionation in Symmetric Random Block Copolymers. Macromolecules 43(7), 3161 (2010).

A. von der Heydt, M. MÜller, and A. Zippelius. Threephase coexistence with sequence partitioning in symmetric random block copolymers. Phys. Rev. E 83, 051131 (2011). 